\title{
Synthesis of a-Substituted Enoximes with Nucleophiles via Nitrosoallenes
}

Hiroki Tanimoto, ${ }^{*}$ Keiichi Yokoyama, Yusuke Mizutani, Takashi Shitaoka, Tsumoru Morimoto, Yasuhiro Nishiyama, Kiyomi Kakiuchi

Graduate School of Materials Science, Nara Institute of Science and Technology (NAIST), 8916-5 Takayamacho, Ikoma, Nara 630-0192, Japan

$$
\text { tanimoto@ms.naist.jp }
$$

\section{Contents}

Tables of substrate preparation

Pictures of reaction mixture of nitrosoallene

Low temperature IR spectra of reaction mixtures 


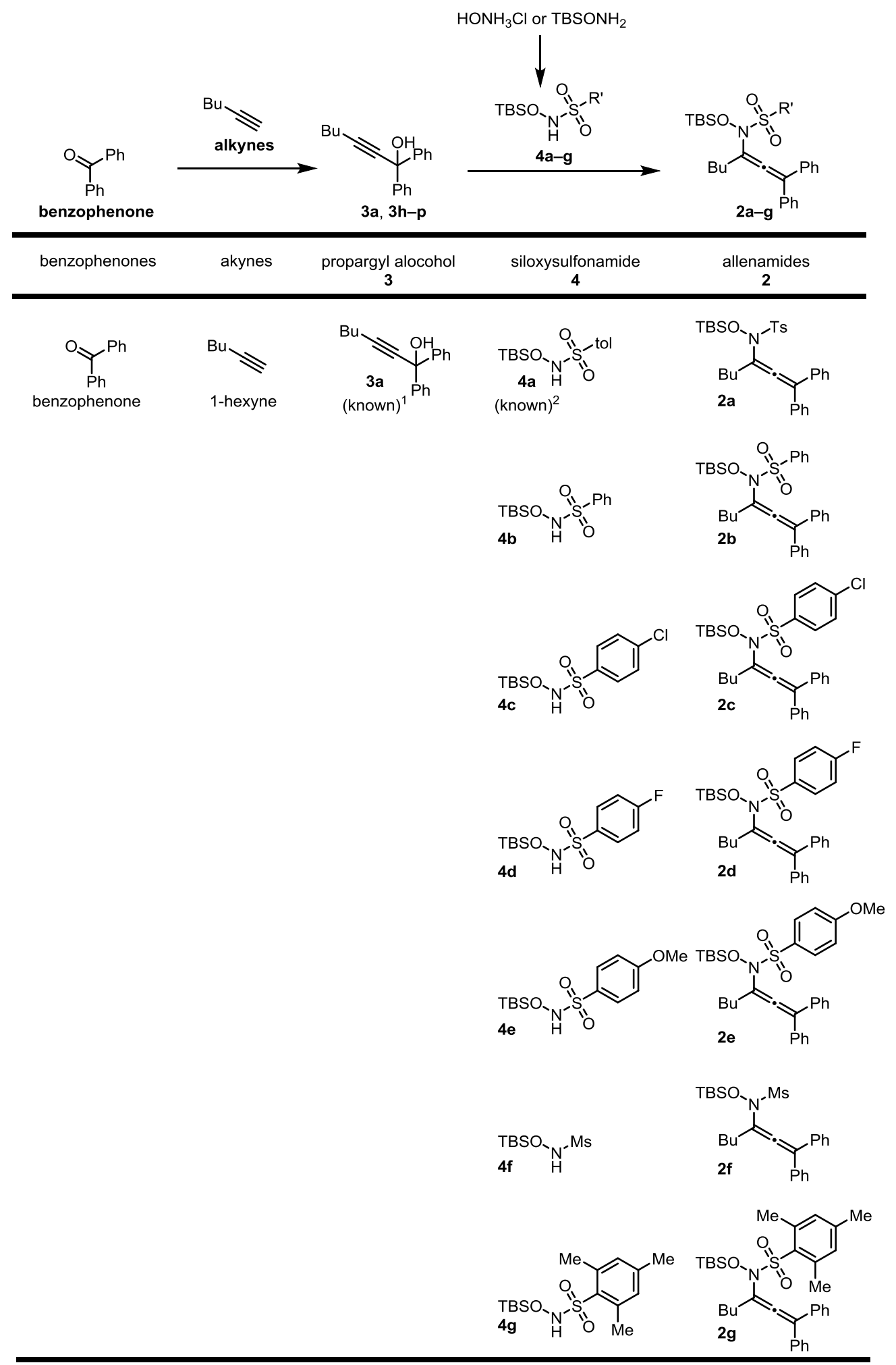

\section{References}

(1) (a) Zhang, H.; Tanimoto, H.; Morimoto, T.; Nishiyama, Y.; Kakiuchi, K. Org. Lett. 2013, 15, 5222. (b) Zhang, H.; Tanimoto, H.; Morimoto, T.; Nishiyama, Y.; Kakiuchi, K. Tetrahedron 2014, 70, 9828.

(2) (a) Kitahara, K.; Toma, T.; Shimokawa, J.; Fukuyama, T. Org. Lett. 2008, 10, 2259. (b) Kitahara, K.; Toma, T.; Shimokawa, J.; Fukuyama, T. Org. Synth. 2012, 89, 480. 


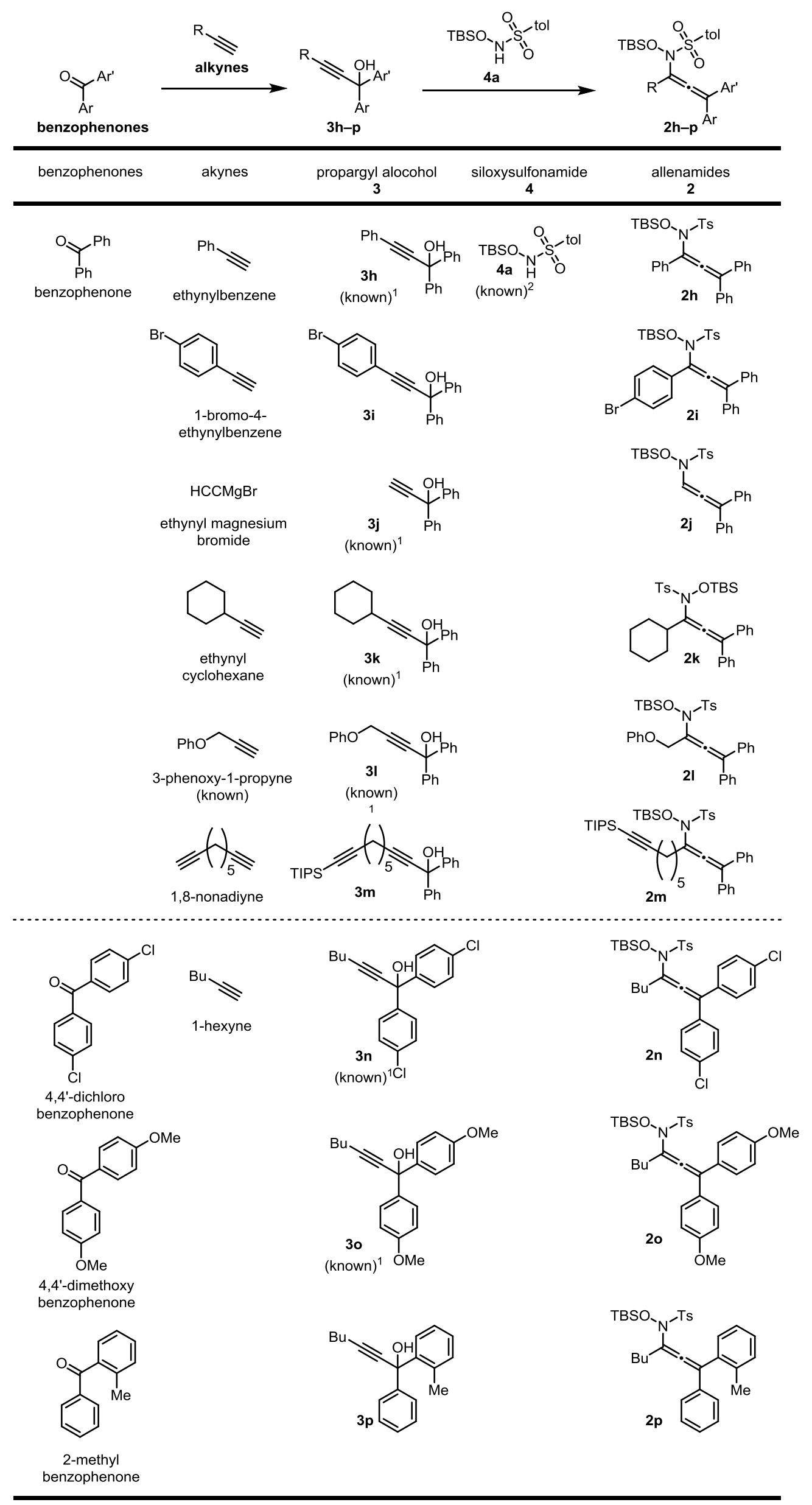




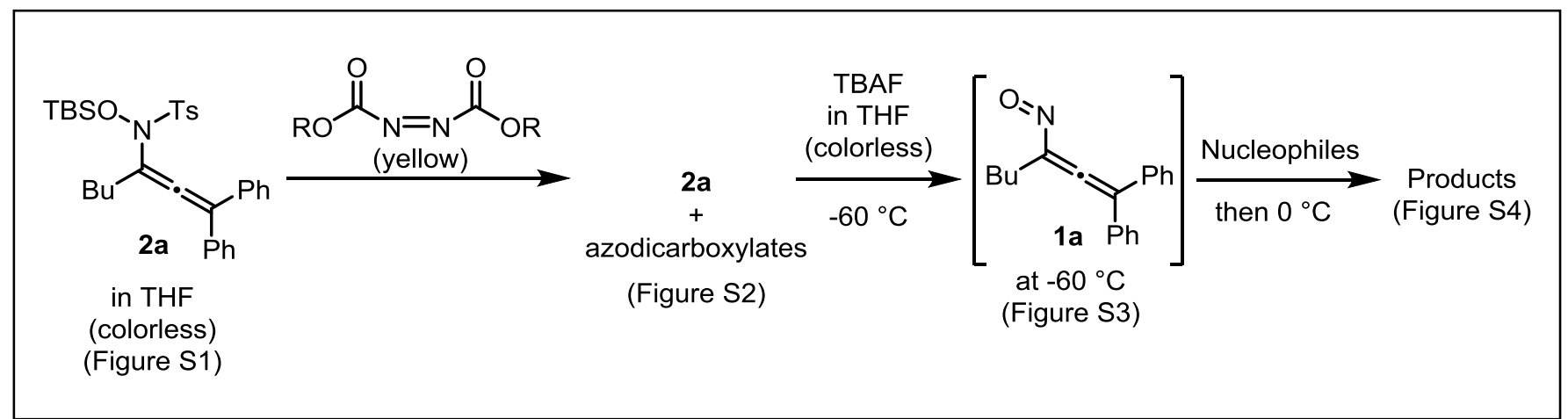

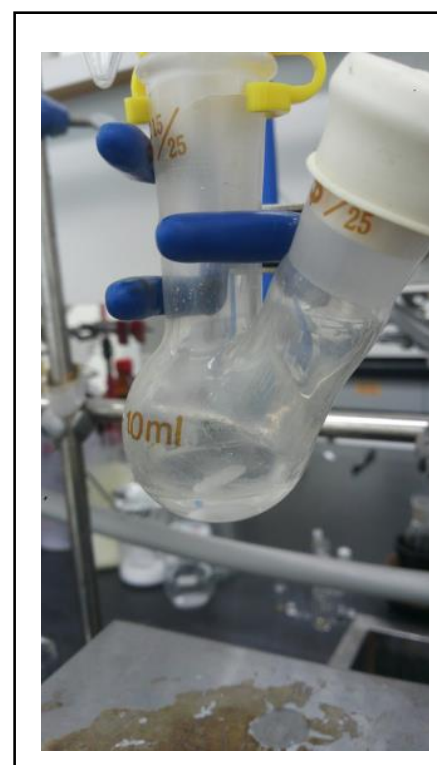

Figure S1
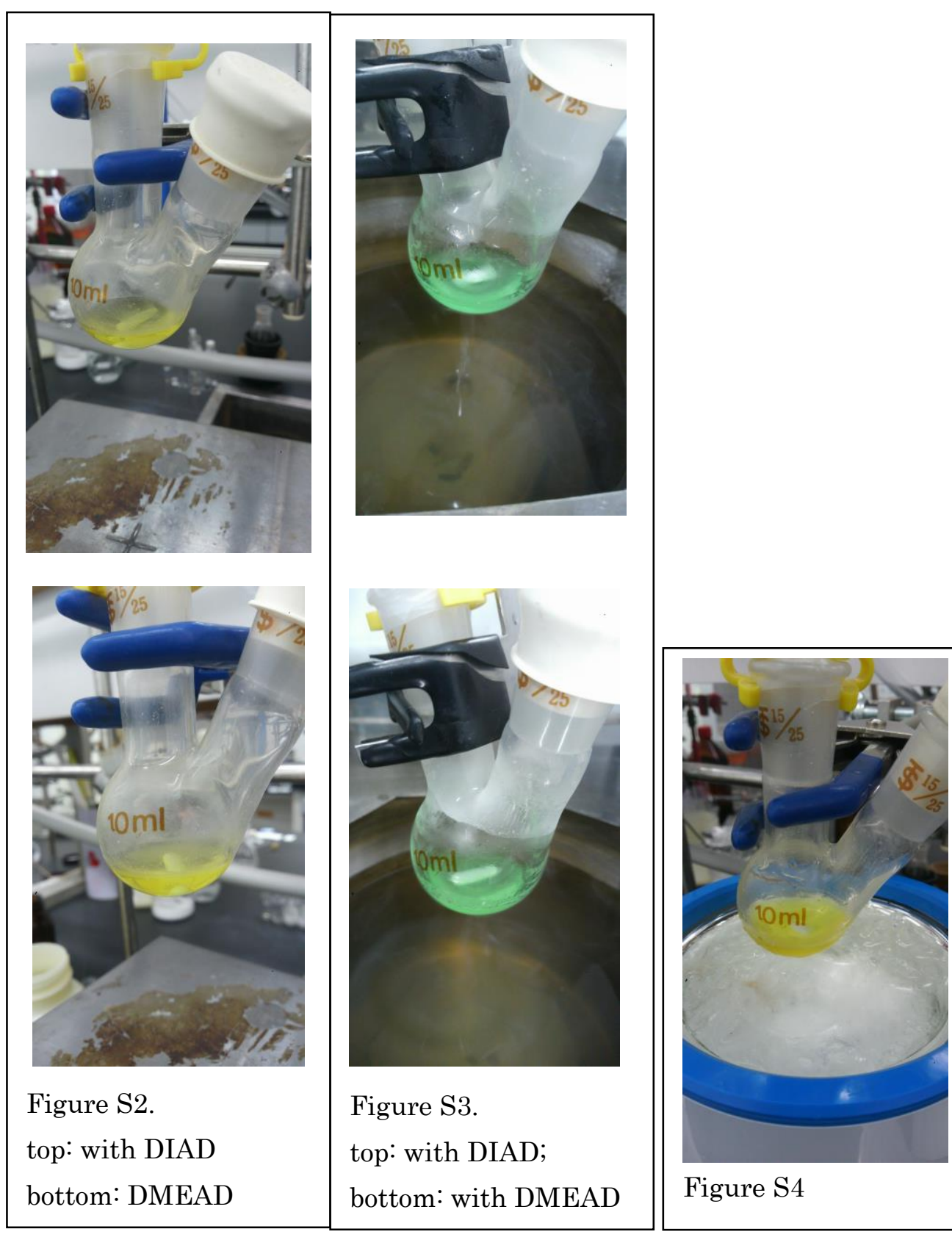

Figure $\mathbf{S} 4$ 
(2) mixture before TBAF addition at $-60^{\circ} \mathrm{C}$ (yellow)

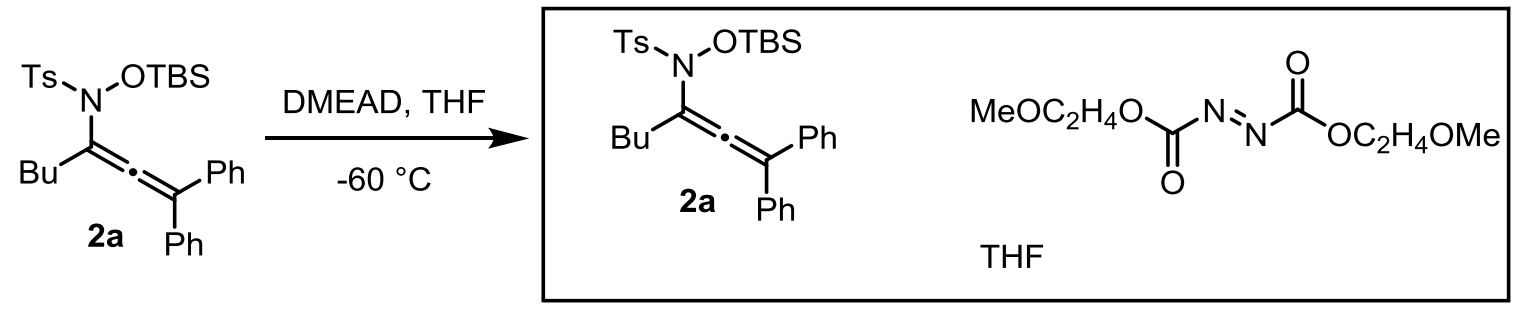

(3) mixture after TBAF addition at $-60^{\circ} \mathrm{C}$ (peppermint green)

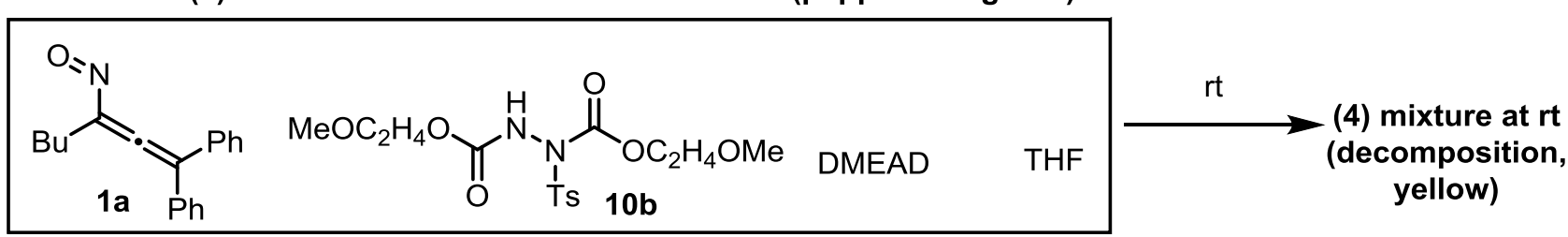

(1) THF at $-60^{\circ} \mathrm{C}$

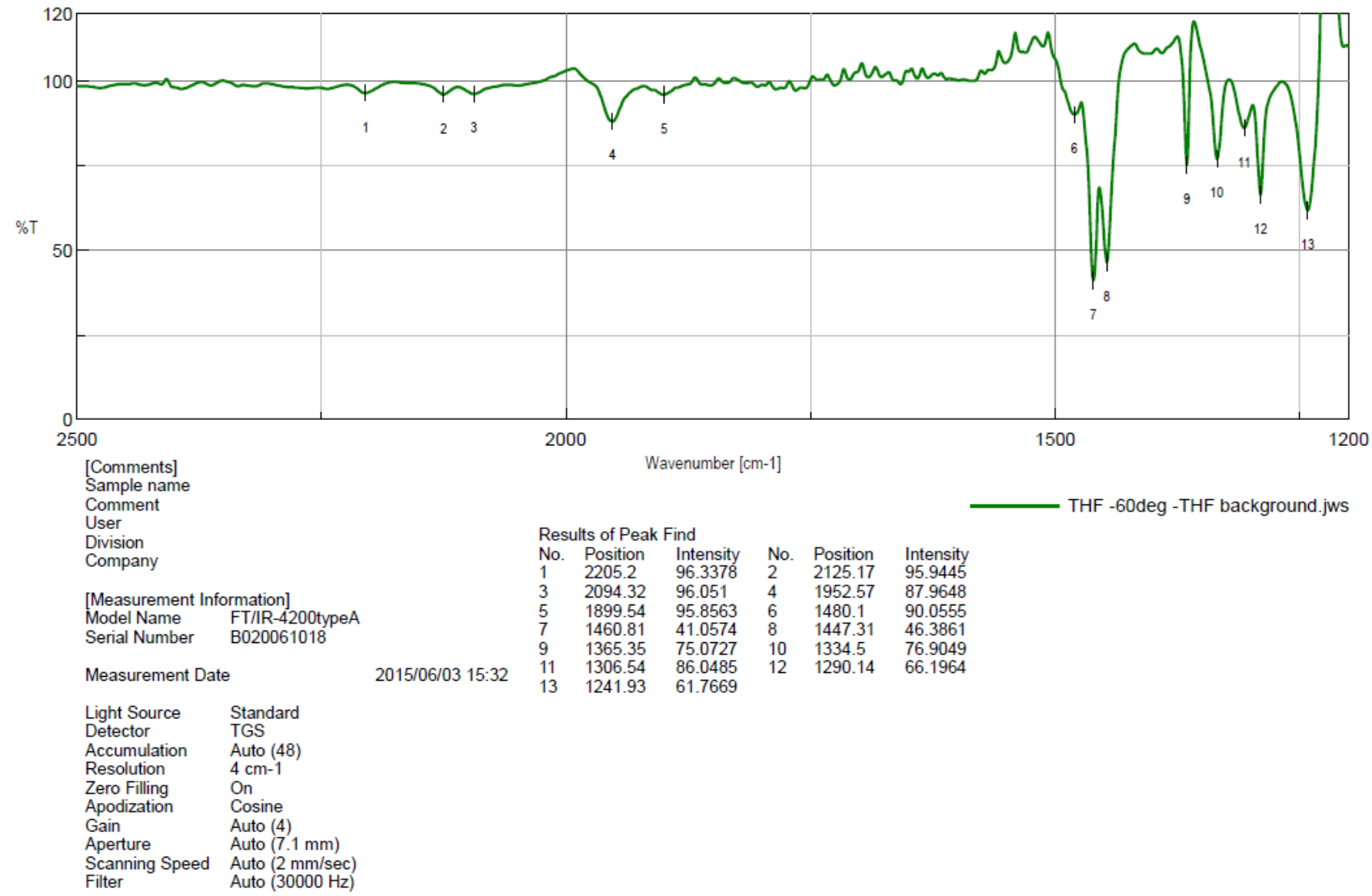


(2) Mixture before TBAF addition at $-60^{\circ} \mathrm{C}$ (Yellow solution)

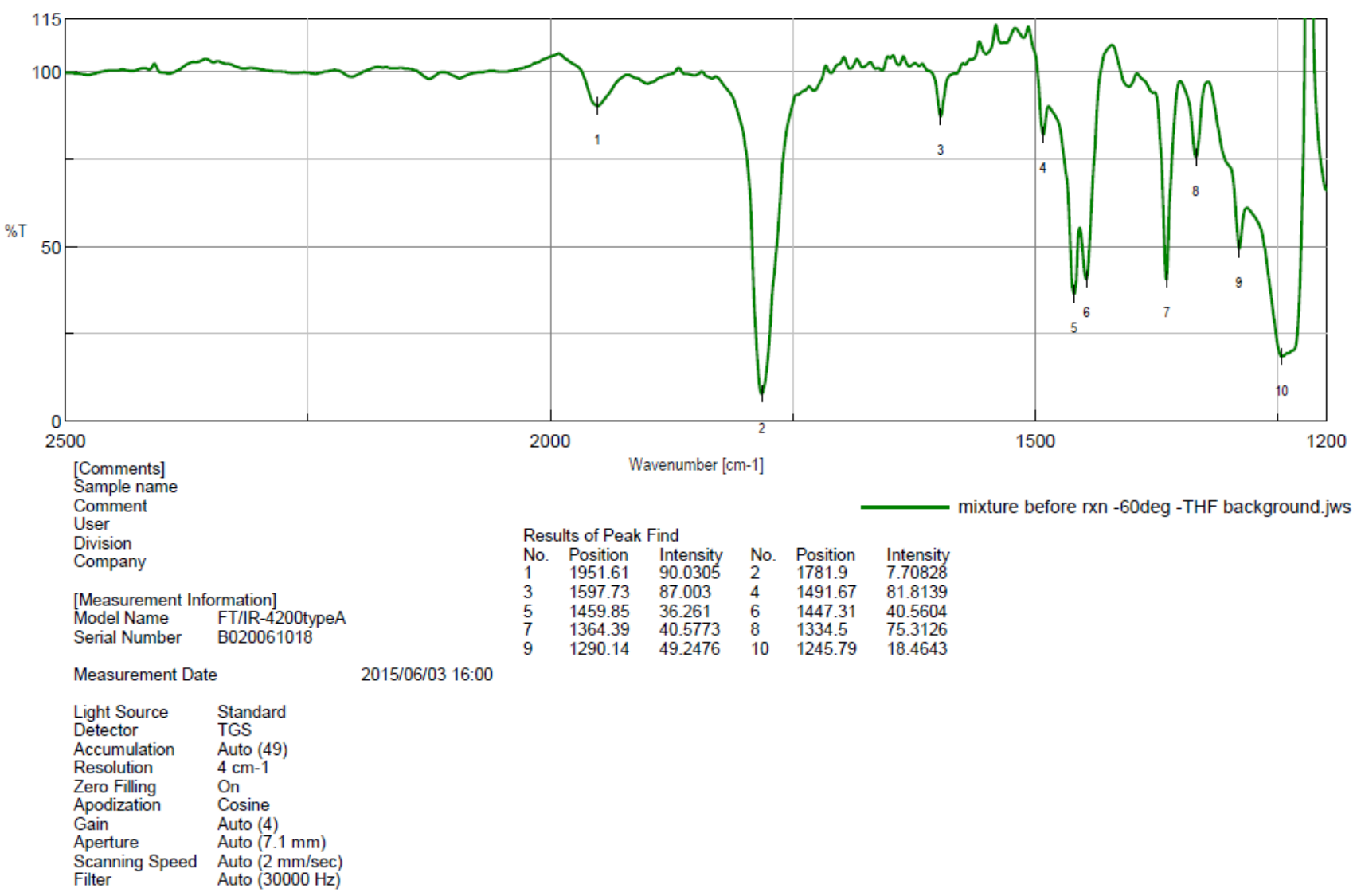

-Differential Spectrum (Subtracted THF at $-60^{\circ} \mathrm{C}$ )

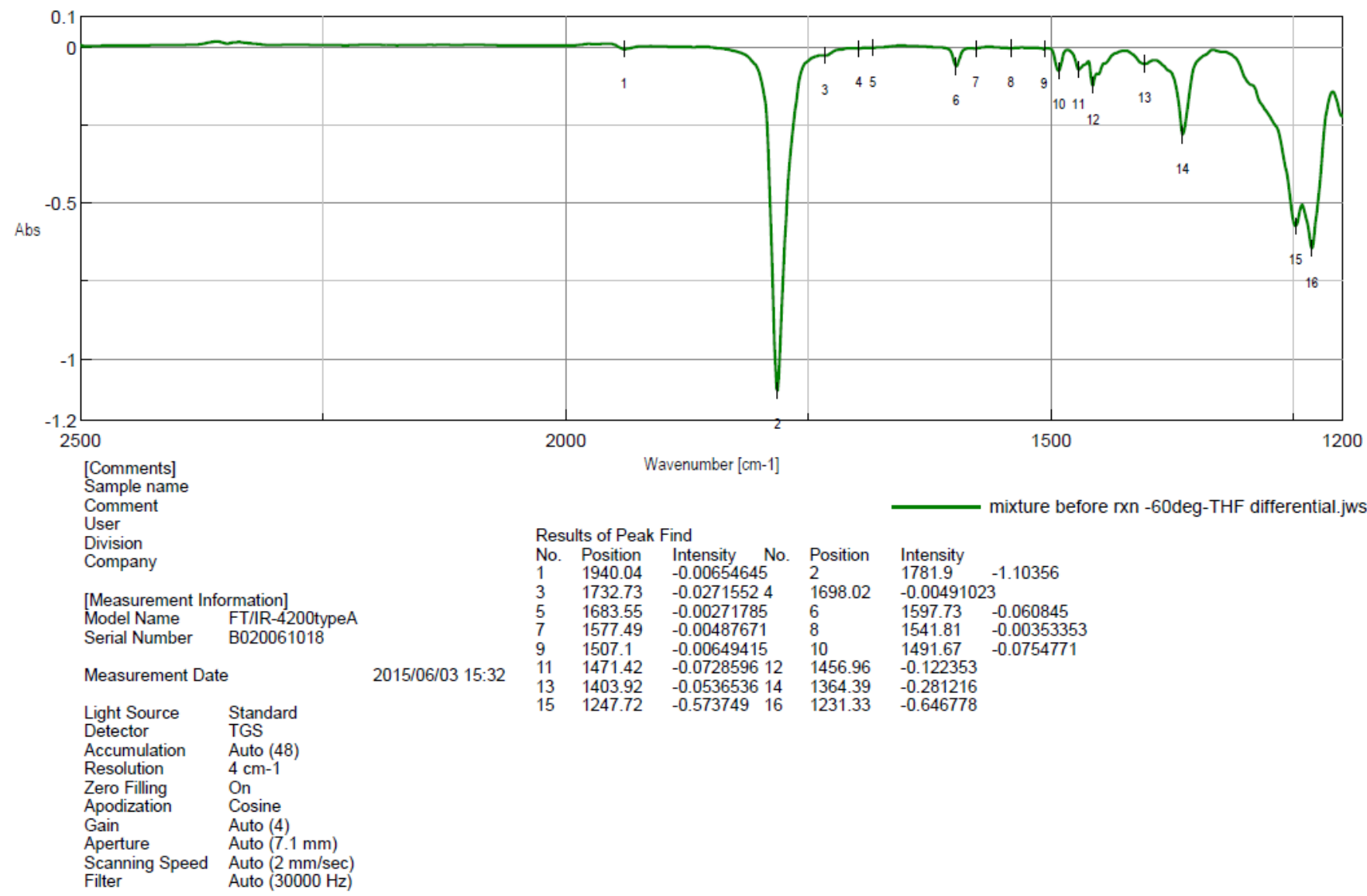


(3) Mixture after TBAF addition at $-60^{\circ} \mathrm{C}$ (peppermint green solution)

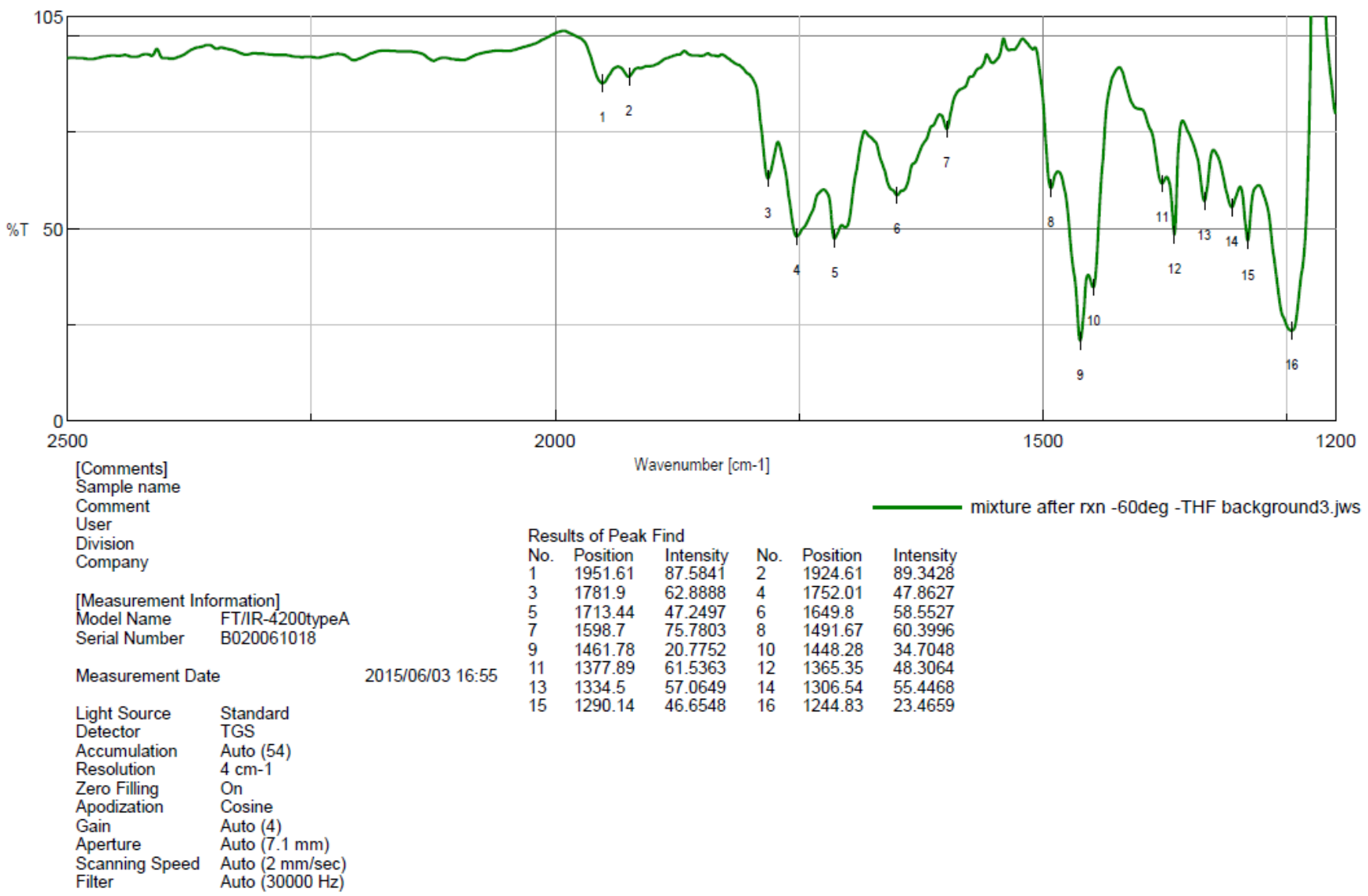

-Differential Spectrum (Subtracted THF at $-60^{\circ} \mathrm{C}$ )

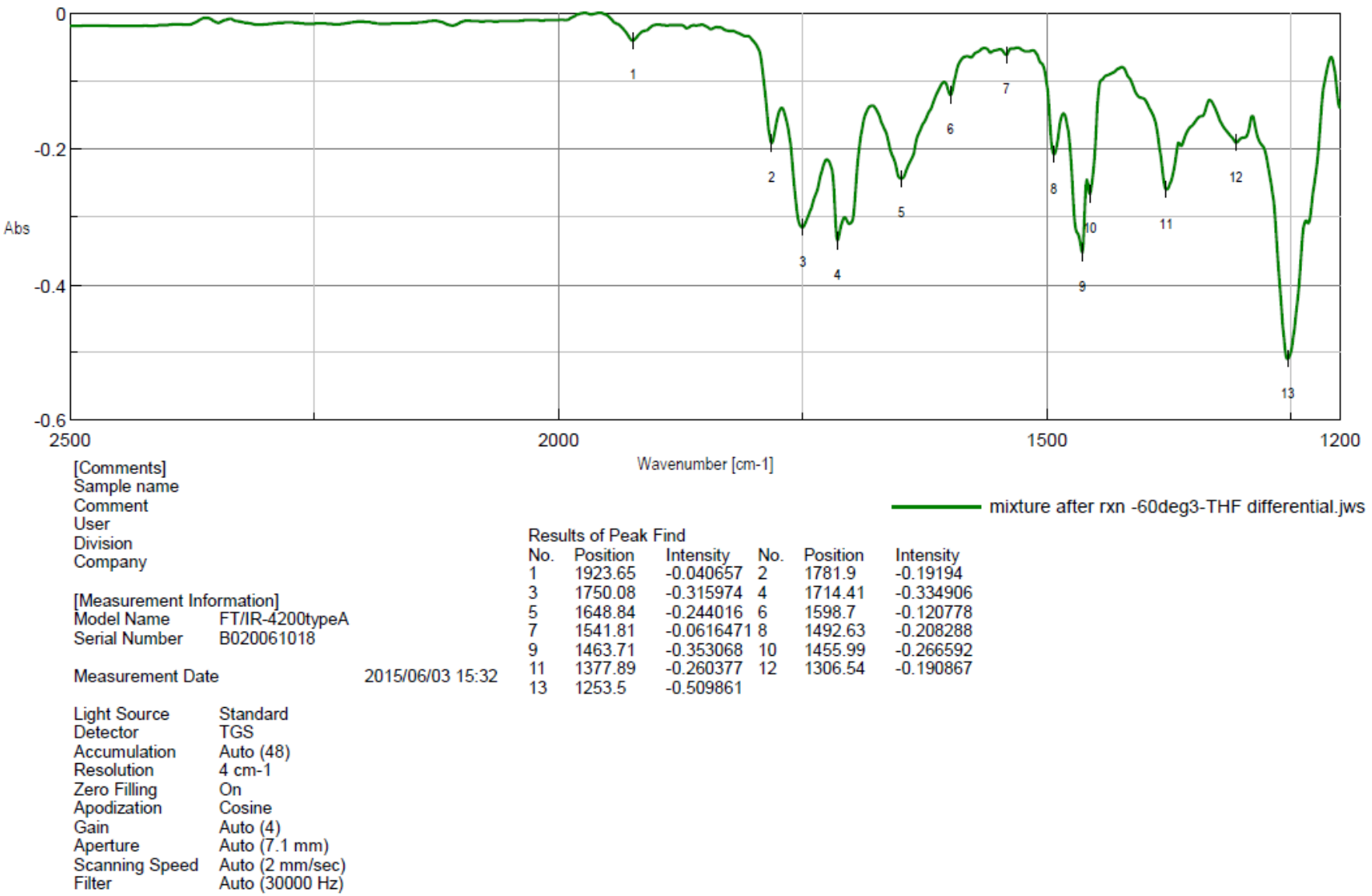


(4) Mixture at rt (yellow solution)

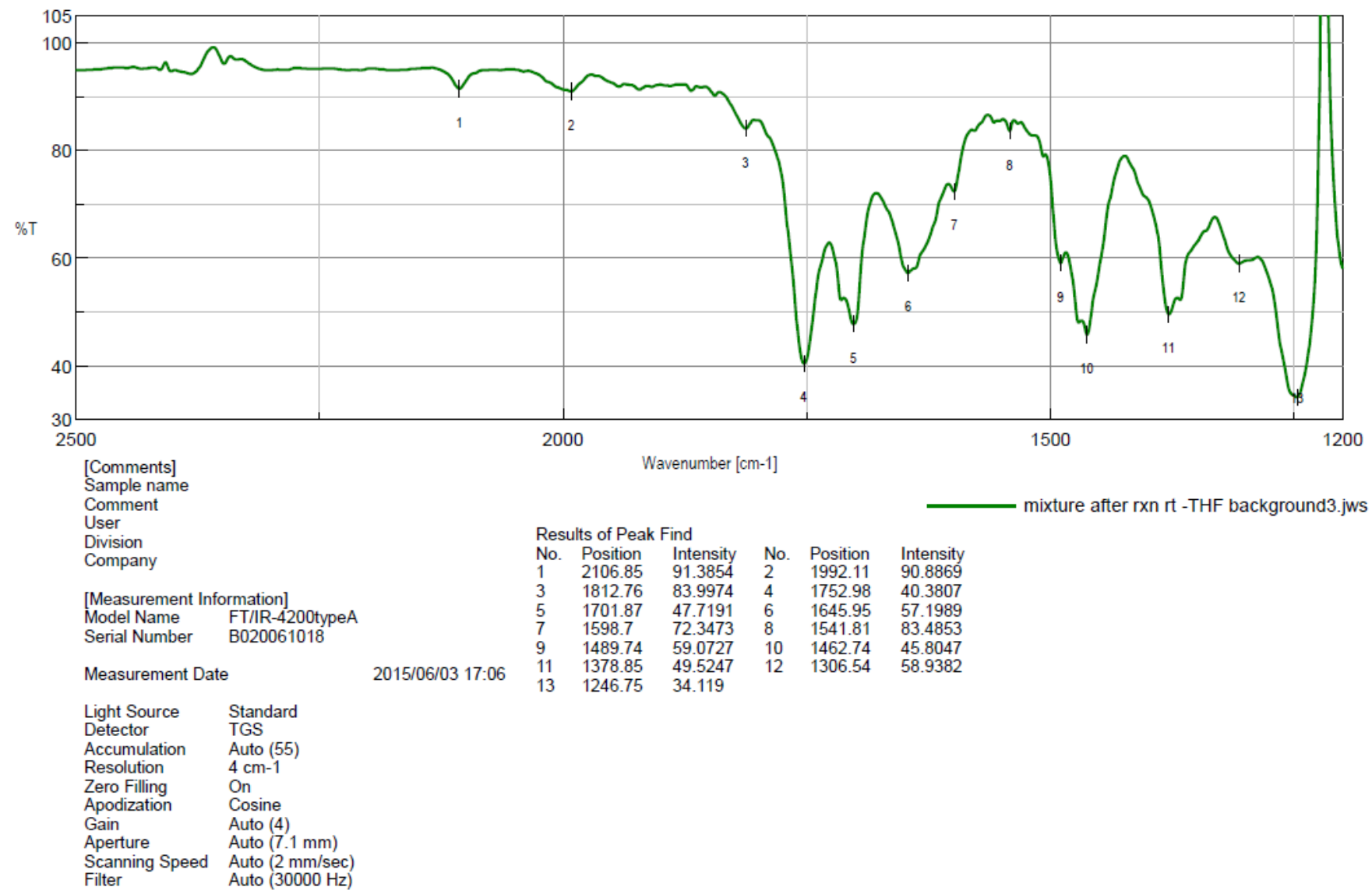

-Differential Spectrum (Subtracted THF at rt)

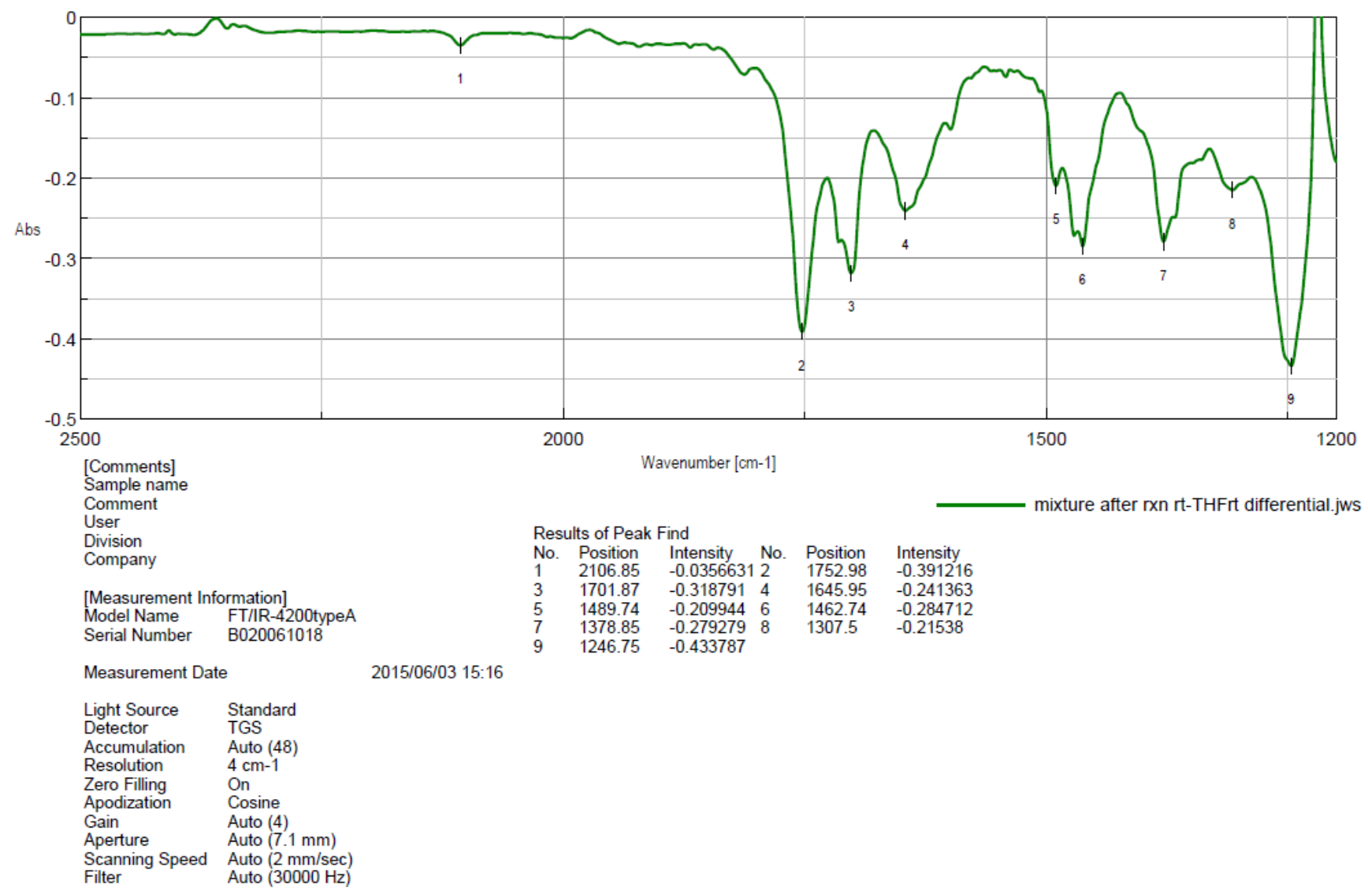




\section{Note}

- THF absorption peaks (3000-1200 cm-1): 3000-2800, 1953, 1480, 1461, 1447, 1365, $1335 \mathrm{~cm}^{-1}$

- Absorption peaks in (2): 1952 (THF), 1940 (allene of 2a, see defferential spectrum) 1782 (DMEAD), 1598 (sulfonamide in $2 \mathrm{a}$ ) $\mathrm{cm}^{-1}$

- New absorption peak around $2000 \mathrm{~cm}^{-1}$ in (3): $1924 \mathrm{~cm}^{-1}$.

This peak was disappeared after warming up to room temperature (4) as indicated by solution color.

- Reactions in dichloromethane, chloroform or carbon tetrachloride did not proceed at low temperature. 

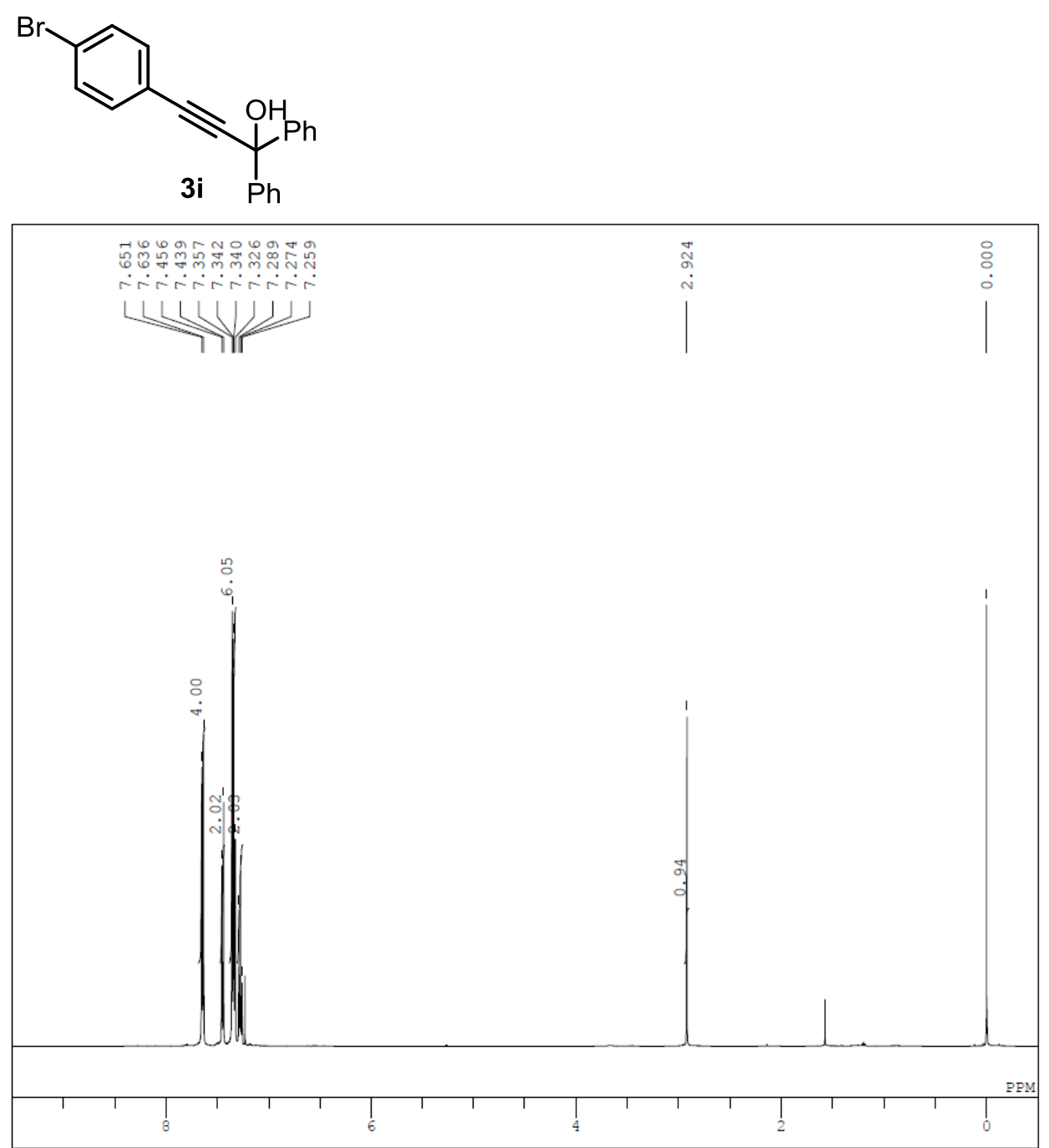

DFILE $\quad{ }_{\text {COMNT }}^{\text {Ym }}-05-124-1-1-1 . j \mathrm{df}$

DATIM 2013-12-27 16:34:34

OBNUC 1 H

EXMOD proton.jxp

$\begin{array}{lr}\text { OBFRQ } & 500.16 \mathrm{MHz} \\ \text { OBSET } & 2.41 \mathrm{KHz}\end{array}$

OBFIN $\quad 6.01 \mathrm{~Hz}$

POINT 16304

FREQU $9384.38 \mathrm{~Hz}$

$\begin{array}{lr}\text { SCANS } & 8 \\ \text { ACQTM } & 1.7459 \mathrm{sec}\end{array}$

$\begin{array}{ll}\text { ACQTM } & 1.7459 \mathrm{sec} \\ \mathrm{PD} & 5.0000 \mathrm{sec}\end{array}$

EN1 1 . 6.22 usec

IRNUC $1 \mathrm{H}$

SLVNT CDCL3

$\begin{array}{ll}\text { EXREF } & 0.00 \mathrm{ppm} \\ \text { BF } & 0.10 \mathrm{~Hz}\end{array}$

$\begin{array}{ll}\text { BE } & 0.10 \mathrm{H}\end{array}$

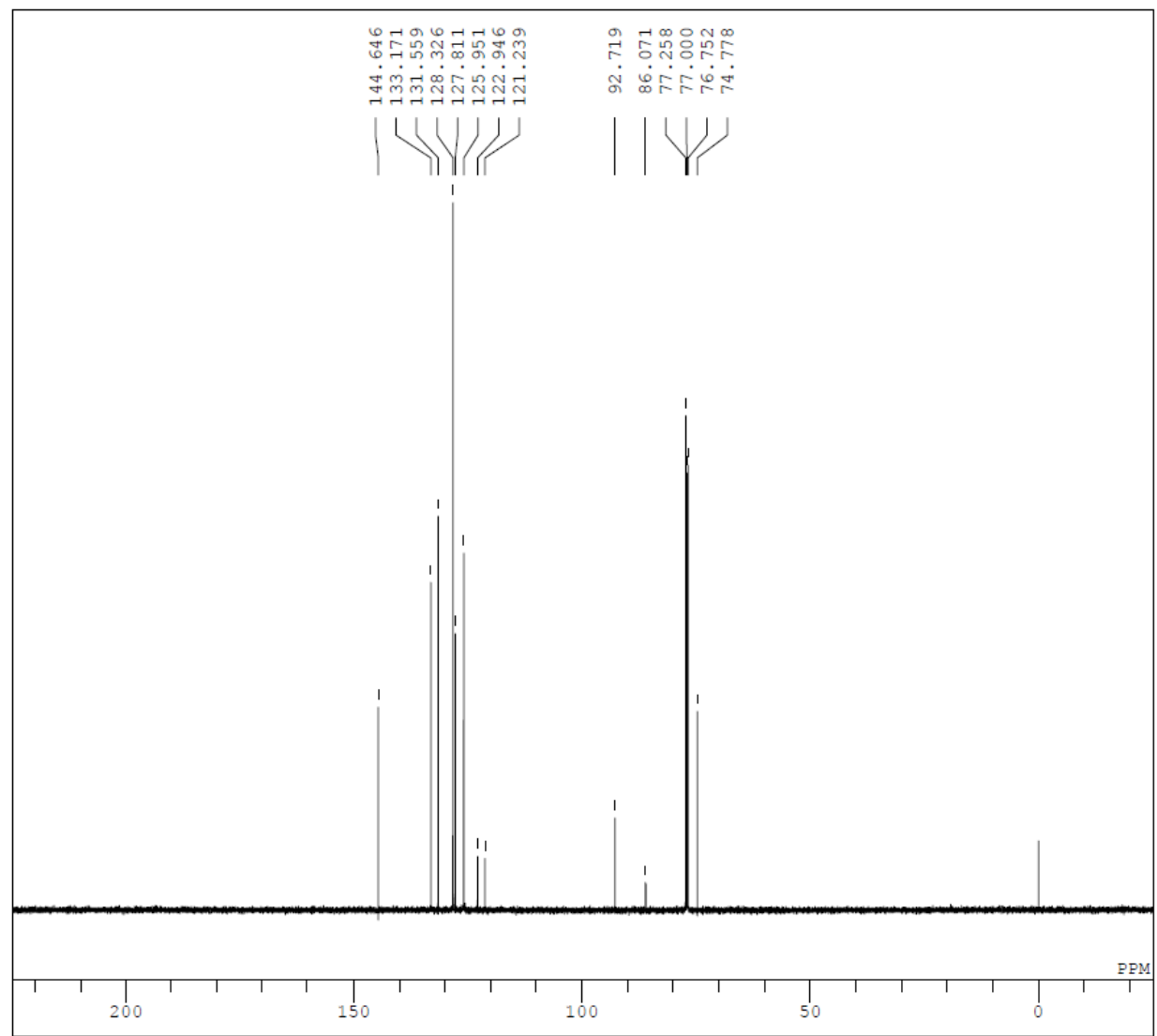

DFILE Y Ym_05_124_1_13C-1-1.jdf

DATIM 2013-12-27 16:36:10

OBNUC $13 \mathrm{C}$

EXMOD carbon.jXp

OBFRQ $\quad 125.77 \mathrm{MHz}$

OBFIN $4.21 \mathrm{~Hz}$ POINT 32767

FREQU $39308.18 \mathrm{~Hz}$

SCANS $\quad 512$

ACQTM $\quad 0.8336$ sec

$\begin{array}{lr}\text { PD } & 2.0000 \text { sec } \\ \text { PW1 } & 3.12 \text { usec }\end{array}$

IRNUC $1 \mathrm{H}$

CTEMP SLVNT CDCL 3

EXREF $\quad 77.00 \mathrm{pp}$

$\begin{array}{lr}\text { BF } & 0.10 \mathrm{~Hz} \\ \text { RGATN } & 56\end{array}$ 

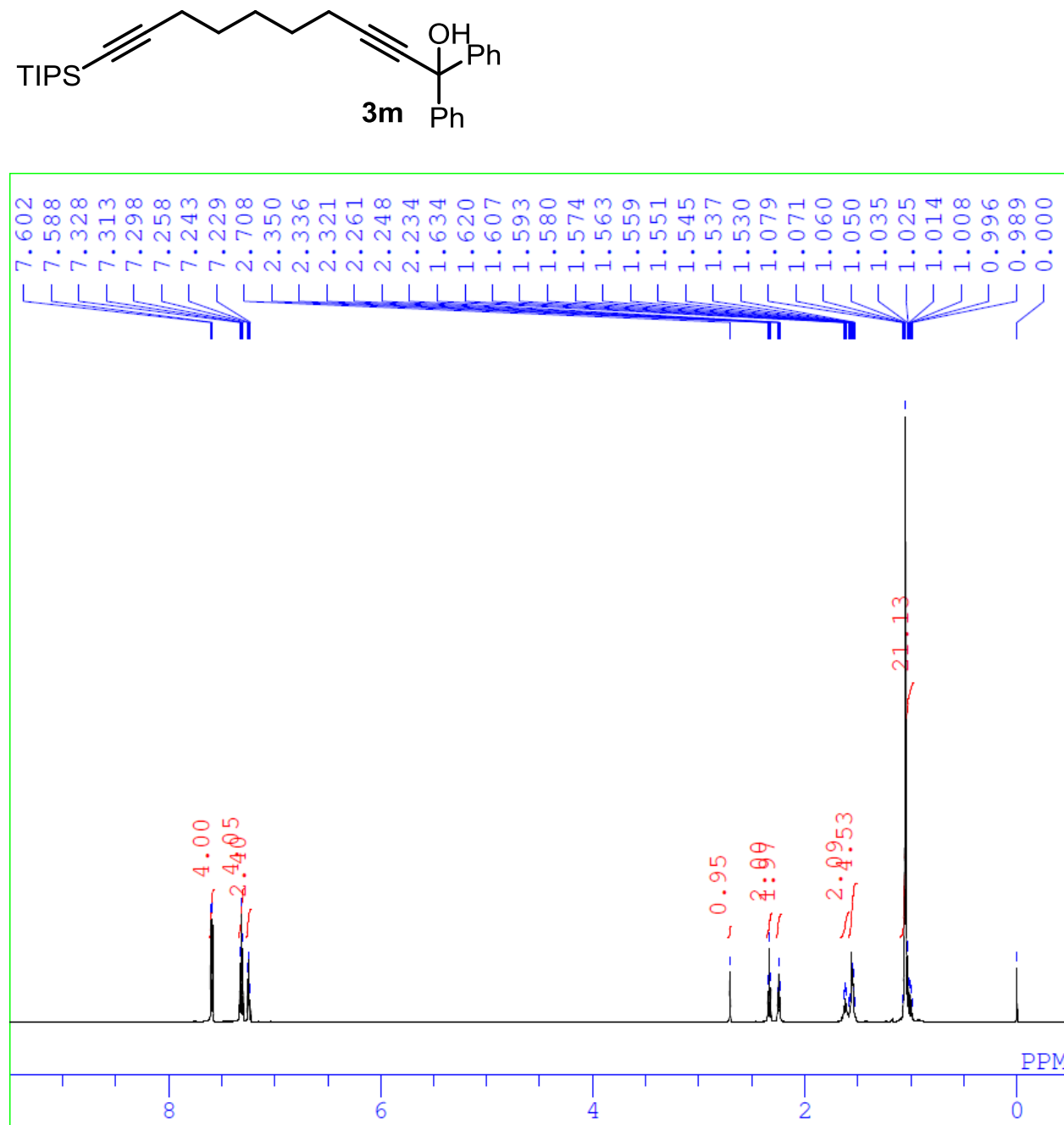

DFILE

COMNT

DATIM

OBNUC

EXMOD

OBFRQ

OBSET

OBFIN

POINT

FREQU

SCANS

ACQTM

$\mathrm{PD}$

PW1

IRNUC

CTEMP

SLVNT

EXREF

$\mathrm{BE}$

RGAIN ym 06015 1-1-1.al single_pulse

2014-09-02 14:51:53 $1 \mathrm{H}$

proton.jxp

$500.16 \mathrm{MHz}$

2. $41 \mathrm{KHz}$

$6.01 \mathrm{~Hz}$

13107

$7507.51 \mathrm{~Hz}$

1. $7459 \mathrm{sec}$

$5.0000 \mathrm{sec}$

$1 \mathrm{H}$

6.22 usec

CDCL 3

$17.5 \mathrm{c}$

$0.00 \mathrm{ppm}$

$0.10 \mathrm{~Hz}$

30

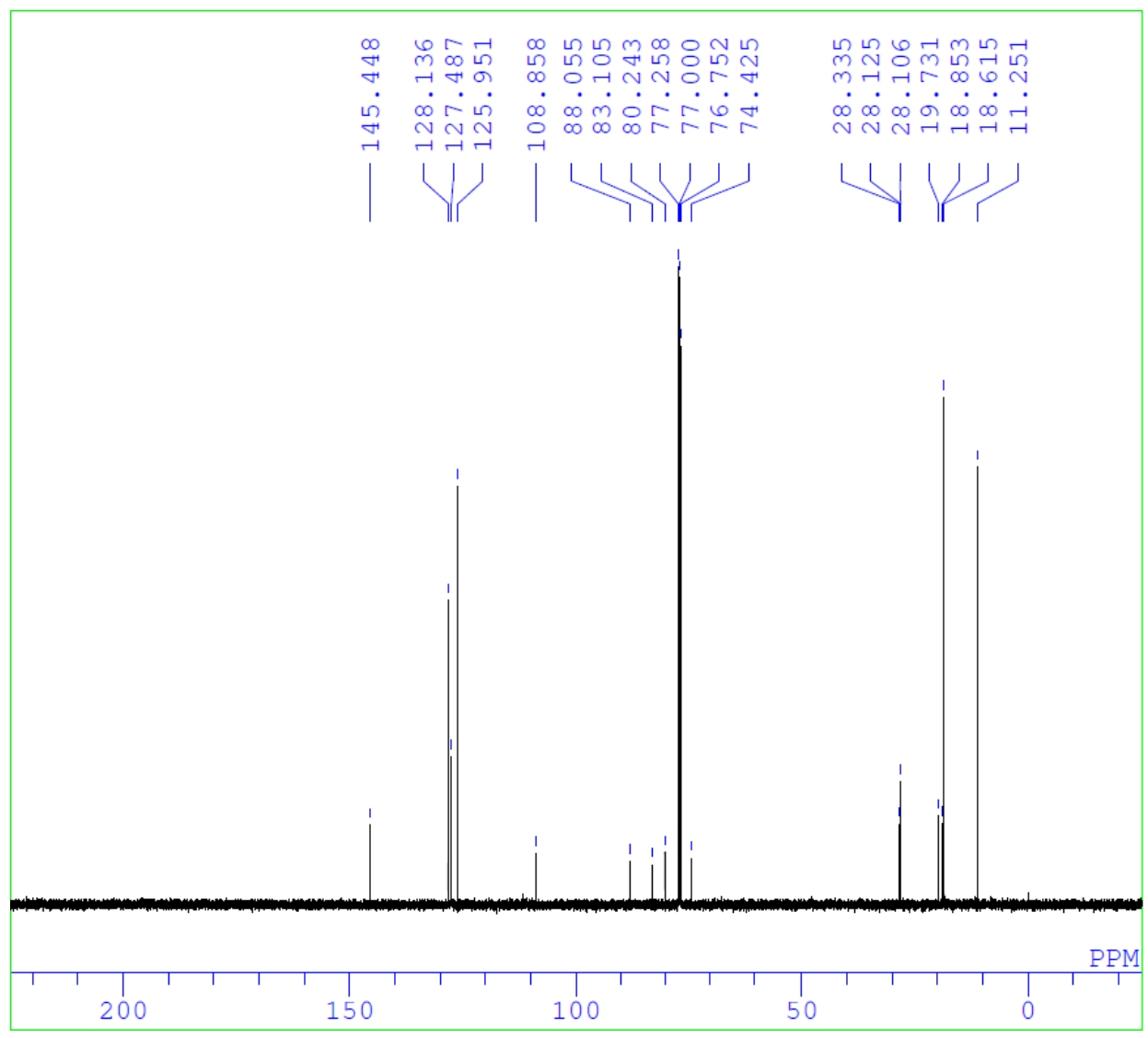

DEILE

COMNT

DATIM

OBNUC

EXMOD

OBERO

OBSET

OBE IN

POINT

FREQU

SCANS

ACQTM

$\mathrm{PD}$

PW1

IRNUC

CTEMP

SLVNT

EXREF

$\mathrm{BE}$

RGAIN ym $06 \quad 015 \quad 1 \quad 13 \mathrm{C}-1$ single pulse decoup 2014-09-03 01:51:12 $13 \mathrm{C}$

carbon.jxp

$125.77 \mathrm{MHz}$

$7.87 \mathrm{KHz}$

$4.21 \mathrm{~Hz}$

26214

$31446.54 \mathrm{~Hz}$

512

0.8336 sec

2.0000 sec

$1 \mathrm{H}$

3.12 usec

CDCL 3

$20.1 \mathrm{C}$

$77.00 \mathrm{ppm}$

$0.10 \mathrm{~Hz}$

56 

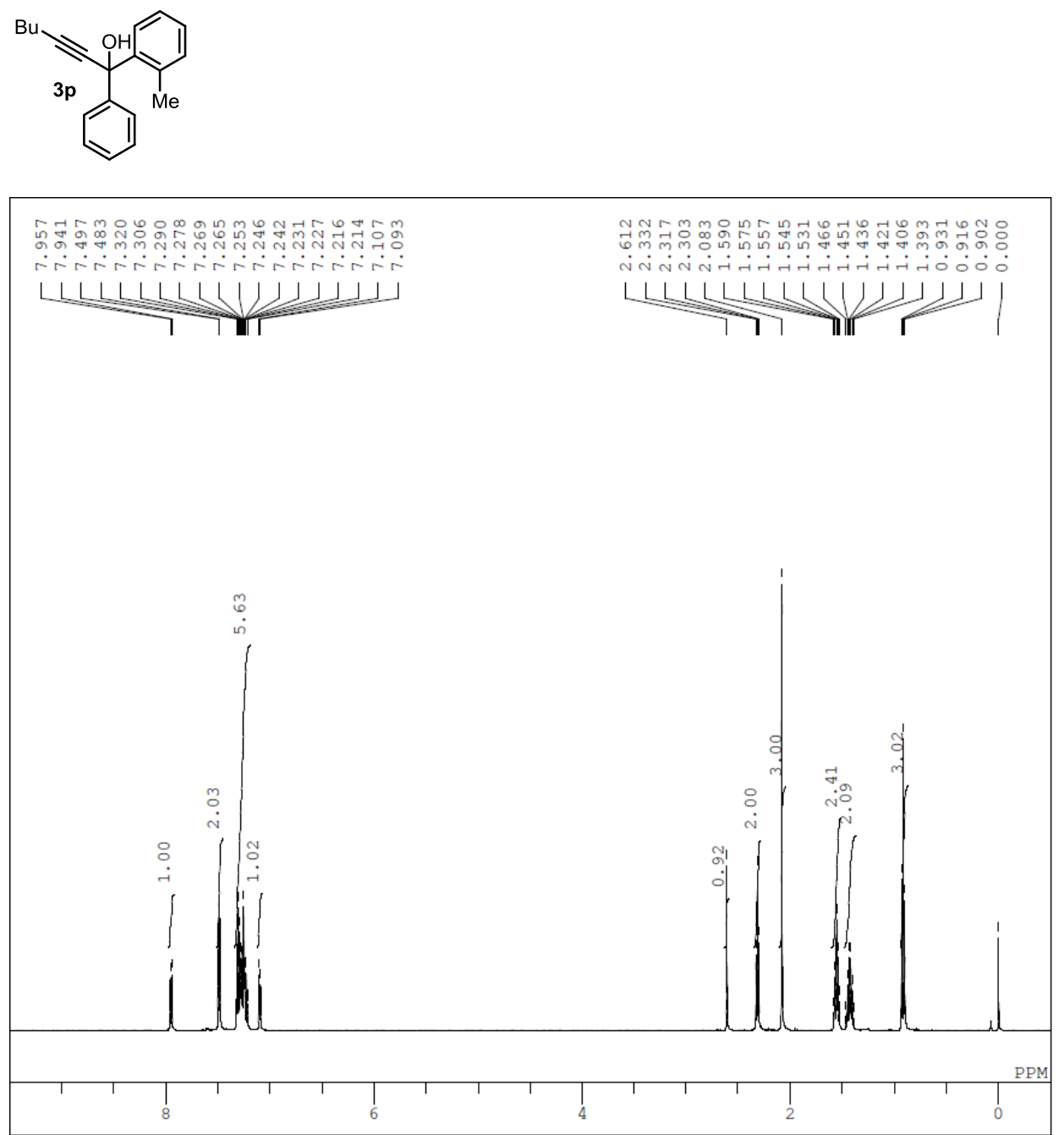

DFILE _Ym_06_111_2-1-1.als

COMNT single_pulse

DATIM 2015-02-07 15:55:18

OBNUC $1 \mathrm{H}$

EXMOD proton.jxp

OBFRQ $\quad 500.16 \mathrm{MHz}$

OBSET $2.41 \mathrm{KHz}$

OBFIN $\quad 6.01 \mathrm{~Hz}$

13107
FREQU $\quad 7507.51 \mathrm{~Hz}$

8
SCANS

ACQTM $\quad 1.7459 \mathrm{sec}$

PD $\quad 5.0000 \mathrm{sec}$

IRNUC $1 \mathrm{H}$

CTEMP $17.6 \mathrm{C}$

EXREF

$0.00 \mathrm{ppm}$

RGAIN $0.10 \mathrm{~Hz}$

DFILE -ym_06_111_2_13C-1-1.als

COMNT single pulse decoupled gated

DATIM 2015-02-07 16:05:51

DBNUC $13 \mathrm{C}$

EXMOD carbon.jxp

OBFRQ $\quad 125.77 \mathrm{MHz}$

OBSET $\quad 7.87 \mathrm{KHz}$

OBFIN $4.21 \mathrm{~Hz}$

OBFIN

POINT

FREQU

SCANS

$\mathrm{PD}$ $31446.54 \mathrm{~Hz}$

126

2.0000 sec

PW1 3.12 usec

IRNUC $1 \mathrm{H}$

SLVNT

BF
RGAIN

$17.9 \mathrm{C}$

CDCL 3

$77.00 \mathrm{ppm}$

$0.10 \mathrm{~Hz}$ 
TBSO_

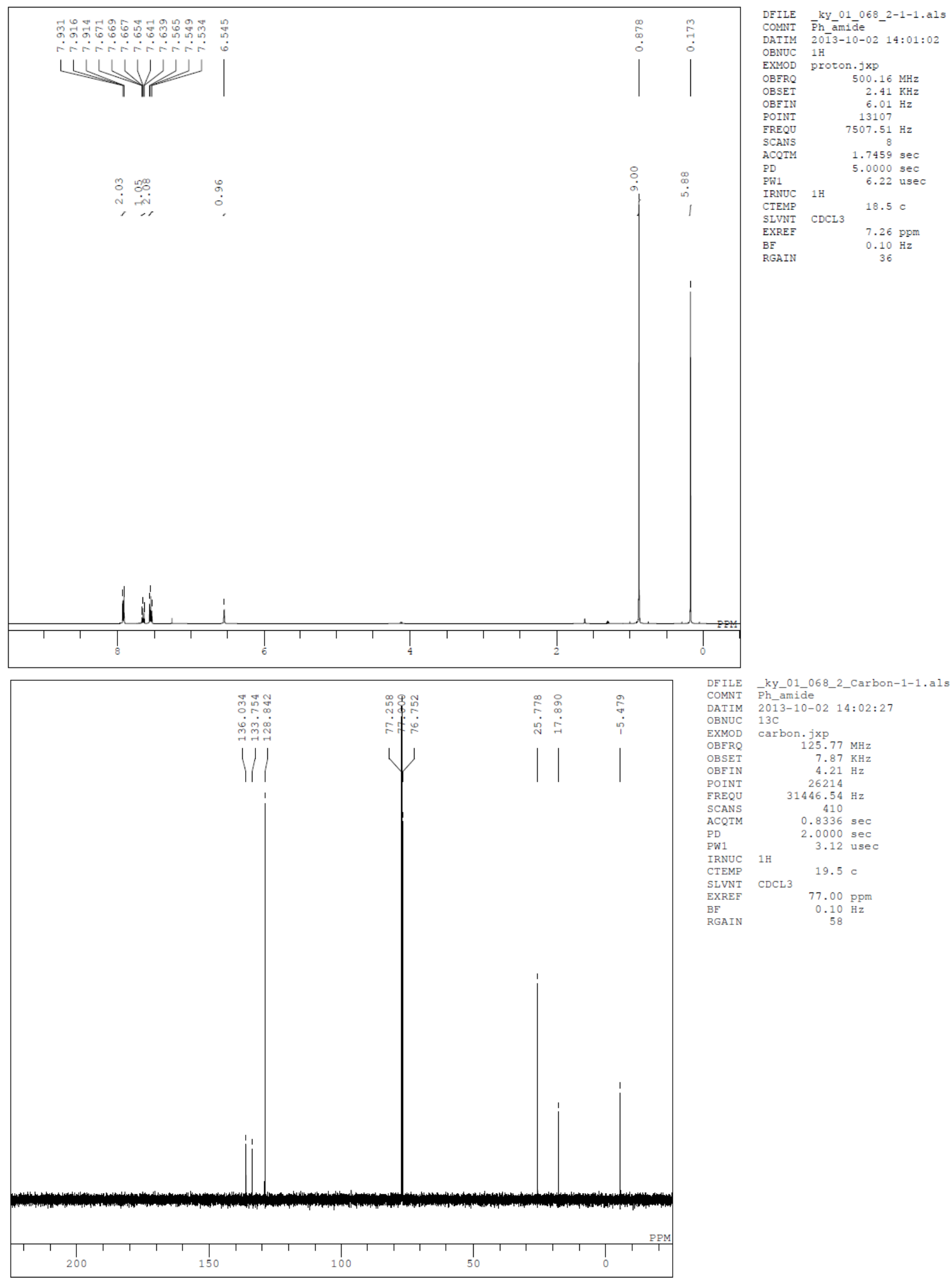


<smiles>CC(C)[R5]ONS(=O)(=O)c1ccc(Cl)cc1</smiles>

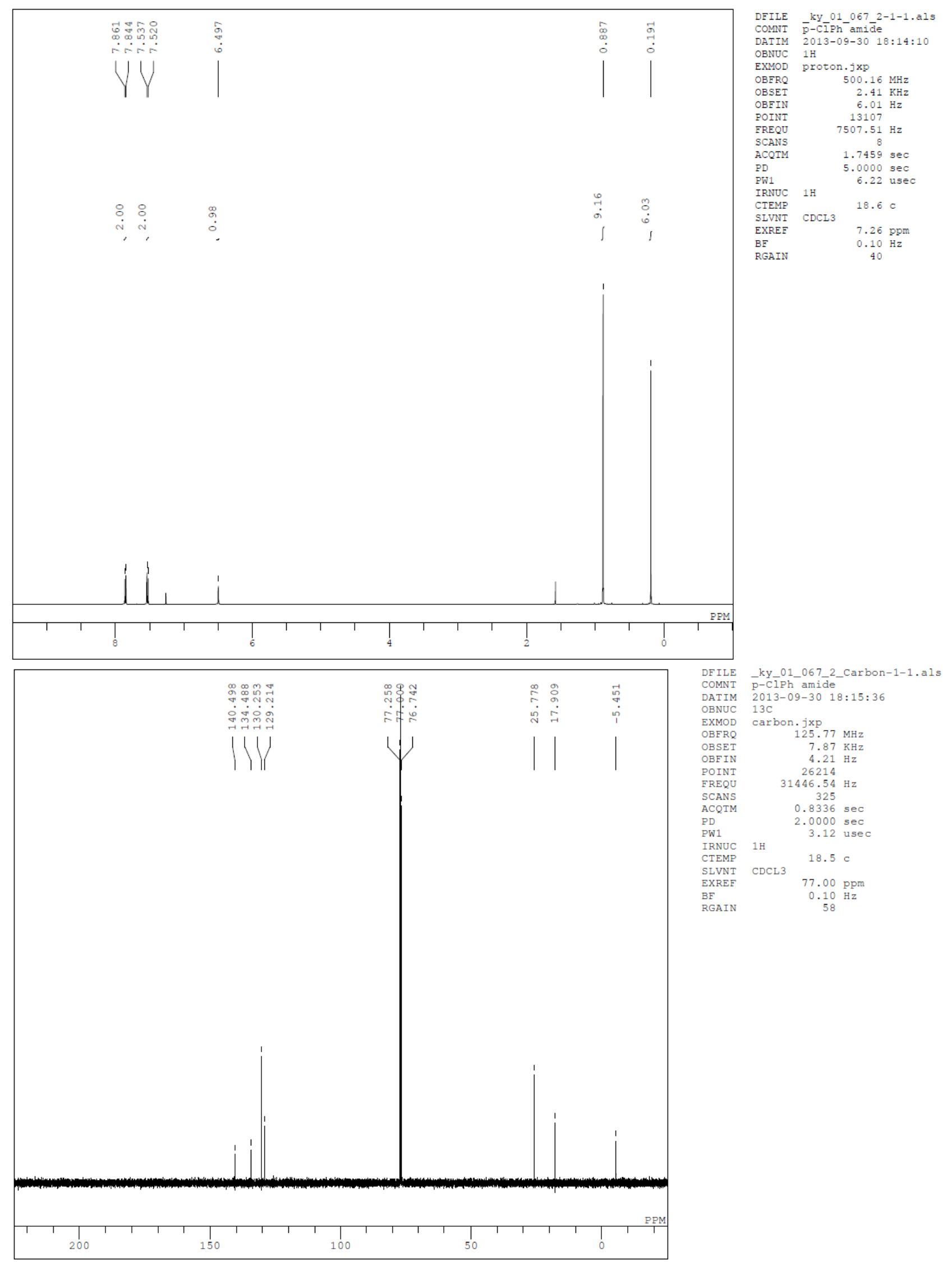


<smiles>CC(C)(C)ONS(=O)(=O)c1ccc(F)cc1</smiles>

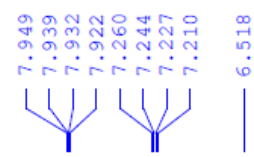

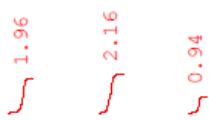
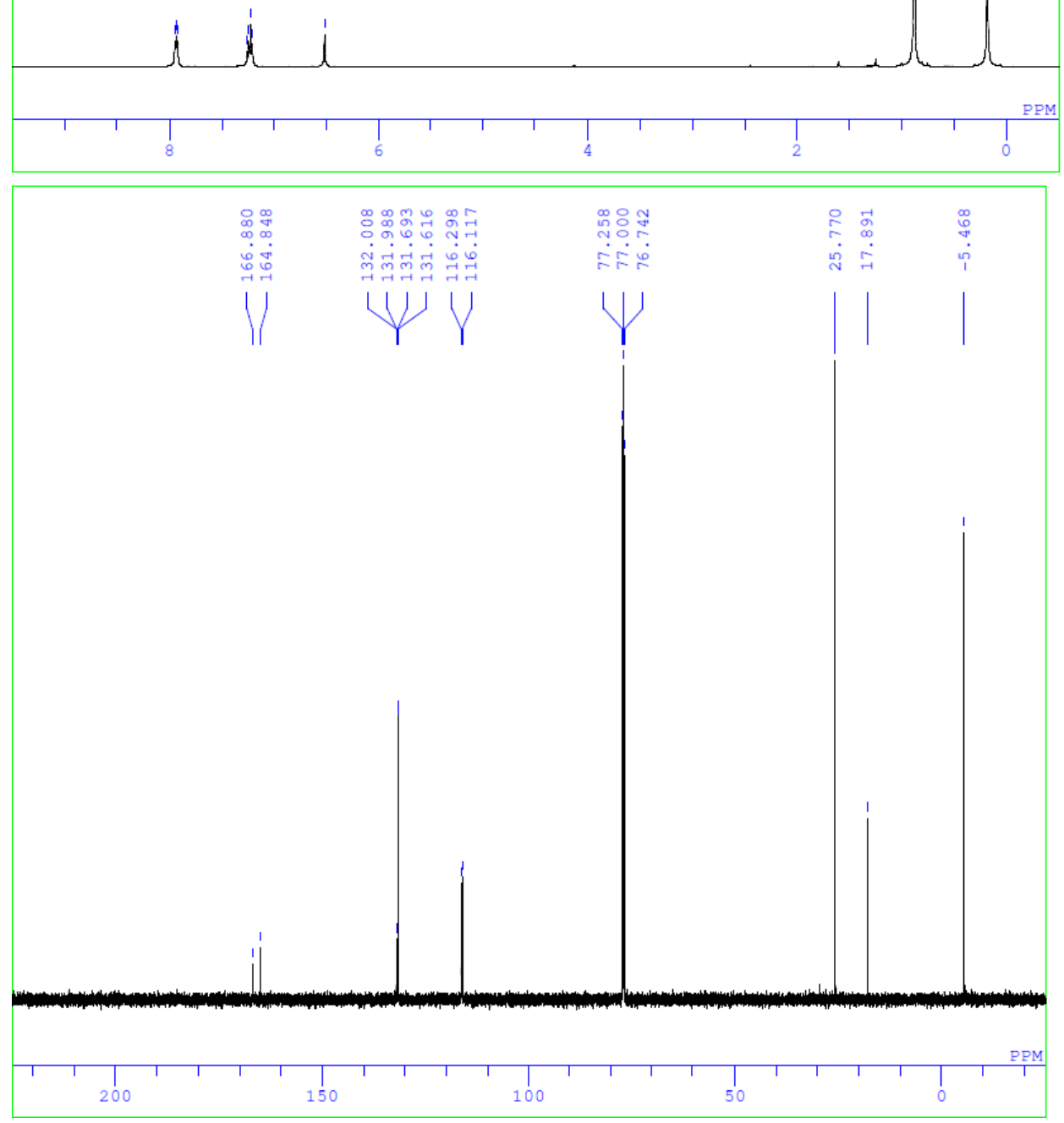

DFILE -ky_FPh_amide-1-1.jdf

DAMIM

DATIM 2015-01-29-12:34:15

OBNUC $1 \mathrm{H}$

$\begin{array}{lr}\text { EXMOD } & \text { proton. jxp } \\ \text { OBFRQ } & 500.16 \mathrm{MHz}\end{array}$

$\begin{array}{lr}\text { OBFRQ } & 500.16 \mathrm{MHZ} \\ \text { OBSET } & 2.41 \mathrm{KHz}\end{array}$

OBFIN $6.01 \mathrm{~Hz}$

POINT 16384

FREQU $9384.38 \mathrm{~Hz}$

SCANS 8

ACQTM $\quad 1.7459 \mathrm{sec}$

PD $\quad 5.0000 \mathrm{sec}$

PW1 6.22 usec

$\begin{array}{lll}\text { IRNUC } & \text { H H } & \\ \text { CTEMP } & & 16.5 \mathrm{C}\end{array}$

SLVNT CDCL3

EXREF $7.26 \mathrm{ppm}$

$\begin{array}{ll}\text { BF } & 0.10 \mathrm{~Hz}\end{array}$

DFILE_ky_FPh_amide_carbon-1-1.jdf

COMNT ky_FPh amide

DATIM 2015-01-29 12:36:08

OBNUC $13 \mathrm{C}$

EXMOD carbon. jXP

OBFRQ $\quad 125.77 \mathrm{MHz}$

OBSET $\quad 7.87 \mathrm{KHz}$

32767

FREQU $\quad 39308.18 \mathrm{~Hz}$

SCANS 600

ACQTM $\quad 0.8336 \mathrm{sec}$

PD $2.0000 \mathrm{sec}$

PW1 3.12 usec

IRNUC $1 \mathrm{H}$

SLVNT CDCL3

EXREF $\quad 77.00 \mathrm{ppm}$

BF $\quad 0.10 \mathrm{~Hz}$ 
<smiles>CC(C)CONS(=O)(=O)c1ccc(F)cc1</smiles>

4d ${ }_{19} \mathrm{~F}$ NMR (hexafluorobenzene as standard: $\delta-162.0 \mathrm{ppm}$ )

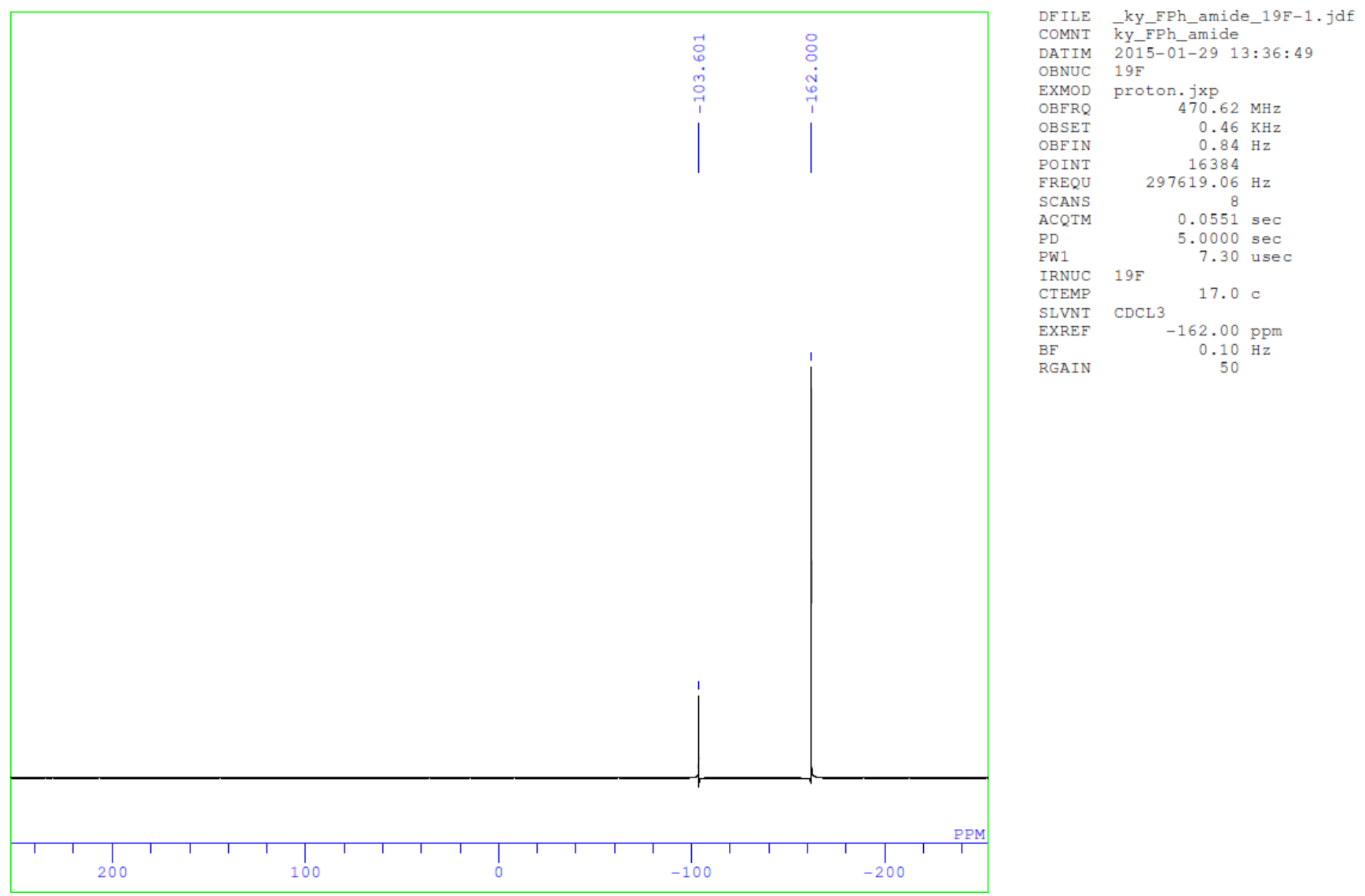


<smiles>COc1ccc(S(=O)(=O)NOC(C)(C)C)cc1</smiles>
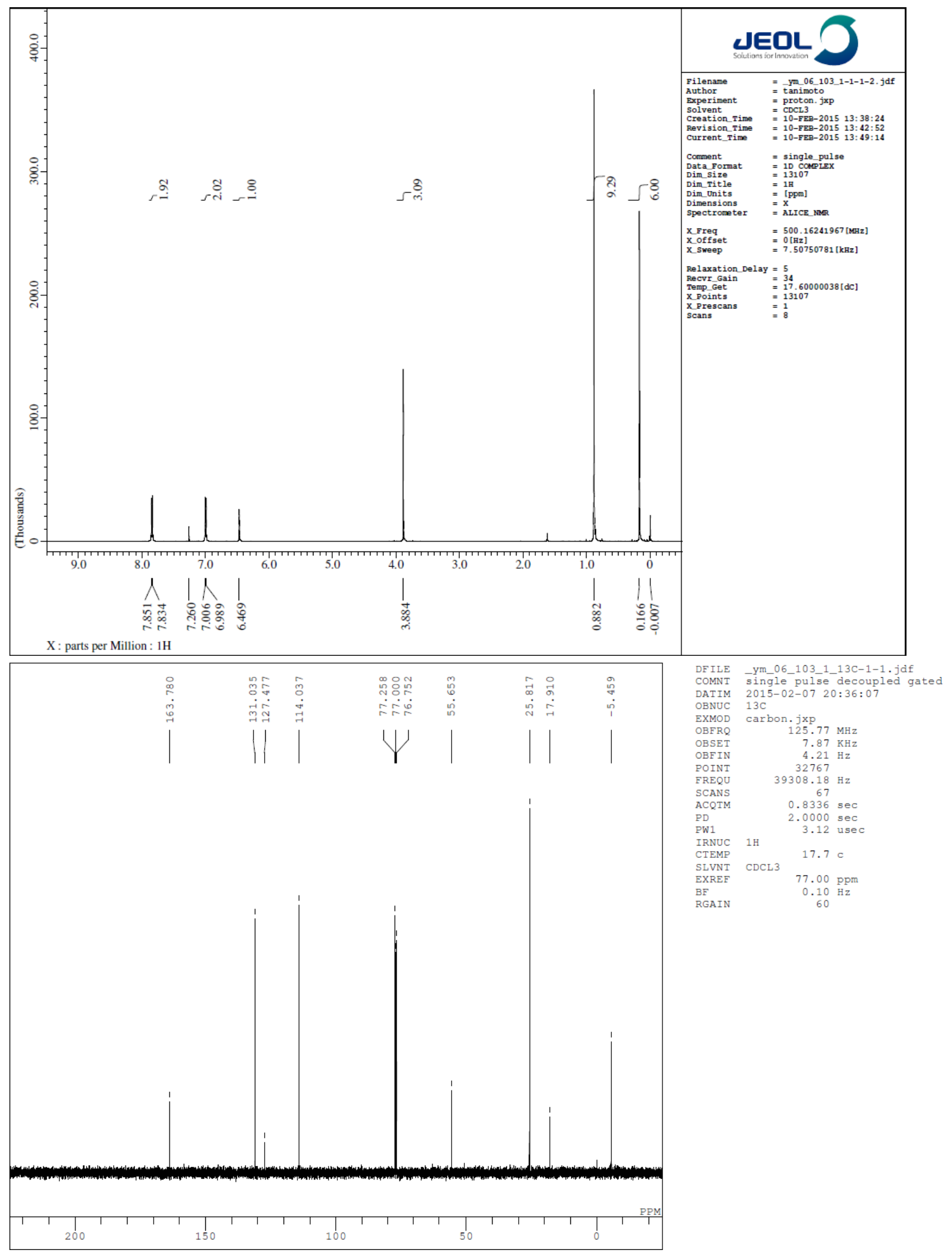


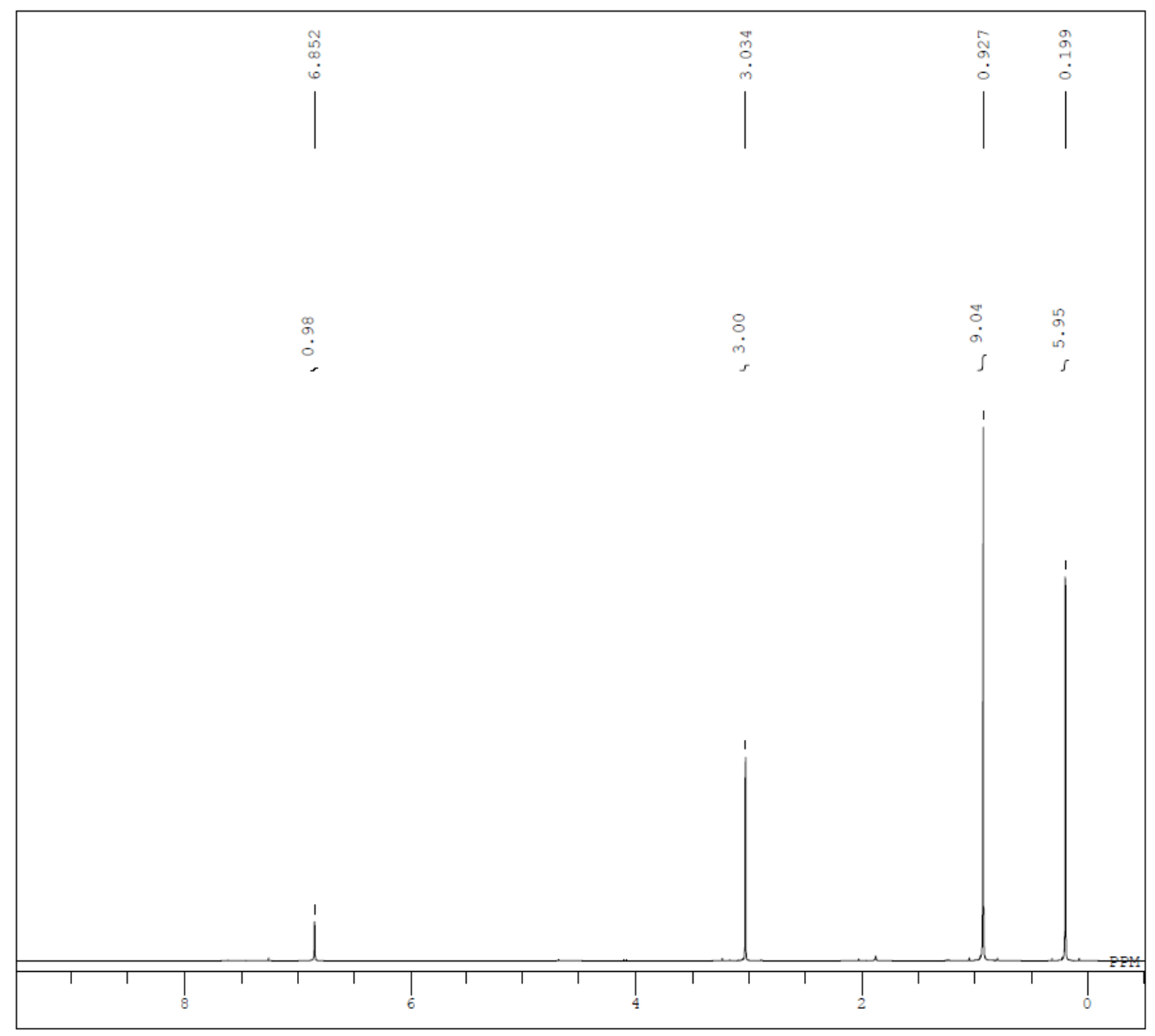

DFILE Ky_01_066_5-1-1.als

COMNT Ms_allenamide

DATIM 2013-10-16 10:29:5

EBNOC 1

EXMOD proton.jxp

OBFRQ $\quad 500.16 \mathrm{MHz}$

OBSET $2.41 \mathrm{KHz}$

OBEIN $6.01 \mathrm{~Hz}$

$\begin{array}{lr}\text { POINI } & 13107 \\ \text { FREQU } & 7507.51 \mathrm{~Hz}\end{array}$

$7507.51 \mathrm{~Hz}$
SCANS

ACQTM $1.7459 \mathrm{sec}$

PD $\quad 5.0000 \mathrm{sec}$

PW1 $\quad 6.22$ use

IRNUC
CTEMP

SLVNT CDCL3 3 .

EXREF $\quad 7.26 \mathrm{ppm}$

$\begin{array}{lc}\text { BF } & 0.10 \mathrm{~Hz} \\ \text { RGAIN } & 24\end{array}$

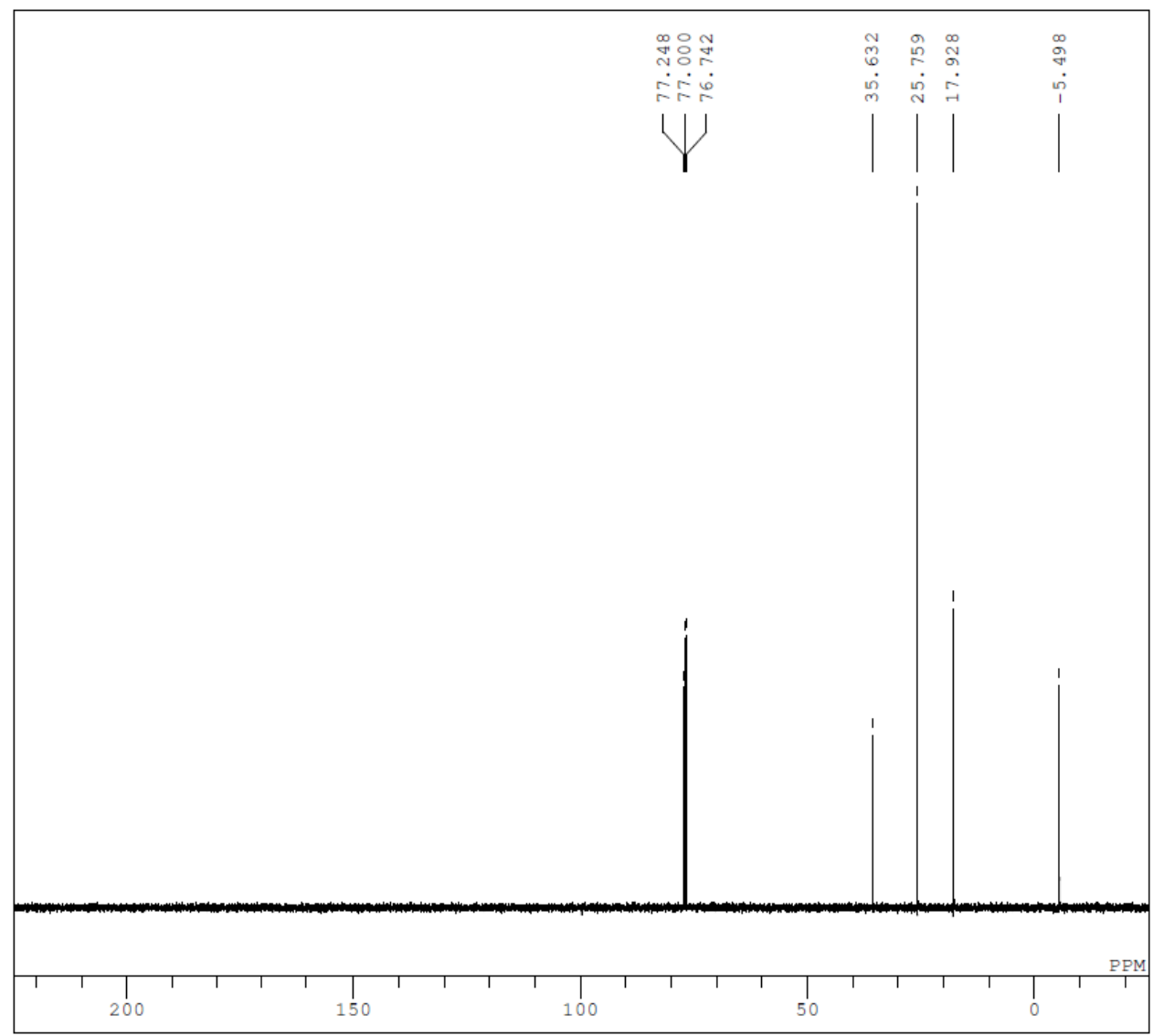

DFILE _ky_01_066_5_Carbon-1-1

COMNT Ms allenamide

DATIM 2013-10-16 10:32:33

BNUC $13 \mathrm{C}$

EXMOD carbon. jXP

OBSET $7.87 \mathrm{KHz}$

OBFIN

POINT 26214

FREQU $\quad 31446.54 \mathrm{~Hz}$

CATM

1446.54

$0.8336 \mathrm{sec}$

$\begin{array}{lr}\text { PD } & 2.0000 \mathrm{sec} \\ \text { PW1 } & 3.12 \text { usec }\end{array}$

IRNUC $1 \mathrm{H}$

CTEMP

SLVNT CDCL 3

EXREF

$\mathrm{BF}$

$17.7 \mathrm{c}$

CDCL3

$77.00 \mathrm{ppm}$

RGAIN

$0.10 \mathrm{~Hz}$ 
<smiles>Cc1cc(C)c(S(=O)(=O)NOC(C)(C)C)c(C)c1</smiles>
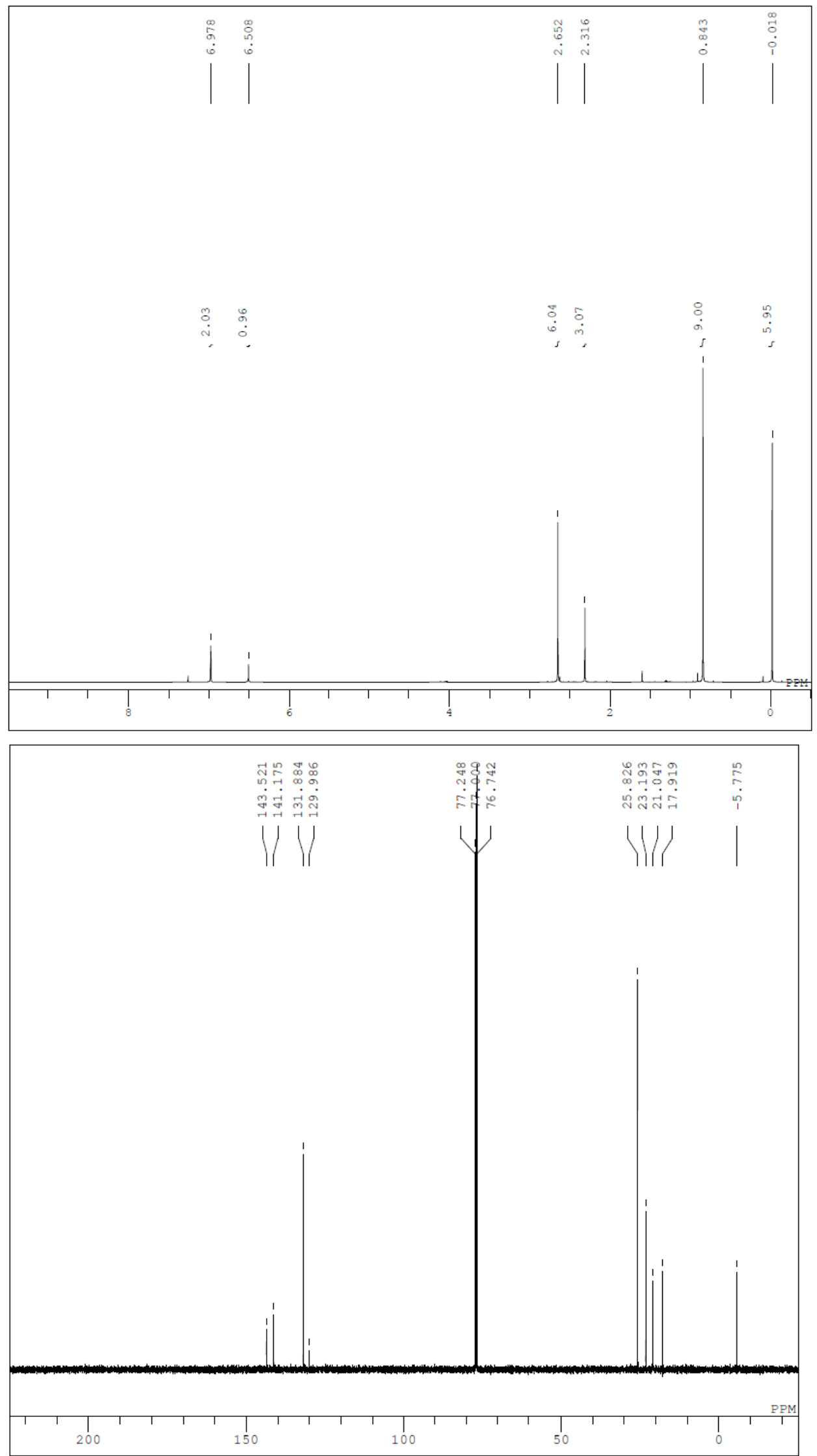
<smiles>CCCCC(C)ON([TeH])C(=C=C(c1ccccc1)c1ccccc1)c1ccccc1</smiles>
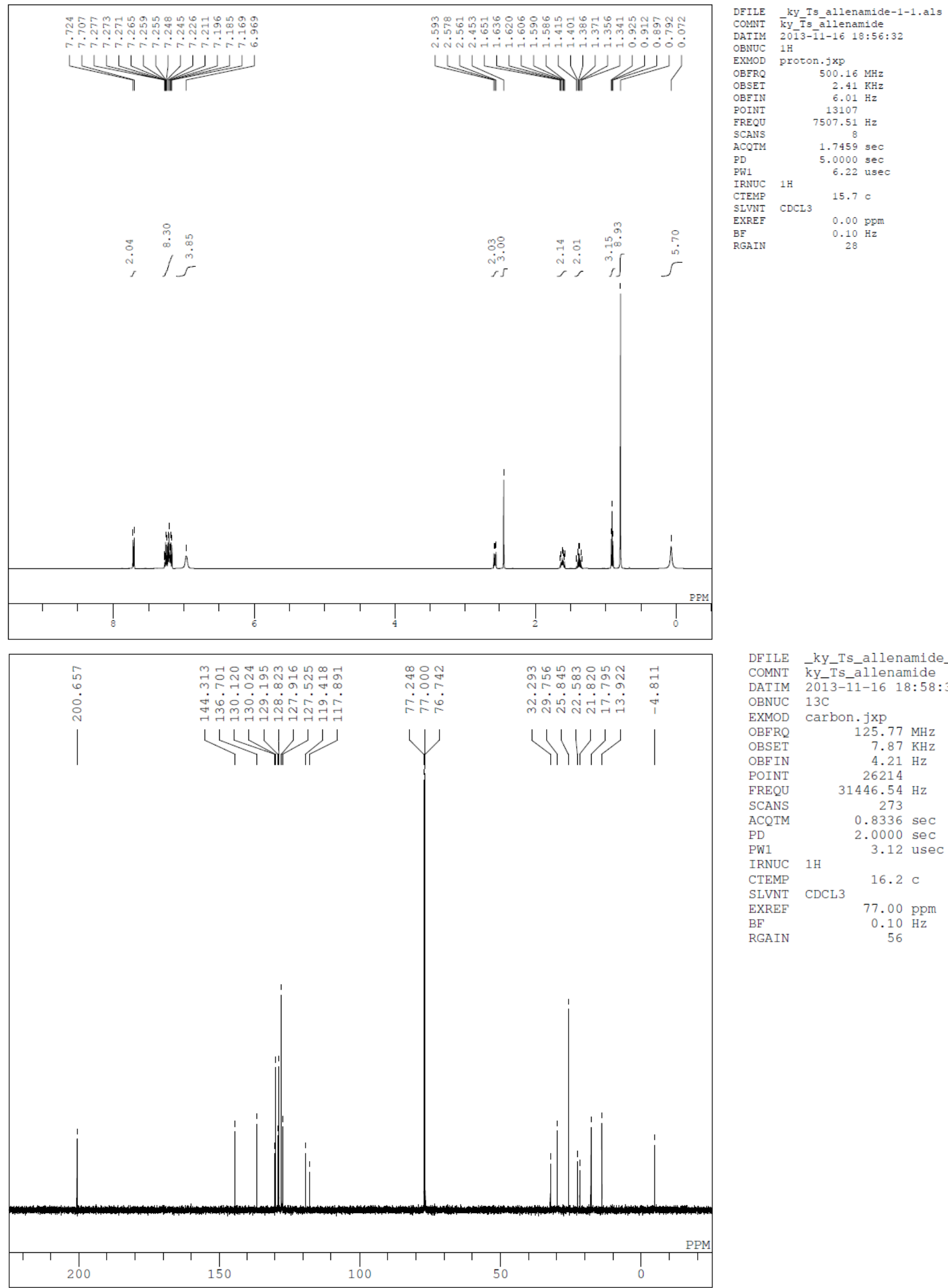

DFILE

COMNT ky Ts allenamide

DATTM 2013-11-16 18:58:34

OBNUC 13C

EXMOD carbon.jX

OBFRQ $\quad 125.77 \mathrm{MHz}$

$\begin{array}{lr}\text { OBSET } & 7.87 \mathrm{KHz}\end{array}$

OBFIN

POINT

REQU

SCANS

$\mathrm{ACQTM}$

$\mathrm{PD}$ $4.21 \mathrm{~Hz}$ 26214

$31446.54 \mathrm{~Hz}$ 273

$0.8336 \mathrm{sec}$

$2.0000 \mathrm{sec}$

PW1 3.12 usec

IRNUC $1 \mathrm{H}$

CTEMP

SLVNT CDCL3

EXREF

BE

$16.2 \mathrm{C}$

RGAIN

$77.00 \mathrm{ppm}$

$0.10 \mathrm{~Hz}$

56 

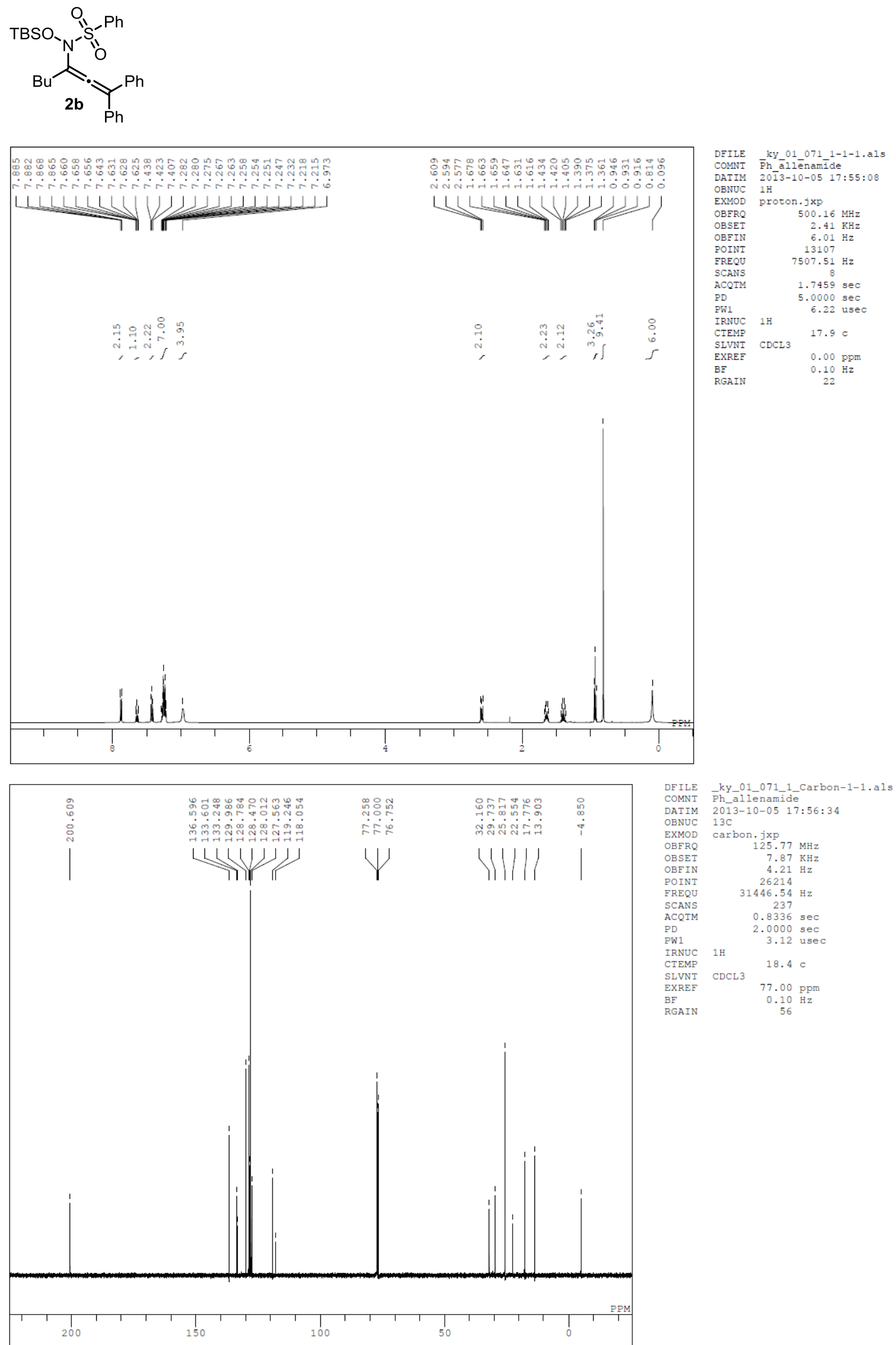
<smiles>CC(C)[C@H](Br)ON(C(Br)=CC(=O)c1ccccc1)S(=O)(=O)c1ccc(Cl)cc1</smiles>

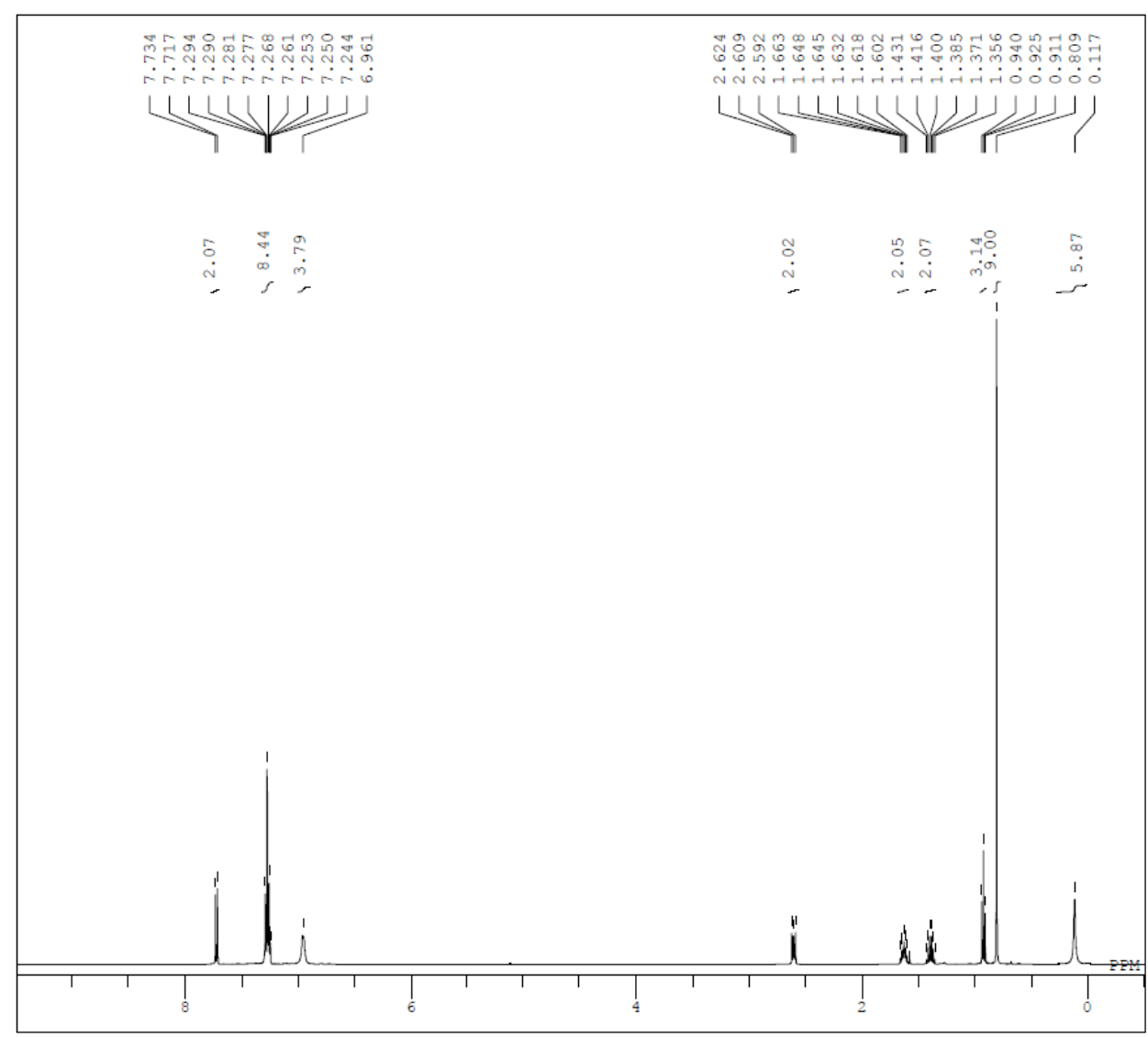

DFILE ky 01 070 s1-1-1.al

CoMi p-Cirh Allenamide.

DATM 2013-10-02 14:34:59

OBNUC 1

$\begin{array}{lc}\text { EXMOD } & \text { proton.jxp } \\ \text { OBFRQ } & 500.16 \mathrm{MHz}\end{array}$

OBSET $\quad 2.41 \mathrm{KHz}$

$\begin{array}{lr}\text { OBEIN } & 6.01 \mathrm{~Hz} \\ \text { POINI } & 13107\end{array}$

FREQU $\quad 7507.51 \mathrm{~Hz}$

$\begin{array}{lr}\text { SCANS } & 8 \\ \text { ACQTM } & 1.7459 \mathrm{sec}\end{array}$

5.0000 sec

PD1 $\quad 5.0000 \mathrm{sec}$

IRNUC $1 \mathrm{H}$

SIEMNT CDCL 3

EXRE

RGAIN $\quad 0.10 \mathrm{~Hz}$

30

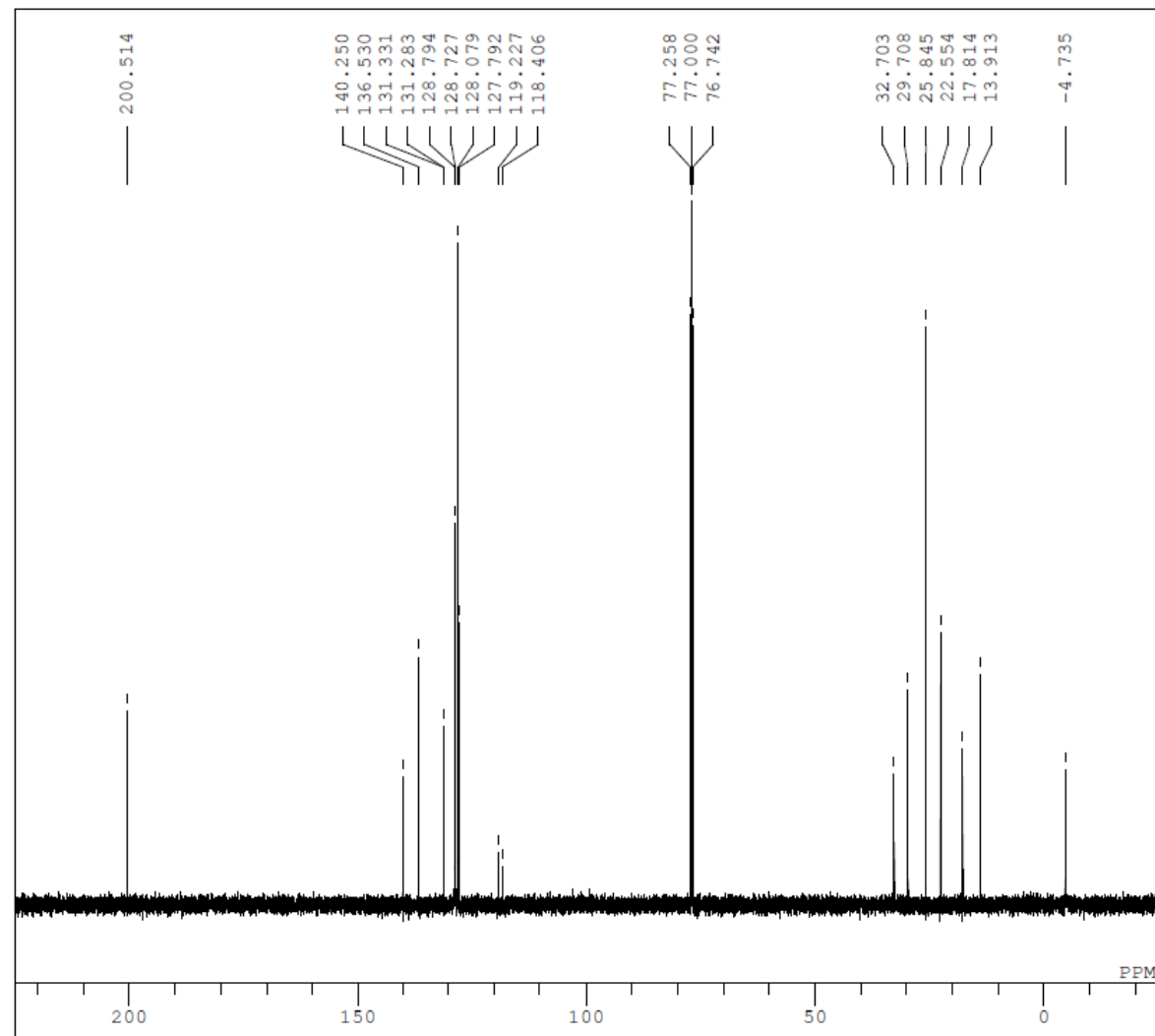

DFILE ky_01_070 s1 Carbon-1-1.a1s

COMNT p-Clph Allenamide

DATIM 2013-10-02 14:36:25

OBNUC $13 \mathrm{C}$

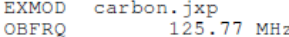

OBFRQ $\quad 125.77 \mathrm{MHz}$

$\begin{array}{ll}\text { OBSET } & 7.87 \mathrm{KHz} \\ \text { OBETN } & 4.21 \mathrm{~Hz}\end{array}$

OBFIN $\quad 4.21 \mathrm{~Hz}$

$\begin{array}{lr}\text { POINT } & 26214 \mathrm{~Hz} \\ \text { FREQU } & 31446.54 \mathrm{~Hz}\end{array}$

$\begin{array}{lr}\text { FREQU } & 31446.54 \mathrm{~Hz} \\ \text { SCANS } & 299\end{array}$

ACANS $\quad 0.8336 \mathrm{sec}$

PD $2.0000 \mathrm{sec}$

PW1 3.12 use

$\begin{array}{lll}\text { IRNUC } & 1 \mathrm{H} & 19.6 \text { 。 } \\ \text { CTEMP } & & \end{array}$

SLVNT CDCL 3

EXREF $\quad 77.00 \mathrm{ppm}$

BF $0.10 \mathrm{~Hz}$ . 
<smiles>CC(C)(C)CN(C(Br)=CC(=O)c1ccccc1)S(=O)(=O)c1ccc(F)cc1</smiles>
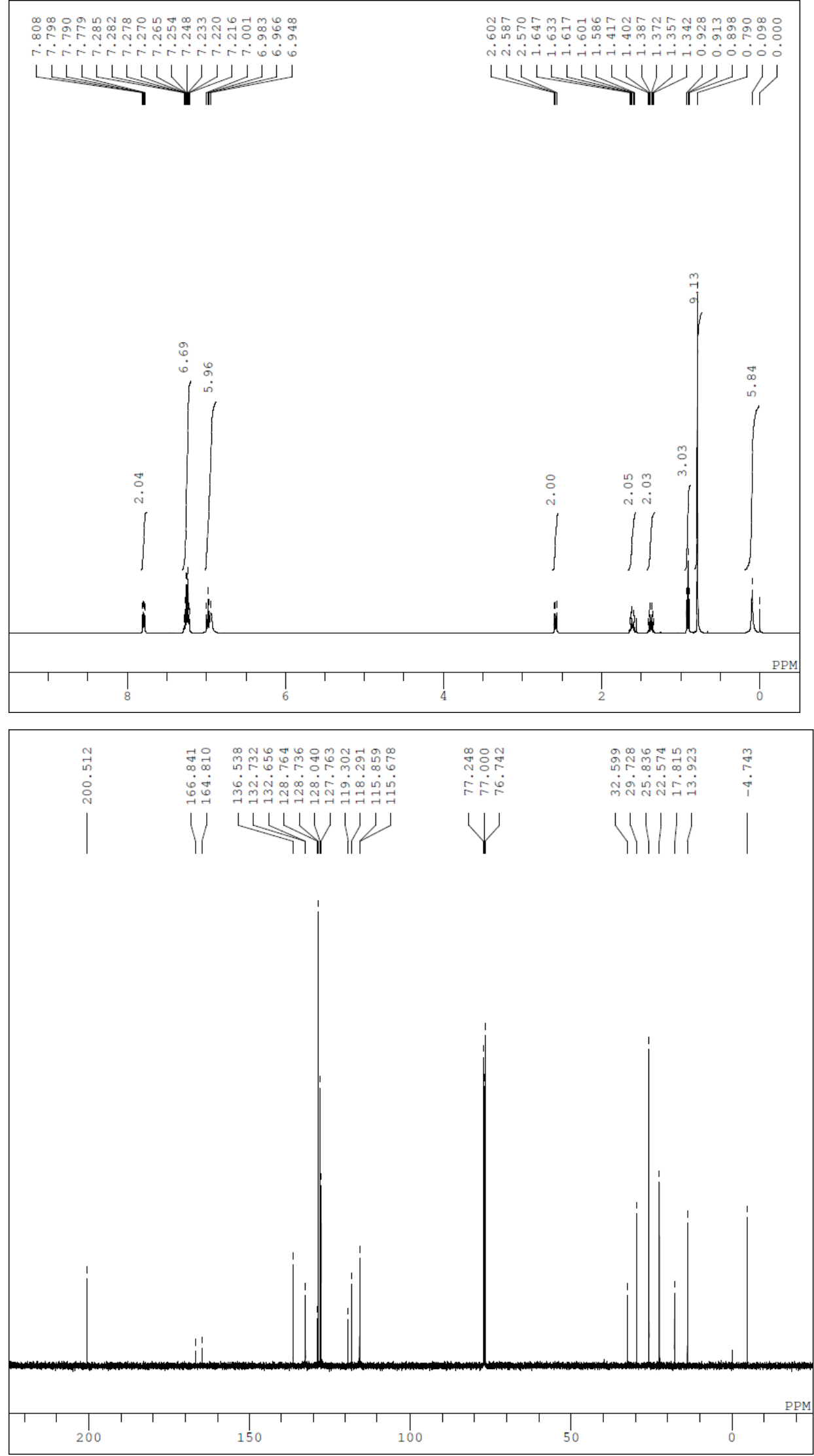

DFILE _ym_06_018_3-1-1.jdf

(1)

DATIM 2015-02-14 14:23:48

XMOD proton.jxp

OBFRQ $\quad 500.16 \mathrm{MHz}$

OBSET $2.41 \mathrm{KHZ}$

OBFIN $\quad 6.01 \mathrm{~Hz}$

FREQU $\quad 9384.38 \mathrm{~Hz}$

SCANS

SCANS

PD

8
1.7459
$5 e c$

5.0000 sec

$5.0000 \mathrm{sec}$

IRNUC $1 \mathrm{H}$

IRNUC $1 \mathrm{H}$

SLVNT CDCL 3

EXREF

EXR
BF

BFAIN

$0.00 \mathrm{ppm}$

$0.10 \mathrm{~Hz}$

DFILE -ym_06_018_3_13C-1-1.jdf

COMNT single pulse decoupled gated

DATIM 2015-02-14 14:25:12

OBNUC 13C

EXMOD carbon jxp

OBFRQ $\quad 125.77 \mathrm{MHz}$

125.77
OBSET $\quad 7.87 \mathrm{KHz}$

OBFIN $4.21 \mathrm{~Hz}$

POINT 32767

FREQU $\quad 39308.18 \mathrm{~Hz}$

SCANS 370

ACOTM

$0.8336 \mathrm{sec}$

PD $2.0000 \mathrm{sec}$

IRNUC $1 \mathrm{H}$

$\begin{array}{lll}\text { IRNUC } & \mathrm{H} & \mathrm{H} \\ \text { CTEMP } & & \end{array}$

SLVNT CDCL 3

EXREF $77.00 \mathrm{pmm}$

$\begin{array}{lr}\text { BF } & 77.00 \mathrm{ppm} \\ & 0.10 \mathrm{~Hz}\end{array}$

$.10 \mathrm{H}$ 
<smiles>CC(C)(C)ON(C(Br)=CC(=O)c1ccccc1)S(=O)(=O)c1ccc(F)cc1</smiles>

${ }^{19} \mathrm{~F}$ NMR (hexafluorobenzene as standard: $\delta-162.0 \mathrm{ppm}$ )

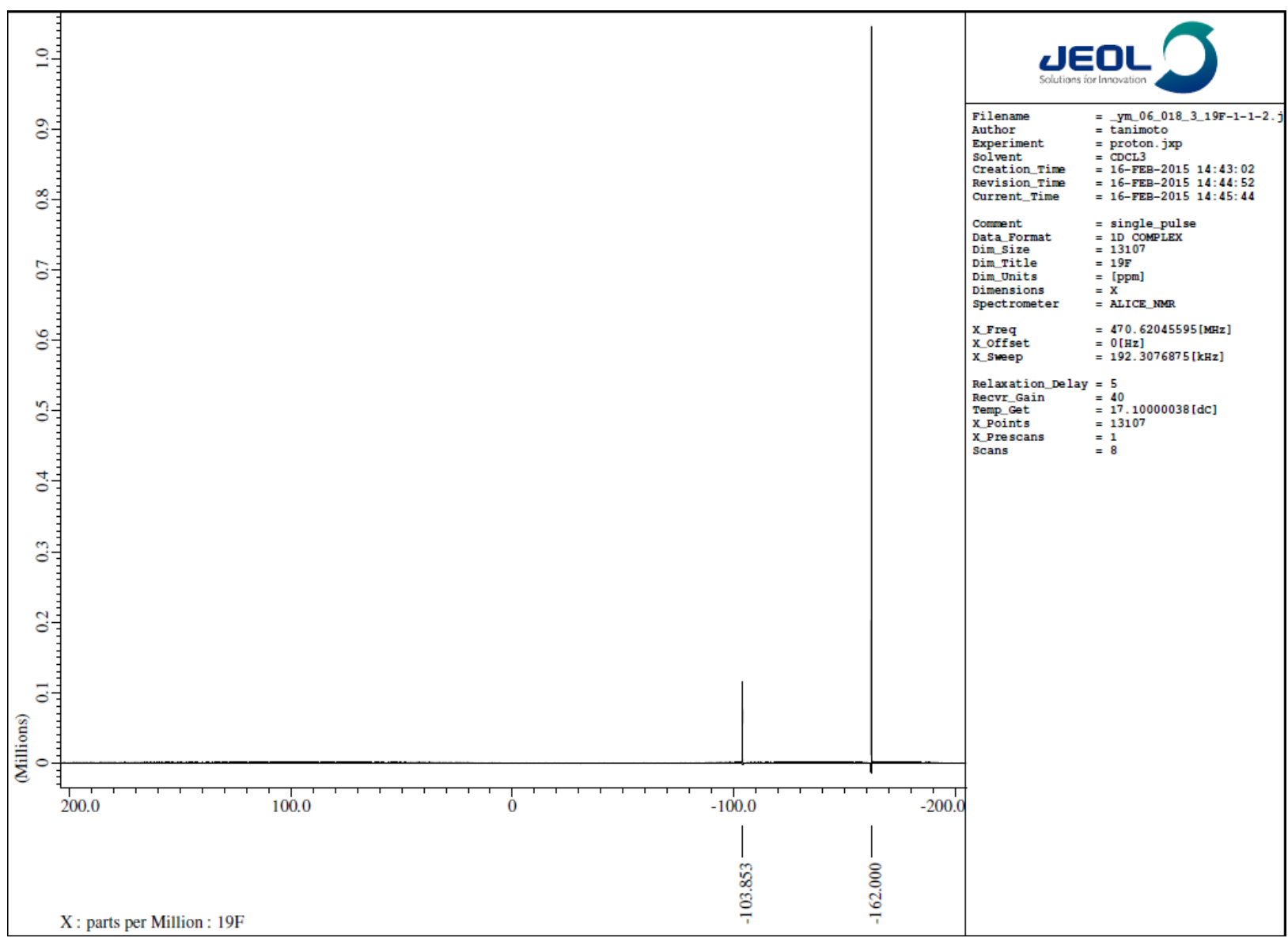




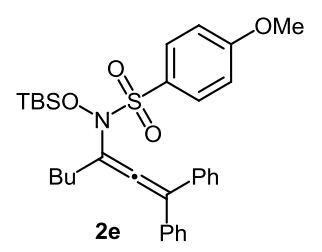

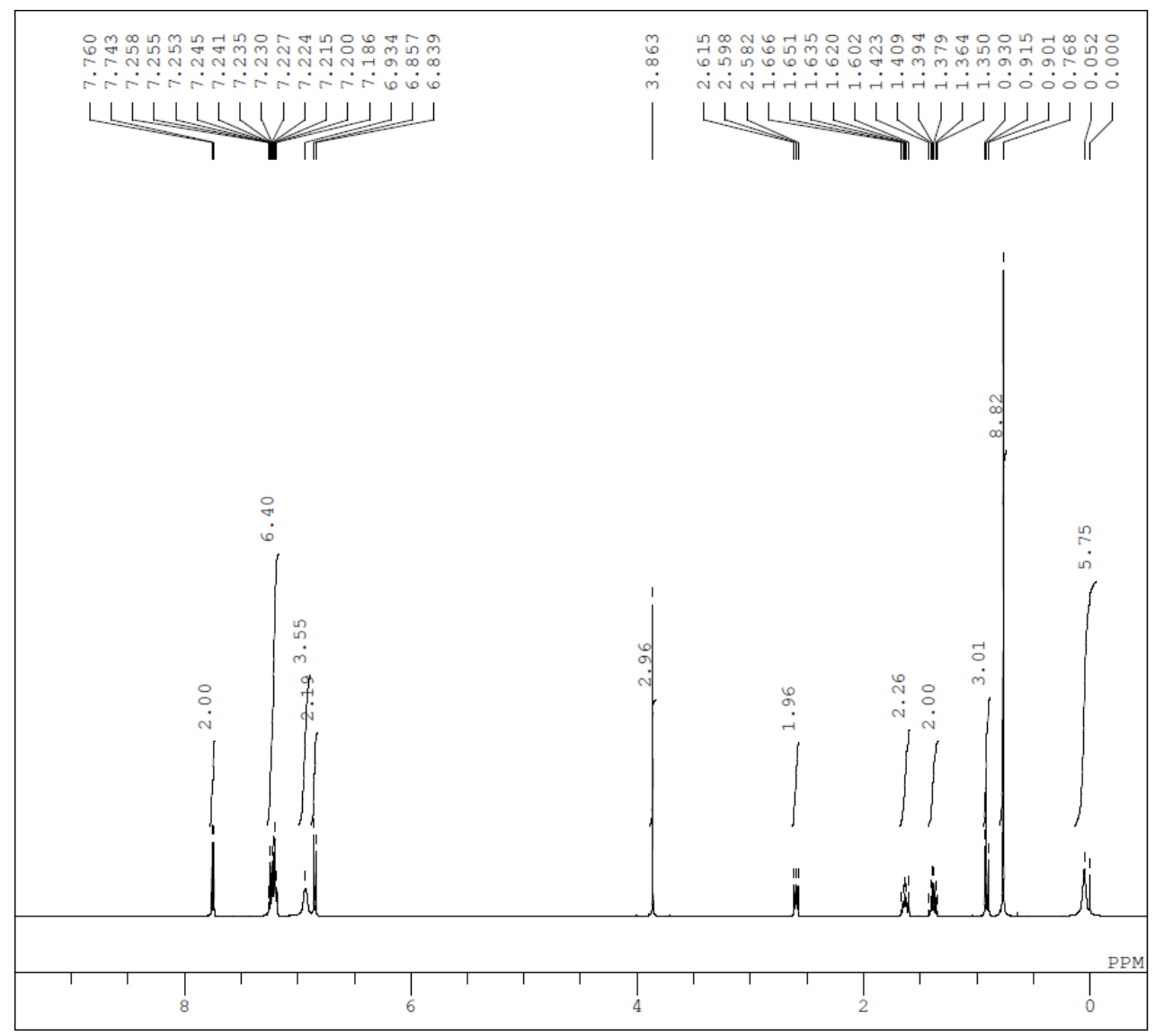

DFILE -ym_06_011_3-1-1.als

COMNT single_pulse

DATIM 2014-09-01 21:13:20

OBNUC $1 \mathrm{H}$

$\begin{array}{lc}\text { EXMOD } & \text { proton.jxp } \\ \text { OBFRQ } & 500.16 \mathrm{MHz}\end{array}$

OBSET $2.41 \mathrm{KHz}$

OBFIN $\quad 6.01 \mathrm{~Hz}$

$\begin{array}{lr}\text { POINT } & 13107\end{array}$

FREQU $\quad 7507.51 \mathrm{~Hz}$

$\begin{array}{lr}\text { SCANS } & 8 \\ \text { ACOTM } & 1.7459 \mathrm{sec}\end{array}$

$5.0000 \mathrm{sec}$

PW1 1 6.02

IRNUC $1 \mathrm{H}$

CTEMP 1 H 18.6 C

SLVNT CDCL3

SLVNT CDCL3 0.00 ppm

$\begin{array}{ll}\text { EXREF } & 0.00 \mathrm{ppm} \\ \text { BF } & 0.10 \mathrm{~Hz}\end{array}$

RGAIN 24

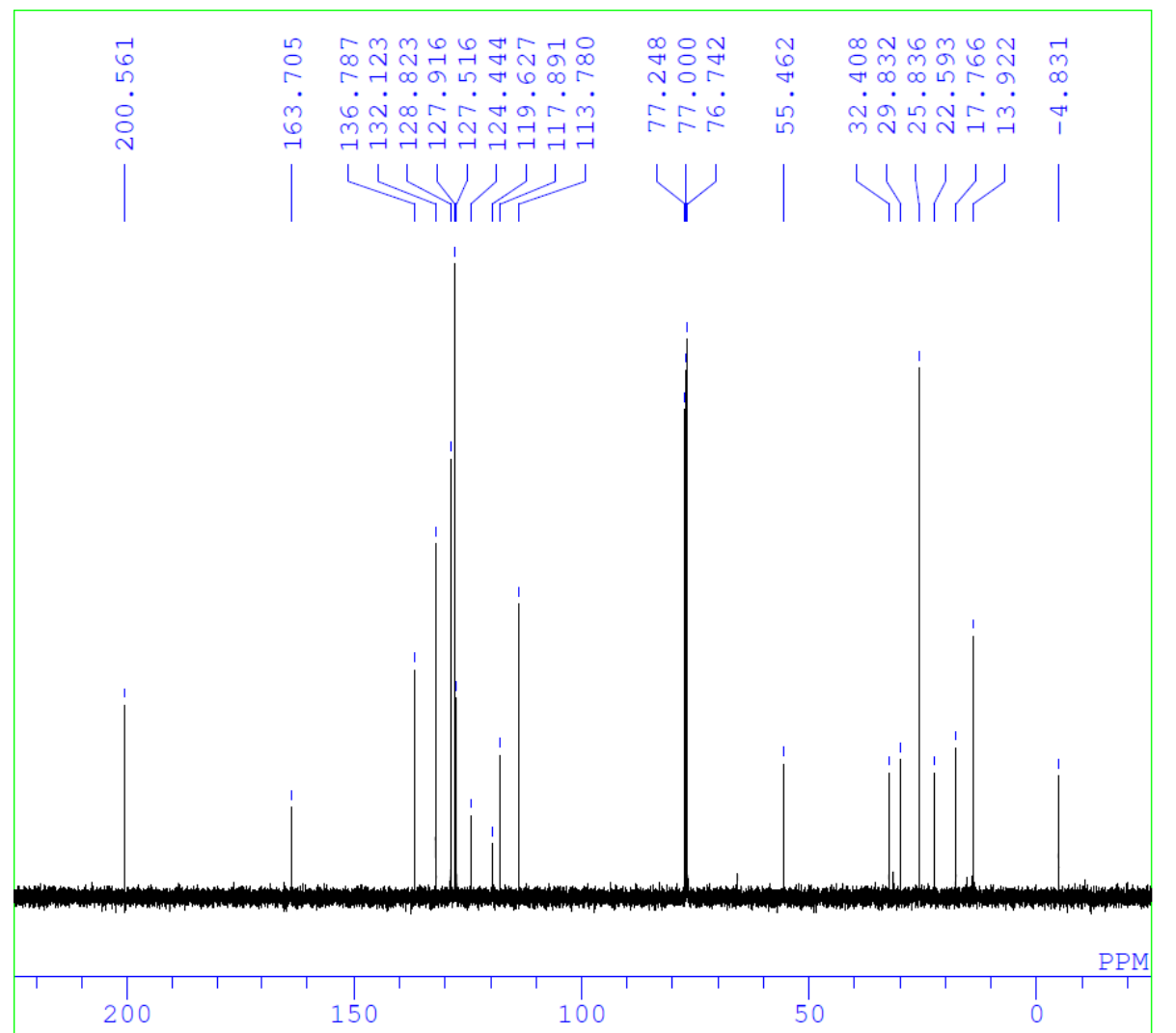

DFILE _ym_06_011_2_13C-1-

COMNT single pulse decoup

DATIM 2014-08-29 20:03:20

OBNUC $13 \mathrm{C}$

EXMOD carbon.jxp

OBFRQ $\quad 125.77 \mathrm{MHz}$

$\begin{array}{lr}\text { OBSET } & 7.87 \mathrm{KHz}\end{array}$

OBFIN $4.21 \mathrm{~Hz}$

POINT

FREQU

SCANS

ACQTM

$\mathrm{PD}$

PW1

IRNUC

CTEMP

SLVNT

EXREF

$\mathrm{BF}$

26214

$31446.54 \mathrm{~Hz}$ 82

$0.8336 \mathrm{sec}$

2.0000 sec

$1 \mathrm{H}$

3.12 usec

CDCL3

$19.8 \mathrm{C}$

RGAIN

$77.00 \mathrm{ppm}$

$0.10 \mathrm{~Hz}$

58 
<smiles>CC(C)ON(O[As])C(=CC(=[Te])c1ccccc1)c1ccccc1</smiles>

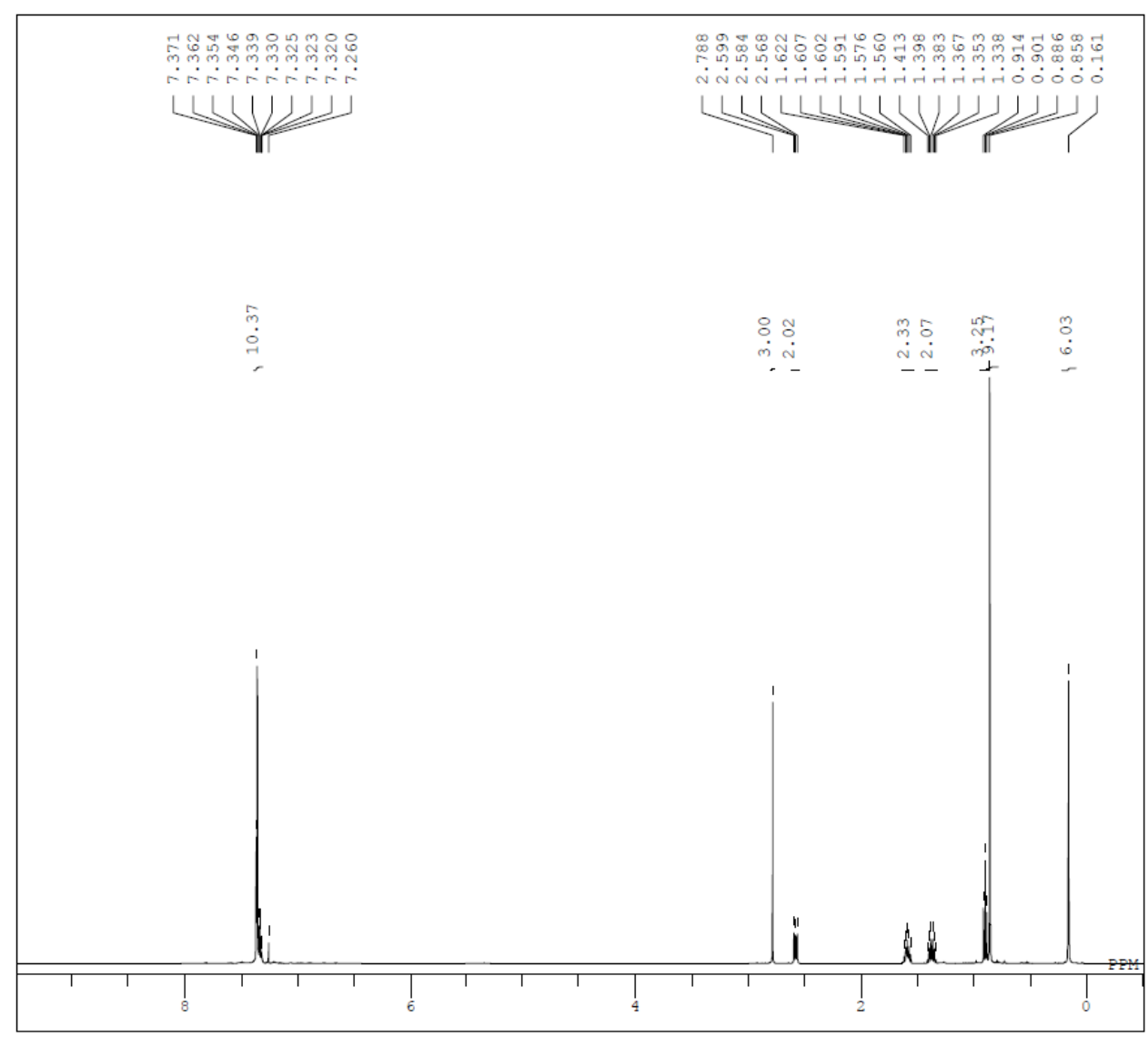

DFILE kY $01-^{066}-^{2-1-1 . a 1 s}$

COMNT Ms allenamide

DATIM 2013-10-16 01:51:57

$\begin{array}{rr}\text { OBFRQ } & 500.16 \mathrm{MHz} \\ \text { OBSET } & 2.41 \mathrm{KHz}\end{array}$

$\begin{array}{ll}\text { OBSET } & 2.41 \mathrm{KHz} \\ \text { OBFIN } & 6.01 \mathrm{~Hz}\end{array}$

POINT 13107

FREQU $\quad 7507.51 \mathrm{~Hz}$

SCANS $\quad 8$

$5.0000 \mathrm{sec}$

$\begin{array}{lr}\text { PD } & 5.0000 \mathrm{sec} \\ \text { PW1 } & 6.22 \text { usec }\end{array}$

$\begin{array}{ll}\text { IRNUC } & \text { H } \\ \text { CTEMP } & 18.2 \text { 。 }\end{array}$

SLVNT CDCL3

EXREF $\quad 7.26 \mathrm{ppm}$

$\begin{array}{lc}\text { BF } & 0.10 \mathrm{~Hz} \\ \text { RGAIN } & 26\end{array}$

26

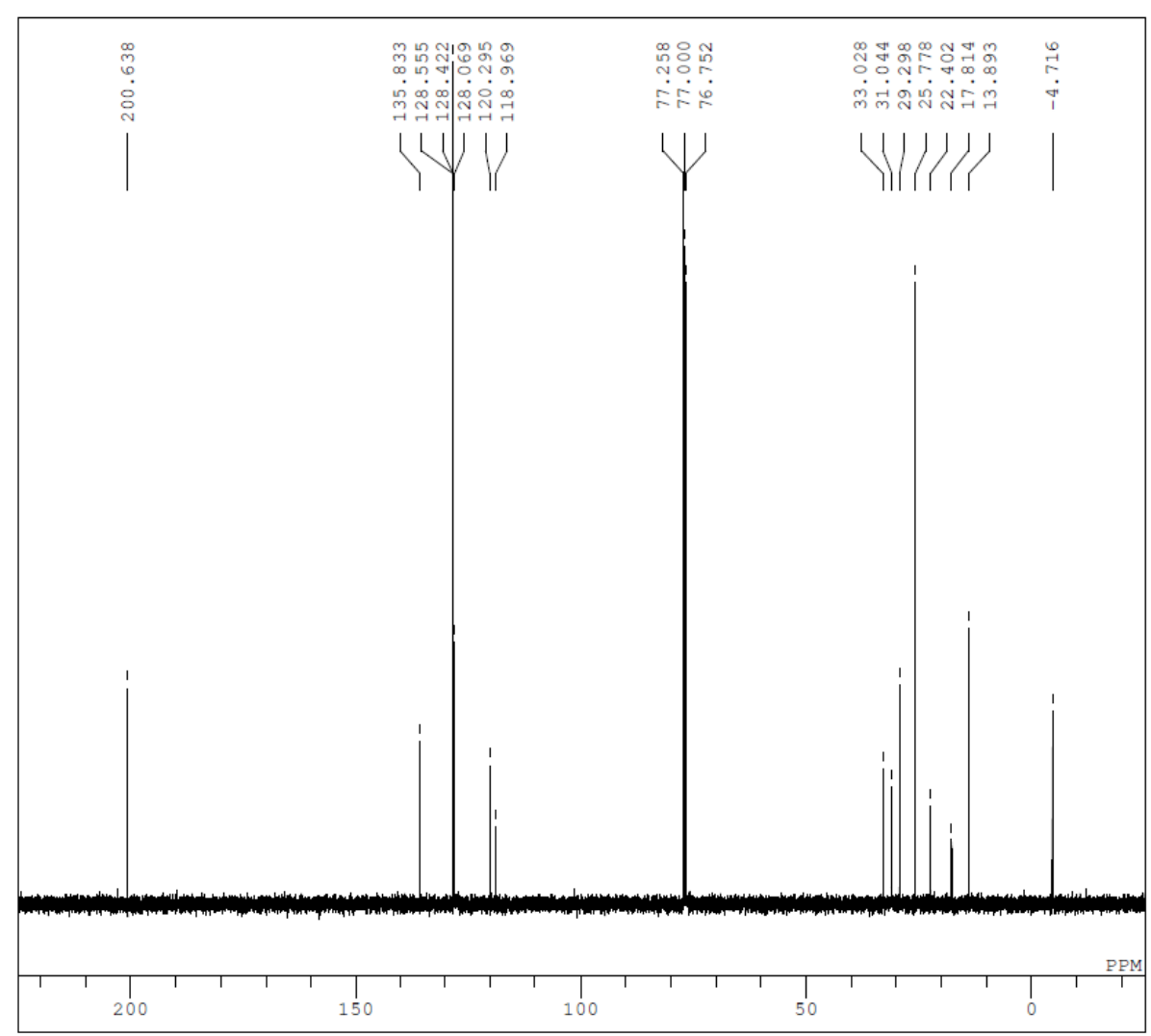

DFILE _ky_01_066_2_Carbon-1-1.als

COMNT Ms_allenamide

DATIM 2013-10-16 01:57:28

OBNUC $13 \mathrm{C}$

$\begin{array}{ll}\text { EXMOD } & \text { carbon. jXP } \\ \text { OBFRQ } & 125.77 \mathrm{MHz}\end{array}$

$\begin{array}{rr}\text { OBFRQ } & 125.77 \mathrm{MHz} \\ \text { OBSET } & 7.87 \mathrm{KHz}\end{array}$

$\begin{array}{ll}\text { OBSET } & 7.87 \mathrm{KHz} \\ \text { OBEIN } & 4.21 \mathrm{~Hz}\end{array}$

PBFIN $\quad 2.21 \mathrm{HZ}$

FREQU $\quad 31446.54 \mathrm{~Hz}$

SCANS 149

ACQTM $0.8336 \mathrm{sec}$

PD $2.0000 \mathrm{sec}$

IRNUC 1H

CTEMP 18.4

$\begin{array}{lll}\text { SLVNT } & \text { CDCL3 } 37.00 \mathrm{pPm} \\ \text { EXREF } & & \end{array}$

$\begin{array}{lr}\text { EXREF } & 77.00 \mathrm{ppm} \\ \text { BF } & 0.10 \mathrm{~Hz} \\ \text { RGAIN } & 56\end{array}$ 


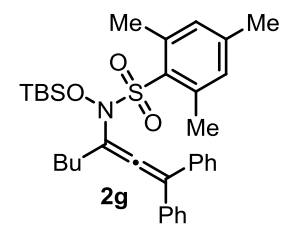

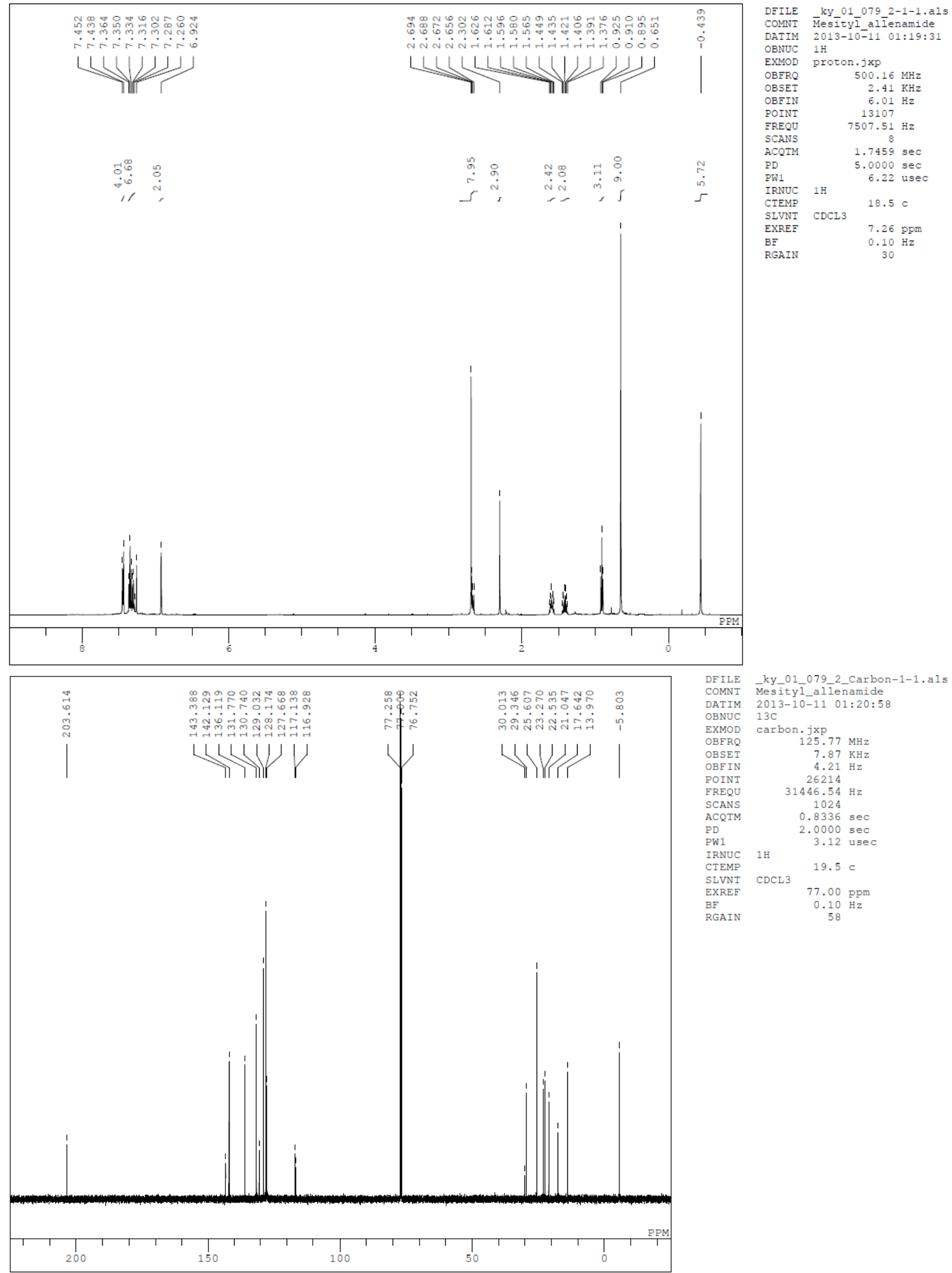


TBSO,

$\underbrace{P h}_{2 h}$
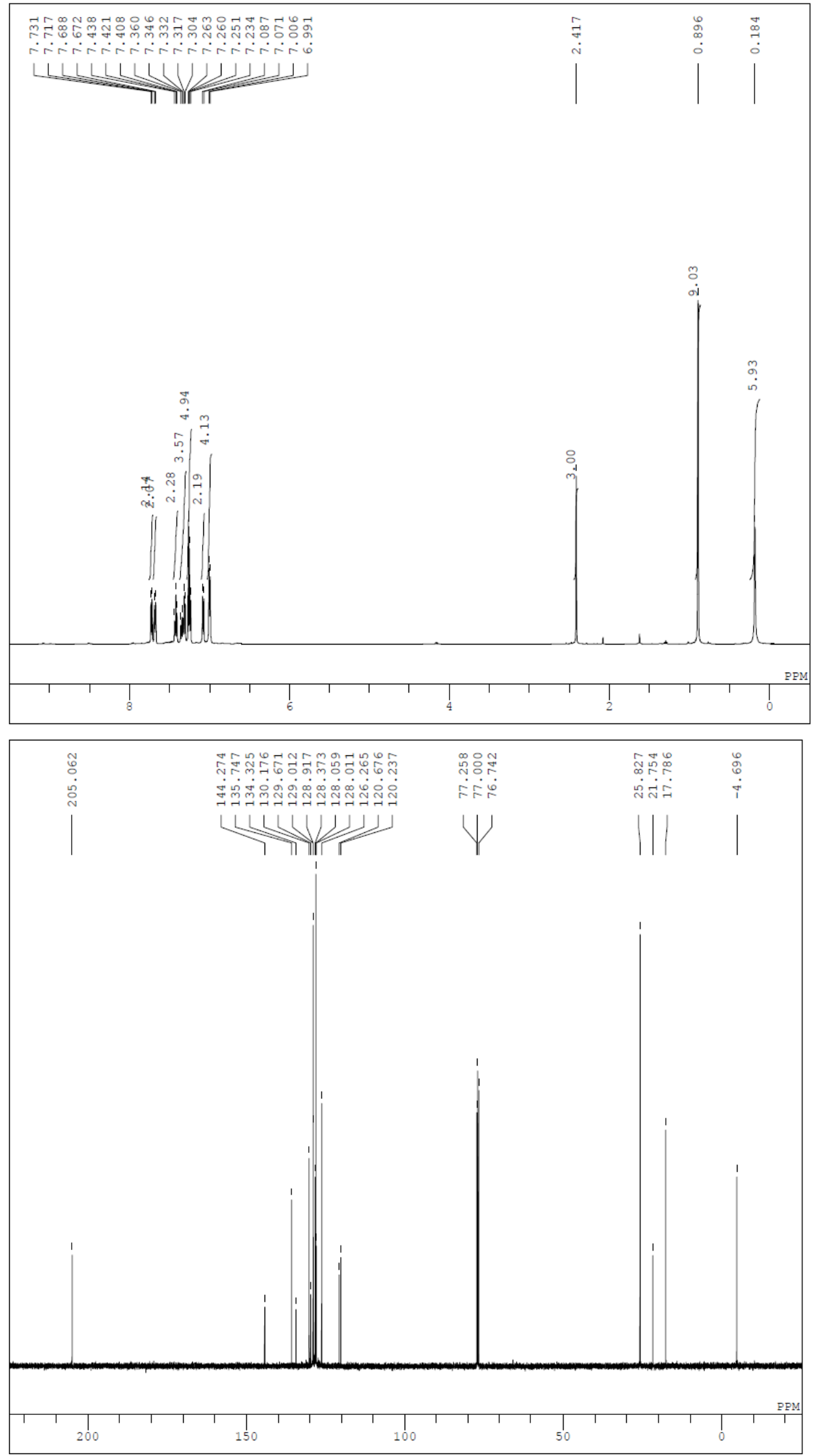

DFILE $\quad-$ ymm-05_053_4_ $13 \mathrm{C}-1-1 . j \mathrm{df}$

DATIM 2013-09-04 11:59:58

OBNUC $13 \mathrm{C}$

EXMOD carbon, jXP

OBFRO $125.77 \mathrm{MHz}$

OBSET $\quad 7.87 \mathrm{KHz}$

OBEIN $\quad 4.21 \mathrm{~Hz}$ POINT 32767

FREQU $39308.18 \mathrm{~Hz}$

SCANS $\quad 361$

ACQTM $\quad 0.8336 \mathrm{sec}$

$\begin{array}{lr}\text { PD } & 2.0000 \mathrm{sec} \\ \text { PW1 } & 3.12 \text { usec }\end{array}$

IRNUC $1 \mathrm{H}$

CTEMP $18.0 \mathrm{C}$

SIVNT CDCL3

EXRE

RGA

$77.00 \mathrm{ppm}$

$.00 \mathrm{ppm}$
$.10 \mathrm{~Hz}$
56

OBFRQ $\quad 500.16 \mathrm{MH}$

$6.01 \mathrm{~Hz}$

$507.51 \mathrm{~Hz}$

$7459 \mathrm{sec}$

22 usec

.7

$26 \mathrm{ppm}$ 
<smiles>CC(C)(C)ON([13CH3])C(=C=C(c1ccccc1)c1ccccc1)c1ccc(Br)cc1</smiles>

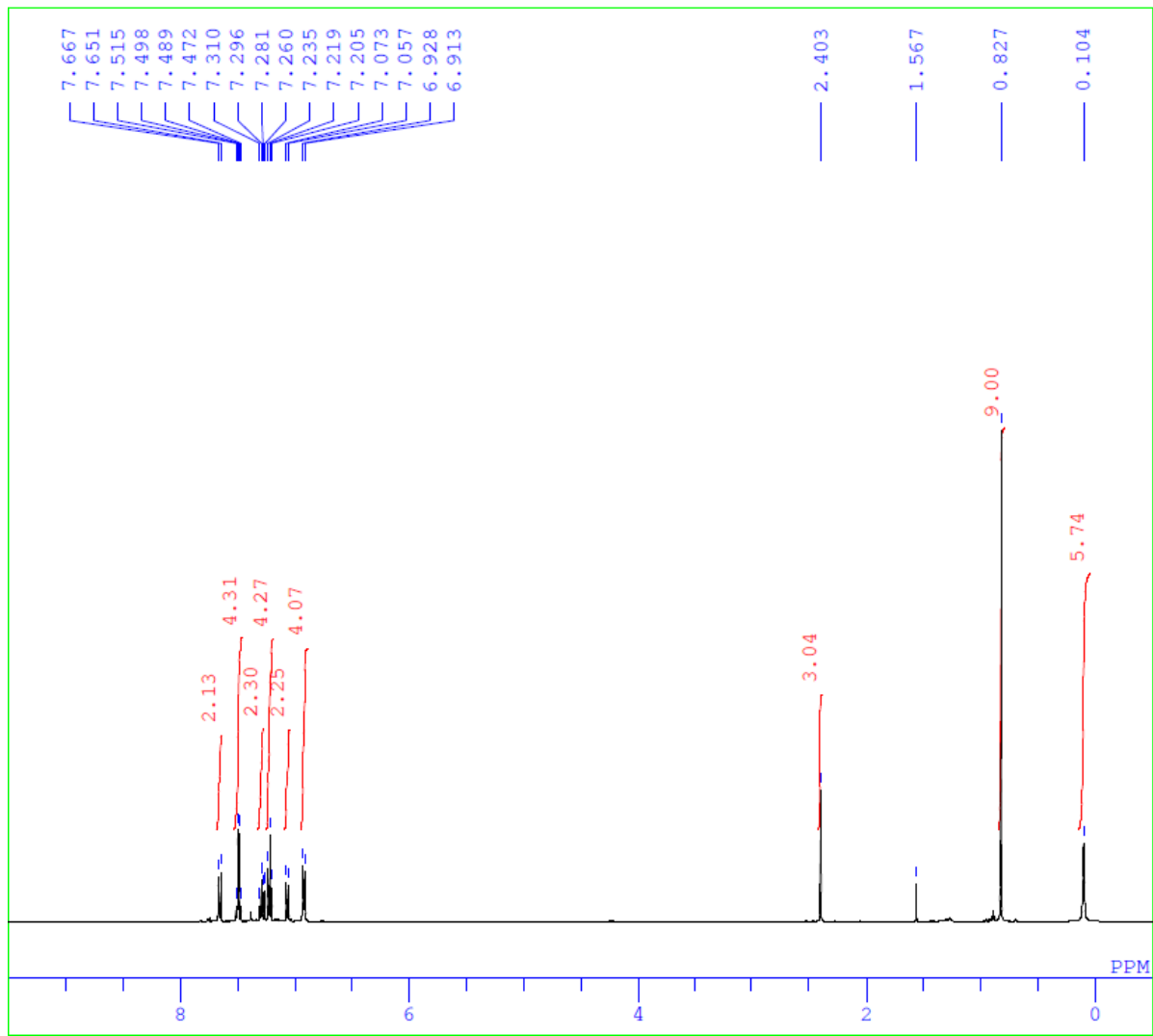

DFILE
COMNT
IH

DATIM 2013-12-20 14:51:53

OBNUC $1 \mathrm{H}$

EXMOD proton.jxp

OBFRQ $500.16 \mathrm{MHz}$

$\begin{array}{rr}\text { OBFRQ } & 5.16 \mathrm{MHz} \\ \text { OBSET } & 2.41 \mathrm{KHz}\end{array}$

OBFIN $\quad 6.01 \mathrm{~Hz}$

POINT 13107

FREQU $\quad 7507.51 \mathrm{~Hz}$

$\begin{array}{lr}\text { FREQU } & 7507.51 \mathrm{~Hz} \\ \text { SCANS } & 8\end{array}$

$\begin{array}{lr}8 \\ \text { SCANS } & 8 \\ \text { ACQTM } & 1.7459 \mathrm{sec}\end{array}$

$\begin{array}{ll}1.7459 & \mathrm{sec} \\ \mathrm{PD} & 5.0000 \mathrm{sec}\end{array}$

PW1 6.22 usec

IRNUC $1 \mathrm{H}$

CTEMP

$19.1 \mathrm{C}$

EXREF

$\mathrm{BF}$

RGAIN

$7.26 \mathrm{ppm}$

$0.10 \mathrm{~Hz}$$$
\text { (n) }
$$ 


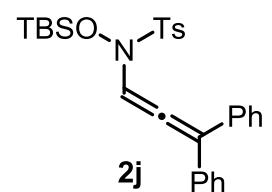
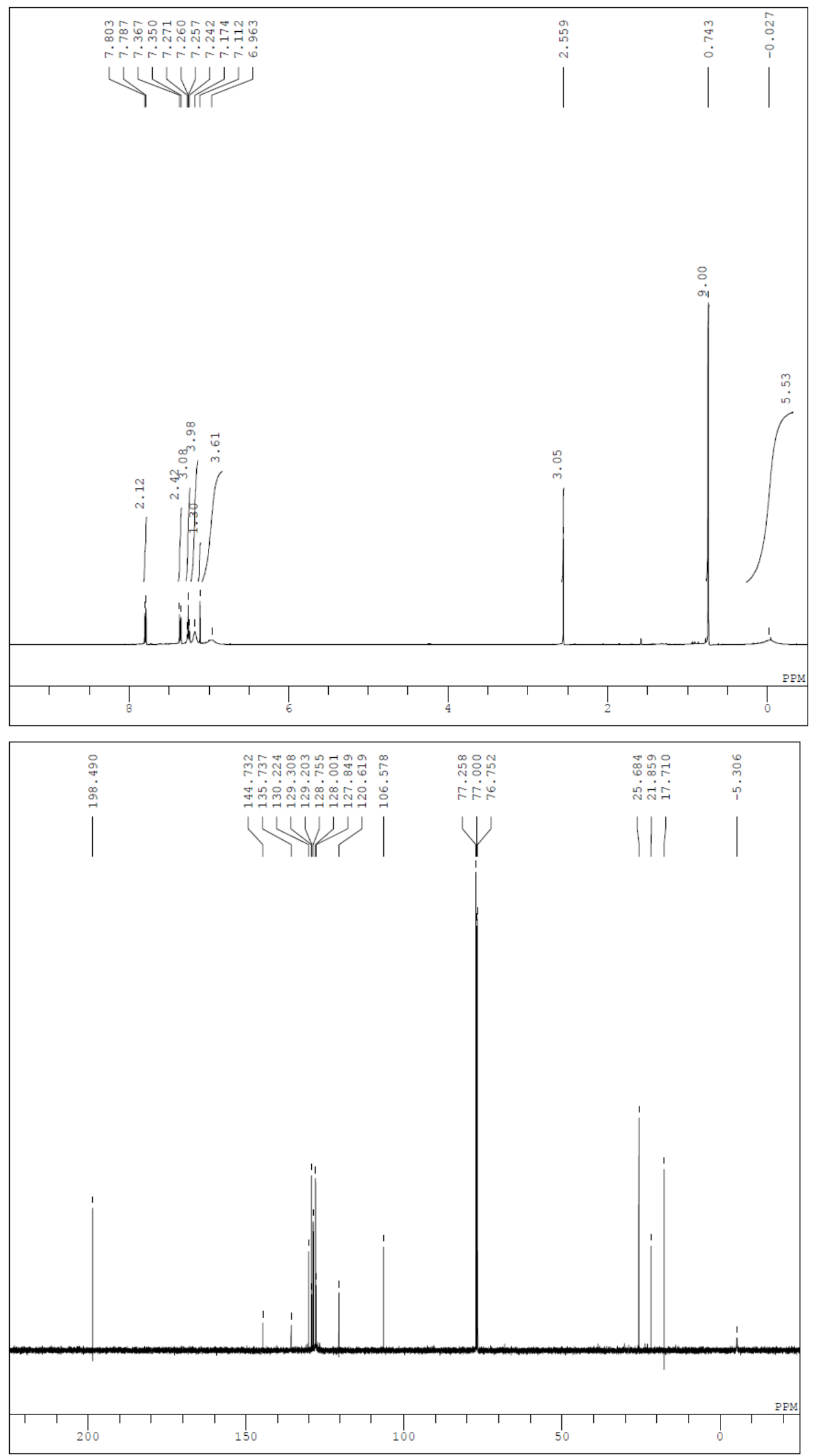

DFILE -ym_05_129_1_13C-1-1.als

DATIM 2013-12-25 18:03:08

OBNUC $13 \mathrm{C}$

EXMOD carbon.jxp

OBFRO $125.77 \mathrm{MHz}$

OBSET $\quad 7.87 \mathrm{KHz}$

OBFIN $\quad 4.21 \mathrm{~Hz}$ POINT 32767

FREQU $\quad 39308.18 \mathrm{~Hz}$

SCANS $\quad 512$

ACQTM $\quad 0.8336 \mathrm{sec}$

$\begin{array}{lr}\text { PD } & 2.0000 \text { sec } \\ \text { PW1 } & 3.12 \text { usec }\end{array}$

IRNUC $1 \mathrm{H}$

CTEMP $18.8 \mathrm{C}$

SLVNT CDCL3

EXREF

RGA

$77.00 \mathrm{ppr}$

$0.10 \mathrm{~Hz}$ 
<smiles>CCCCCCC(=C=C(c1ccccc1)c1ccccc1)N([18OH])c1ccccc1</smiles>

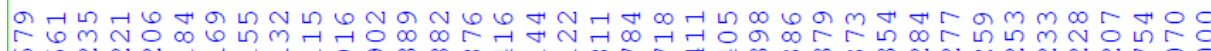

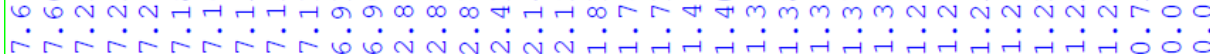

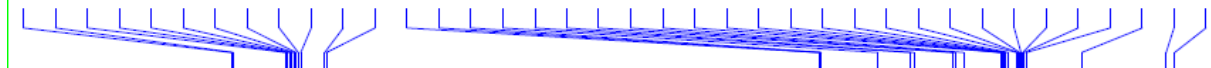

DFILE

COMNT

DATIM

OBNUC

EXMOD

OBFRQ

OBSET

OBEIN

POINT

FREQU

SCANS

ACQTM

$\mathrm{PD}$

PW1

IRNUC

CTEMP

SLVNT

EXREF

$\mathrm{BF}$

RGAIN

CDCL

inge

2014-0 $8-19$ 20:31:11

proton.jxp

$500.16 \mathrm{MHz}$

$2.41 \mathrm{KHz}$

$6.01 \mathrm{~Hz}$

13107

$7507.51 \mathrm{~Hz}$ 8

1. 7459 sec

$5.0000 \mathrm{sec}$

6.22 usec

$20.6 \mathrm{c}$

$0.00 \mathrm{ppm}$

$0.10 \mathrm{~Hz}$ 24
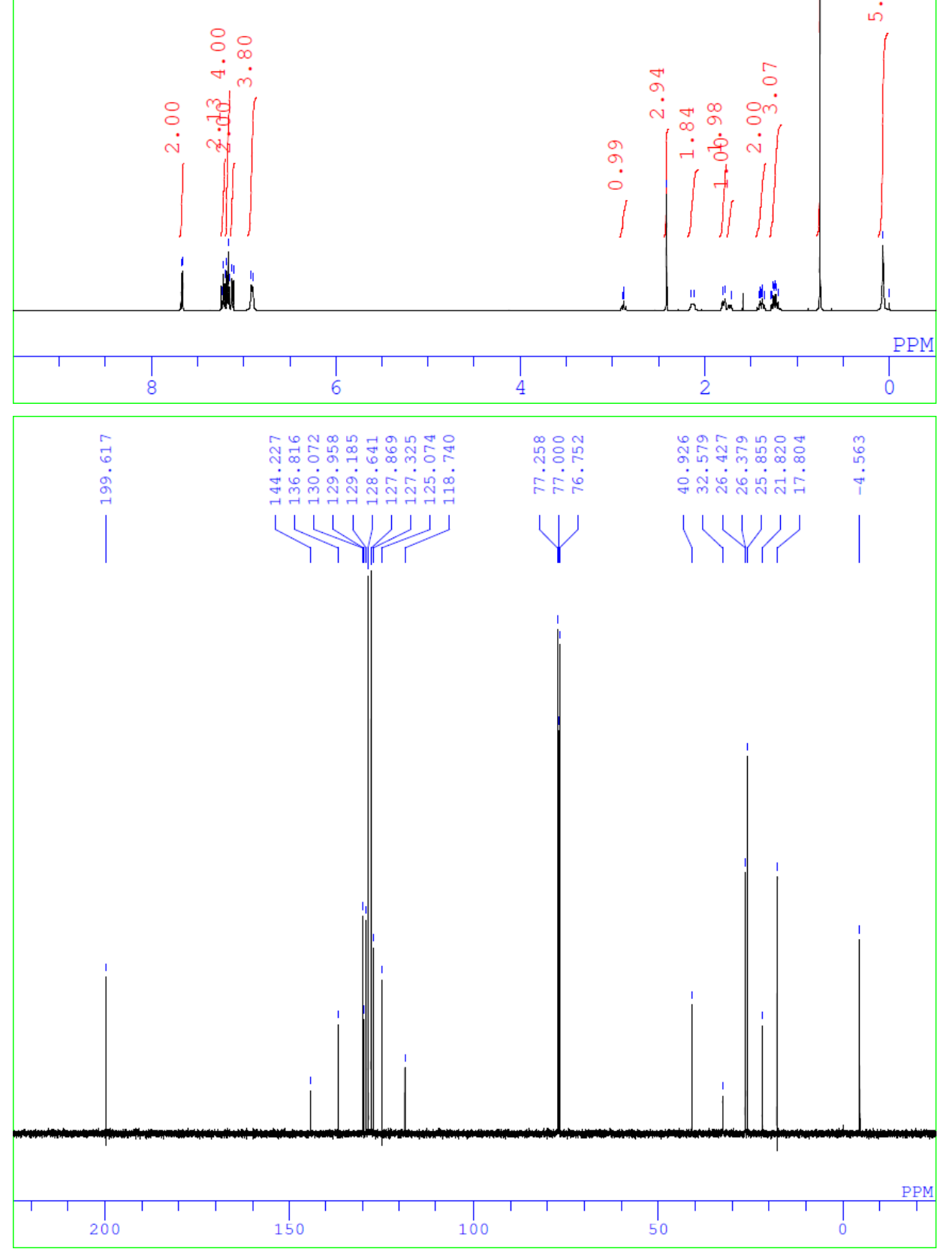

DFILE

COMNT

20:34:49

OBNUC $13 \mathrm{C}$

MOD carbon.jxp

OBERQ $\quad 125.77 \mathrm{MHz}$

OBSET

OBF IN

POINT

FREQU

SCANS

ACQTM

$\mathrm{PD}$

PW1

IRNUC

CTEMP

SLVNT

EXREF

RGAIN

$7.87 \mathrm{KHz}$

$4.21 \mathrm{~Hz}$

26214

$31446.54 \mathrm{~Hz}$

542

$0.8336 \mathrm{sec}$

$2.0000 \mathrm{sec}$

$1 \mathrm{H}$

CDCL 3

$20.7 \mathrm{C}$

$77.00 \mathrm{ppm}$

$0.10 \mathrm{~Hz}$ 

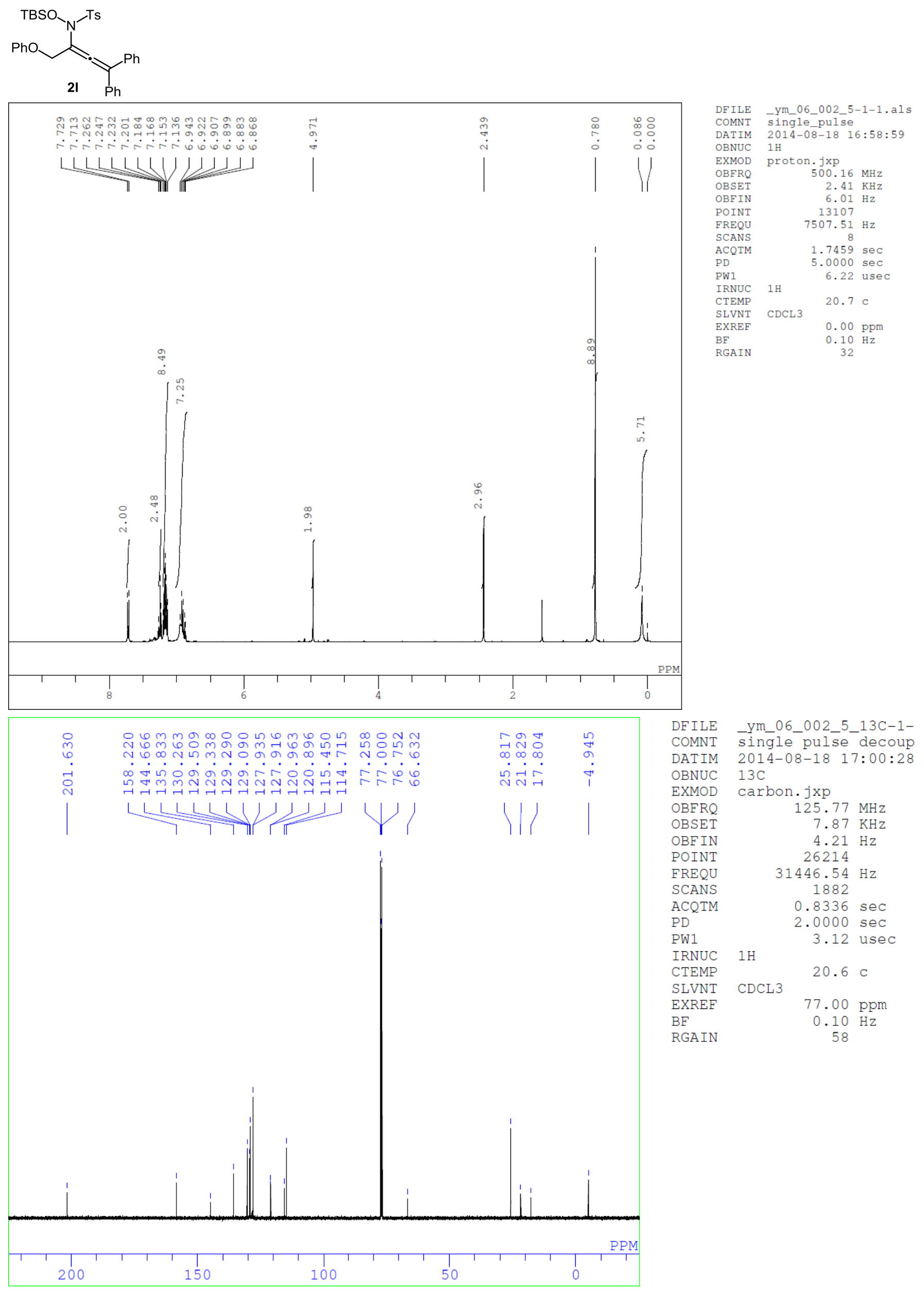

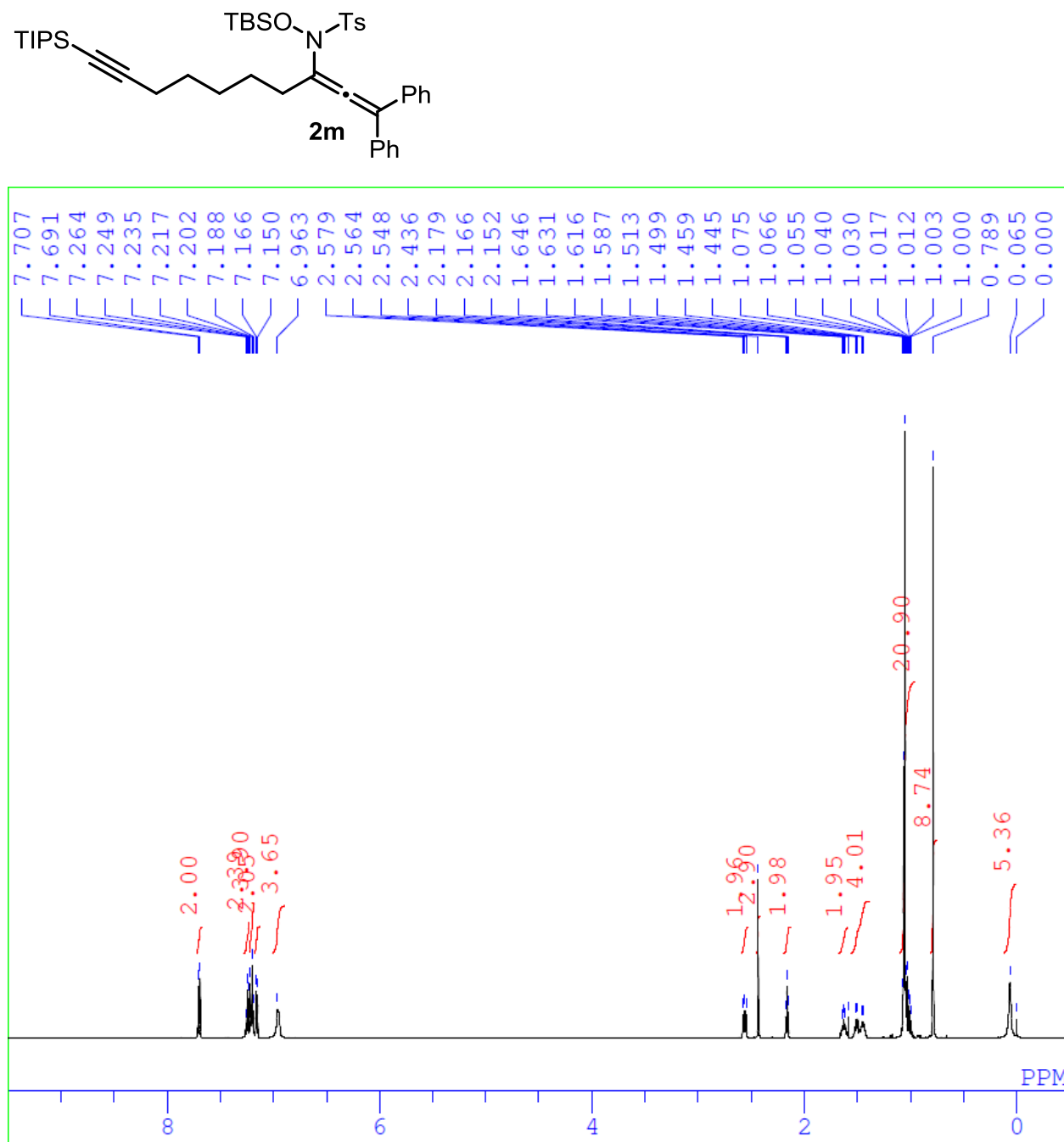

DE ILE

COMNT

DATIM

OBNUC

EXMOD

OBFRQ

OBSET

OBEIN

POINT

FREQU

SCANS

ACQTM

$\mathrm{PD}$

PW1

IRNUC

CTEMP

SLVNT

EXREF

$\mathrm{BE}$

RGAIN ym 06017 2-1-1.a1 single_pulse

2014-09-04 17:12:25 $1 \mathrm{H}$

proton.jxp

$500.16 \mathrm{MHz}$ 2. $41 \mathrm{KHz}$

$6.01 \mathrm{~Hz}$

13107

$7507.51 \mathrm{~Hz}$

8
$1.7459 \mathrm{sec}$

5.0000 sec

6.22 usec

$1 \mathrm{H}$

CDCL 3

$18.9 \mathrm{C}$

$0.00 \mathrm{ppm}$

$0.10 \mathrm{~Hz}$

22

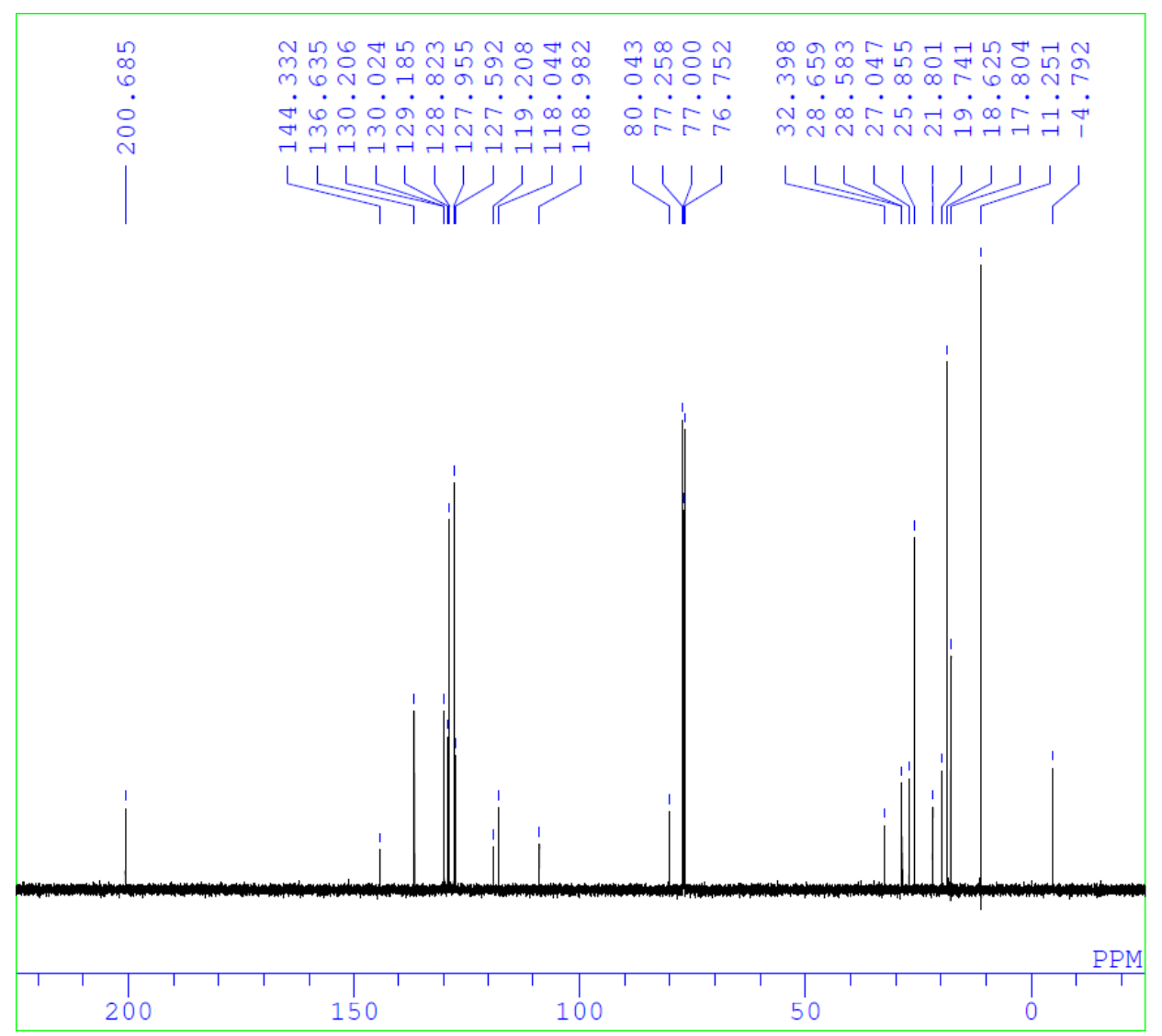

DEILE

COMNT

DATIM

OBNUC

EXMOD

OBERO

OBSET

OBEIN

POINT

FREQU

SCANS

ACQTM

$\mathrm{PD}$

PW1

IRNUC

CTEMP

SLVNT

EXREF

$\mathrm{BE}$

RGAIN ym $06 \quad 017 \quad 2 \quad 13 \mathrm{C}-1$ single pulse decoup 2014-09-04 17:13:55 $13 \mathrm{C}$

carbon. jxp

$125.77 \mathrm{MHz}$

$7.87 \mathrm{KHz}$

$4.21 \mathrm{~Hz}$

26214

$31446.54 \mathrm{~Hz}$

202

0.8336 sec

2.0000 sec

$1 \mathrm{H}$

3.12 usec

CDCL 3

$19.1 \mathrm{C}$

$77.00 \mathrm{ppm}$

$0.10 \mathrm{~Hz}$

58 

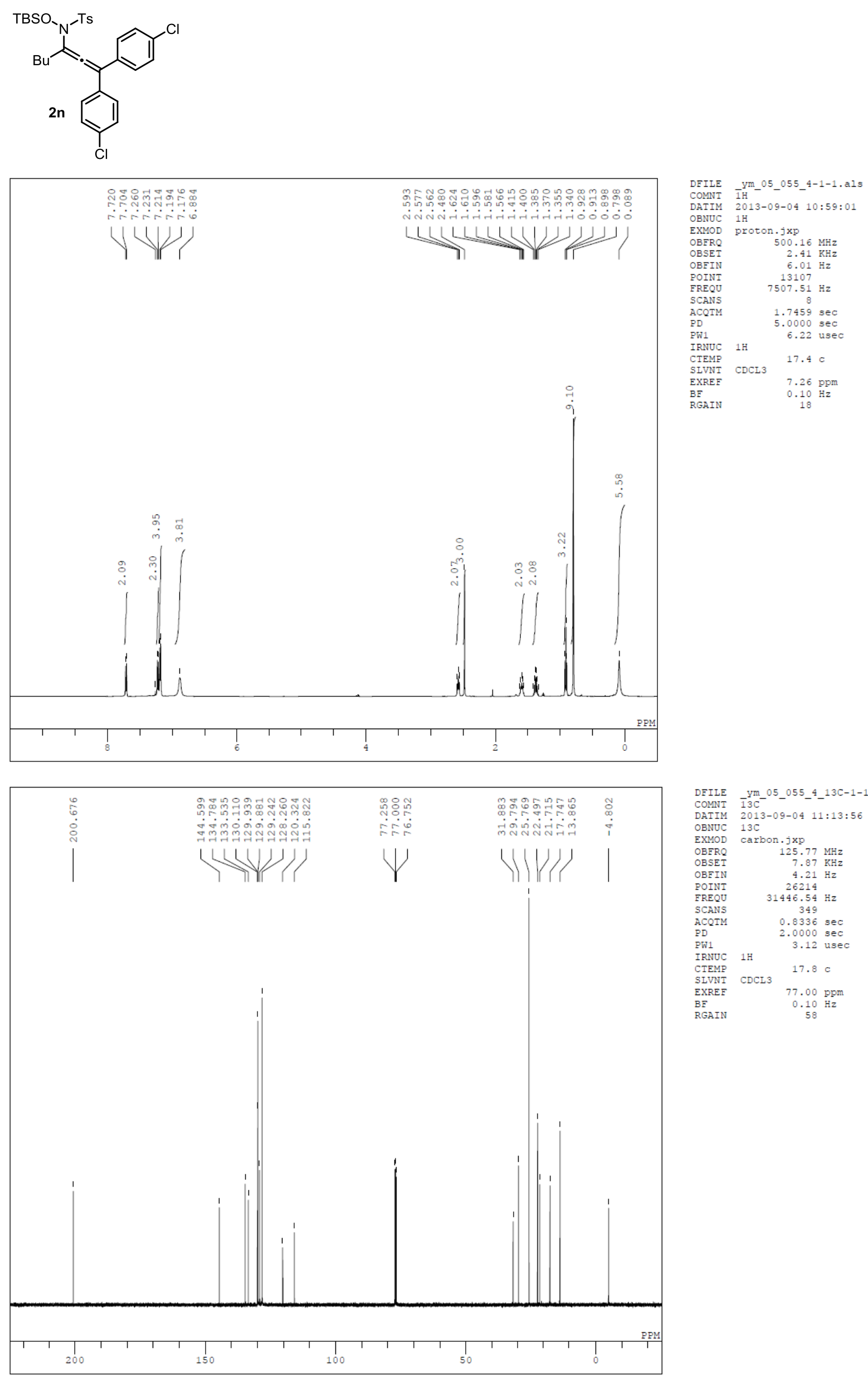

DFILE_Ym_05_055_4_13C-1-1.al

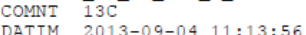

OBNUC $13 \mathrm{C}$

EXMOD carbon.jXP

$\begin{array}{lr}\text { OBFRQ } & 125.77 \mathrm{MHz} \\ \text { OBSET } & 7.87 \mathrm{KHH}\end{array}$

$\begin{array}{ll}\text { OBSET } & 7.87 \mathrm{KHz} \\ \text { OBFIN } & 4.21 \mathrm{~Hz}\end{array}$

OBFIN $\quad 4.21 \mathrm{~Hz}$

FREQU $31446.54 \mathrm{~Hz}$

$\begin{array}{ll}\text { SCANS } & 349 \\ \text { ACQTM } & 0.8336 \mathrm{sec}\end{array}$

$\begin{array}{ll}\text { ACQTM } & 0.8336 \mathrm{sec} \\ \text { PD } & 2.0000 \mathrm{sec}\end{array}$

PW1 3.12 use

CTEMP

SLVNT CDCL3

EXREF $2 D C L 377.00 \mathrm{ppm}$

$\begin{array}{lc}\text { BF } & 0.10 \mathrm{~Hz} \\ \text { RGAIN } & 58\end{array}$ 

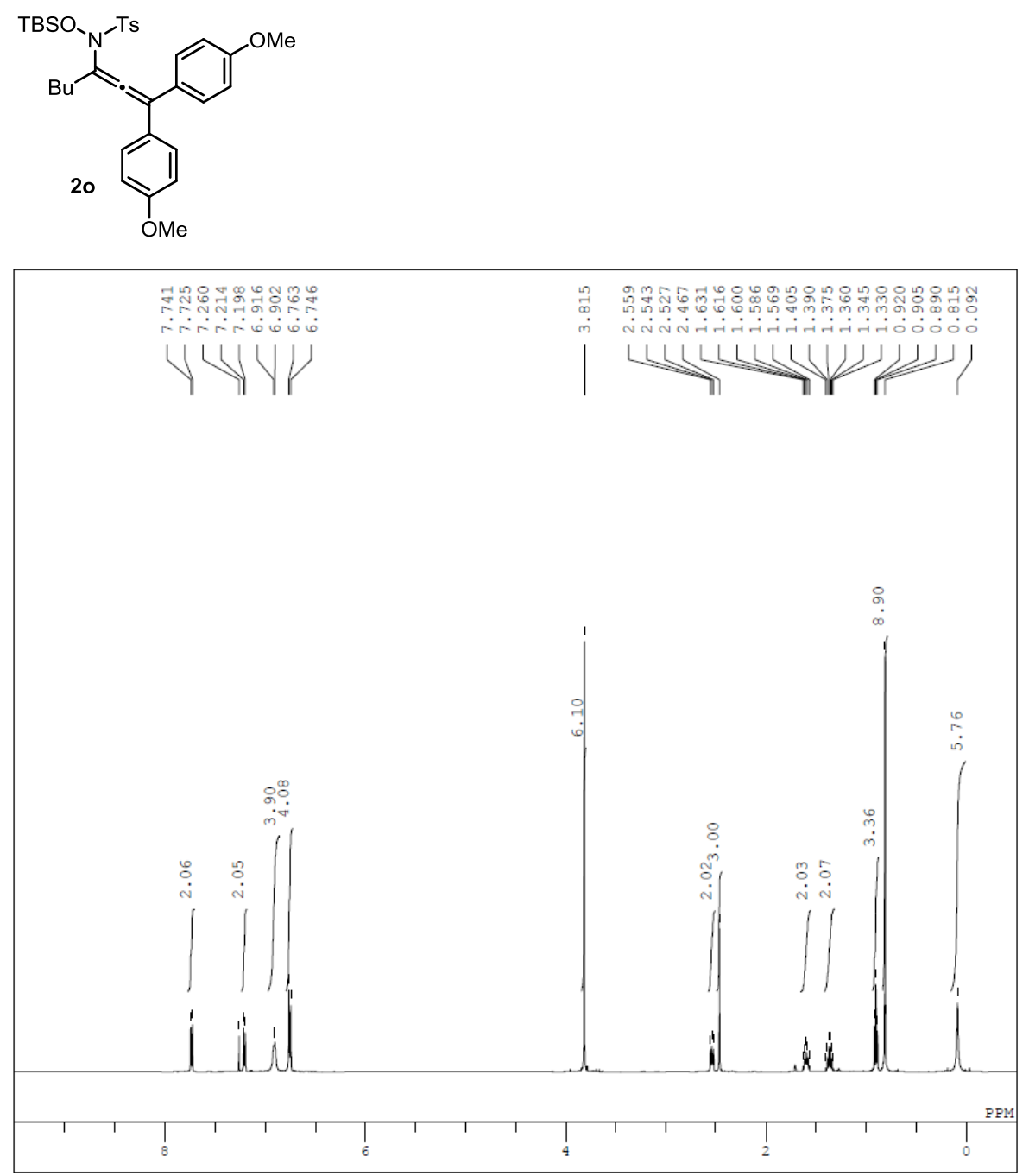

DFILE YYM_05_056_2-1-1.a1s

DATIM 2013-09-03 17:44:51

BNUC $1 \mathrm{H}$

DXMOD proton.jxp

$\begin{array}{lr}\text { OBRRQ } & 500.16 \mathrm{MH} \\ \text { OBSET } & 2.41 \mathrm{KH}\end{array}$

OBEIN $\quad 6.01 \mathrm{~Hz}$

$\begin{array}{lr}\text { POINT } & 13107 \\ \text { FREQU } & 7507.51 \mathrm{H}\end{array}$

SCANS 8

ACQTM $\quad 1.7459 \mathrm{sec}$

$\begin{array}{lr}\text { PD } & 5.0000 \text { sec } \\ \text { PW1 } & 6.22 \text { usec }\end{array}$

CTEMP $1 \mathrm{H}$ 18.20

SIVNT CDCL3 3

EXREF

BF

$7.26 \mathrm{ppm}$
$0.10 \mathrm{~Hz}$

TEAIN

DFILE YM_05_056_2_13C-1-1.jde

COMNT $13 \mathrm{C}^{-}-{ }^{-}-1 \mathrm{C}$

DATIM $2013-09-03 \quad 17: 46: 17$

OBNUC $13 \mathrm{C}$

EXMOD carbon.jXP

$\begin{array}{lr}\text { OBRRQ } & 125.77 \mathrm{MHz} \\ \text { OBSET } & 7.87 \mathrm{KHz}\end{array}$

OBFIN $4.21 \mathrm{~Hz}$

POINT 32767

FREQU $39308.18 \mathrm{~Hz}$

$\begin{array}{ll}\text { SCANS } & 1024 \\ \text { ACQTM } & 0.8336 \mathrm{sec}\end{array}$

$\begin{array}{ll}\text { ACQTM } & 0.8336 \mathrm{sec} \\ \text { PD } & 2.0000 \mathrm{sec}\end{array}$

PW1 3.12 usec

CTEMP 19.2 O

SLVNT CDCL3

EXREF CDCL3 $77.00 \mathrm{ppm}$

$\begin{array}{lc}\text { BE } & 0.10 \mathrm{~Hz} \\ \text { RGAIN } & 58\end{array}$

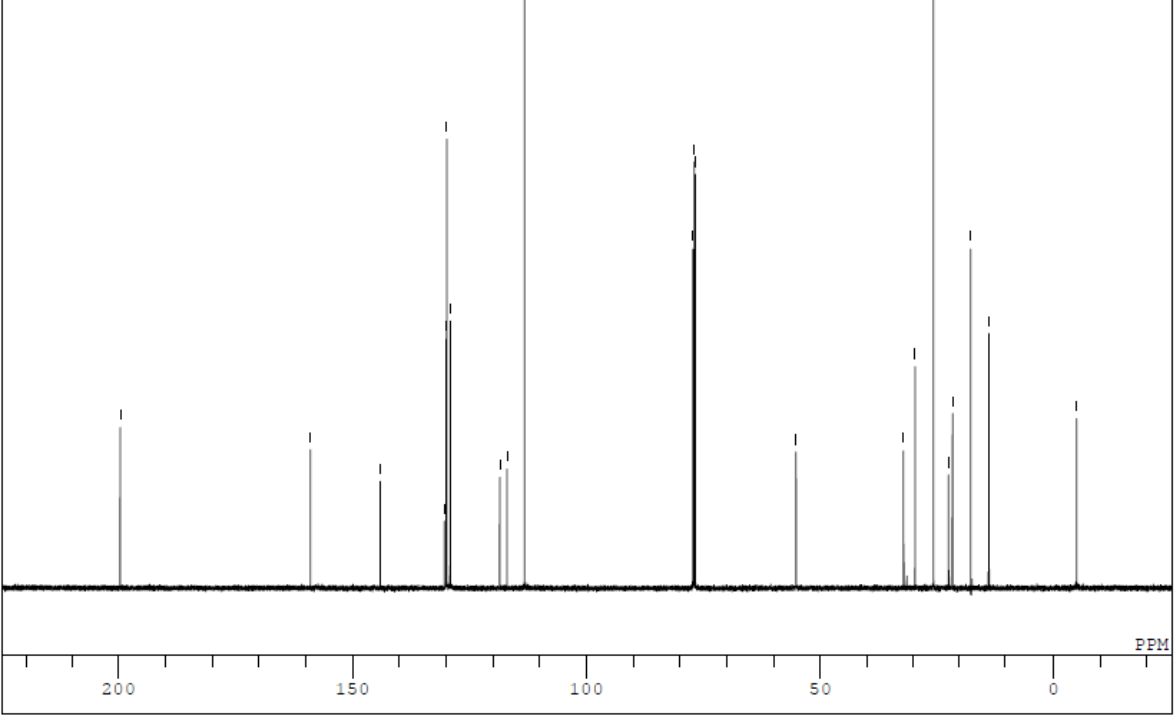



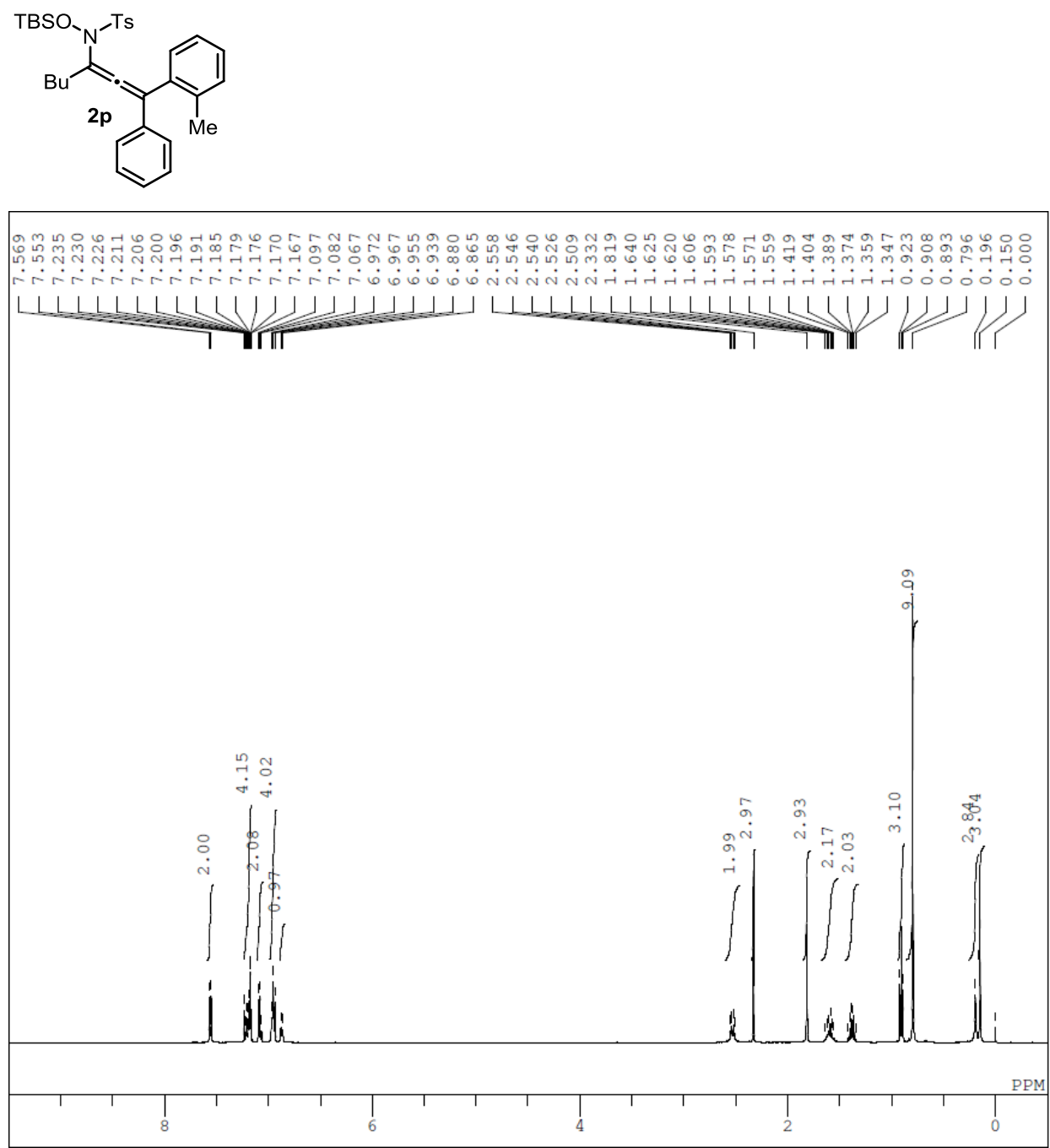

DFILE -YM_06_047_3-1-2.als

COMNT single_pulse

DATIM 2014-09-27 11:43:19

OBNUC $1 \mathrm{H}$

EXMOD proton.jxp

OBFRQ $\quad 500.16 \mathrm{MHz}$

OBSET $2.41 \mathrm{KHz}$

OBFIN $\quad 6.01 \mathrm{~Hz}$

POINT 16384

FREQU $\quad 9384.38 \mathrm{~Hz}$

SCANS 8

ACQTM $1.7459 \mathrm{sec}$

PD $\quad 5.0000 \mathrm{sec}$

IRNUC 1H $1 \mathrm{H} .22$ us

CTEMP IH $19.0 \mathrm{C}$

SLVNT CDCL 3

EXREF $0.00 \mathrm{ppm}$

BE

RGAIN

$0.10 \mathrm{~Hz}$

$0.10 \mathrm{~Hz}$
22

DFILE -ym_06_047_3_13C-1-1.als

COMNT single pulse decoupled gated

DATIM 2014-09-27 11:44:50

OBNUC 13C

EXMOD carbon.jxp

OBFRQ $\quad 125.77 \mathrm{MHz}$

$\begin{array}{lr}\text { OBSET } & 7.87 \mathrm{KHz}\end{array}$

OBFIN $4.21 \mathrm{~Hz}$

POINT 26214

FREQU $31446.54 \mathrm{~Hz}$

SCANS 256

$\begin{array}{lr}\text { SCANS } & 256 \\ \text { ACQTM } & 0.8336 \mathrm{sec}\end{array}$

$\begin{array}{ll}A C Q T M & 0.8336 \\ \text { PD } & 2.0000 \mathrm{sec}\end{array}$

$\begin{array}{lr}\text { PD } & 2.0000 \mathrm{sec} \\ \text { PW1 } & 3.12 \text { usec }\end{array}$

IRNUC $1 \mathrm{H}$

SLVNT

EXREF
BF
RGAIN

CDCL3

$19.2 \mathrm{c}$

$77.00 \mathrm{ppm}$

$0.10 \mathrm{~Hz}$ 


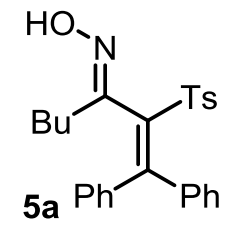

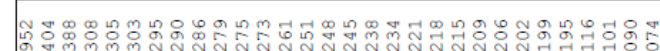

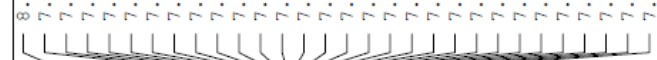

Tin

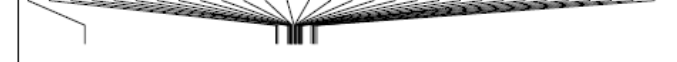

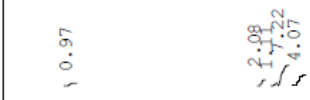
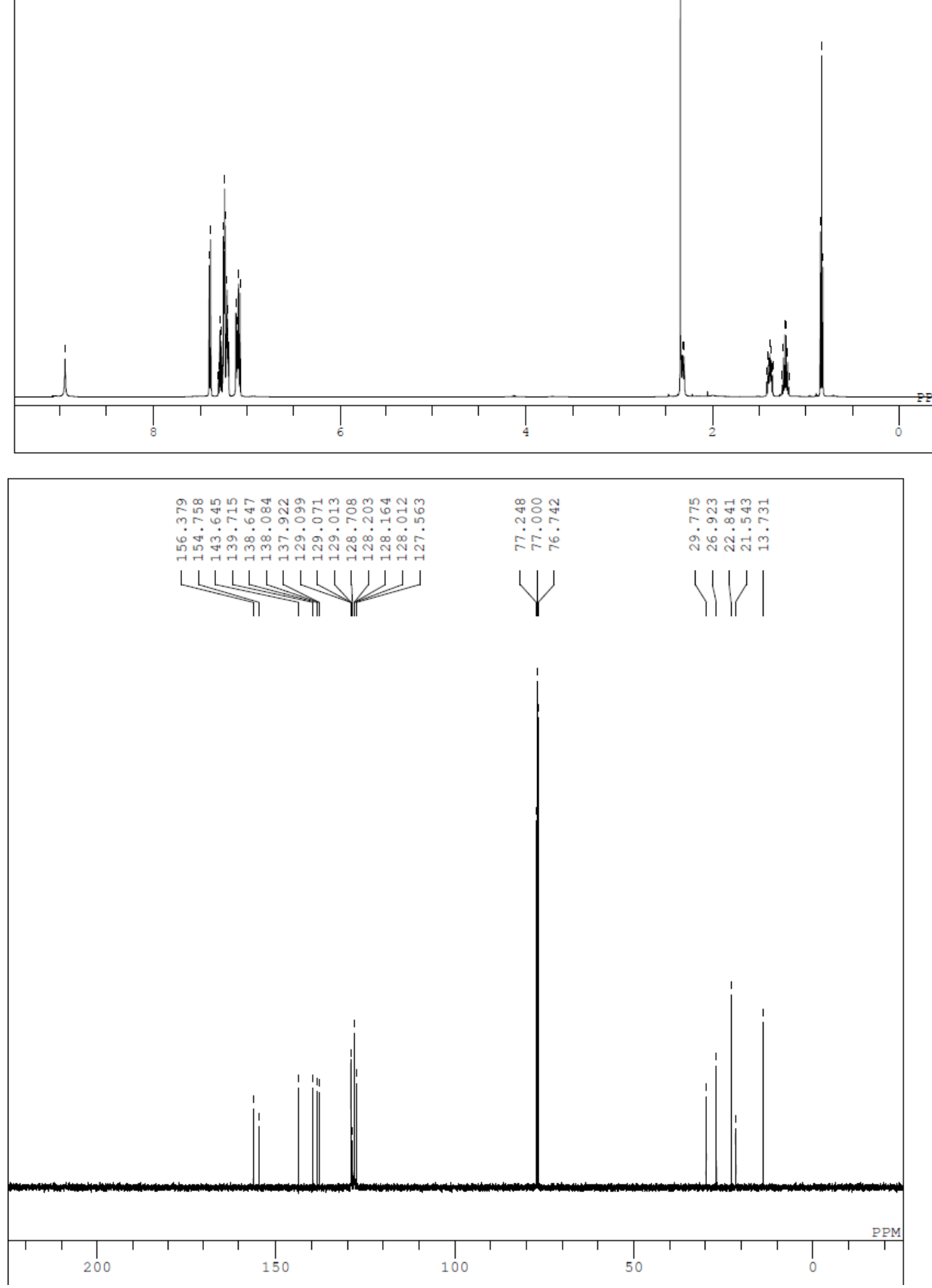

DFILE -ky_Ts_oxime_carbon-1-1.als

COMNT KY_TS

DATIM 2013-11-16 20:20:43

OBNUC $13 \mathrm{C}$

EXMOD carbon. jxp

$\begin{array}{lr}\text { OBFRQ } & 125.77 \mathrm{MHz} \\ \text { OBSET } & 7.87 \mathrm{KHz}\end{array}$ $\begin{array}{ll}\text { OBSET } & 7.87 \mathrm{KHz} \\ \text { OBFIN } & 4.21 \mathrm{~Hz}\end{array}$ $4.21 \mathrm{HZ}$
POINT FREQU $\quad 31446.54 \mathrm{~Hz}$ SCANS 422 ACQTM $\quad 0.8336 \mathrm{sec}$ PD $2.0000 \mathrm{sec}$

IRNUC $1 \mathrm{H}$

SLVNT CDCL 3

EXREF

BF

$16.1 \mathrm{c}$

CDCL3

$77.00 \mathrm{ppm}$

$0.10 \mathrm{~Hz}$ 


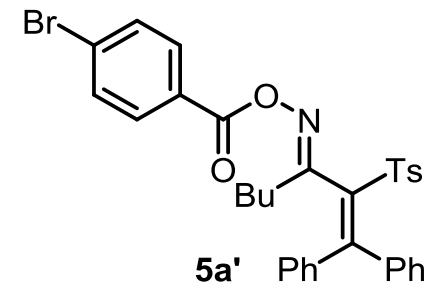

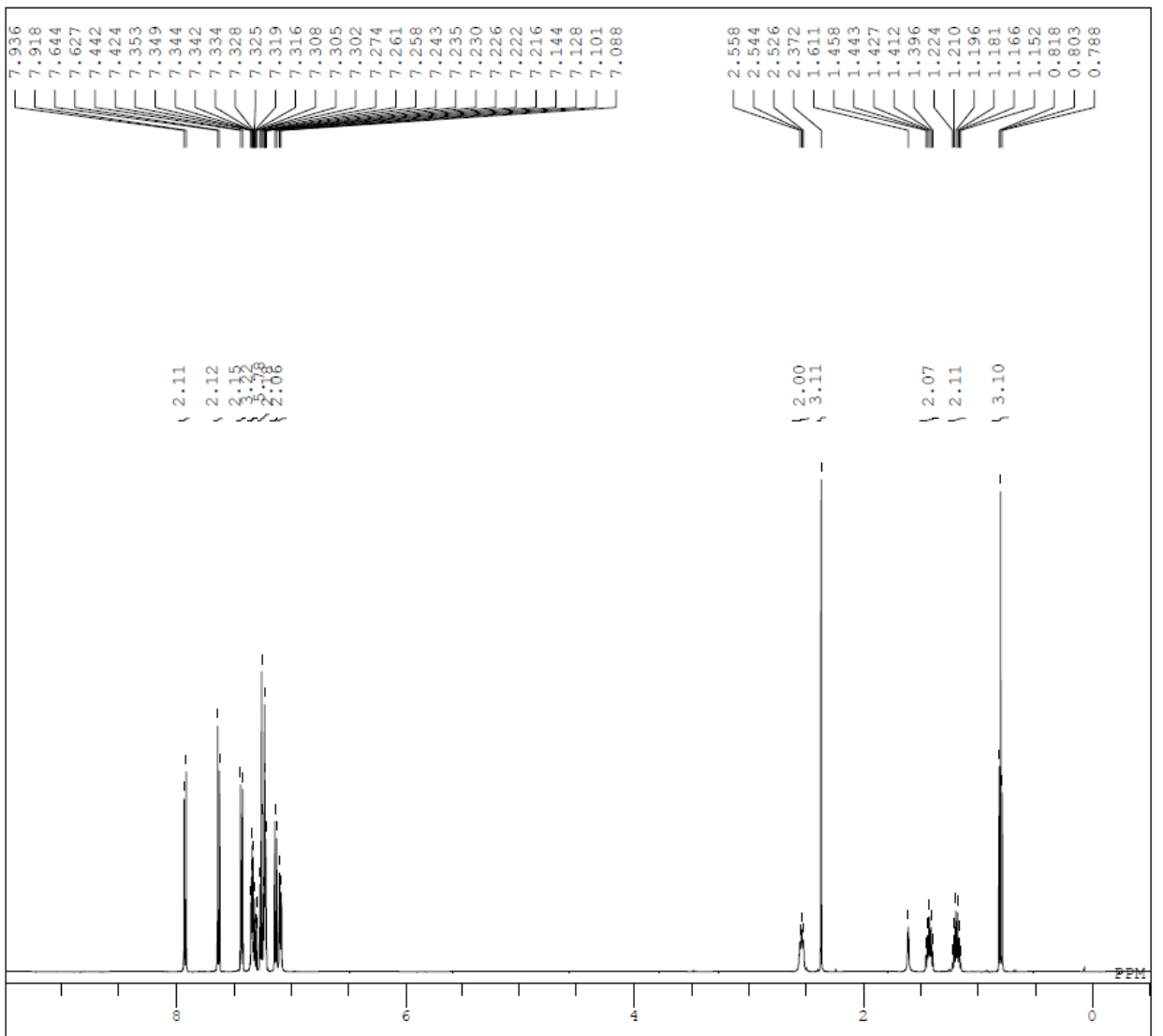

DFILE ky Ts oxime_p_BrBz-1-1.als

DAIIM $2013-11-16 \overline{2} 1: 43: 38$

OBNUC $1 \mathrm{H}$

Exoton.jxp

OBFRQ $\quad 500.16 \mathrm{MHz}$

OBSET $2.41 \mathrm{KHz}$

POINT 13107

FREQU $\quad 7507.51 \mathrm{~Hz}$

SCANS 8

PD $5.0000 \mathrm{sec}$

PW1 $\quad 6.22$ use

IRNUC
CTEMP

SIVNT CDCL3 3.0

EXREF $\quad 0.00 \mathrm{ppm}$

BF $\quad 0.10 \mathrm{~Hz}$

40

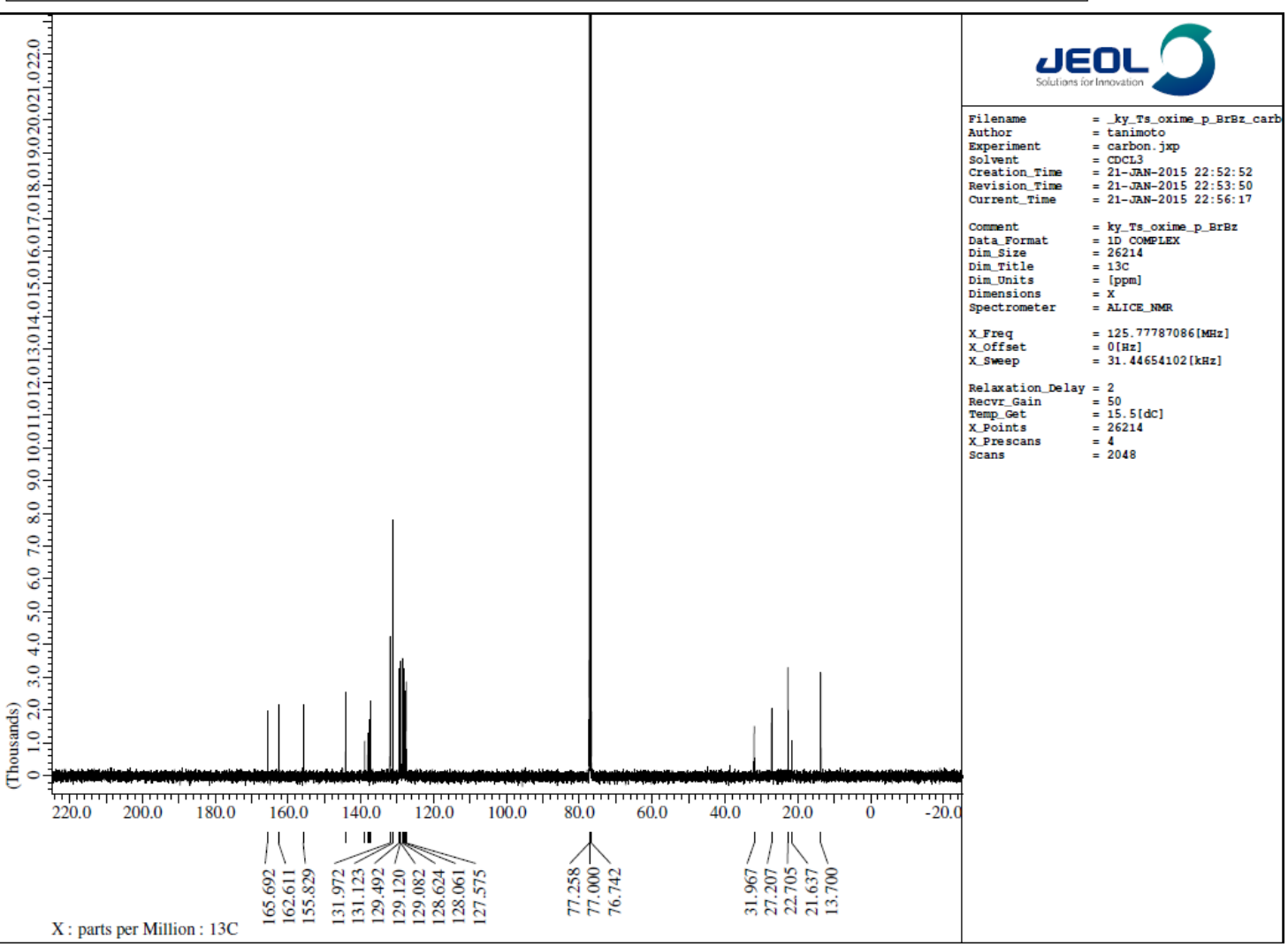



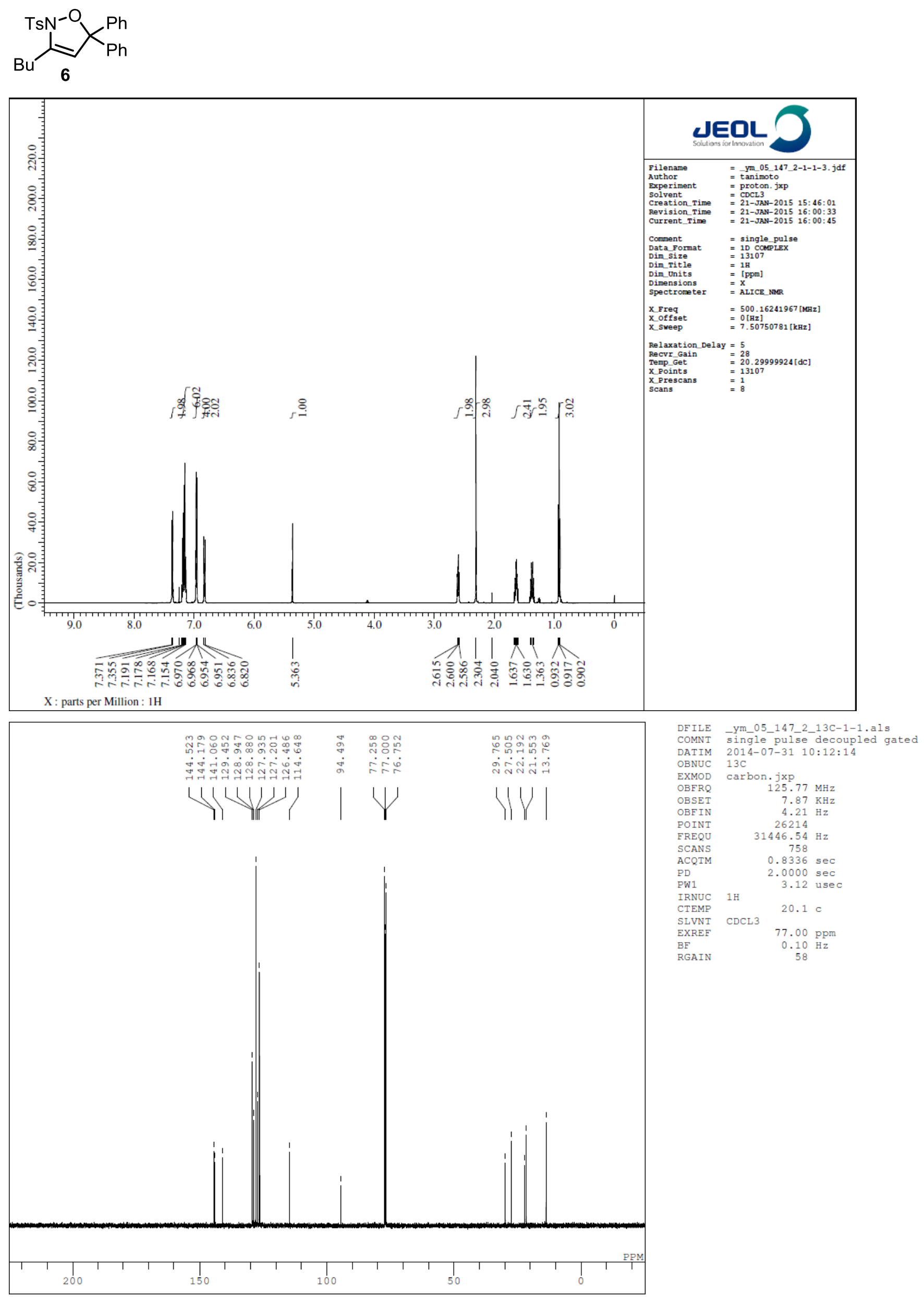
<smiles>[3H]C1=NOC(c2ccccc2)(c2ccccc2)C1</smiles>

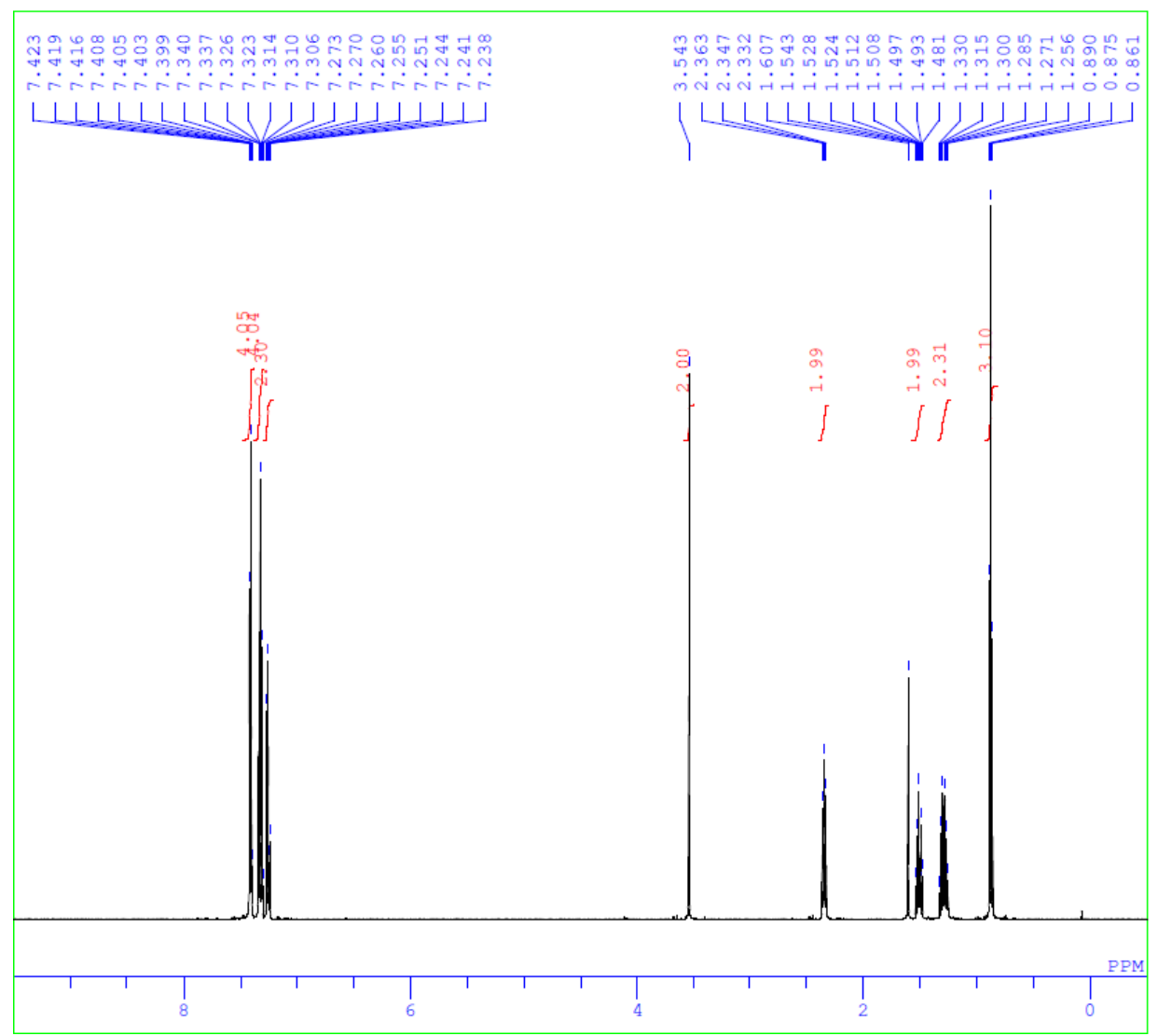

DFILE_ky_02_081_1-1-1.als

COMNT Desilylation of allenamide

OBNUC 1 (4)

EXMOD proton.jXp

OBFRQ $500.16 \mathrm{MHz}$

OBSET $\quad 2.41 \mathrm{KHZ}$

$6.01 \mathrm{~Hz}$

FREQU 7507.51 H

SCANS 8

$1.7459 \mathrm{sec}$

PD $\quad 5.0000 \mathrm{sec}$

6.22 usec

CTEMP $1 \mathrm{H} \quad 18.7$ 。

SLVNT CDCL 3

EXREF $7.26 \mathrm{pPm}$

$\begin{array}{lr}\text { EXREF } & 7.26 \mathrm{ppm} \\ \text { BF } & 0.10 \mathrm{~Hz} \\ \text { RGAIN } & 38\end{array}$

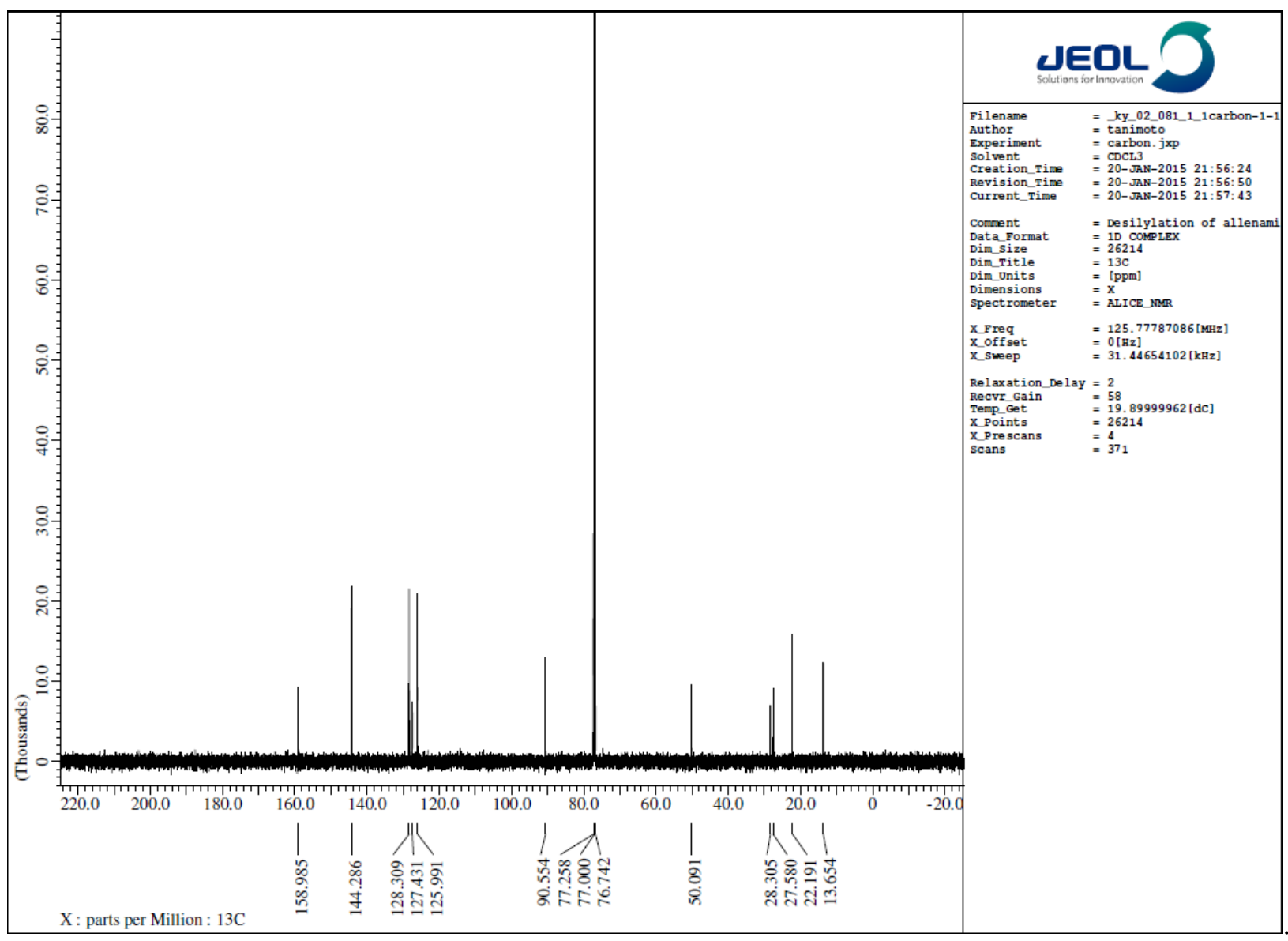



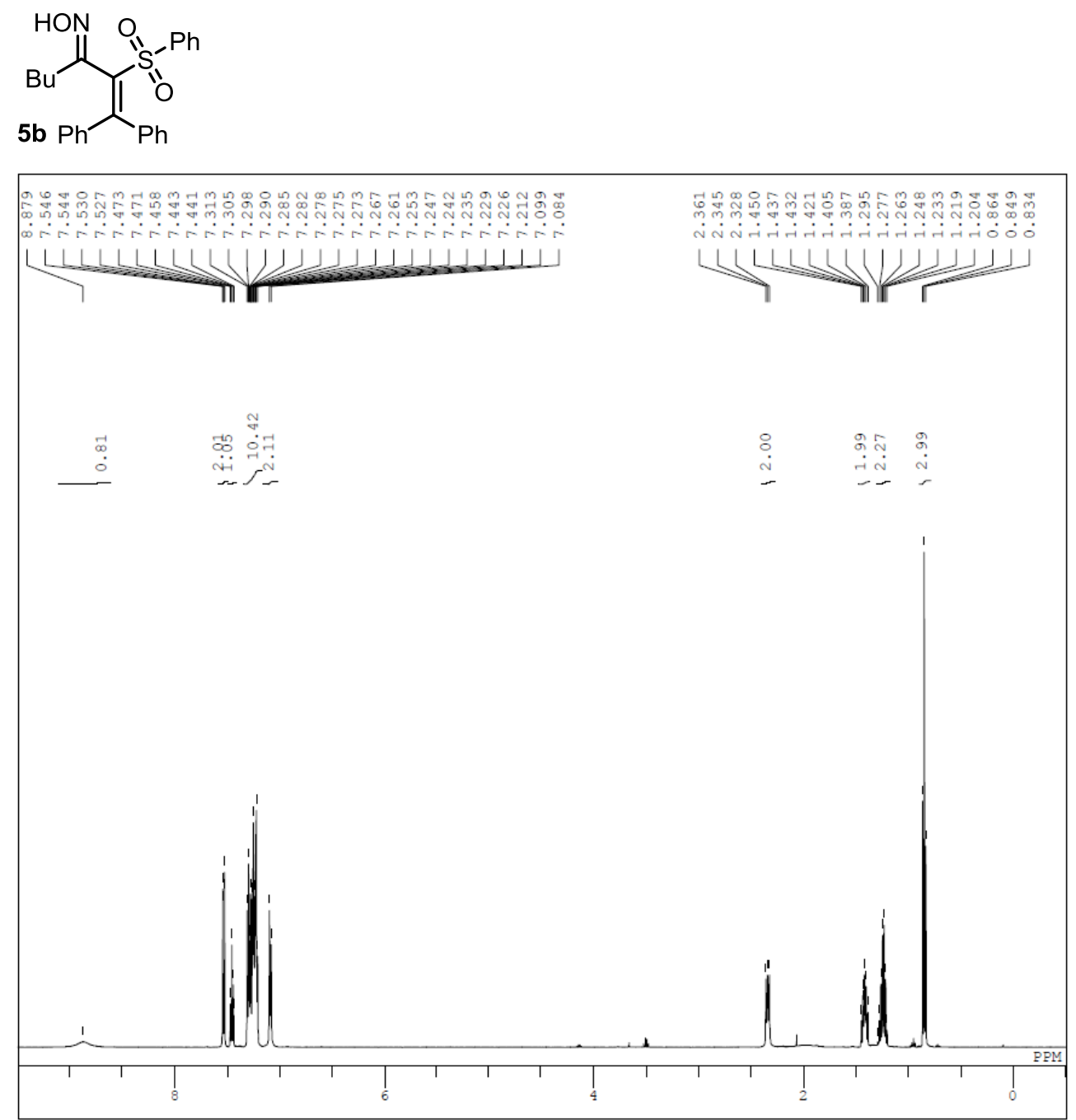

DFILE ky_01_075_s2-1-1.als

COMNI Ph_oxime

DATIM 2013-10-09 01:10:14

OBNUC $1 \mathrm{H}$

$\begin{array}{lc}\text { EXMOD } & \text { proton.jxp } \\ \text { OBFRQ } & 500.16 \mathrm{MHz}\end{array}$

$\begin{array}{lr}\text { OBFRQ } & 500.16 \mathrm{MHz} \\ \text { OBSET } & 2.41 \mathrm{KHz}\end{array}$

OBEIN $\quad 6.01 \mathrm{~Hz}$

$\begin{array}{lr}\text { POINI } & 13107\end{array}$

FREQU
SCANS

ACQTM $\quad 1.7459 \mathrm{sec}$

PD $5.0000 \mathrm{sec}$

PW1 1

IRNUC $1 \mathrm{H}$

SIVNI CDCL 3

EXREF $7.26 \mathrm{ppm}$

$\begin{array}{ll}\text { EXREF } & 7.26 \mathrm{ppm} \\ \mathrm{BF} & 0.10 \mathrm{~Hz}\end{array}$

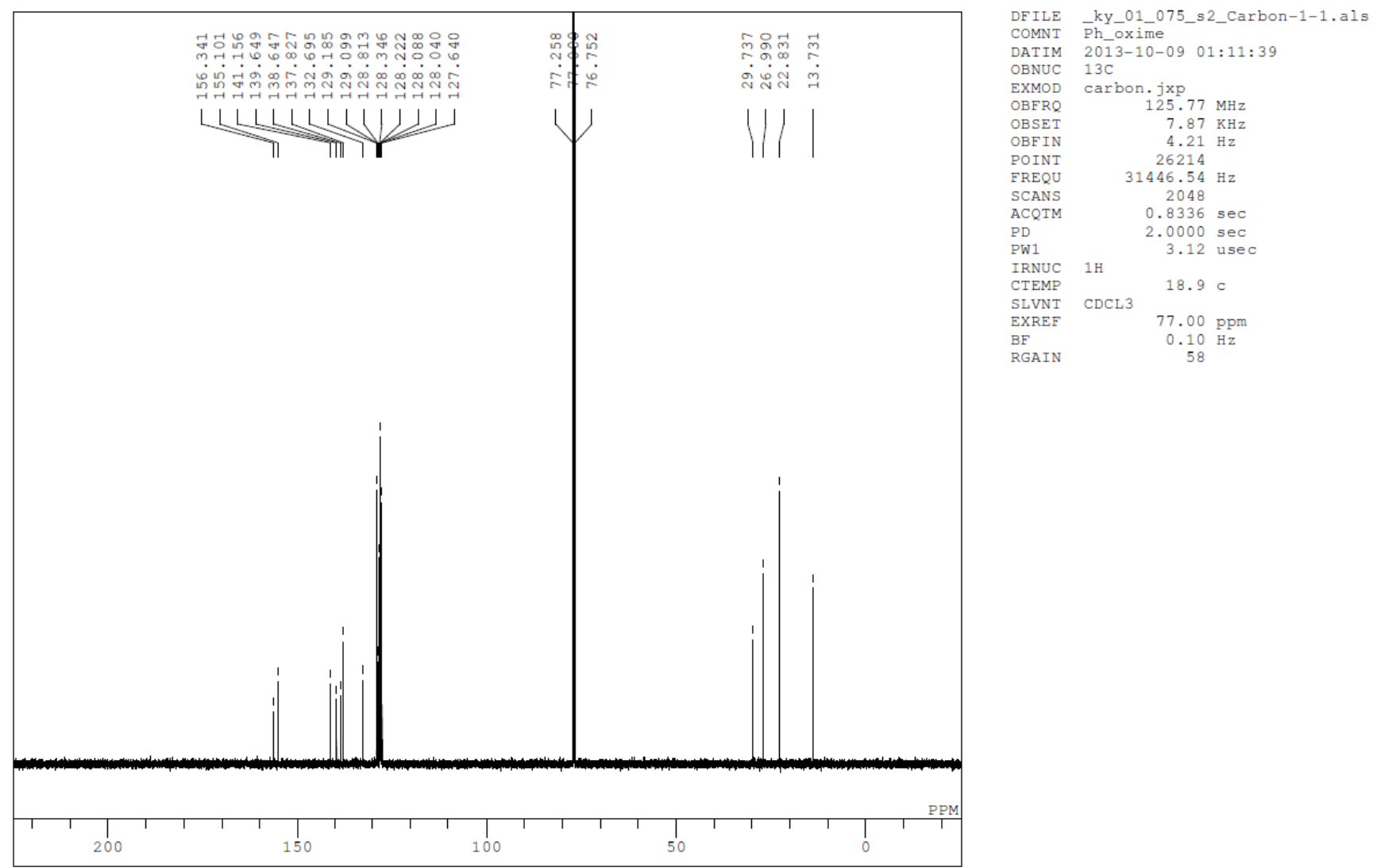


<smiles>N=C(Cc1ccccc1)C(=NO)C(=C(c1ccccc1)c1ccccc1)S(=O)(=O)c1ccc(Cl)cc1</smiles>
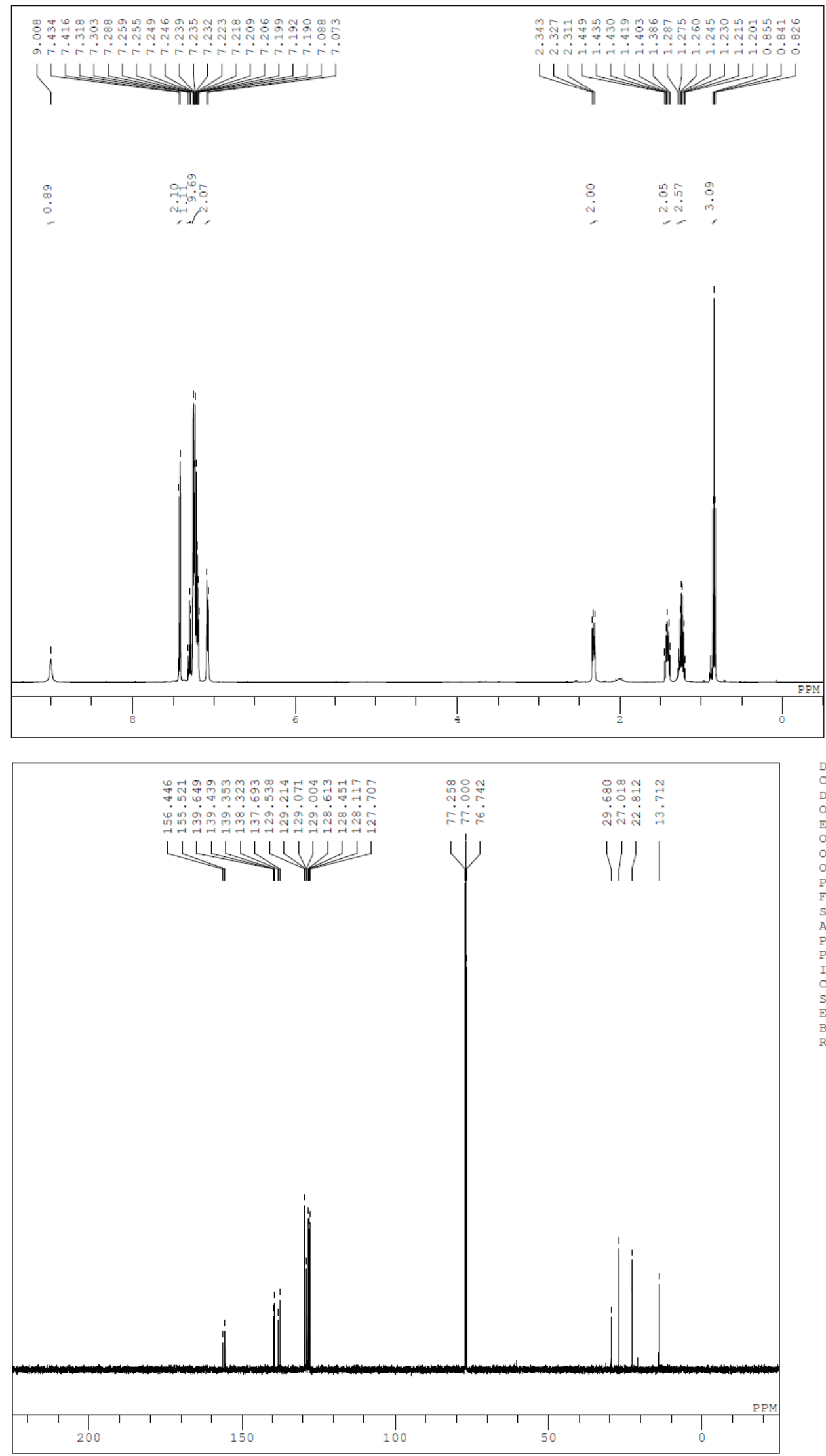

DFILE_ky_01_072_1_Carbon-1-1.als

COMNT P_ClPh_oxime

DATIM 2013-10-04 22:13:44

OBNUC $13 \mathrm{C}$

EXMOD Carbon. $\mathrm{jxp}$

OBFRQ $\quad 125.77 \mathrm{MHz}$

OBSET $7.87 \mathrm{KH}$

OBFIN $4.21 \mathrm{~Hz}$

POINT 26214

FREQU $31446.54 \mathrm{~Hz}$

SCANS 583

ACQTM $0.8336 \mathrm{sec}$

PD $2.0000 \mathrm{sec}$

PW1 3.12 usec

IRNUC $1 \mathrm{H}$

SLVNT CDCL3

EXREF CDCL3 77.00 PPA

$\begin{array}{ll}\mathrm{BF} & 0.10 \mathrm{~Hz}\end{array}$

$77.00 \mathrm{ppm}$ 

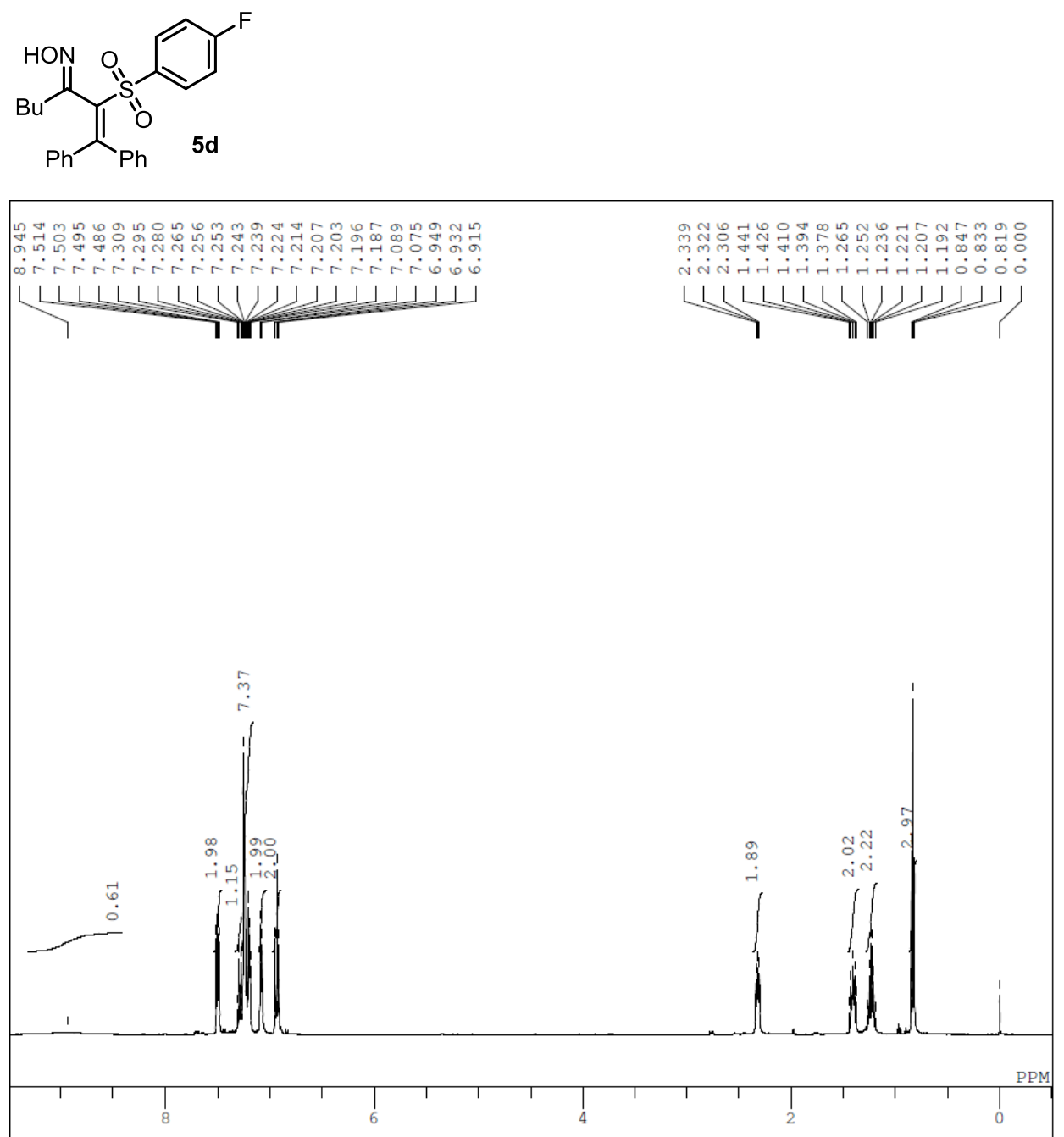

DFILE -ym_06_022_2-1-1.als

COMNT single_pulse

DATIM 2015-02-14 16:01:31

OBNUC 1

EXMOD proton.jxp

OBFRQ $\quad 500.16 \mathrm{MHz}$

$2.41 \mathrm{KHz}$

OBFIN $\quad 6.01 \mathrm{~Hz}$

$\begin{array}{lr}\text { POINT } & 13107\end{array}$

FREQU $\quad 7507.51 \mathrm{~Hz}$

$\begin{array}{lr}8 \\ \text { SCANS } & 8 \\ \text { ACOTM } & 1.7459 \mathrm{sec}\end{array}$

PW1

6.22 used

$\begin{array}{lll}\text { IRNUC } & 1 \mathrm{H} & 17.5 \mathrm{C}\end{array}$

SLVNT CDCL 3

EXREF

$\mathrm{BF}$

BGAIN

$0.00 \mathrm{ppm}$

$0.10 \mathrm{~Hz}$

(

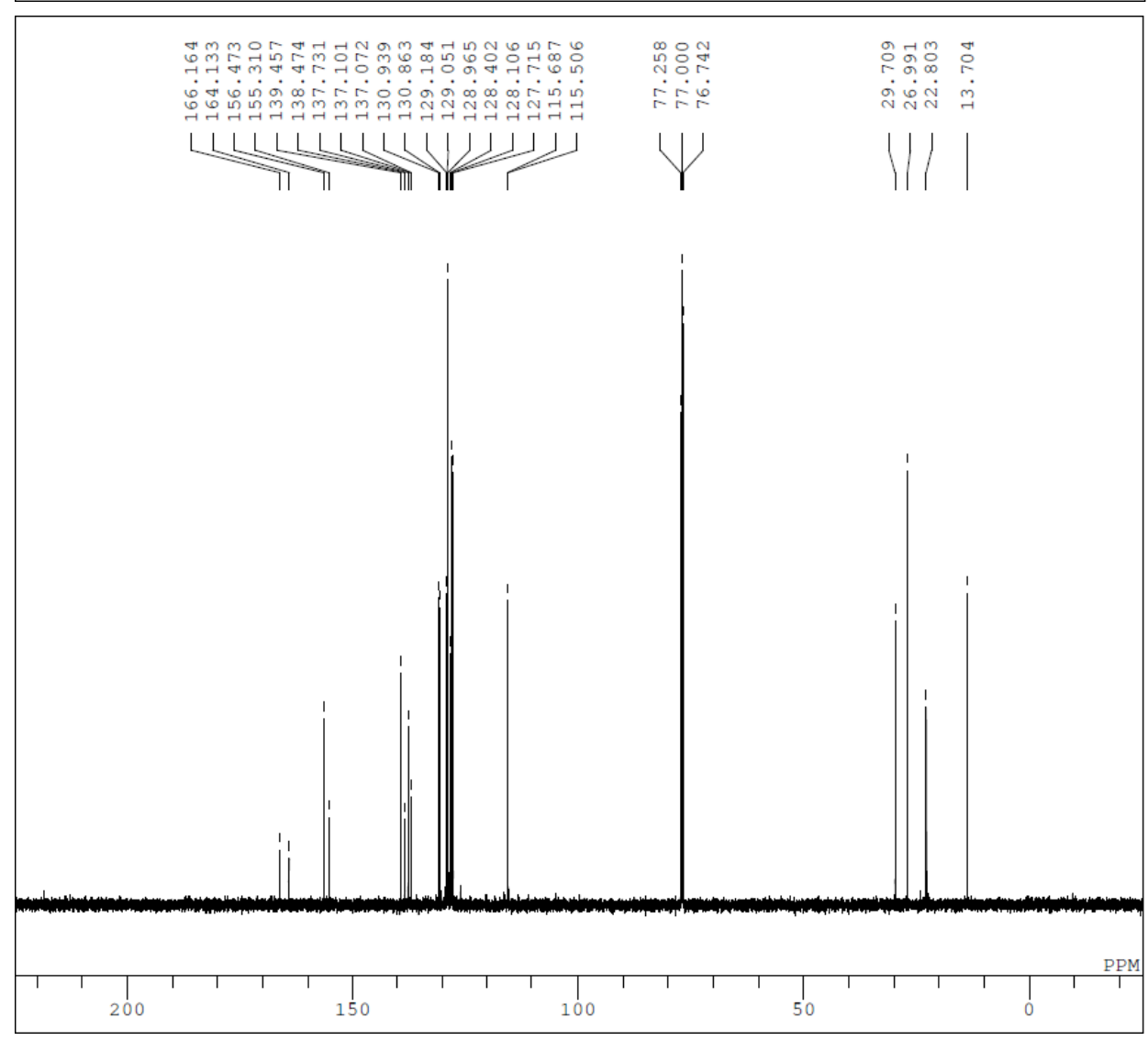

DFILE ym_06_022 2 2 13C-1-1.jdf

COMNT single pulse decoupled gated

DATIM 2015-02-14 16:02:55

OBNUC $13 \mathrm{C}$

EXMOD carbon jxp

OBERQ $\quad 125.77 \mathrm{MHz}$

OBSET $\quad 7.87 \mathrm{KHz}$

OBFIN $4.21 \mathrm{~Hz}$

POINT 32767

FREQU $\quad 39308.18 \mathrm{~Hz}$

SCANS 282

ACQTM $\quad 0.8336 \mathrm{sec}$

$\begin{array}{ll}\text { PD } & 2.0000 \mathrm{sec} \\ \text { PW1 } & 3.12 \text { usec }\end{array}$

IRNUC 1H

CTEMP

SLVNT

EXRE
BF

BF
RGAIN

$17.8 \mathrm{c}$

CDCL3

$77.00 \mathrm{ppm}$

$0.10 \mathrm{~Hz}$ 

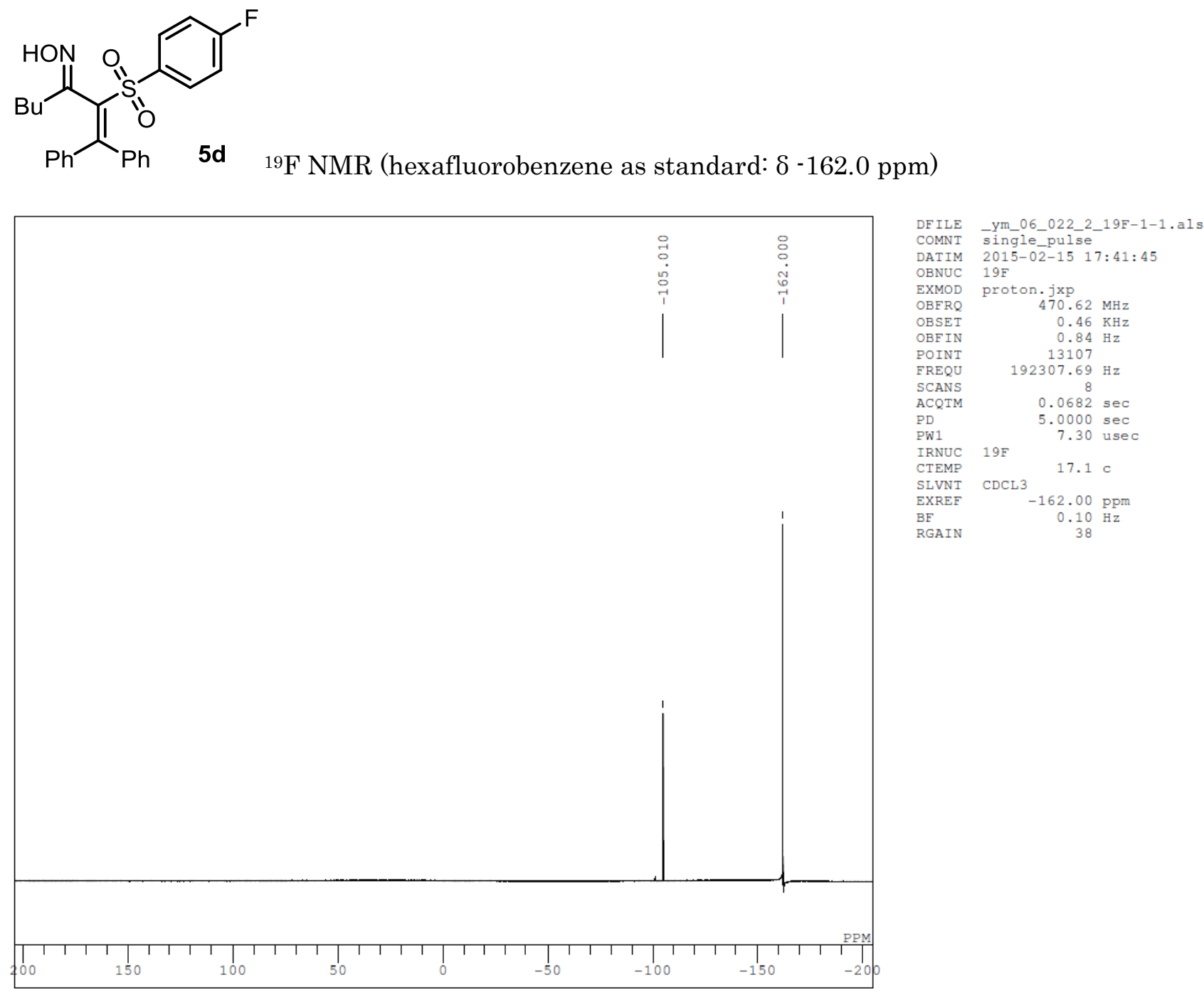
(OMe
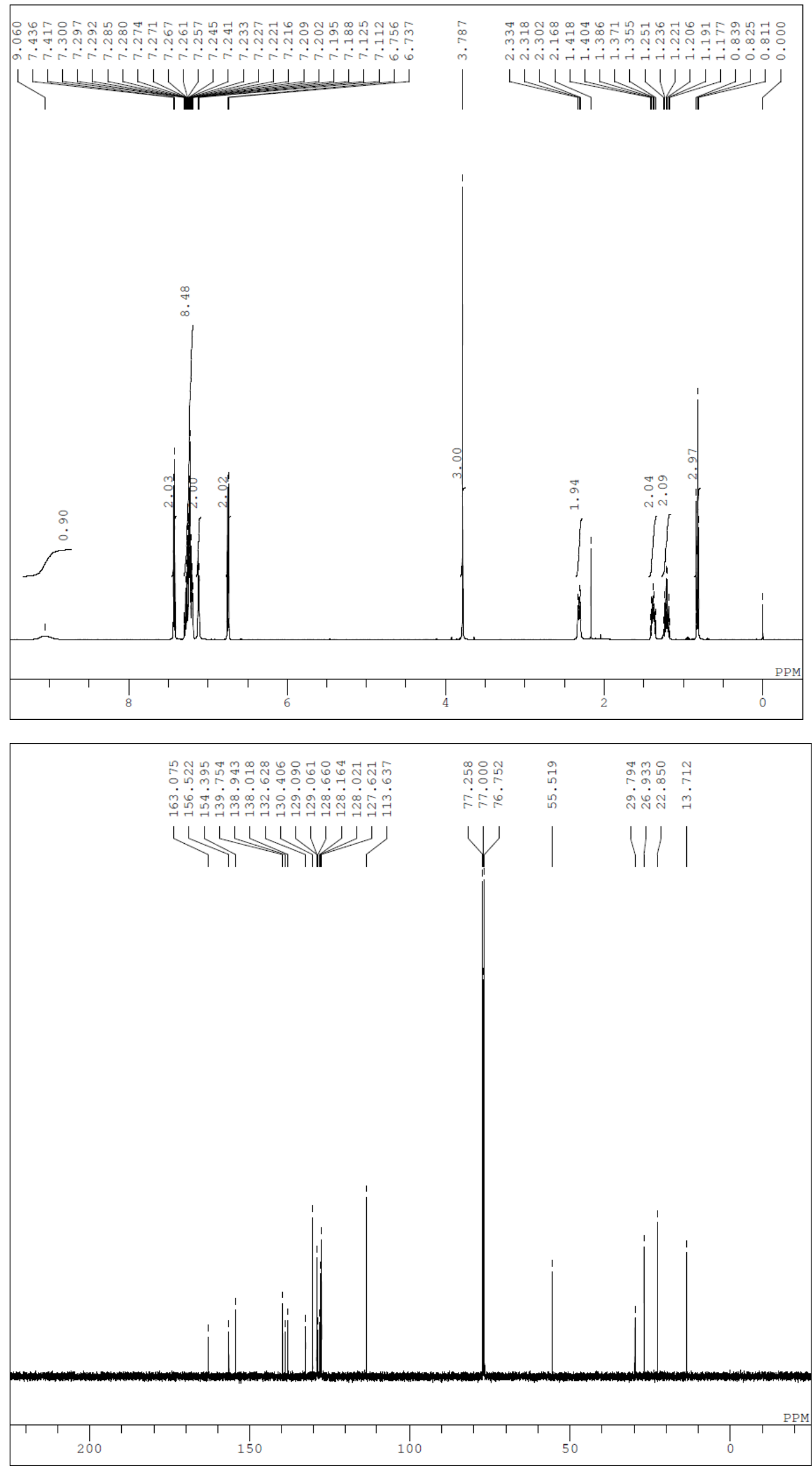

DFILE -Ym_06_019_1-1-1.als

COMNI single_pulse

DATIM 201

OBNUC 1

EXMOD proton.jxp

$\begin{array}{ll}\text { OBFRQ } & 500.16 \mathrm{MHz} \\ \text { OBSET } & 2.41 \mathrm{KHz}\end{array}$

OBFIN $6.01 \mathrm{~Hz}$

POINT 13107

FREQU $\quad 7507.51 \mathrm{~Hz}$

$\begin{array}{ll}\text { SCANS } & 8 \\ \text { ACQTM } & 1.7459 \mathrm{sec}\end{array}$

ACQTM $5.7459 \mathrm{sec}$

$\begin{array}{lr}\text { PD } & 5.0000 \text { sec } \\ \text { PW1 } & 6.22 \text { usec }\end{array}$

IRNUC $1 \mathrm{H}$

CTEMP $18.9 \mathrm{C}$

SLVNT CDCL 3

EXREF

BF

$0.00 \mathrm{ppm}$

$0.10 \mathrm{~Hz}$
DFILE ym_06_016_1_13C-1-1.als

COMNT single pulse decoupled gated

DATIM 2014-09-03 09:15:34

OBNUC 13C

EXMOD carbon.jxp

OBFRQ $\quad 125.77 \mathrm{MHz}$

OBSET $\quad 7.87 \mathrm{KHz}$

OBFIN $4.21 \mathrm{~Hz}$

POINT 26214

FREQU $\quad 31446.54 \mathrm{~Hz}$

SCANS 512

ACQTM $\quad 0.8336 \mathrm{sec}$

PW1

IRNUC 1H

CTEMP

SLVNT

EXREF

$\mathrm{BF}$

RGAIN

CDCL 3

3.12 use

$20.0 \mathrm{c}$

$77.00 \mathrm{ppm}$

$0.10 \mathrm{~Hz}$ 


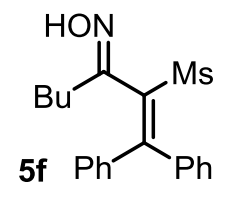
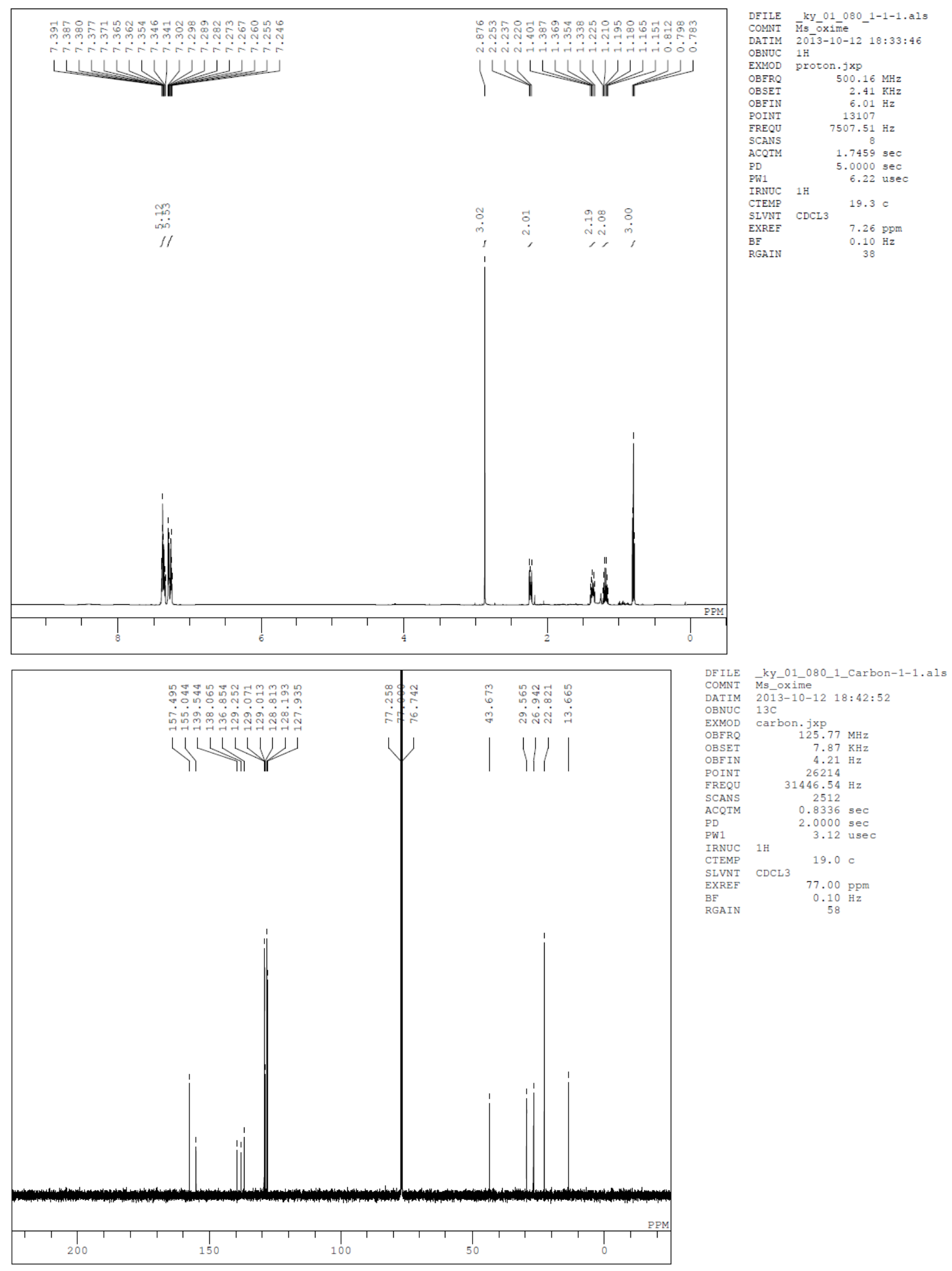

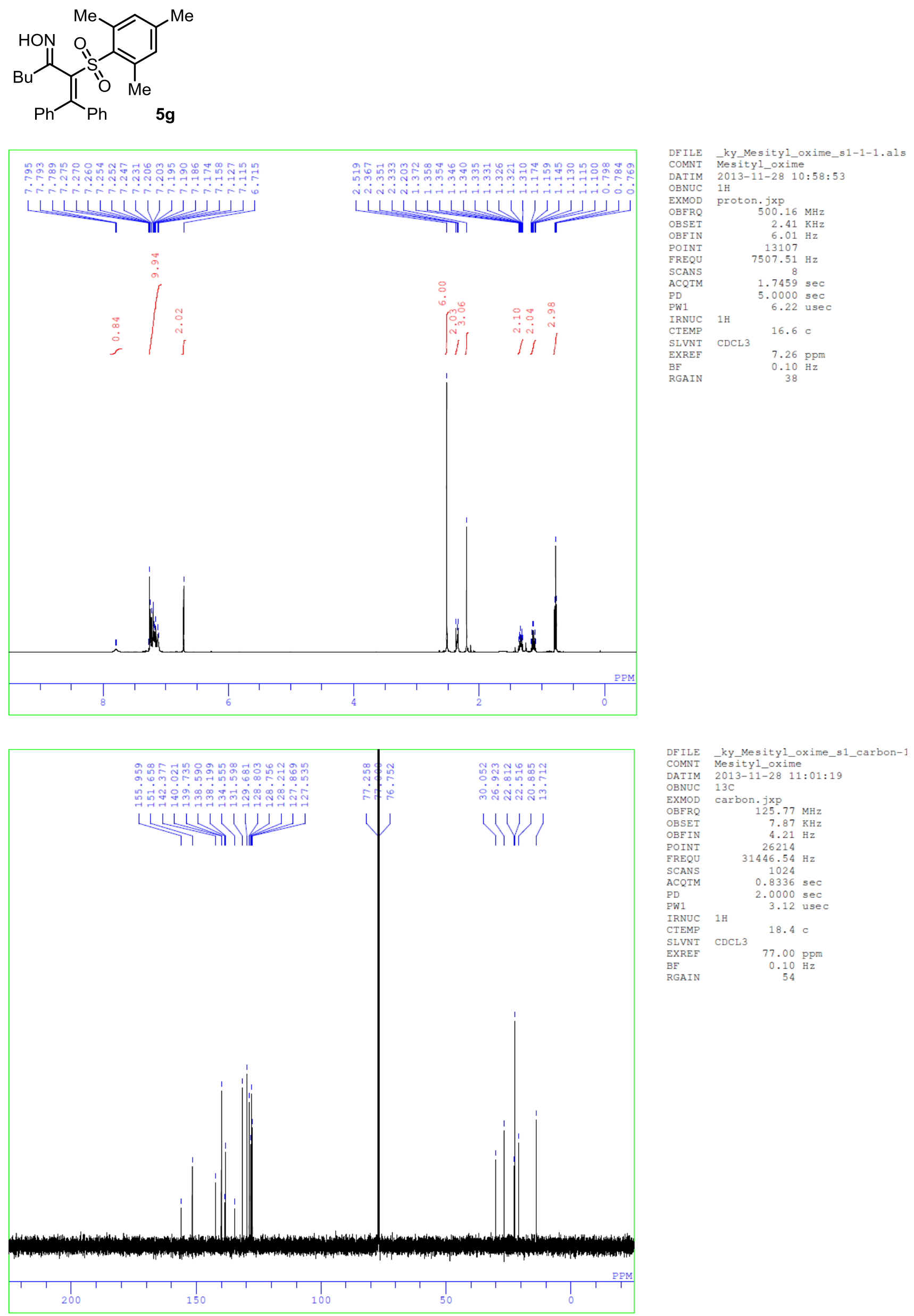

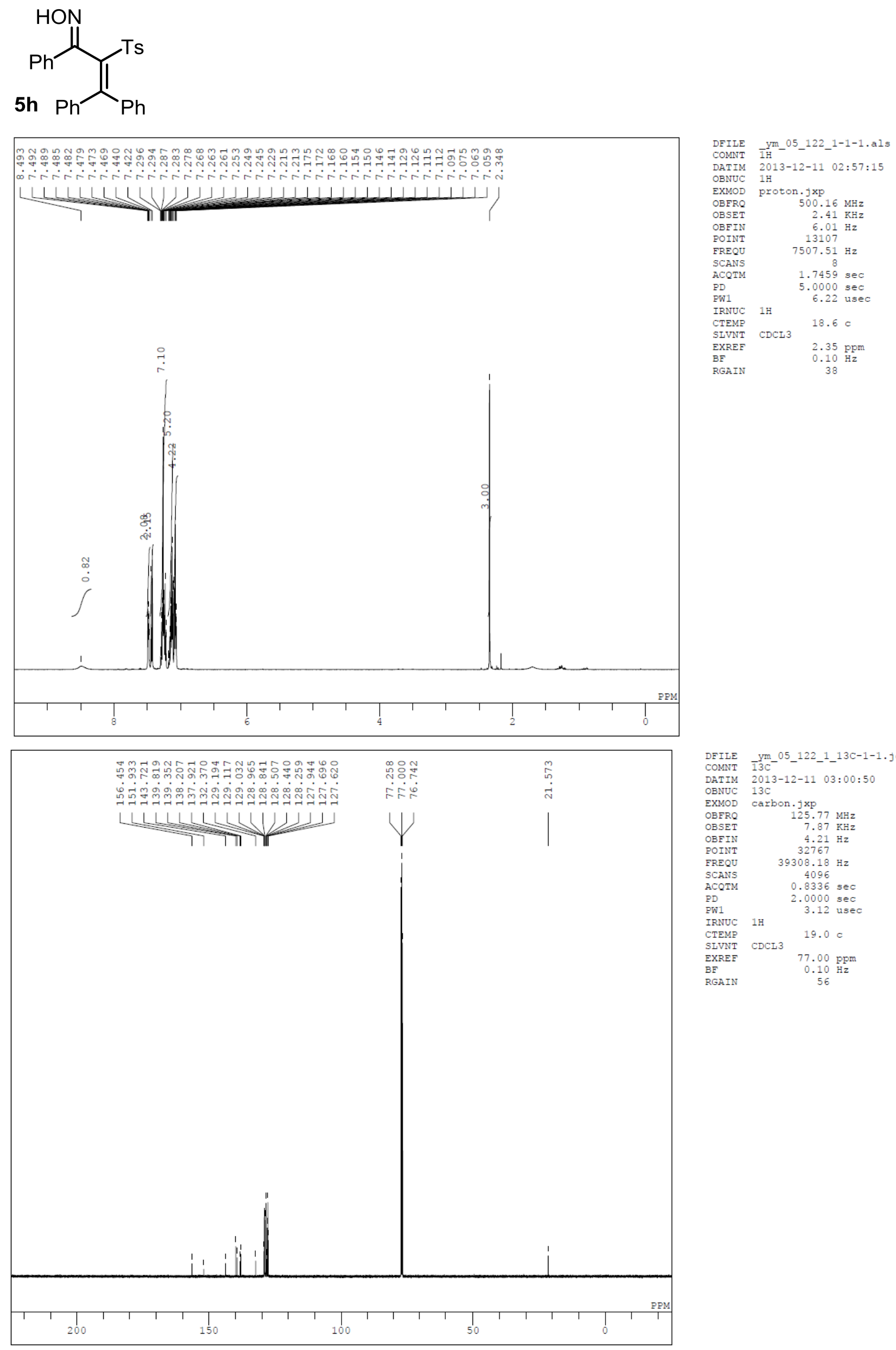

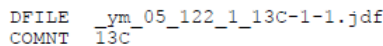
DATIM 2013-12-11 03:00:50
OBNUC $13 \mathrm{C}$
EXMOD carbon.jXp
$\begin{array}{lr}\text { OBFRQ } & 125.77 \mathrm{MHz} \\ \text { OBSET } & 7.87 \mathrm{KHz}\end{array}$
OBFIN $\quad 4.21 \mathrm{~Hz}$ POINT 32767
FREQU $39308.18 \mathrm{~Hz}$
SCANS 4096
ACQTM $\quad 0.8336 \mathrm{sec}$
PD 2.0000 sec
PW1 3.12 usec
IRNUC $1 \mathrm{H}$
19.0 C
SIVNT CDCL 3
EXRE
RGA
$77.00 \mathrm{ppm}$
$.00 \mathrm{ppm}$
$0.10 \mathrm{~Hz}$
56



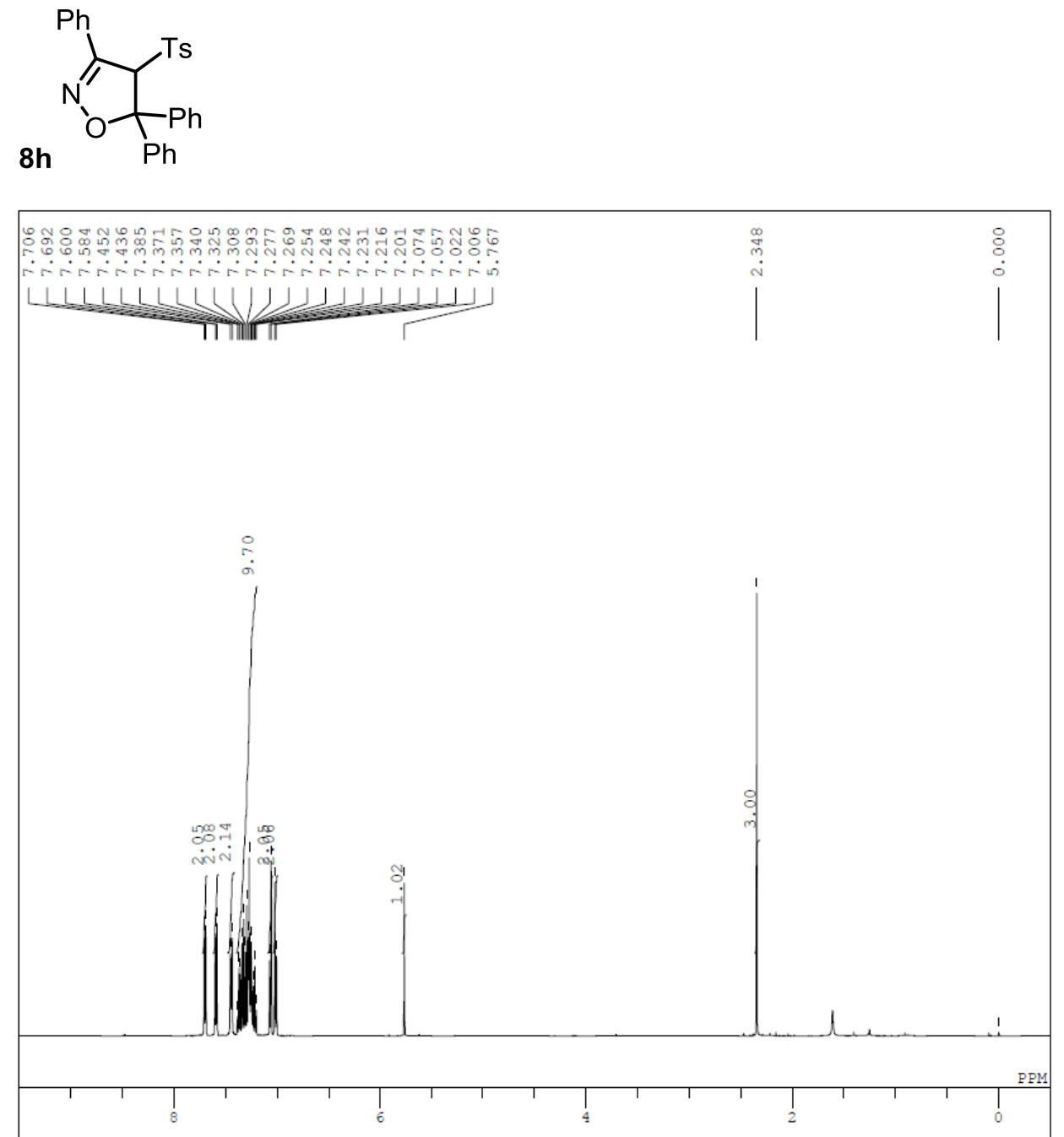

DFILE YM_05_058_3-1-1.als

DATIM 2013-09-10 09:05:43

OBNUC $1 \mathrm{H}$

EXMOD proton.jxp

$\begin{array}{lr}\text { OBFRQ } & 500.16 \mathrm{MHz} \\ \text { OBSET } & 2.41 \mathrm{KHz}\end{array}$

OBFIN $6.01 \mathrm{~Hz}$

POINT

FREQU $\quad 7507.51 \mathrm{~Hz}$

$\begin{array}{lr}8 \\ \text { SCANS } & 8 \\ \text { ACOTM } & 1.7459 \mathrm{sec}\end{array}$

$\begin{array}{ll}\text { ACQTM } & 1.7459 \mathrm{sec} \\ \text { PD } & 5.0000 \mathrm{sec}\end{array}$

PW1 6.22 usec

IRNUC $1 \mathrm{H}$

SLVNT CDCL 3

$0.00 \mathrm{ppm}$

$\begin{array}{lr}\text { BF } & 0.10 \mathrm{~Hz} \\ \text { RGAIN } & 34\end{array}$

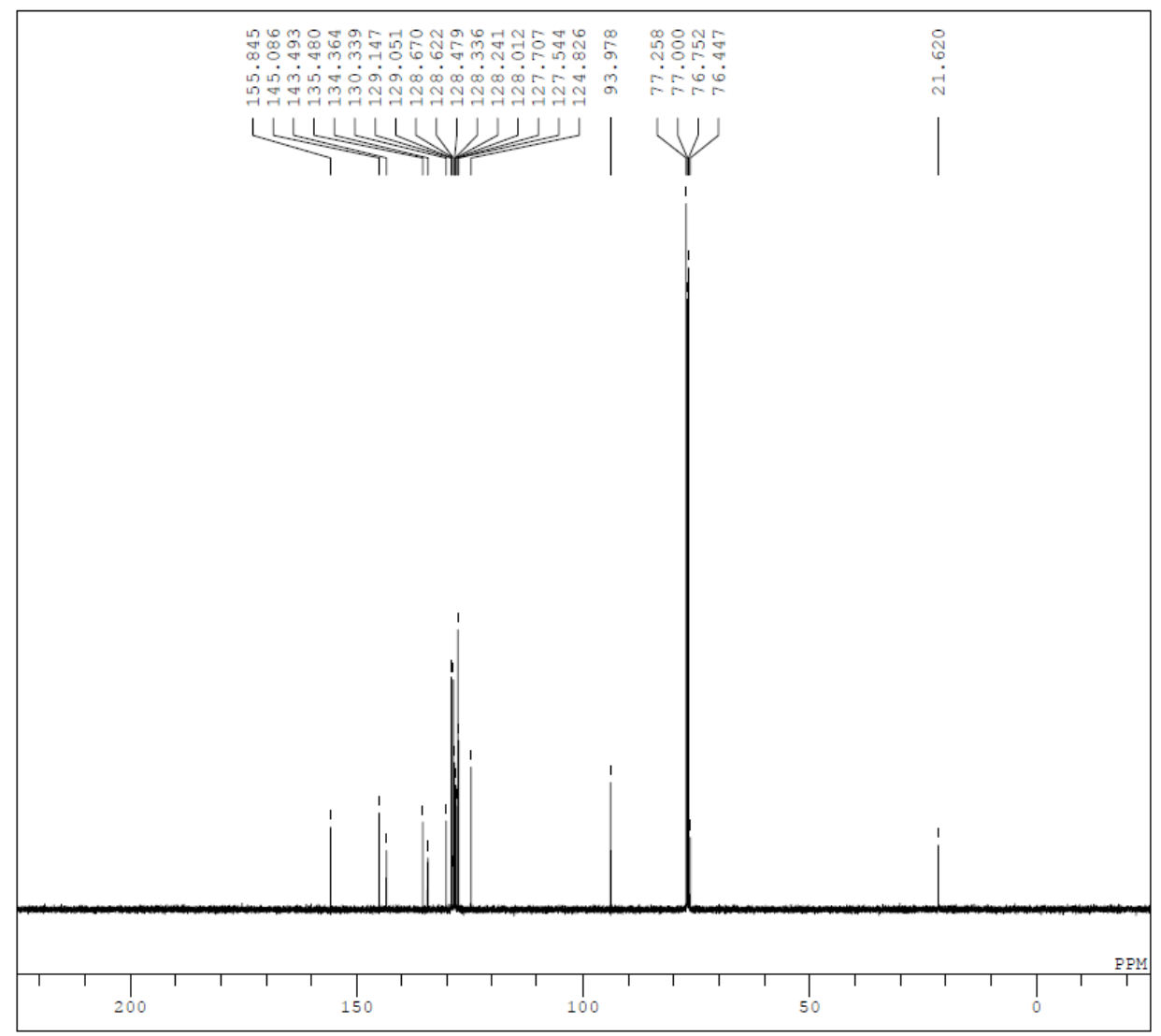

DFILE_ym_05_058_3_13C-1-1.als

DATIM 2013-09-11 13:19:31

OBNUC $13 \mathrm{C}$

EXMOD carbon.jxp

OBFRQ $125.77 \mathrm{MHz}$

$\begin{array}{lr}\text { OBFRQ } & 125.77 \mathrm{MHz} \\ \text { OBSET } & 7.87 \mathrm{KHz}\end{array}$

OBFIN $\quad 4.21 \mathrm{~Hz}$

POINT 26214

FREQU 3146.54

$\begin{array}{lr}\text { ACQTM } & 0.8336 \mathrm{sec} \\ \text { PD } & 2.0000 \mathrm{sec} \\ \text { PW1 } & 3.12\end{array}$

PW1 3.12 usec

IRNUC 1H $19.3 \mathrm{C}$

SLVNT CDCL3

EXREF $\quad 77.00 \mathrm{ppm}$

$B F$

$77.00 \mathrm{ppm}$
$0.10 \mathrm{~Hz}$
58

RGAIN 
<smiles>[3H]C(C(O)=C([As])c1ccccc1)=C(c1ccccc1)c1ccc(Br)cc1</smiles>

$5 i$

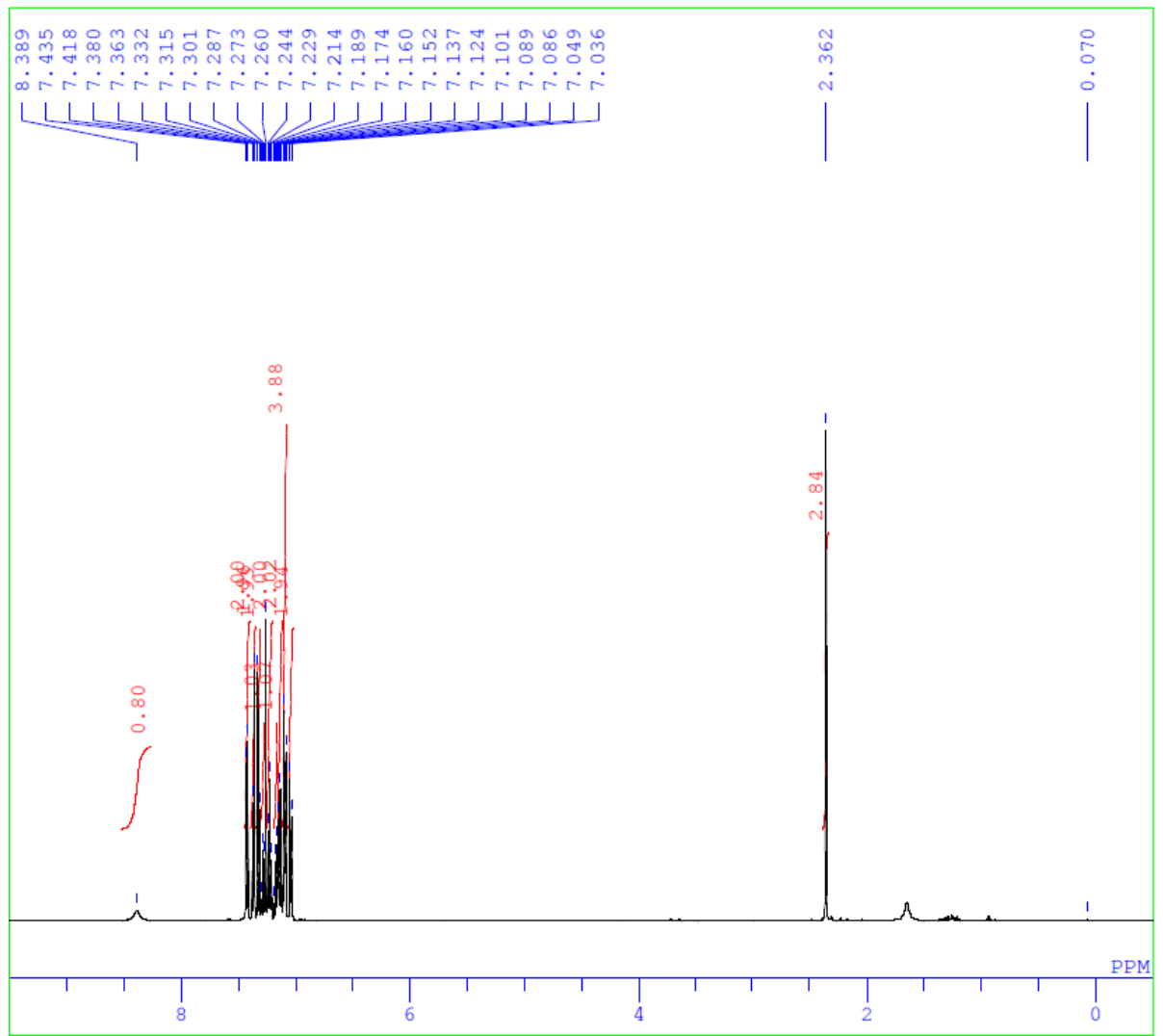

DFILE $-\mathrm{ym}$ C5_130_5-1-1.als
COMNT $1 \mathrm{H}$

DATIM 2013-12-26 21:21:34

OBNUC $1 \mathrm{H}$

EXMOD proton.jxp

OBFRQ $500.16 \mathrm{MHz}$

$\begin{array}{rr}\text { OBERQ } & 500.16 \mathrm{MHz} \\ \text { OBSET } & 2.41 \mathrm{KHz}\end{array}$

OBFIN $\quad 6.01 \mathrm{~Hz}$

POINT $\quad 13107$

FREQU $\quad 7507.51 \mathrm{~Hz}$

SCANS

ACQTM

PD

$1.7459 \mathrm{sec}$

$1.7459 \mathrm{sec}$
$5.0000 \mathrm{sec}$

PW1 6.22 usec

IRNUC $1 \mathrm{H}$

SLVNT CDCL3

EXREF

EXR
BF

BF

$19.6 \mathrm{C}$

$7.26 \mathrm{ppm}$

$0.10 \mathrm{~Hz}$

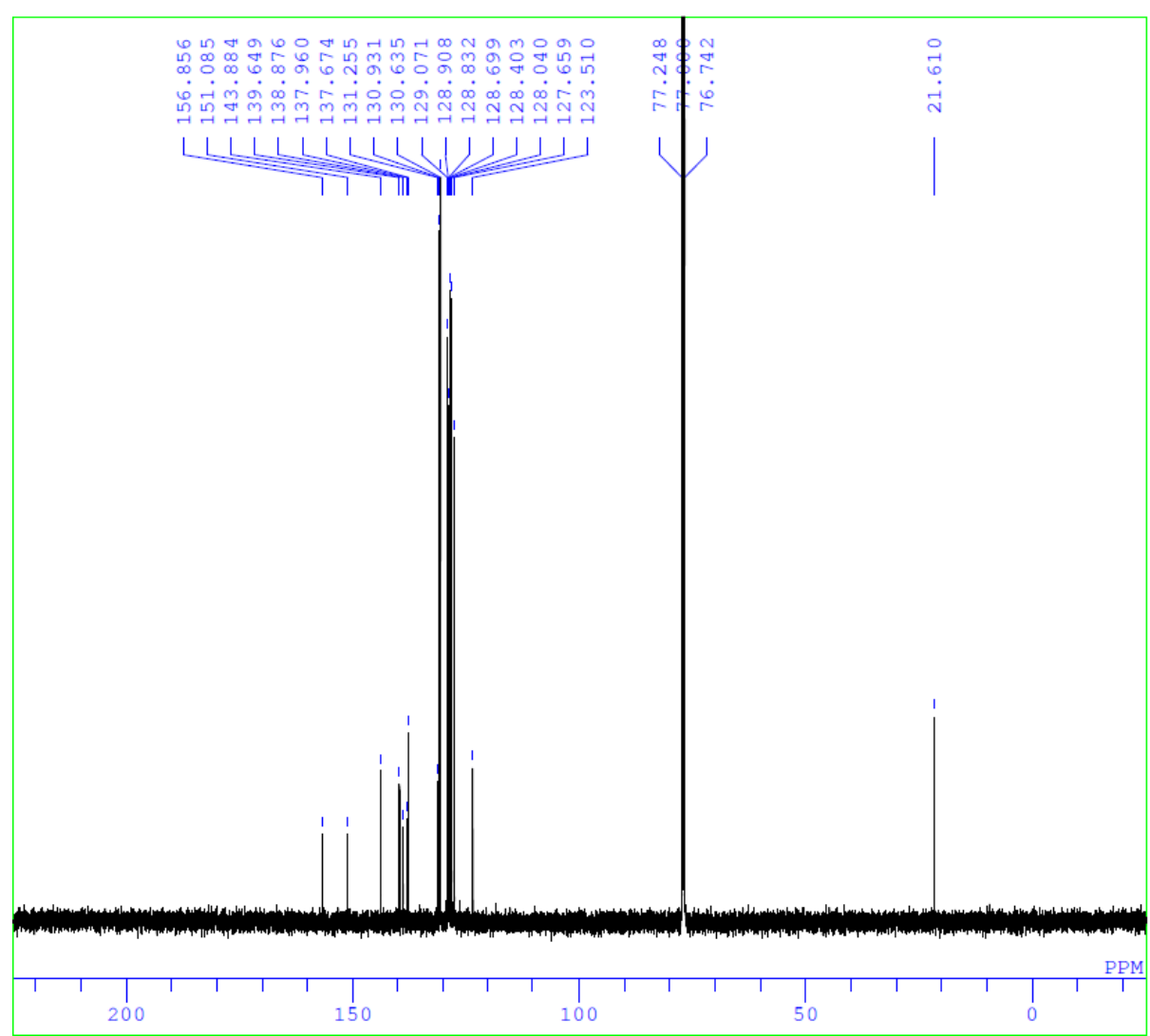

DFILE -ym_05_130_5_13C-1-1.als

COMNT $13 \mathrm{C}$

DATIM 2013-12-26 23:04:13

OBNUC 13C

EXMOD carbon.jxp

OBFRO $\quad 125.77 \mathrm{MHz}$

$\begin{array}{lr}\text { OBFRQ } & 125.77 \mathrm{MHz} \\ \text { OBSET } & 7.87 \mathrm{KHz}\end{array}$

$\begin{array}{ll}\text { OBSET } & 7.87 \mathrm{KHz} \\ \text { OBFIN } & 4.21 \mathrm{~Hz}\end{array}$

$\begin{array}{lr}\text { OBEIN } & 4.21 \\ \text { POINT } & 26214\end{array}$

$\begin{array}{lr}\text { POINT } & 26214 \\ \text { FREQU } & 31446.54 \mathrm{~Hz}\end{array}$

SCANS 8192

ACQTM $0.8336 \mathrm{sec}$

PD $2.0000 \mathrm{sec}$

PW1 $\quad 3.12$ usec

IRNUC $1 \mathrm{H}$

CTEMP

SLVNT CDCL3

EXREF

$\mathrm{BF}$

$15.3 \mathrm{c}$

$77.00 \mathrm{ppm}$

RGAIN $\quad \begin{aligned} & 0.10 \mathrm{~Hz} \\ & 58\end{aligned}$ 

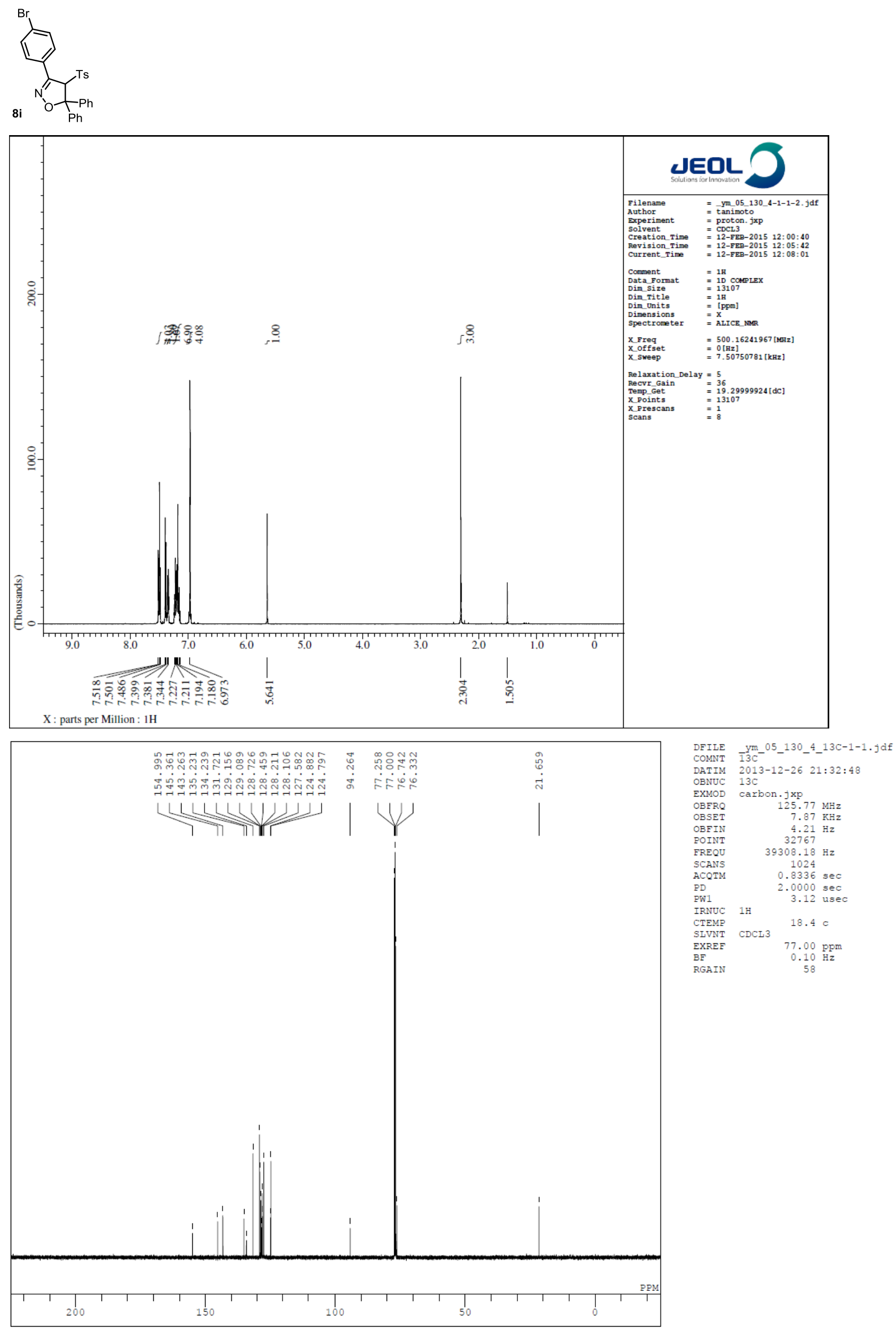
<smiles>[15NH]C(=C(C=O)c1ccccc1)c1ccccc1</smiles>

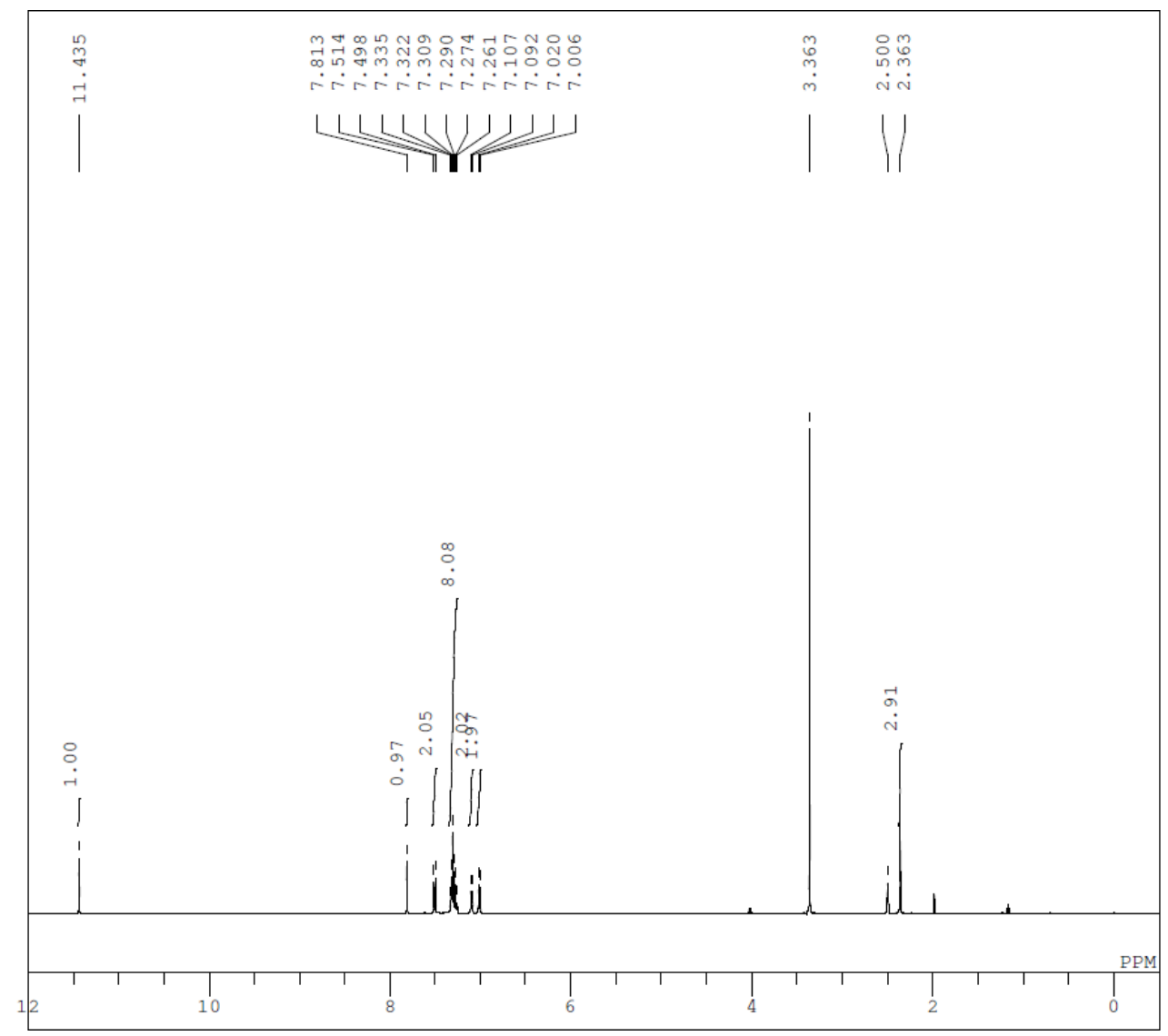

DFILE _ym_06_006_4-1-1.als

COMNT single_pulse

DATIM 2014-09-05 13:50:10

OBNUC $1 \mathrm{H}$

EXMOD proton.jxp

OBFRQ $\quad 500.16 \mathrm{MHz}$

OBSET $2.41 \mathrm{KHz}$

OBFIN

POIN

FREQU

SCANS

ACQTM

PD

IRNUC $1 \mathrm{H}$

CTEMP

SLVNT DMSO

EXREF

RGATN

$6.01 \mathrm{~Hz}$

13107

$7507.51 \mathrm{~Hz}$

1. $7459 \mathrm{sec}$

$5.0000 \mathrm{sec}$

$1 \mathrm{H}$

6.22 usec

$19.2 \mathrm{c}$

2. $50 \mathrm{ppm}$

$0.10 \mathrm{~Hz}$

36
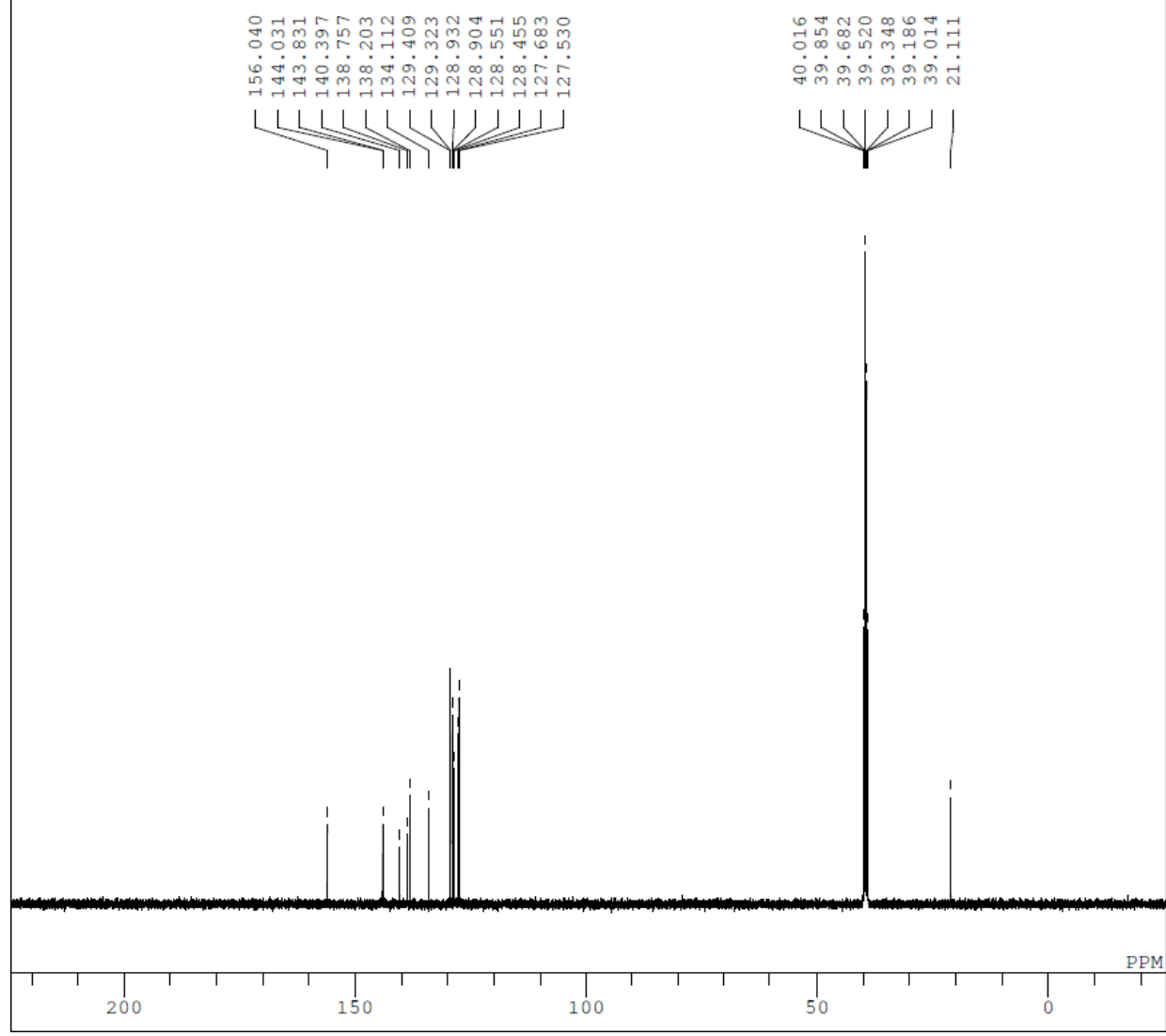

DFILE ym_06 006 3 13C-7-1.jdf

COMNT single pulse decoupled gated

DATIM 2015-02-11 13:40:41

OBNUC $13 \mathrm{C}$

EXMOD carbon, jXp

OBFRQ $\quad 125.77 \mathrm{MHz}$

$\begin{array}{lr}125.77 \mathrm{MHz} \\ \text { OBSET } & 7.87 \mathrm{KHz}\end{array}$

$\begin{array}{lr}\text { OBFIN } & 4.21 \mathrm{~Hz}\end{array}$

FREQU

FREQU

SCANS

$\mathrm{PD}$ $39308.18 \mathrm{~Hz}$

0.8336 sec

$0.8336 \mathrm{sec}$

IRNUC $1 \mathrm{H}$

SLVNT DMSO

EXREF

$\mathrm{BF}$

$18.0 \mathrm{C}$

$39.52 \mathrm{ppm}$ 
<smiles>OC(C([Al-])=C(c1ccccc1)c1ccccc1)C1CCCCC1</smiles>

5k

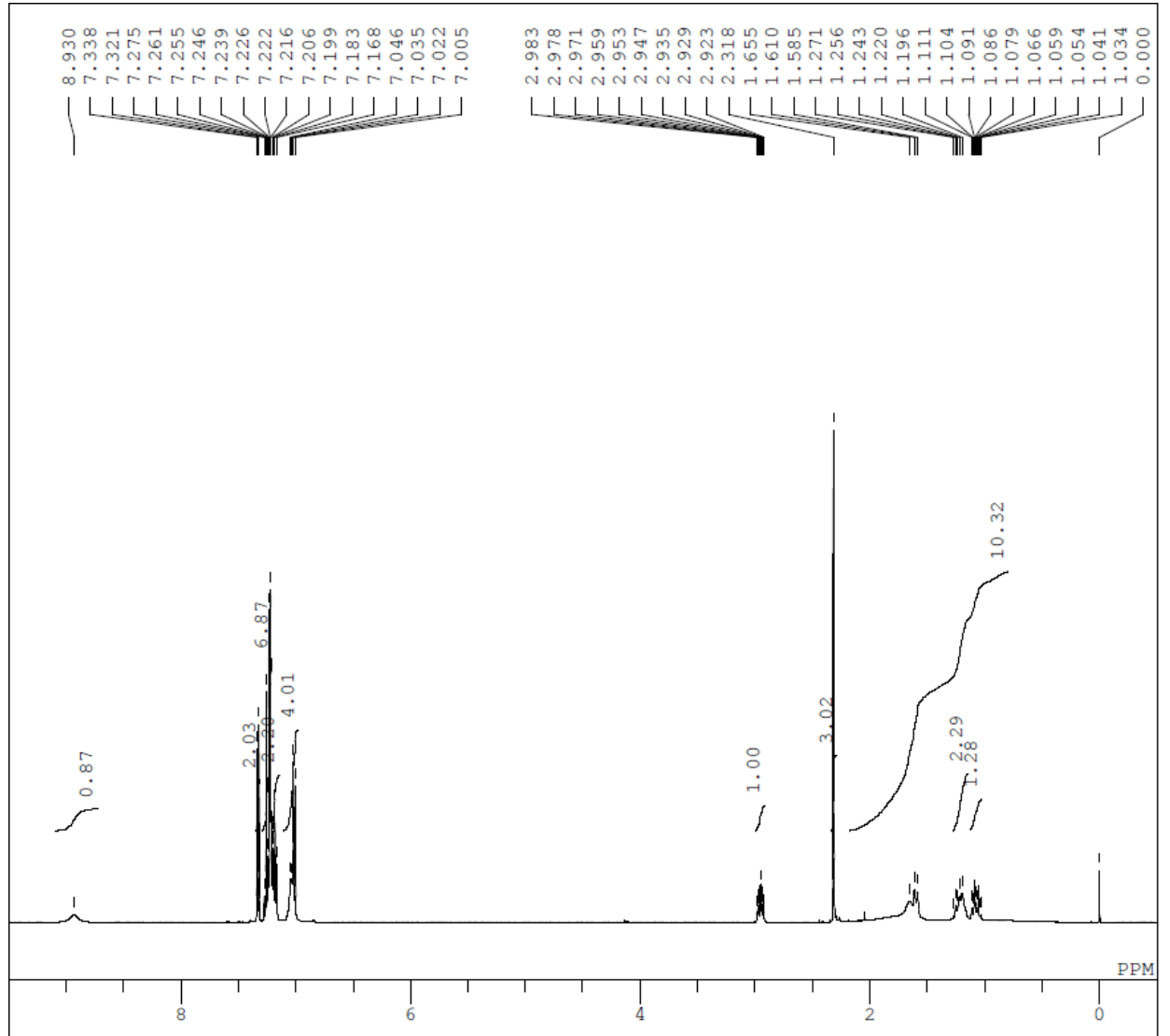

DEILE

-ym_06_005_2-1-1.als

COMNT single_pulse

DATIM 2014-08-21 13:30:56

DBNUC $1 \mathrm{H}$

EXMOD proton.jxp

OBFRQ $\quad 500.16 \mathrm{MHz}$

OBSET $2.41 \mathrm{KHz}$

OBFIN $\quad 6.01 \mathrm{~Hz}$

POINT

FREQU

SCANS

ACQTM

PD

PD 1

PW1
IRNUC 1 1H

CTEMP

SLVNT CDCL 3

13107

$7507.51 \mathrm{~Hz}$

8
$1.7459 \mathrm{sec}$

$5.0000 \mathrm{sec}$

6.22 usec

EXREF

BGAIN

$20.8 \mathrm{c}$

$0.00 \mathrm{ppm}$

$0.10 \mathrm{~Hz}$

\section{RGIN}

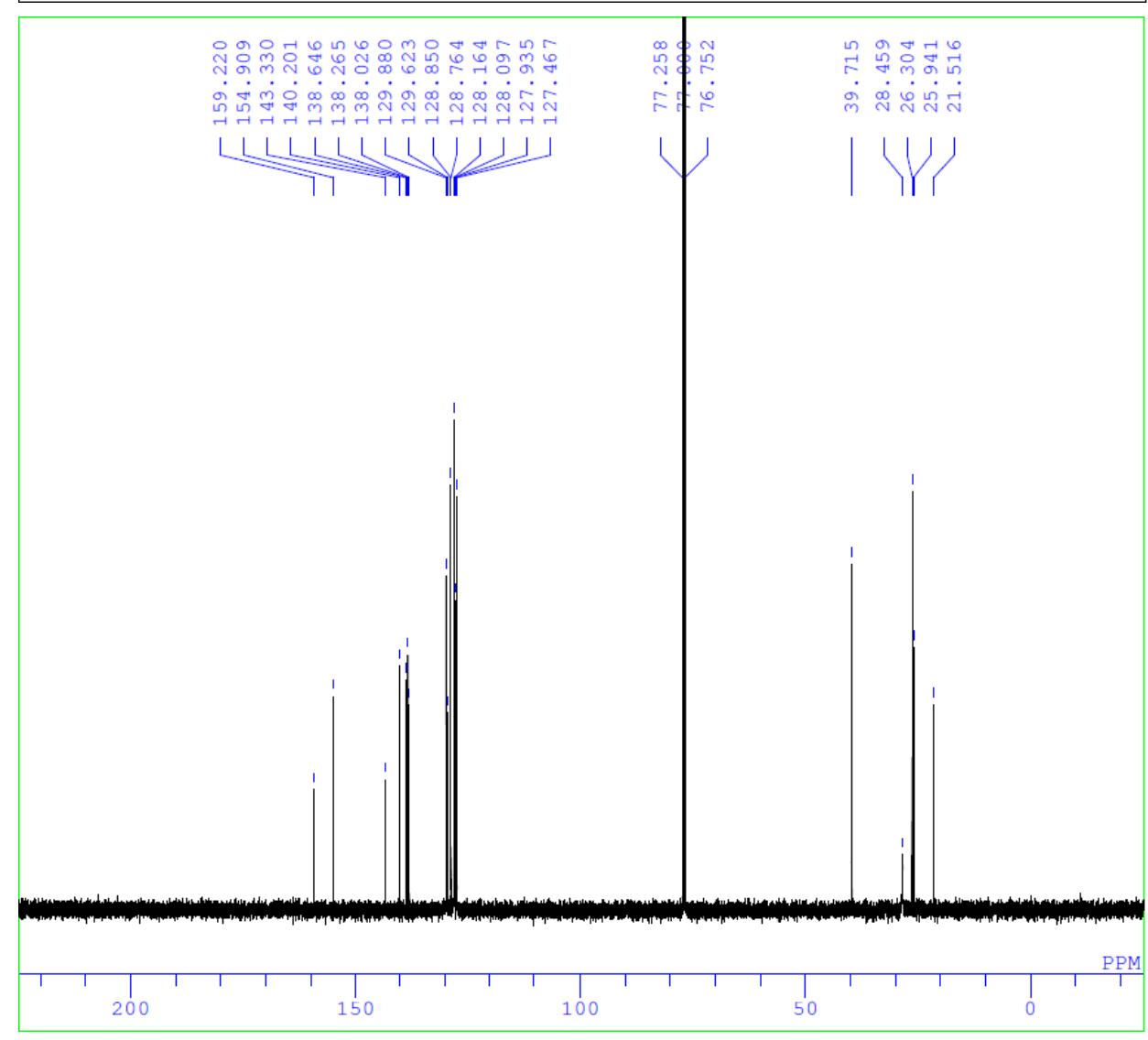

DFILE

COMNT

Tingle pulse decoupled

DATIM

DATIM

EXMOD

OBFRQ

OBSET

OBF IN

POINT

FREQU

SCANS

ACQTM

$\mathrm{PD}$

PW1

IRNUC

CTEMP

SLVNT

EXREF

BF

RGAIN

CDCL 3

$125.77 \mathrm{MHz}$

$7.87 \mathrm{KHz}$

$4.21 \mathrm{~Hz}$

32767

$39308.18 \mathrm{~Hz}$

1024

$0.8336 \mathrm{sec}$

$2.0000 \mathrm{sec}$

3.12 usec

$21.0 \mathrm{c}$

$77.00 \mathrm{ppm}$

$0.10 \mathrm{~Hz}$ 
<smiles>[15NH]/C(=C(\O)COc1ccccc1)c1ccccc1</smiles>

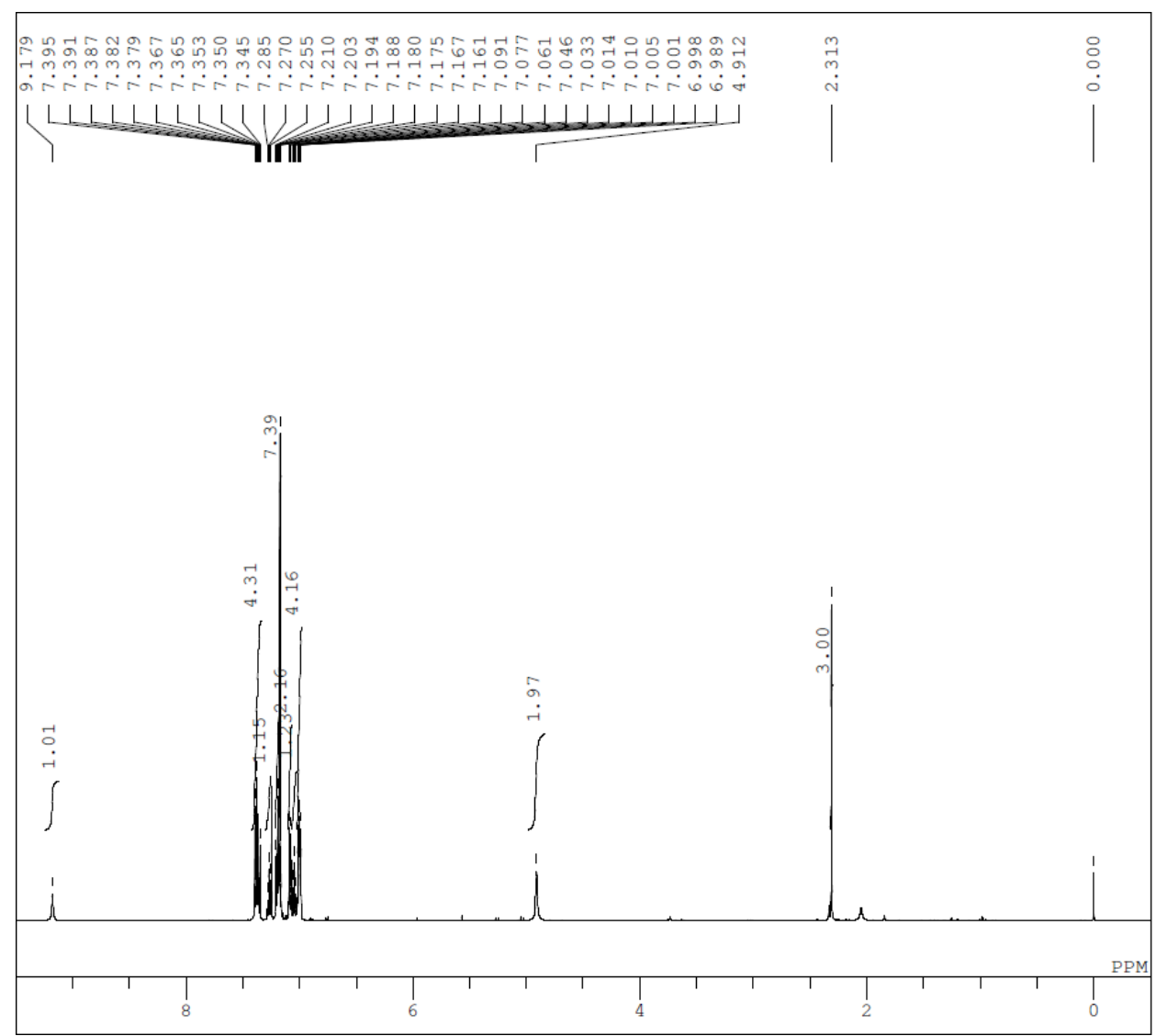

DFILE -YM_06_007_2-2-1.als

COMNT single_pulse

EXMOD proton, jxp

OBFRQ $\quad 500.16 \mathrm{MHz}$

OBSET $2.41 \mathrm{KHz}$

POINT 13107

FREQU $\quad 7507.51 \mathrm{~Hz}$

SCANS 8

ACQTM $\quad 1.7459 \mathrm{sec}$

$\begin{array}{lr}\text { PD } & 5.0000 \text { sec } \\ \text { PW } & 6.22 \text { usec }\end{array}$

IRNUC $1 \mathrm{H}$

19.30

SLVNT CDCL

$\mathrm{BF}$

$0.00 \mathrm{ppm}$

DFILE ym_06_007_2_13C-2-1.als

COMNT single pulse decoupled gated

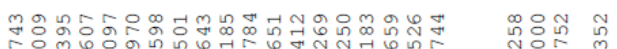

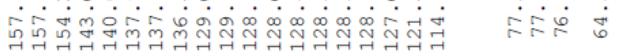

DATTM 2014-08-26 $11: 06: 07$

OBNUC 130

EXMOD carbon. jxp

OBFRQ $125.77 \mathrm{MHz}$

125.77
$\mathrm{MHZ}$

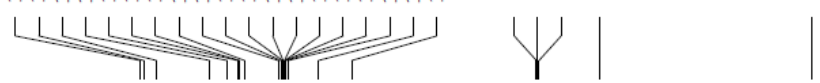

POINT

PREQU

SCANS

$\mathrm{ACQTM}$

PD

PW1

CTEMP

SLVNT

EXREF

$\mathrm{BF}$

$7.87 \mathrm{KHz}$
$4.21 \mathrm{~Hz}$

4.21

$31446.54 \mathrm{~Hz}$

1024
0.8336

$0.8336 \mathrm{sec}$

$2.0000 \mathrm{sec}$

$1 \mathrm{H}$

3.12 usec

CDCL3

$19.7 \mathrm{C}$

$77.00 \mathrm{ppm}$

$0.10 \mathrm{~Hz}$

RGAIN 


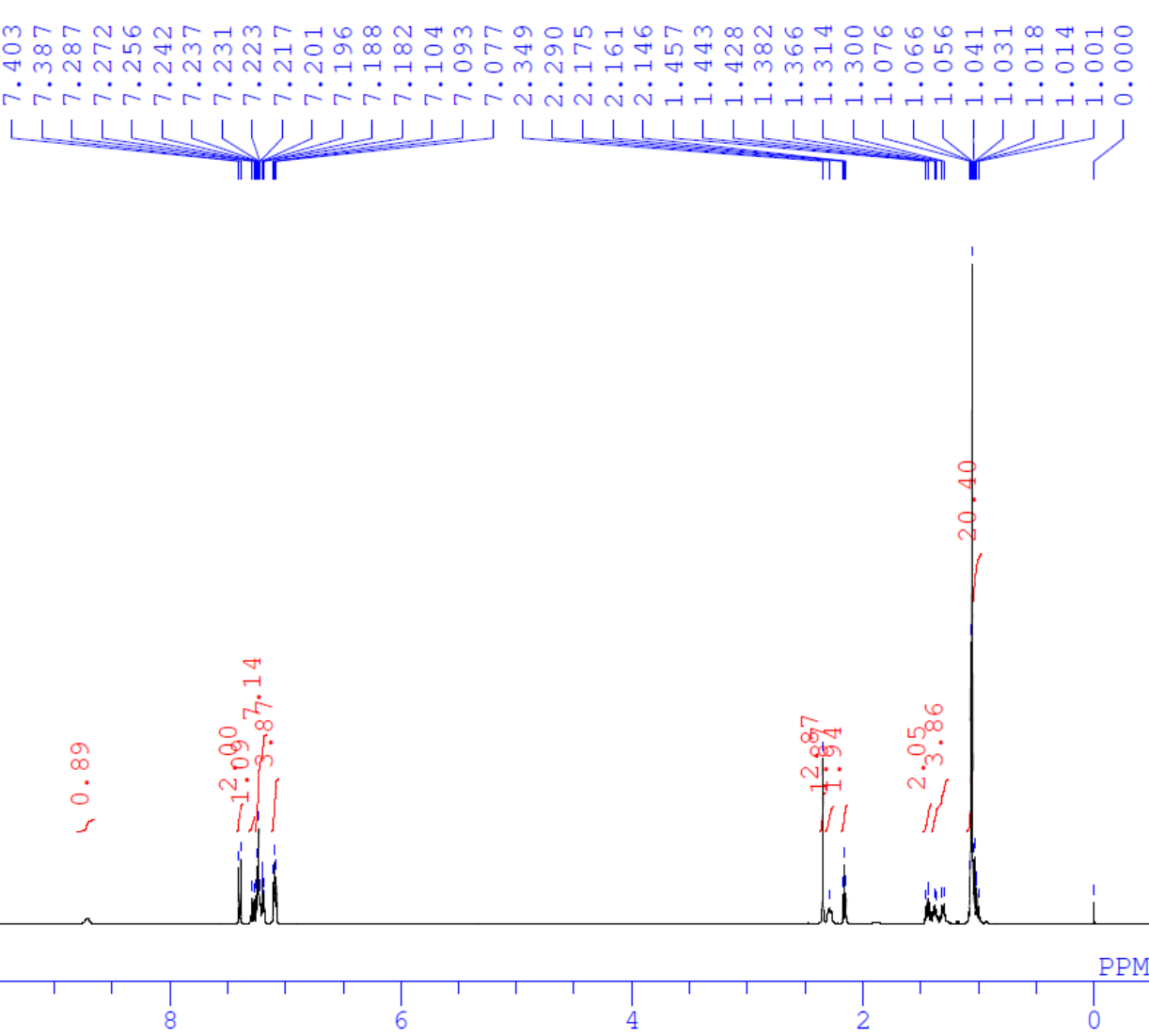

OBSET

OBEIN

POINT

FREQU

SCANS

ACQTM

PD

PW1

IRNUC

CTEMP

SLVNT

EXREF

$\mathrm{BE}$

RGAIN

IRNUC

CTEMP

SLVNT

EXREF

$B F$

RGAIN ym_06_024_3-1-1.al ingle pulse

2014-099-08 00:23:36 $1 \mathrm{H}$ proton.jxp

$500.16 \mathrm{MHz}$

$2.41 \mathrm{KHz}$

$6.01 \mathrm{~Hz}$

13107

$7507.51 \mathrm{~Hz}$ 8

1. 7459 sec $5.0000 \mathrm{sec}$

$1 \mathrm{H}$

6.22 usec

CDCL 3

$18.6 \mathrm{C}$

$0.00 \mathrm{ppm}$

$0.10 \mathrm{~Hz}$ 26 $\begin{array}{llllll}y m & 06 & 024 & 3 & 13 \mathrm{C}-1-\end{array}$ single pulse decoup 2014-09-08 00:25:06 $13 \mathrm{C}$

carbon. jxp

$125.77 \mathrm{MHz}$

$7.87 \mathrm{KHz}$

4. $21 \mathrm{~Hz}$

26214

$31446.54 \mathrm{~Hz}$

4096

0.8336 sec

2.0000 sec

$1 \mathrm{H}$

3.12 usec

CDCL 3

$19.3 \mathrm{C}$

$77.00 \mathrm{ppm}$

$0.10 \mathrm{~Hz}$

58 
<smiles>[15NH]/C(=C(\O)Br)C(c1ccc(Cl)cc1)c1ccc(Cl)cc1</smiles>
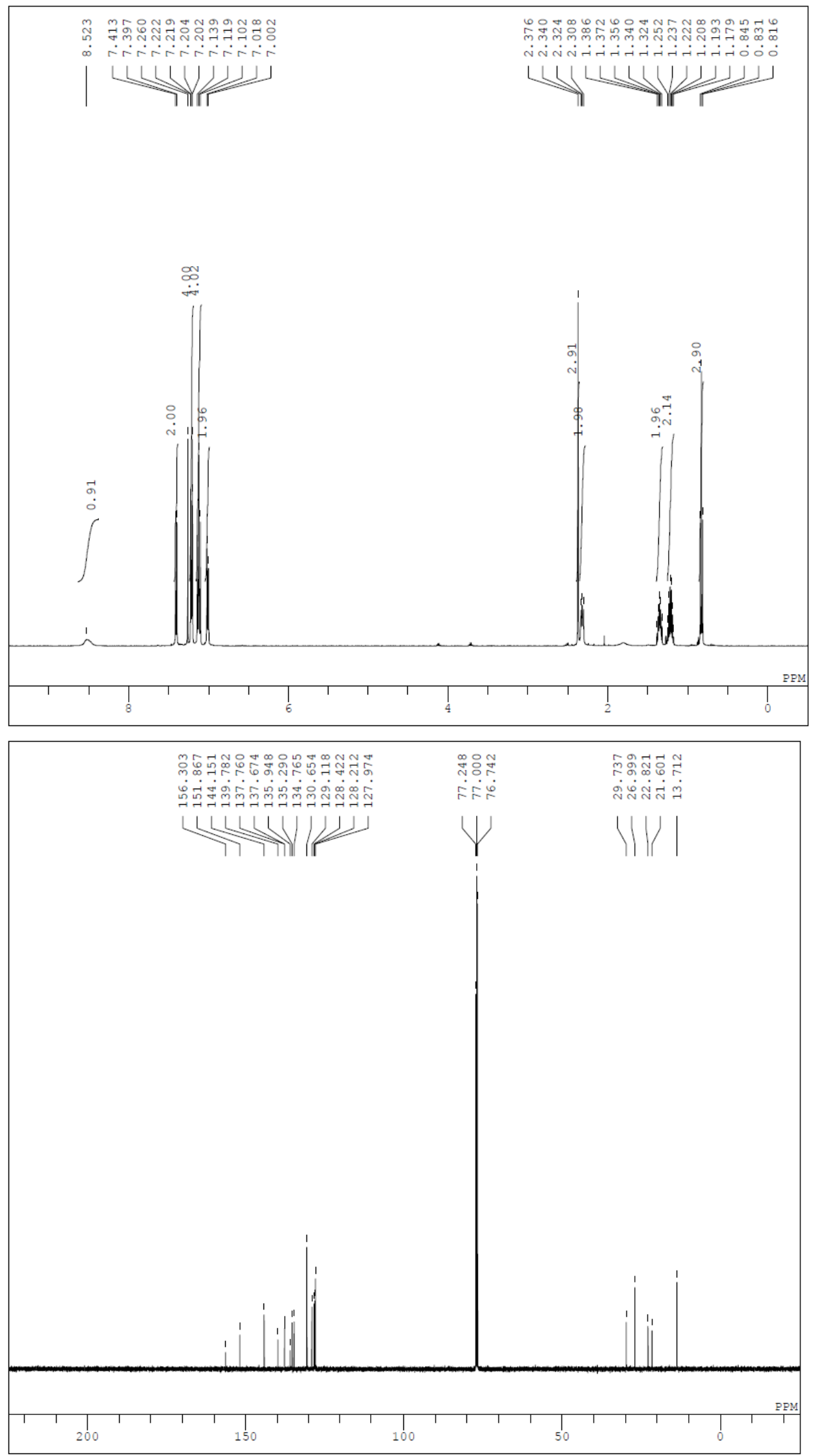

DFILE $\quad-$ Ym $-05 \_110 \_1$ 13C-1-1.als

DATIM 2013-11-22 10:23:38

OBNUC $13 \mathrm{C}$

EXMOD carbon, jxp

OBFRQ $\quad 125.77 \mathrm{MHz}$

OBSET $\quad 7.87 \mathrm{KHz}$

OBFIN $4.21 \mathrm{~Hz}$

POINT $\quad 26214$

FREQU 31446.5

ACQTM $\quad 0.8336 \mathrm{sec}$

PD 2.0000 sec

PW1 3.12 used

IRNUC $1 \mathrm{H}$

CTEMP $19.0 \mathrm{C}$

SIVNT CDCL 3

EXREF

$\begin{array}{lr}B F & 0.10 \mathrm{HZ} \\ \text { RGA.IN } & 58\end{array}$

$77.00 \mathrm{ppm}$
$0.10 \mathrm{~Hz}$ 
<smiles>COc1ccc(C(=C(C(=N)O)C(Br)=[Te])c2ccc(OC)cc2)cc1</smiles>
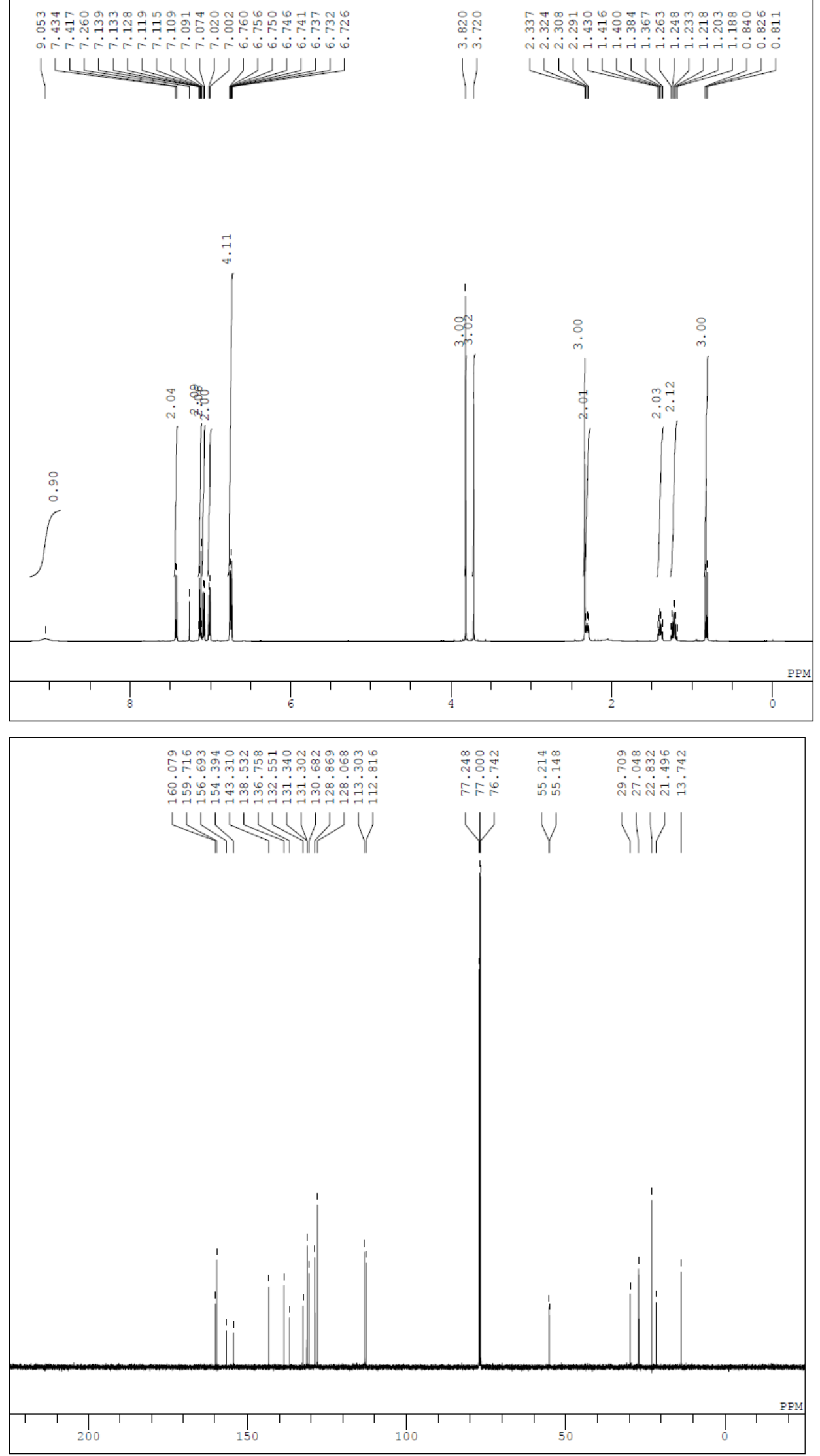


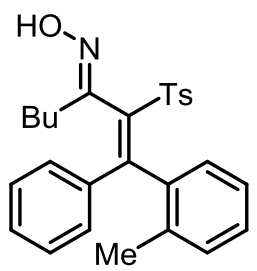

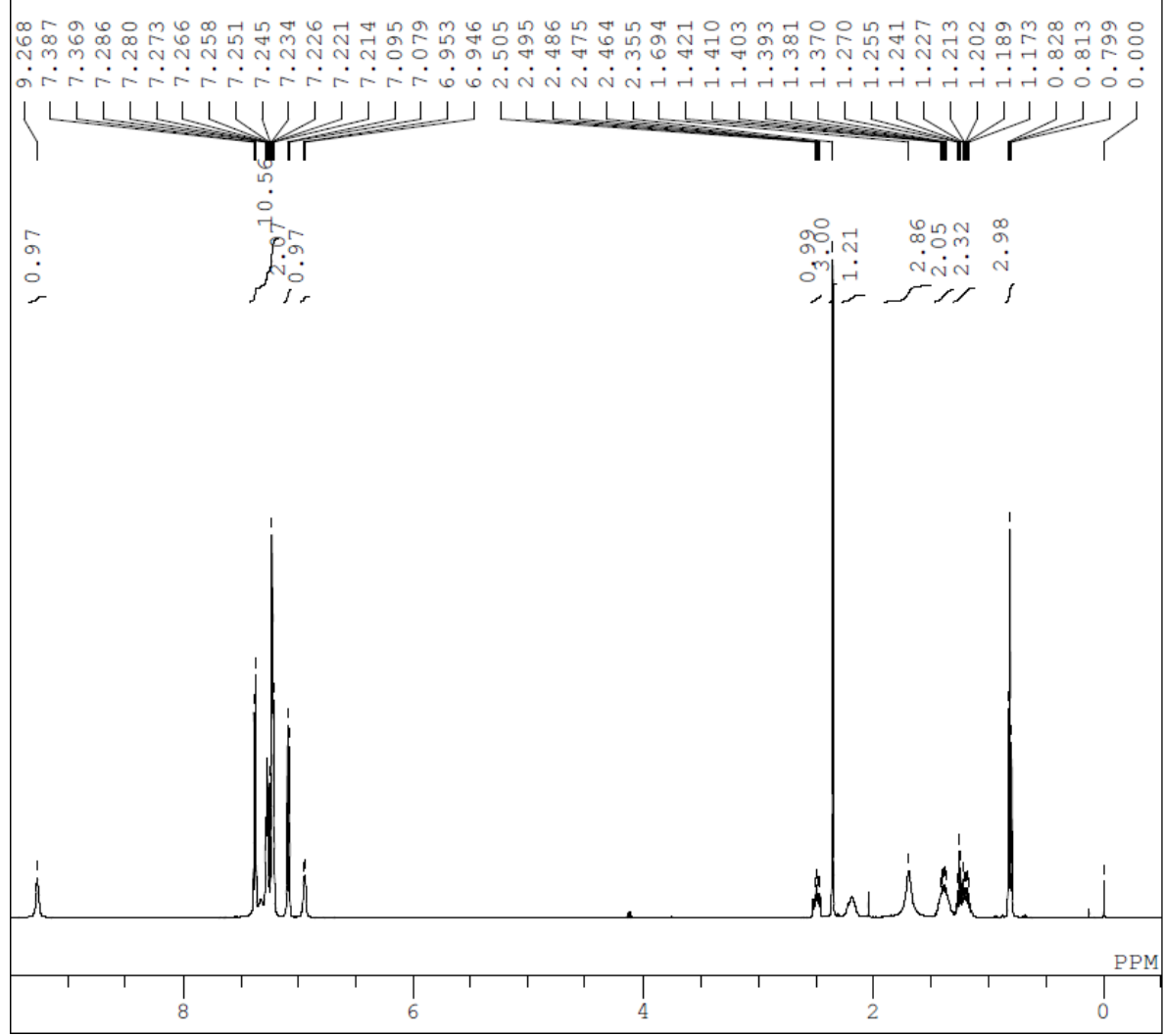

DFILE_ym_06_050_4-2-1.als

COMNT single_pulse

DATIM 2014-11-07 20:06:02

OBNUC $1 \mathrm{H}$

EXMOD proton.jxp

OBFRQ $\quad 500.16 \mathrm{MHz}$

OBSET $\quad 2.41 \mathrm{KHz}$

OBEIN $6.01 \mathrm{~Hz}$

POINT 13107

FREQU $\quad 7507.51 \mathrm{~Hz}$

SCANS 8

ACQTM $1.7459 \mathrm{sec}$

PD $5.0000 \mathrm{sec}$

PW1 6.22 usec

IRNUC $1 \mathrm{H}$

CTEMP $17.4 \mathrm{C}$

SLVNT CDCL3

$\begin{array}{ll}\text { EXREF } & 0.00 \mathrm{ppm} \\ \text { BE } & 0.10 \mathrm{~Hz}\end{array}$

RGAIN 26

DFILE Ym_06 050 4 13 C-1-1.als

COMNT single pulse decoupled gated

DATIM 2014-11-07 20:07:33

OBNUC 13C

EXMOD carbon, jXP

OBFRQ $\quad 125.77 \mathrm{MHz}$

$\begin{array}{lr}\text { OBSET } & 7.87 \mathrm{KHz}\end{array}$

OBFIN $4.21 \mathrm{HZ}$

OBFIN

POINT

FREQU

SCANS

ACQTM
PD
PW1
IRNUC

IRNUC

CTEMP

SLVNT

EXRE

BF
RGAIN

4.21
26214

$31446.54 \mathrm{~Hz}$

162
$0.8336 \mathrm{sec}$

$2.0000 \mathrm{sec}$

$1 \mathrm{H}$

3.12 usec

CDCL3

$17.5 \mathrm{c}$

$77.00 \mathrm{ppm}$

$0.10 \mathrm{~Hz}$

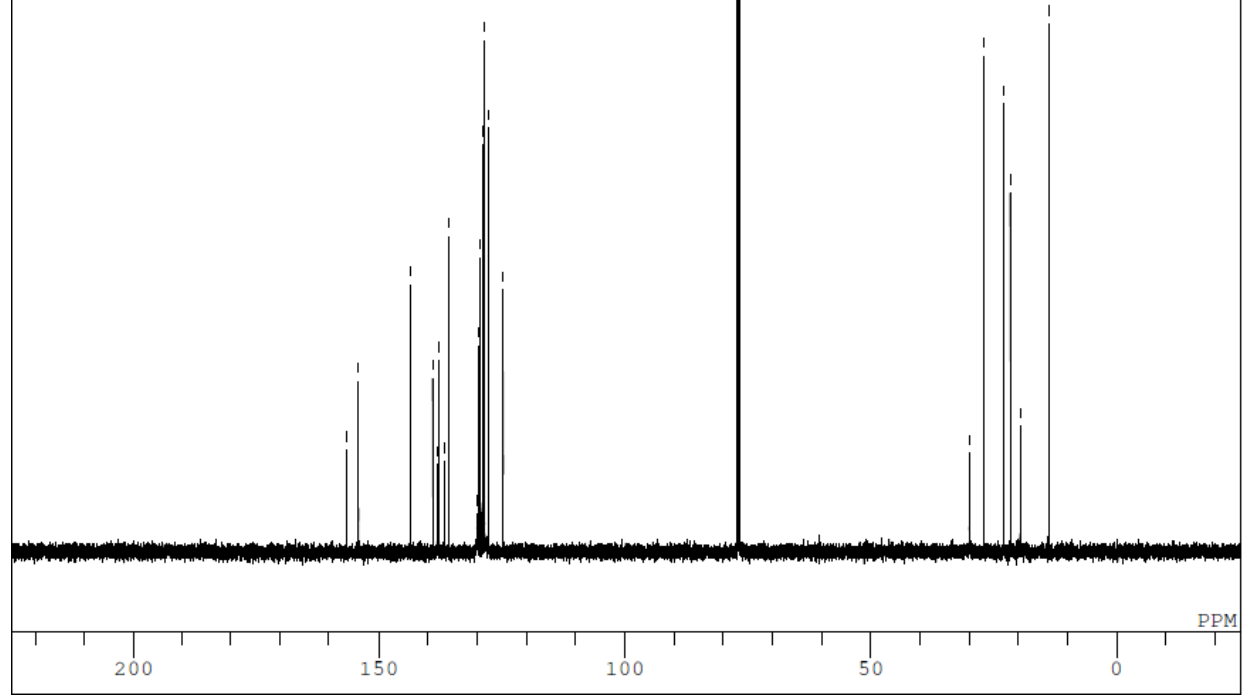


<smiles>Cc1ccccc1C(=C([Al])C(=N)O)c1ccccc1</smiles>
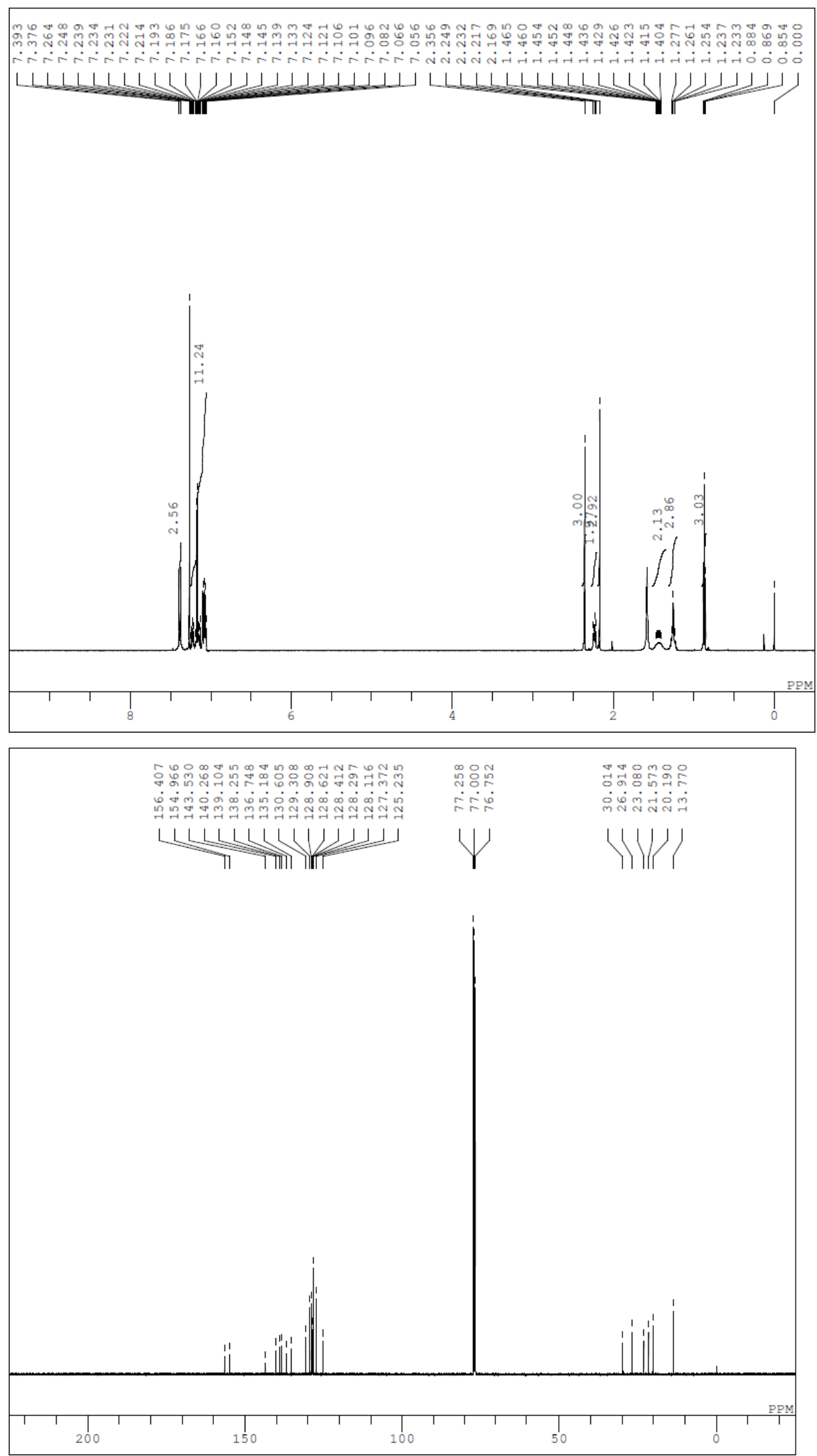

DFILE -YMm_06_050_5_13C-1-1.jdf

ExMOD carbon. jxp

$7.87 \mathrm{MHz}$

OBEIN

32767

FREQU $39308.18 \mathrm{~Hz}$

SCANS

PD

39308.18
8192

$0.8336 \mathrm{sec}$

2.0000 sec

3.12 usec

$\begin{array}{lll}\text { IRNUC } & 1 \mathrm{H} & \\ \text { CTEMP } & & 15.6 \mathrm{c}\end{array}$

SLVNT CDCL3

EXREF $\quad 77.00 \mathrm{ppm}$

BF $\quad 0.10 \mathrm{~Hz}$ 
<smiles>CCN(CC)C(=N)C(=N)c1ccccc1</smiles>

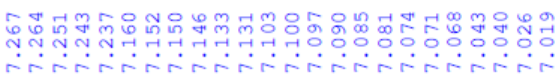

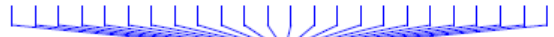

TII

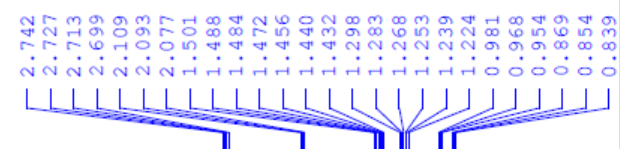

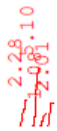
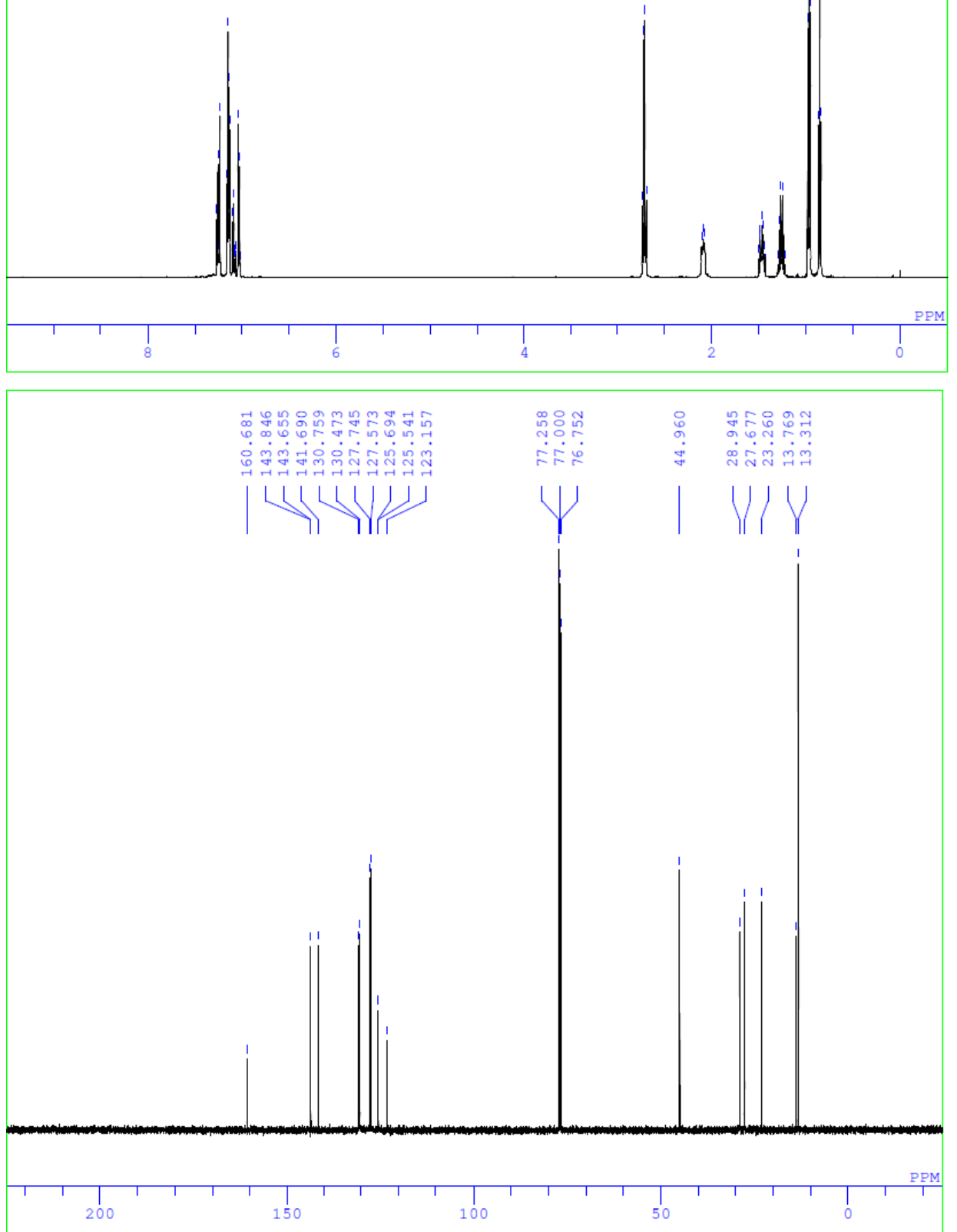

DF ILE

-ky_Et2N oxime s1-1-1.als

COMNT kY_Et $2 \mathrm{~N}$ oxime $\mathrm{s} 1$

DATIM 2014-09-15 22:17:09

EXMOD proton.jxp
OBFRQ

OBSET $2.41 \mathrm{KHz}$

OBFIN $\quad 6.01 \mathrm{~Hz}$

POINT 13107

FREQU $\quad 7507.51 \mathrm{~Hz}$

ACQTM

PD

PW1

IRNUC

CTEMP

SLVNT

EXRE

RGAIN

CDCL 3

$1.7459 \mathrm{sec}$

$5.0000 \mathrm{sec}$

6.22 usec

$18.7 \mathrm{c}$

$0.00 \mathrm{ppm}$

$0.10 \mathrm{~Hz}$

24
DFILE -ky_Et2N oxime_carbon-1-1.als COMNT ky Et $2 \mathrm{~N}$ Oxime

DATIM 2014-09-15 20:13:50

OBNUC $13 \mathrm{C}$

EXMOD carbon. jxp

$\begin{array}{lr}\text { OBFRQ } & 125.77 \mathrm{MHZ} \\ \text { OBSET } & 7.87 \mathrm{KHz}\end{array}$

OBFIN $4.21 \mathrm{~Hz}$

POINT 26214

FREQU $31446.54 \mathrm{~Hz}$

SCANS 1024

ACQTM $0.8336 \mathrm{sec}$

PD $2.0000 \mathrm{sec}$

PW1 2.0000 sec

IRNUC $1 \mathrm{H}$

CTEMP $19.0 \mathrm{C}$

SLVNT CDCL3

EXREF $\quad 77.00 \mathrm{ppm}$

$\begin{array}{lr}\text { BF } & 0.10 \mathrm{~Hz} \\ \text { RGAIN } & 56\end{array}$ 
<smiles>N=C(C(O)=C(c1ccccc1)c1ccccc1)C(=C(c1ccccc1)c1ccccc1)c1ccccc1</smiles>
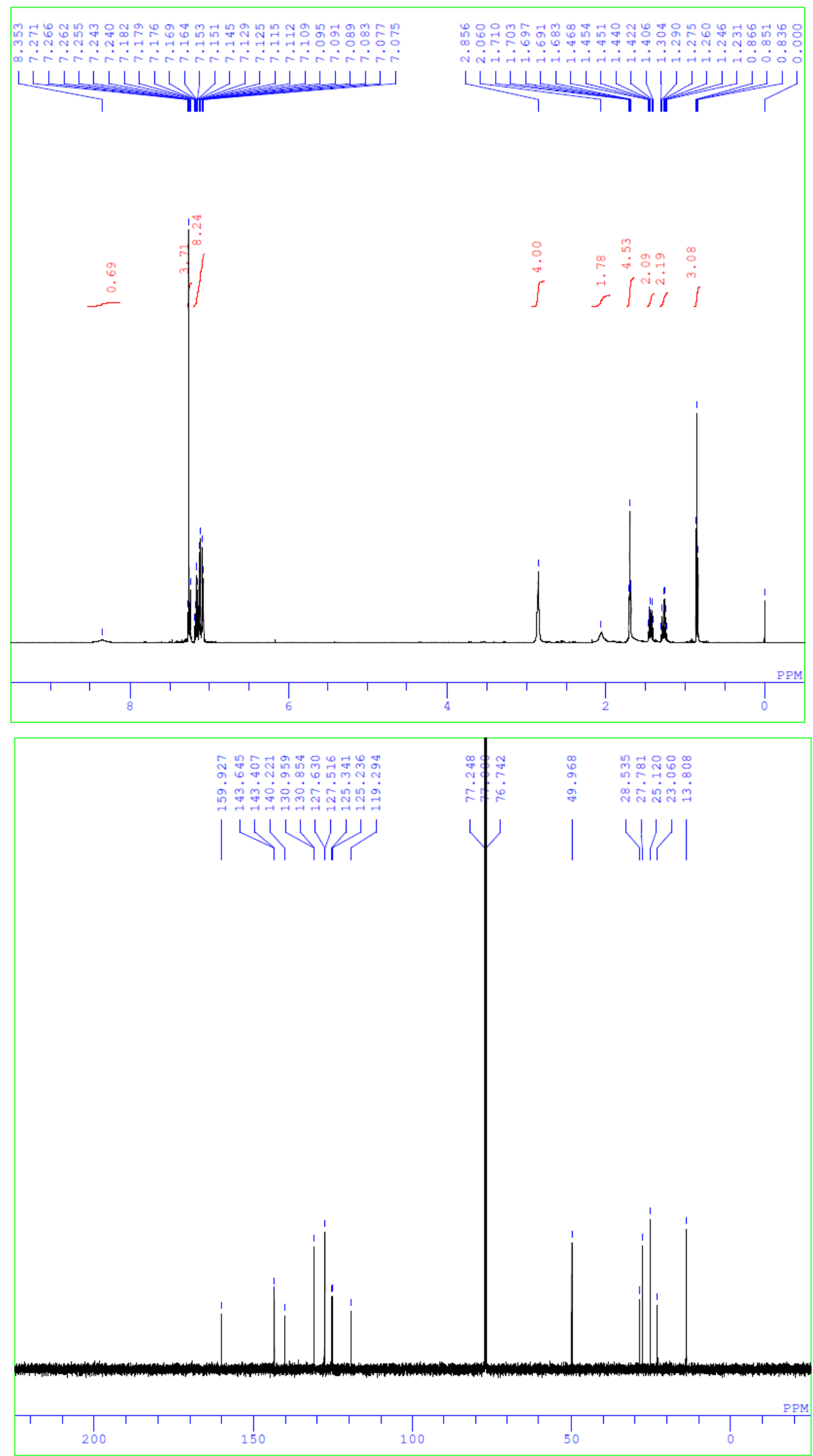

DFILE

ky_pyrrolidine oxime ssi-1-

Comi ky_pyrrolidine oxime ssi

EXMOD proton. jxp

OBSET

OBFIN

POINT

FREQ

ACQTM

PD

PW1

IRNUC 1

SLVNT CDCL

EXREF

RGAIN

CDCL3 $500.16 \mathrm{MHz}$ $2.41 \mathrm{KHz}$

$6.01 \mathrm{~Hz}$

$7507.51 \mathrm{~Hz}$

8

$1.7459 \mathrm{sec}$

$5.0000 \mathrm{sec}$

6.22 usec

$17.3 \mathrm{c}$

$0.00 \mathrm{ppm}$

$0.10 \mathrm{~Hz}$

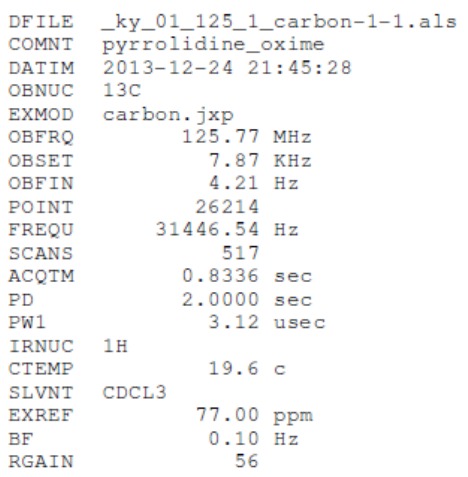


<smiles>O/N=C(C(=C(c1ccccc1)c1ccccc1)N1CCCCC1)/C(O)=C/c1ccccc1</smiles>

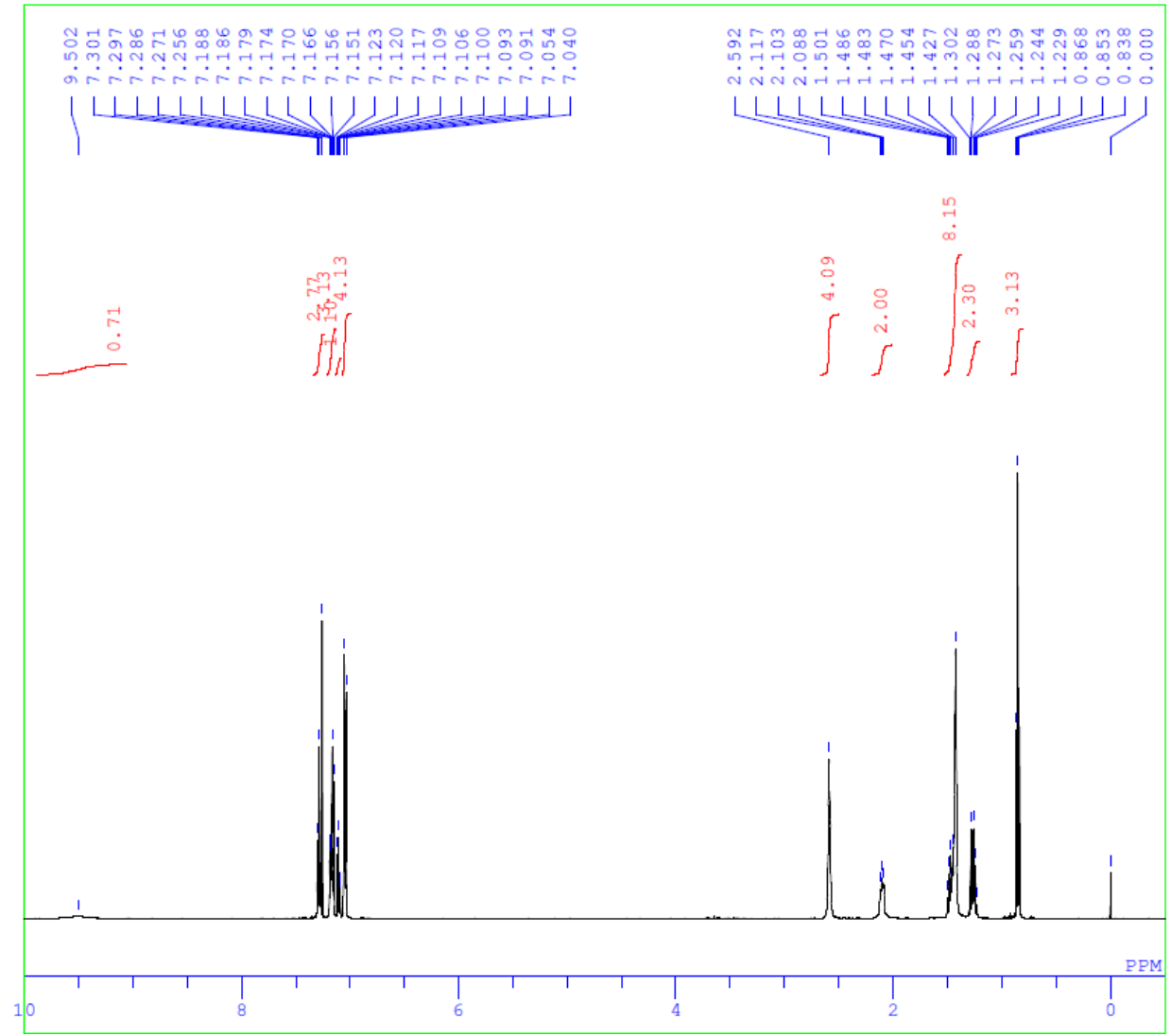

DFILE _ky_pipperidine oxime s1-1-1.

COMNT ky_pipperidine oxime s1

DATIM 2014-10-16 00:16:38

OBNUC $1 \mathrm{H}$

EXMOD proton. jxp

$\begin{array}{lr}\text { OBFRQ } & 500.16 \mathrm{MHZ} \\ \text { OBSET } & 2.41 \mathrm{KHz}\end{array}$

BBFIN $6.01 \mathrm{~Hz}$

$\begin{array}{lr}\text { POINT } & 13107\end{array}$

FREQU $\quad 7507.51 \mathrm{~Hz}$

8
SCANS
ACQTM

PDI

$5.0000 \mathrm{sec}$

6.22 usec

CTEMP $\mathrm{H} \quad 17.0 \mathrm{C}$

SLVNT CDCL3

EXREF $0.00 \mathrm{ppm}$

BF $\quad 0.10 \mathrm{~Hz}$

DFILE_ky_pipperidine oxime sl_car

COMNT ky pipperidine oxime s1

DATIM 2014-10-16 00:20:02

EXMOD carbon.jXp

OBFRQ $125.77 \mathrm{MHz}$

OBSET $\quad 7.87 \mathrm{KHZ}$

OBFIN $4.21 \mathrm{~Hz}$

POINT 26214

FREQU $\quad 31446.54 \mathrm{H}$

ACQTM $\quad 0.8336 \mathrm{sec}$

2. 0000 sec

PD1 $1.00 \mathrm{sec}$

IRNUC $1 \mathrm{H}$

CTEMP $17.3 \mathrm{C}$

SLVNT CDCL3

EXREF $\quad 77.00 \mathrm{ppm}$

$\mathrm{BF} \quad 0.10 \mathrm{~Hz}$

RGAIN $\quad 0.10 \mathrm{H}$

60
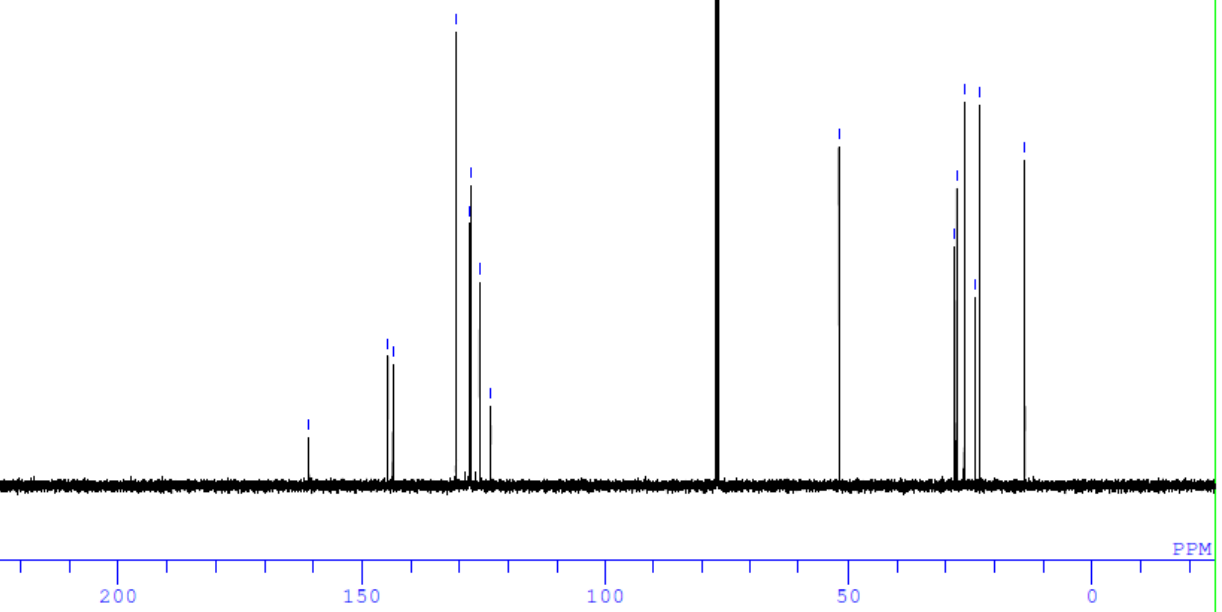
<smiles>O=C(CCCCCCC(=O)c1ccccc1)C(=C(c1ccccc1)c1ccccc1)N1CCOCC1</smiles>
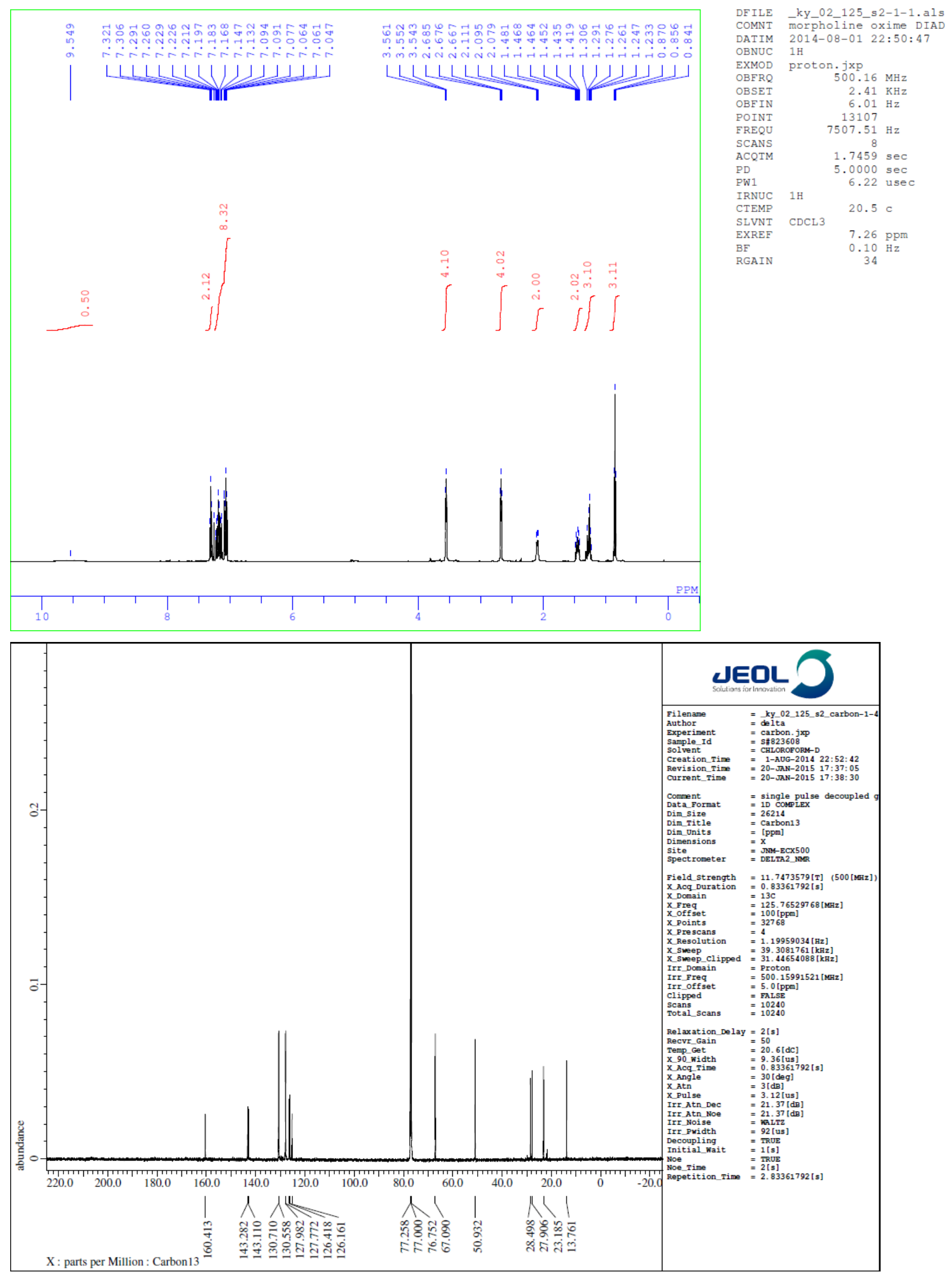
<smiles>CC(=O)O[C@H]1CCCN1C(=C(C(=N)O)c1ccccc1)c1ccccc1</smiles>
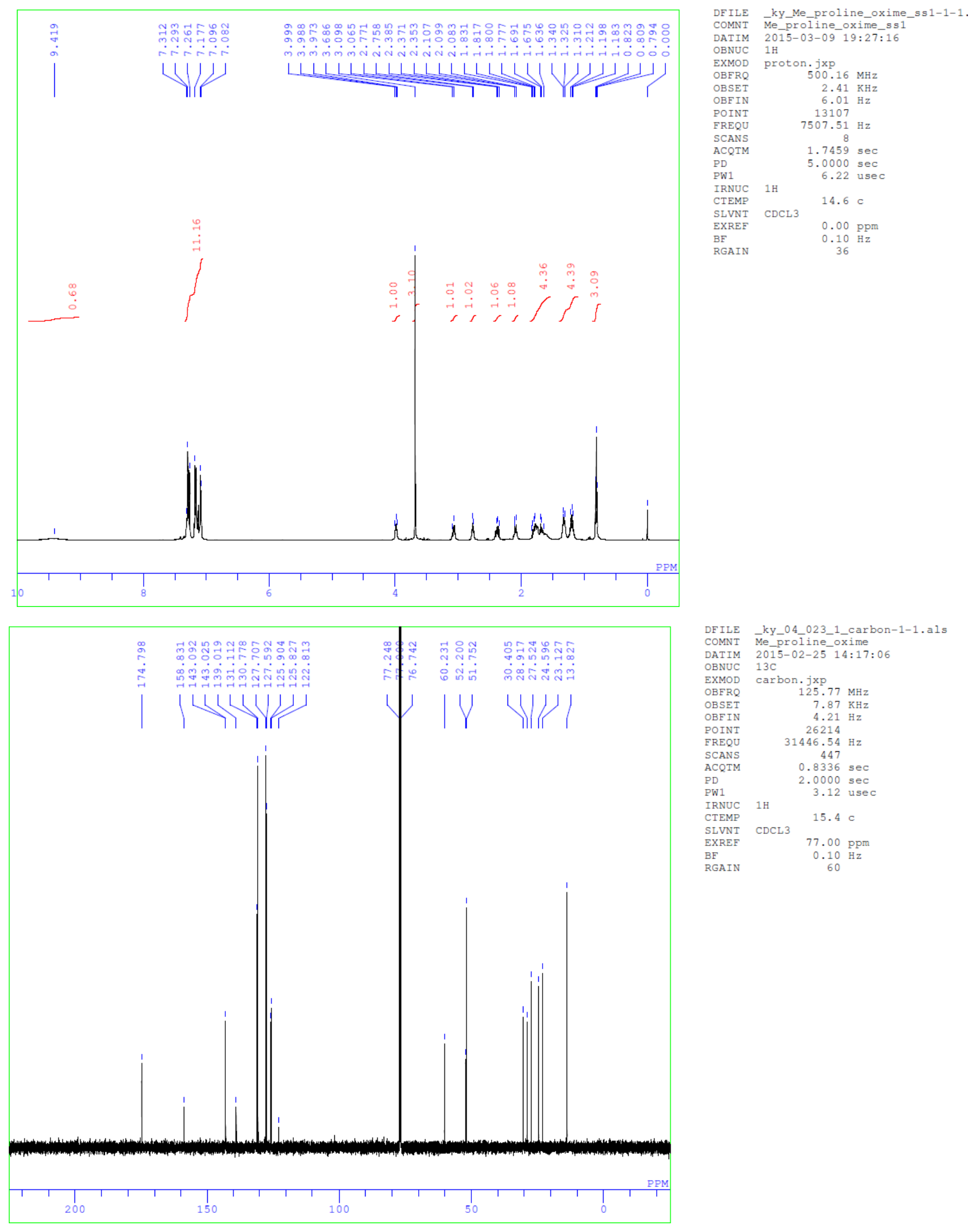
DFILE -ky_04_023_1_carbon-1-1.als
DATTM 2015-02-25 14:17:06
OBNUC $13 \mathrm{C}$
EXMOD carbon.jxp
OBFRQ $\quad 125.77 \mathrm{MH}$
OBSET $7.87 \mathrm{KHZ}$
POINT 26214
FREQU 31446.54 H
SCANS $\quad 447$
$\begin{array}{lr}\text { SCANS } & 447 \\ \text { ACQTM } & 0.8336\end{array}$
ACQT
PD 1
PW1 $2.0000 \mathrm{sec}$
IRNUC $1 \mathrm{H}$
CTEMP 15.4
SLVNT CDCL3
BGAIN
$77.00 \mathrm{ppm}$
$0.10 \mathrm{~Hz}$


<smiles>OC(Br)C(N[15F])=C(c1ccccc1)c1ccccc1</smiles>

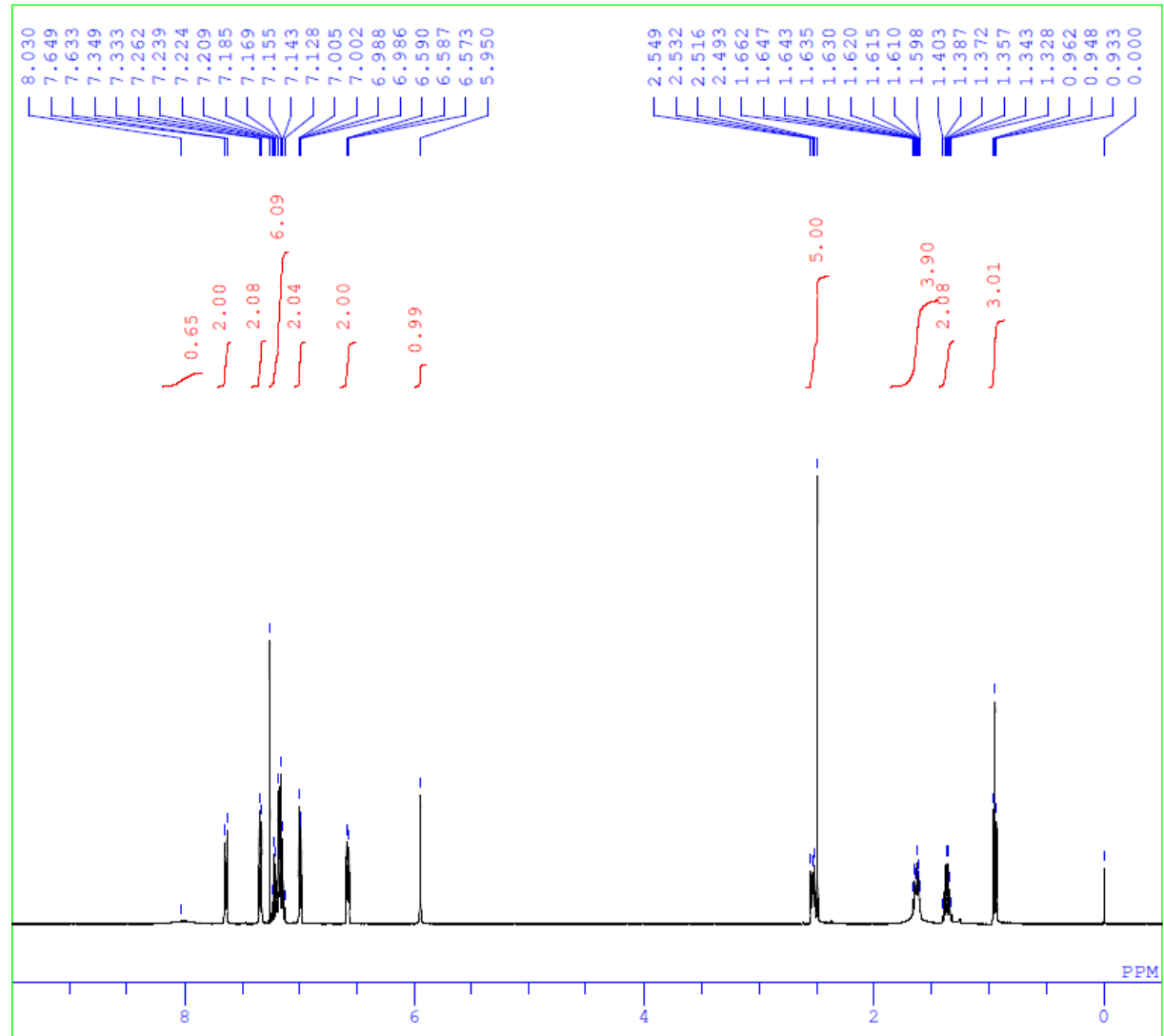

DFILE_ky_NHTs oxime-1-1.als

COMNT ky_NHTs oxime

DATIM 2014-10-22 01:15:10

OBNUC 1

EXMOD proton.jxp

\begin{tabular}{lr} 
OBFRQ & $500.16 \mathrm{MHz}$ \\
\hline & $2.41 \mathrm{KHz}$
\end{tabular}

OBFIN $\quad 6.01 \mathrm{~Hz}$

$\begin{array}{lr}\text { POINT } & 13107\end{array}$

FREQU $\quad 7507.51 \mathrm{~Hz}$

8
SCANS
$1.7459 \mathrm{sed}$

ACQTM

PD

$5.0000 \mathrm{sec}$

6.22 usec

$\begin{array}{lll}\text { IRNUC } & 1 \mathrm{H} & 18.0\end{array}$

SLVNT CDCL3

EXREF

EXR

RGAIN

$0.00 \mathrm{ppm}$

$0.10 \mathrm{~Hz}$

DFILE -ky_03_081_s1_carbon-1-1.als

COMNT TSNH2 OXIme DMEAD

DATIM 2014-10-20 21:13:52

OBNUC $13 \mathrm{C}$

EXMOD carbon.jXp

OBFRQ $\quad 125.77 \mathrm{MHz}$

OBSET $\quad 7.87 \mathrm{KHz}$

OBEIN $4.21 \mathrm{~Hz}$

POINT 26214

FREQU $\quad 31446.54 \mathrm{~Hz}$

$\begin{array}{lr}\text { SCANS } & 216 \\ \text { ACQTM } & 0.8336\end{array}$

2.0000 sec

PD1 3.12 sec

IRNUC $1 \mathrm{H}$

SLVNT CDCL3

EXREF

$\mathrm{BF}$

$17.6 \mathrm{c}$

$77.00 \mathrm{ppm}$

RGAIN $0.10 \mathrm{~Hz}$

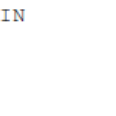

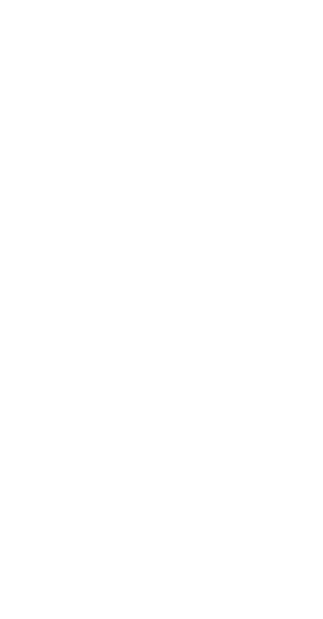


<smiles>N=C(C(=C(Br)CBr)c1ccccc1)N1C(=O)c2ccccc2C1=O</smiles>

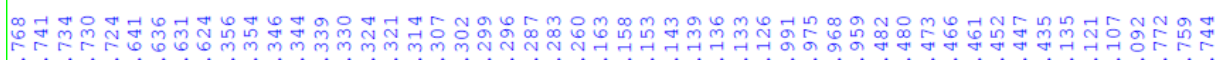
r

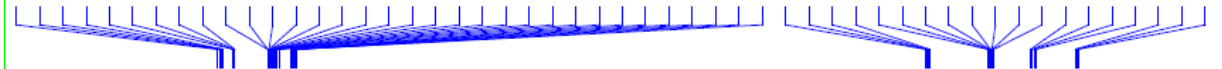
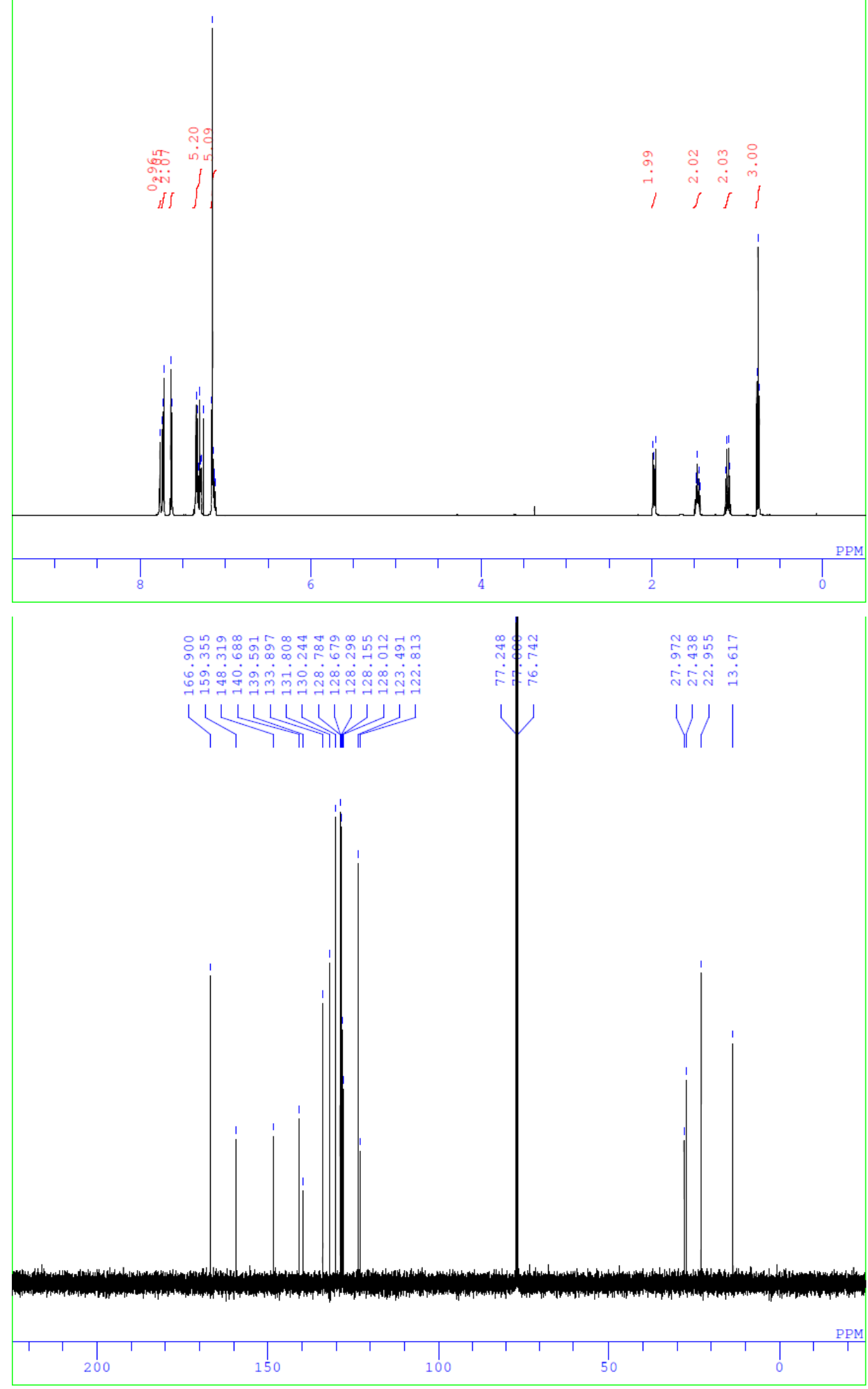

DFILE_TS_03_126_GPC_H-1-1.als

COMNT phthalimide

DATIM 2015-12-08 13:38:4

OBNUC 1

EXMOD proton.jxp

OBFRQ $\quad 500.16 \mathrm{MHz}$

OBSET $\quad 2.41 \mathrm{KHz}$

OBFIN $\quad 6.01 \mathrm{~Hz}$

POINT 13107

FREQU $\quad 7507.51 \mathrm{~Hz}$

SCANS

ACQTM

PD 1

IRNUC 1H

IRNUC

SLVNT CDCL3

EXREF

RGAIN

8
$1.7459 \mathrm{sec}$

$5.0000 \mathrm{sec}$

7.20 usec

$20.3 \mathrm{C}$

$7.26 \mathrm{ppm}$

$0.10 \mathrm{~Hz}$

DFILE_TS_03_126_GPC_C-1-1.als

COMNT phthalimide

DATIM 2015-12-08 13:40:07

OBNUC $13 \mathrm{C}$

EXMOD carbon.jxp

$\begin{array}{rr}\text { OBFRQ } & 125.77 \mathrm{MHz} \\ \text { OBSET } & 7.87 \mathrm{KHz}\end{array}$

OBFIN $4.87 \mathrm{KHZ}$

POINT 26214

FREQU $31446.54 \mathrm{~Hz}$

SCANS 0.353

353
ACQTM

$0.8336 \mathrm{sec}$

$\begin{array}{lrl}\text { PD } & 2.0000 \mathrm{sec} \\ \text { PW1 } & 4.00 \text { usec }\end{array}$

IRNUC $1 \mathrm{H}$

20.7

SLVNT CDCL3 77.00 prm

$\begin{array}{lr}\text { EXREF } & 77.00 \mathrm{ppm} \\ \text { BF } & 0.10 \mathrm{~Hz}\end{array}$ 

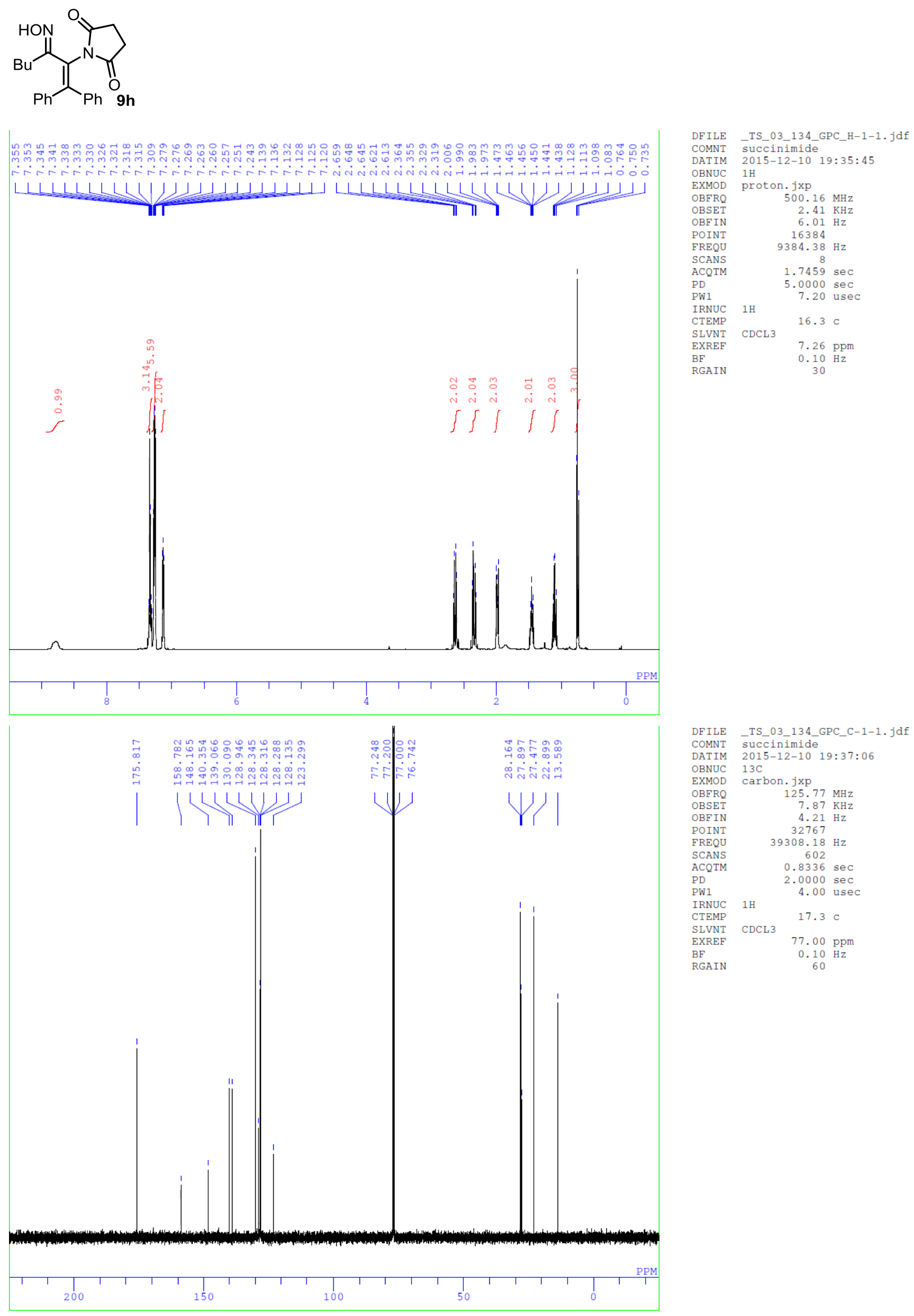
<smiles>O=[N+]([O-])C(=NO)C(=C(Br)c1ccccc1)c1ccccc1</smiles>

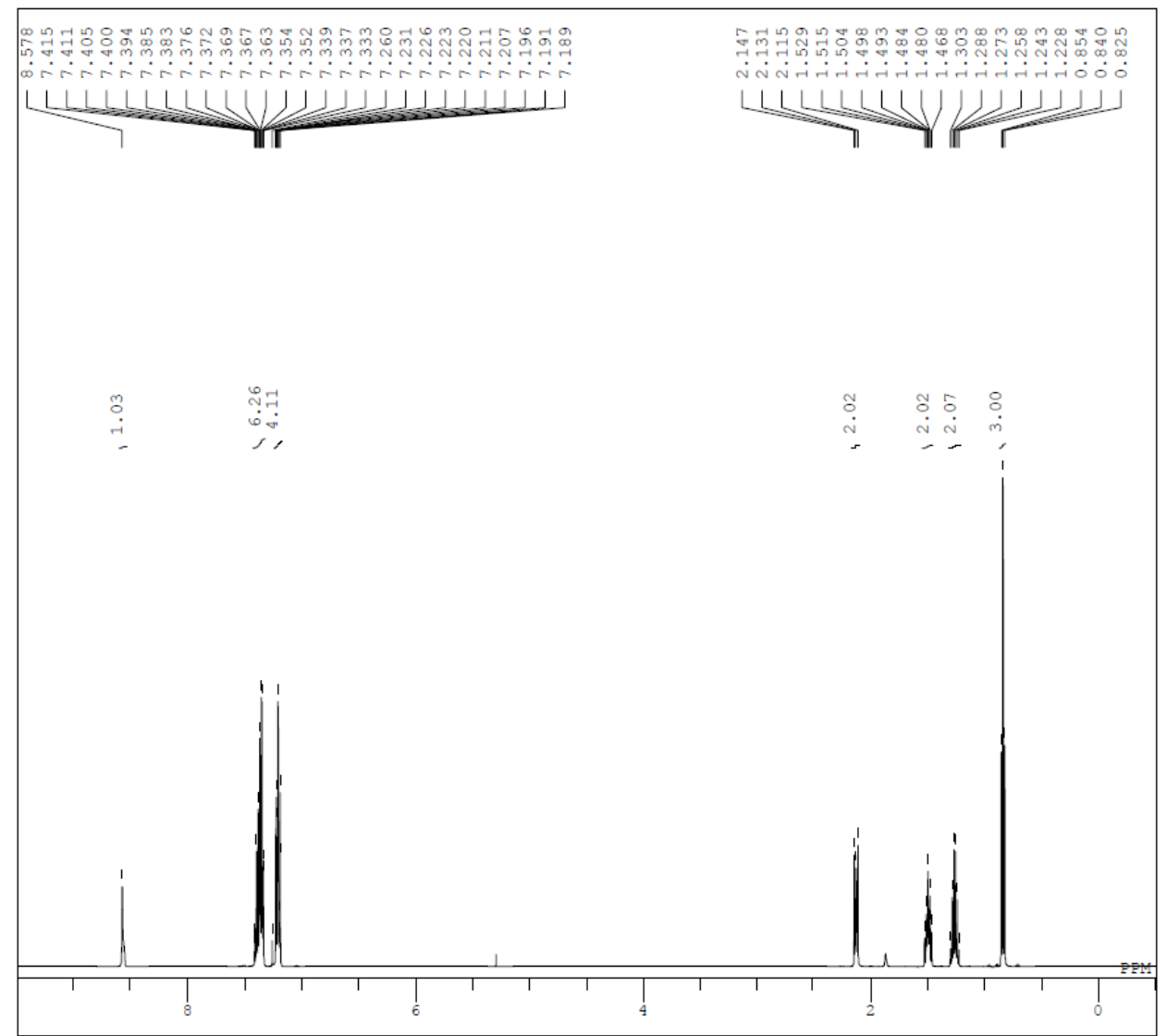

DFILE ky_nitro_oxime-1-1.als

OMNT nitro_oxime

DATM $2013-10-2311: 53: 38$

OBFRQ $\quad 500.16 \mathrm{MHz}$

OBSET $2.41 \mathrm{KHZ}$

$\begin{array}{ll}\text { OBFIN } & 6.01 \mathrm{~Hz} \\ \text { POINT } & 13107\end{array}$

FREQU $\quad 7507.51 \mathrm{~Hz}$

SCANS $\quad 1.7459 \mathrm{sec}$

PD 5.0000 sec

PD
PW1

IRNUC
CTEMP

SLVNT CDCL3

EXREF $\quad 7.26 \mathrm{ppm}$

$\begin{array}{lc}\text { BE } & 0.10 \mathrm{~Hz} \\ \text { RGAIN } & 30\end{array}$

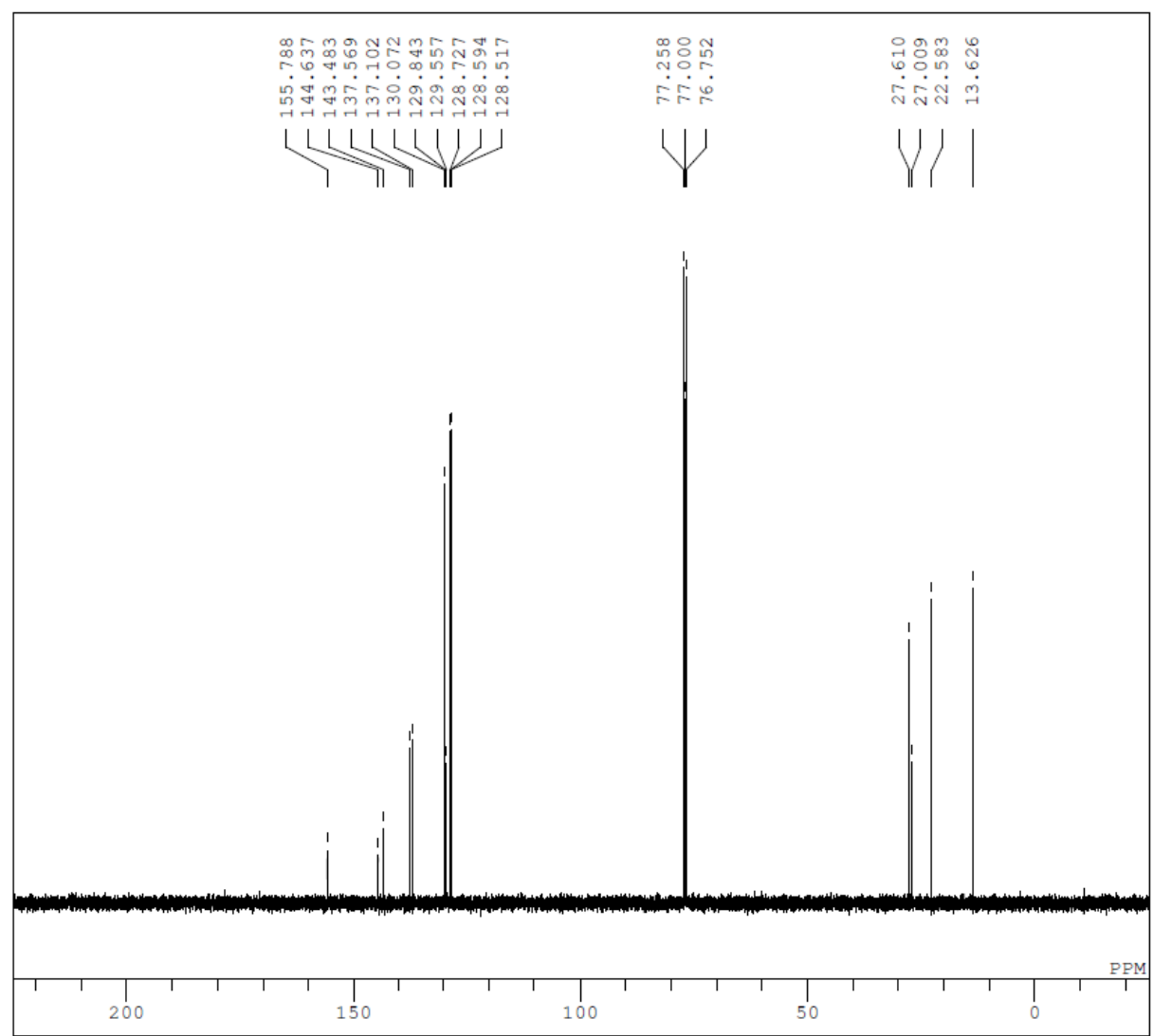

DFILE_ky_nitro_oxime_carbon-1-1

COMNT nitro oxime

DATIM 2013-10-23 11:55:04

OBNUC $13 \mathrm{C}$

OBFRQ Carbon. $j \mathrm{XP}$

OBFRQ $7.77 \mathrm{MHZ}$

OBET

POINT

FREQU

SCANS

SCANS

PD

IRNUC $1 \mathrm{H}$

CTEMP

SLVNT CDCL 3

EXREF

RGAIN

$7.87 \mathrm{KHz}$

26214

$31446.54 \mathrm{~Hz}$

160
0.8336

$2.0000 \mathrm{sec}$

19.0 c

$19.0 \mathrm{c}$

$77.00 \mathrm{ppm}$

$0.10 \mathrm{~Hz}$ 
9j

$6.7: 1$ ('H NMR ratio) inseparable mixture with $9 \mathbf{9 j}^{\prime}$
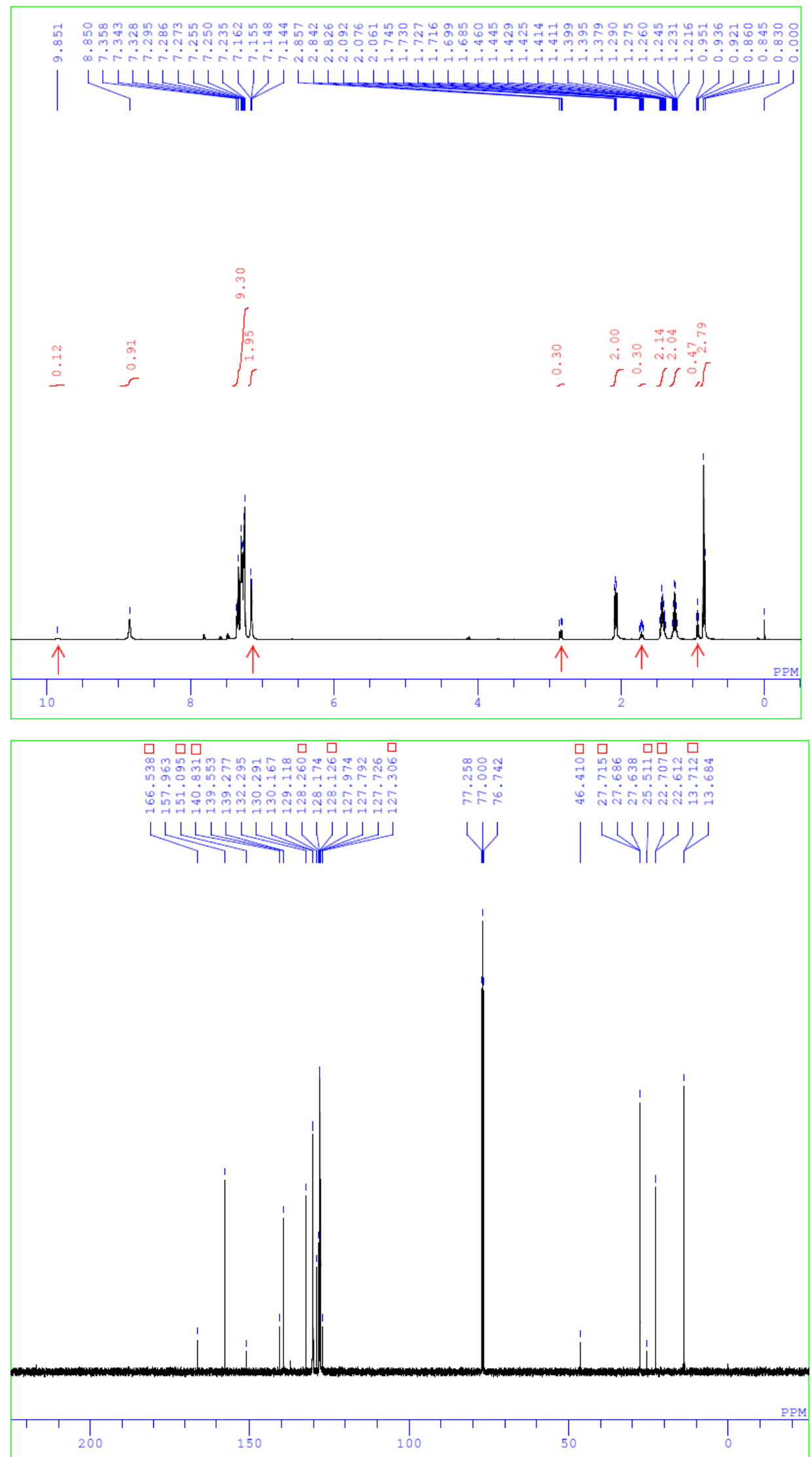

HO.

$\mathrm{Bu}$<smiles>PC1(c2ccccc2)N=C1CCl</smiles>

DFILE ky azide oxime ss1-1-1,als

ky_azide_oxime_ss1

OBNUC $1 \mathrm{H}$

OBFRQ $\quad 500.16 \mathrm{MHz}$

OBSET $\quad 2.41 \mathrm{KHZ}$

OBFIN $\quad 6.01 \mathrm{~Hz}$

13107
POINT

SCANS 8 .

ACQTM $\quad 1.7459 \mathrm{sec}$

PW1

IRNUC 1H 6.22 usec

CTEMP $1 \mathrm{H} \quad 15.9 \mathrm{C}$

SLVNT CDCL3 $3.00 \mathrm{pPm}$

$\begin{array}{ll}\text { EXREF } & 0.00 \mathrm{pp} \\ \mathrm{BF} & 0.10 \mathrm{~Hz}\end{array}$

$\begin{array}{ll}\text { BF } & 0.00 \text { PPIN } \\ \text { RGAIN } & 28\end{array}$

$\uparrow$ Azirine

DFILE _ky_azide_oxime_ss1_carbon-1

COMNT ky_azide_oxime_ssi

DATIM 2015-01-18 20:20:47

OBNUC $13 \mathrm{C}$

EXMOD carbon. jxp

OBFRQ $125,77 \mathrm{MHz}$

OBSET $\quad 7.87 \mathrm{KHz}$

OBFIN $\quad 4.21 \mathrm{~Hz}$

POINT 26214

FREQU 31446.54 Hz

$\begin{array}{ll}\text { SCANS } & 533 \\ \text { ACQTM } & 0.8336 \mathrm{sec}\end{array}$

PD 2.0000 sec

PD1 $2.0000 \mathrm{sec}$

IRNUC $1 \mathrm{H}$

CTEMP $16.3 \mathrm{C}$

$\begin{array}{lll}\text { SLVNT CDCL3 } & 77.00 \mathrm{ppm} \\ \text { EXREF } & & 0.10 \mathrm{mz}\end{array}$

$\begin{array}{lr}\text { EXREF } & 77.00 \mathrm{ppm} \\ \text { BF } & 0.10 \mathrm{~Hz}\end{array}$

$\square$ Azirine 
<smiles></smiles>

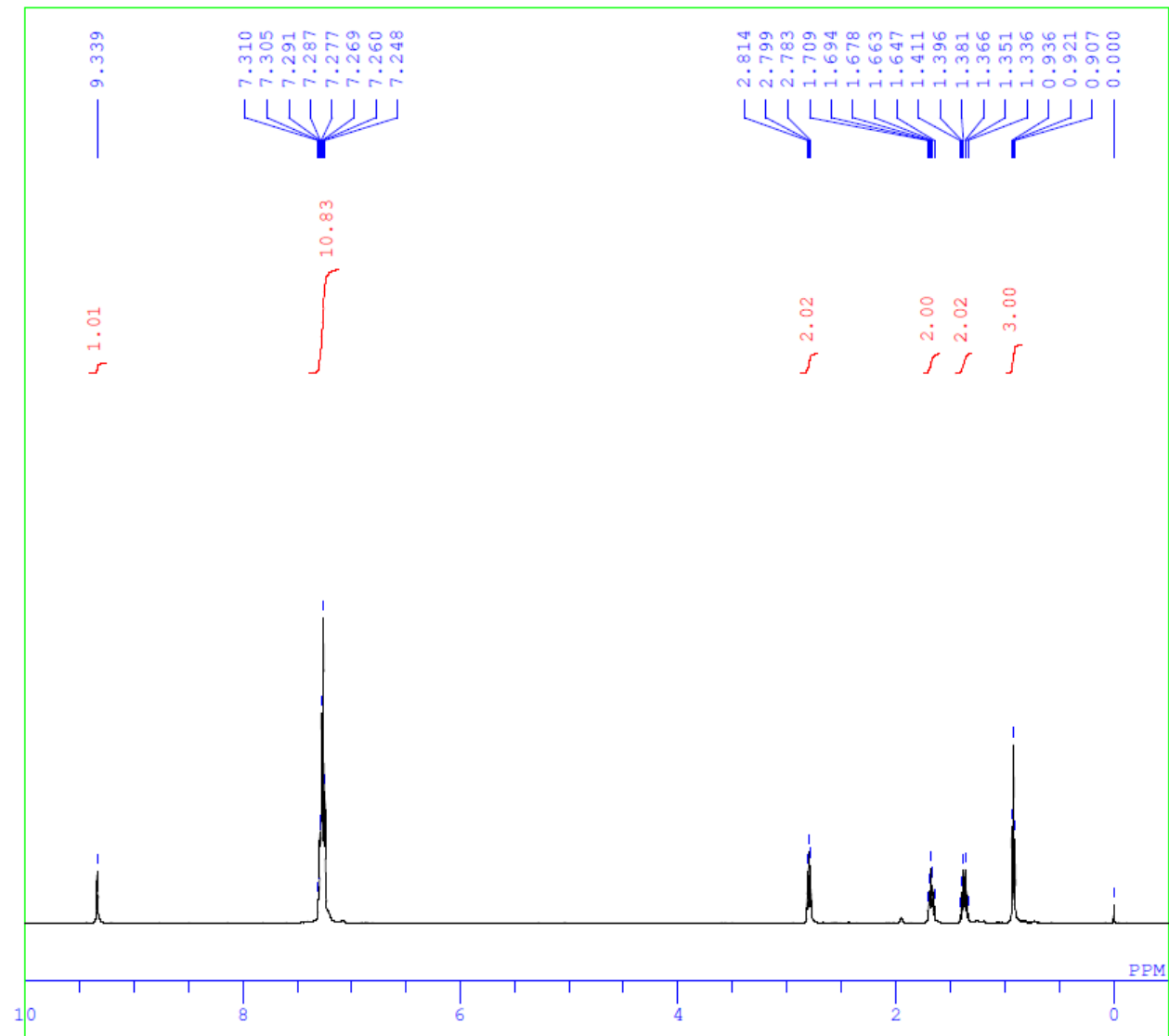

DFILE_ky_azirine_ss1-1-1.jdf

DATIM 2015-02-03 13:13:29

OBNUC 1

EXMOD proton. jxp
OBFRQ
$500.16 \mathrm{MHz}$

$\begin{array}{rr}\text { OBFRQ } & 500.16 \mathrm{MHZ} \\ \text { OBSET } & 2.41 \mathrm{KHZ}\end{array}$

DBFIN $\quad 6.01 \mathrm{~Hz}$

POINT 16384

FREQU $\quad 9384.38 \mathrm{~Hz}$

SCANS

ACQTM

PD

8
$1.7459 \mathrm{sec}$

$1.7459 \mathrm{sec}$
$5.0000 \mathrm{sec}$

PW1 $\quad 6.22$ usec

IRNUC $1 \mathrm{H}$

CTEMP $16.6 \mathrm{c}$

SLVNT CDCL3

EXREF

RGAIN

$0.00 \mathrm{ppm}$

$0.10 \mathrm{~Hz}$

DFILE -ky_azirine_ss1_carbon-1-1.jc

COMNT ky_azirine_ss 1

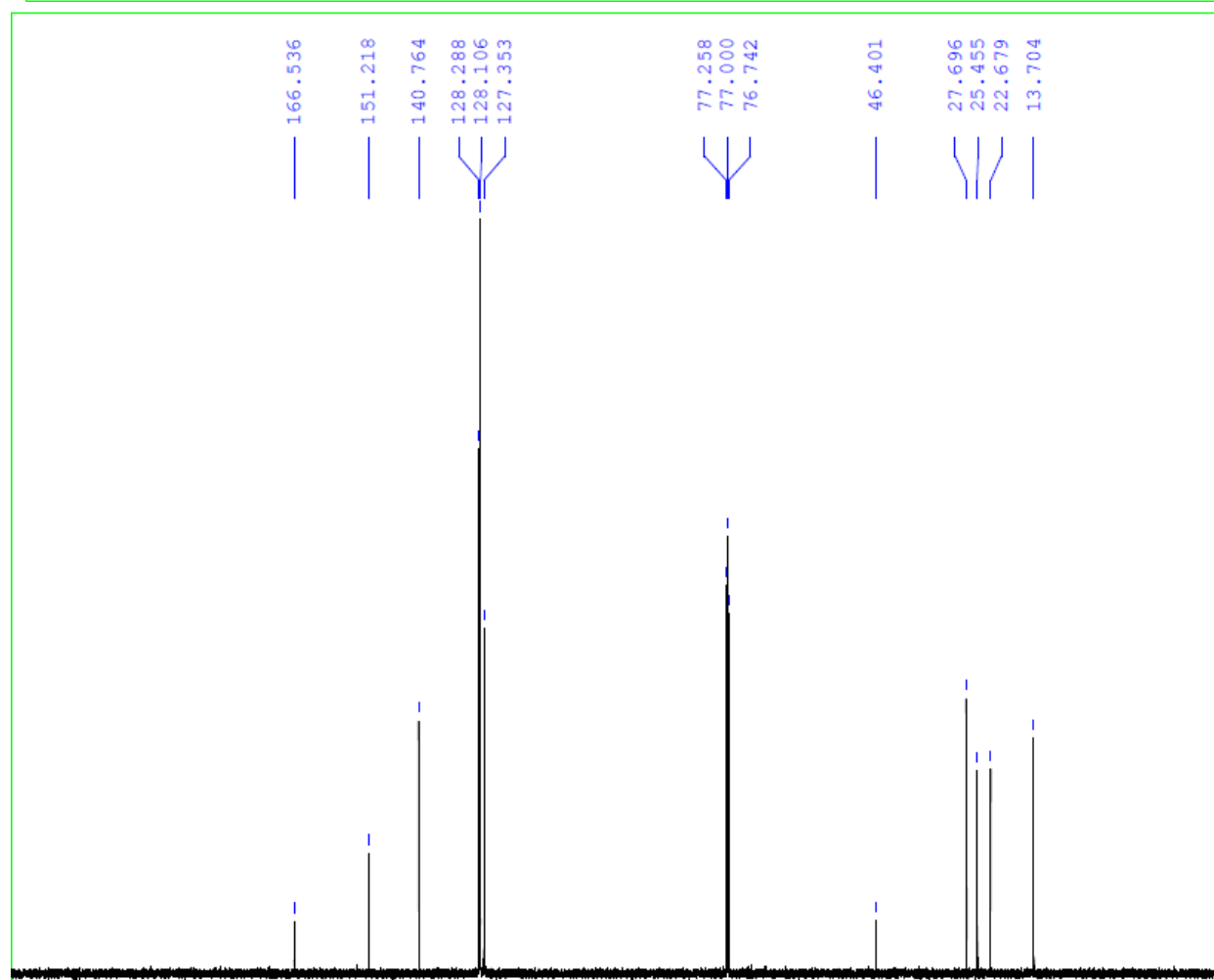

DATIM 2015-02-03 13:16:13

OBNUC $13 \mathrm{C}$

$\begin{array}{ll}\text { EXMOD } & \text { carbon. jXp } \\ \text { OBFRQ } & 125.77 \mathrm{MHz}\end{array}$

OBSET $\quad 7.87 \mathrm{KHz}$

OBFIN $\quad 4.21 \mathrm{~Hz}$

POINT

FREQU

SCANS

ACQTM

PD

PW1

IRNUC $1 \mathrm{H}$

CTEMP

SLVNT CDCL3

EXREF

$\mathrm{BF}$ 2767

$39308.18 \mathrm{~Hz}$ 384
.8336

$0.8336 \mathrm{sec}$

2.0000 sec
3.12 usec

RGAIN

$16.9 \mathrm{c}$

$77.00 \mathrm{ppm}$

$0.10 \mathrm{~Hz}$

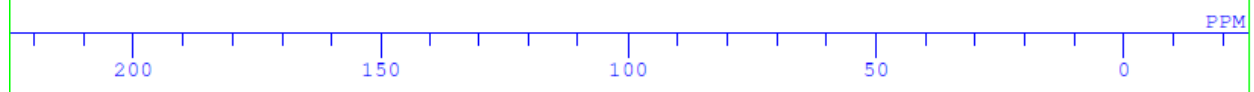


<smiles>O=C(Br)/C(=C(/F)c1ccccc1)c1ccccc1</smiles>

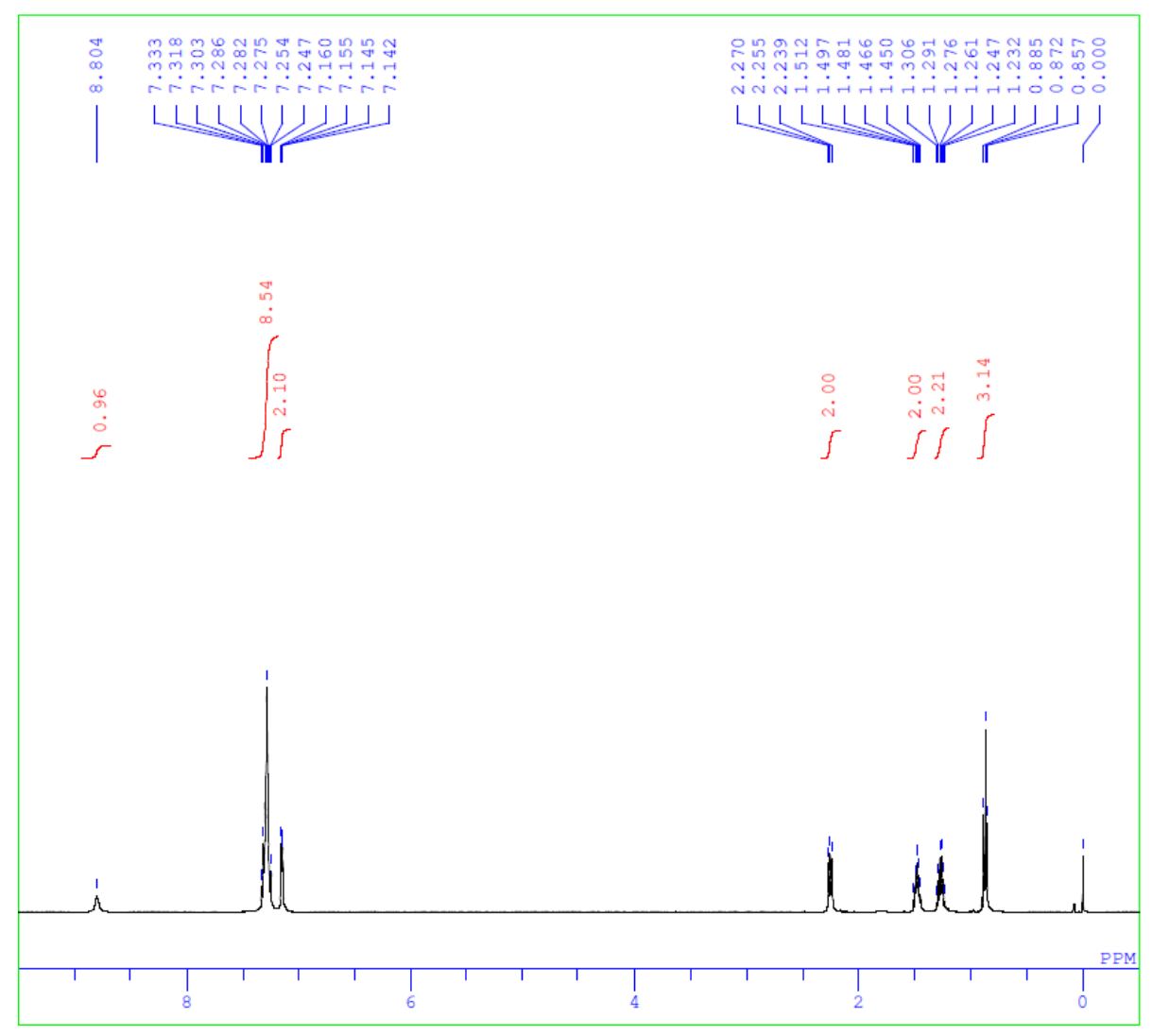

DFILE -ky_F oxime_s1-1-1.als

DATIM 2015-01-17 21:47:42

DBNUC 1 H

OBNuC 1H

EXMOD proton. jXP $16 \mathrm{MH}$

$\begin{array}{lr}\text { OBFRQ } & 500.16 \mathrm{MHz} \\ \text { OBSET } & 2.41 \mathrm{KHz}\end{array}$

$\begin{array}{ll}\text { OBSET } & 2.41 \mathrm{KHz} \\ \text { OBFIN } & 6.01 \mathrm{~Hz}\end{array}$

POINT 13107

FREQU $\quad 7507.51 \mathrm{~Hz}$

SCANS

PD

PD 1

PW1

CTEMP

SLVNT CDCL3

EXREF

BF

$1.7459 \mathrm{sec}$

$5.0000 \mathrm{sec}$

6.22 use

$12.9 \mathrm{c}$

RGAIN

$0.00 \mathrm{ppm}$

$0.10 \mathrm{~Hz}$

DFILE_ky_F oxime_s1_carbon-1-1.als

COMNT ky_F $\circ$

DATIM 2015-01-

OBNUC 130

$\begin{array}{lr}\text { EXMOD } & \text { carbon. jXp } \\ \text { OBFRQ } & 125.77 \mathrm{MHz}\end{array}$

OBSET $\quad 7.87 \mathrm{KH}$

DOINT

POINT

SCANS

ACQTM

PD

PW1

IRNUC

SLVNT

EXREF
BF

BF
RGAIN

$4.21 \mathrm{H}$

$39308.18 \mathrm{~Hz}$

$\begin{array}{rl}379 & \mathrm{~Hz} \\ 379 & \end{array}$

$0.8336 \mathrm{sec}$

$2.0000 \mathrm{sec}$

$.0000 \mathrm{sec}$
3.12 use

CDCL 3

$13.3 \mathrm{c}$

$77.00 \mathrm{ppm}$

$0.10 \mathrm{~Hz}$

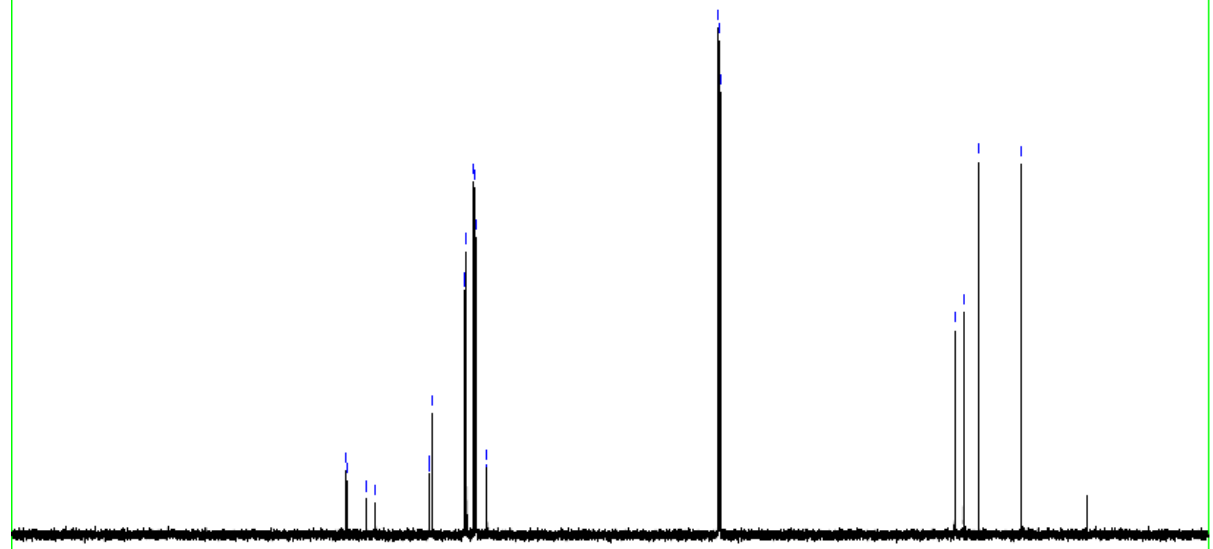

60

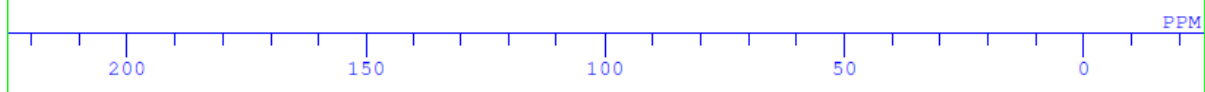


<smiles>O/N=C(/O)C(F)=C(P)P</smiles>

9k $\mathrm{Ph} \mathrm{Ph}_{19} \mathrm{~F}$ NMR with/without hexafluorobenzene $(\delta-162.0)$ as an internal standard reference.

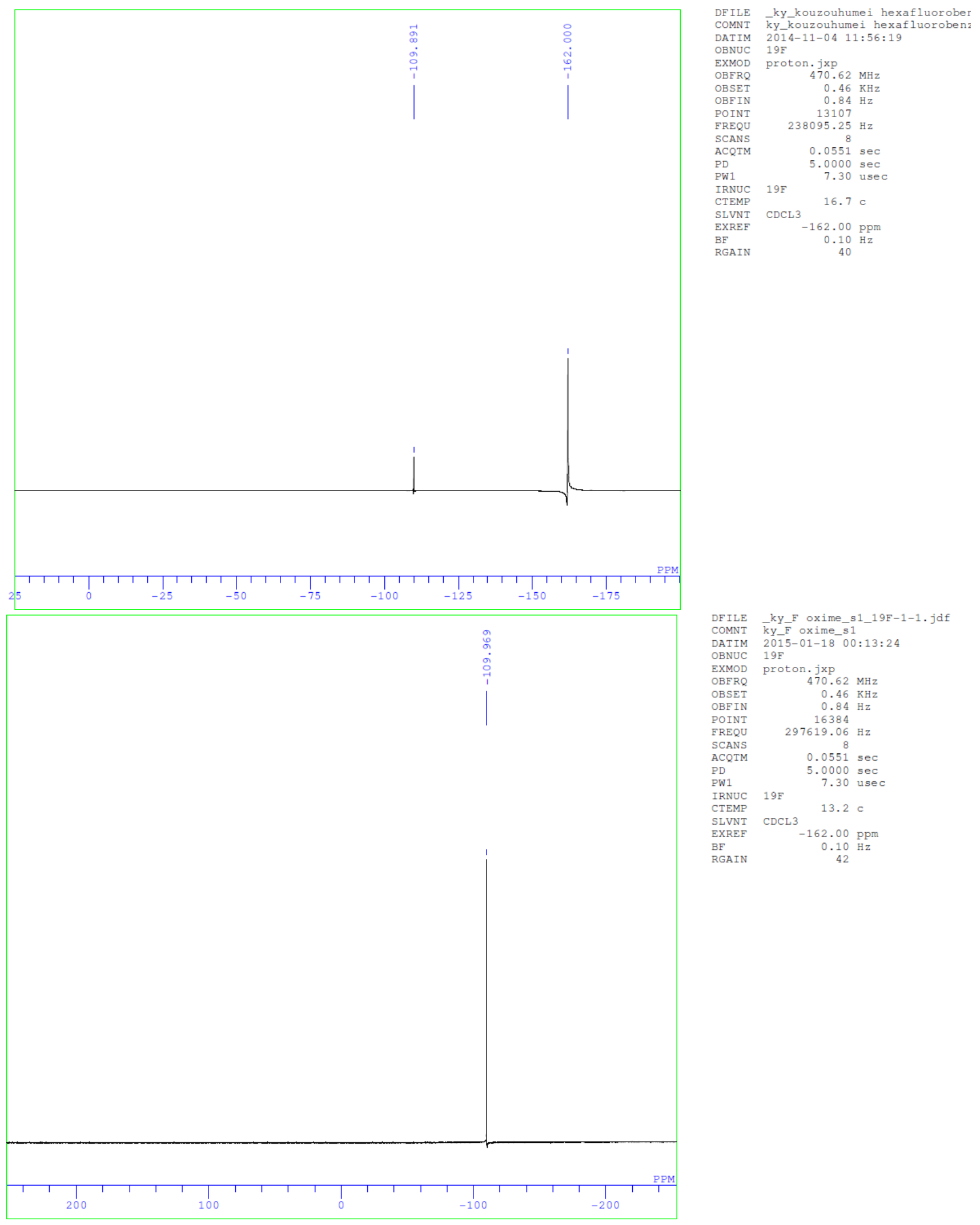


<smiles>COC(C(=N)O)=C(c1ccccc1)c1ccccc1</smiles>

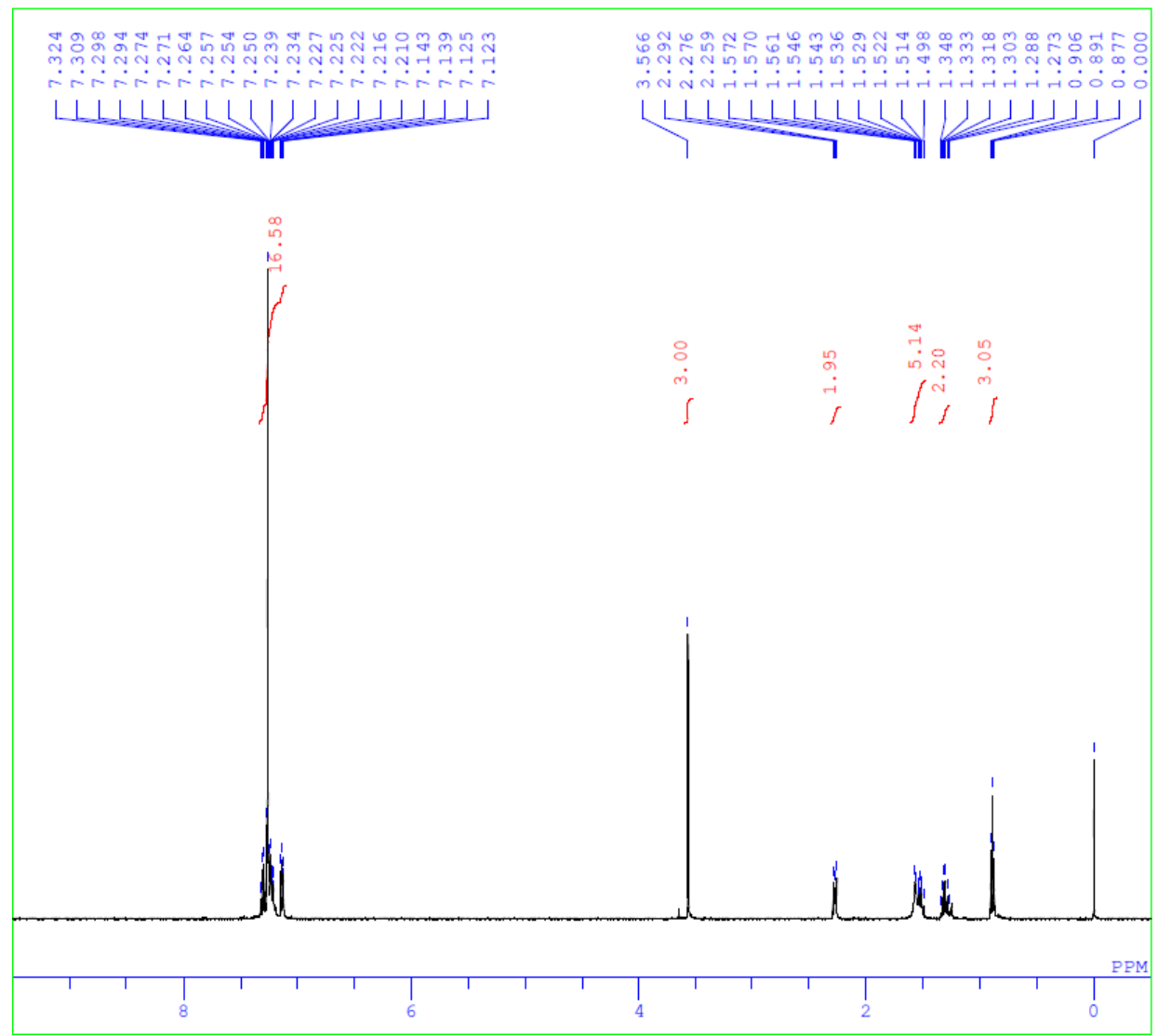

DFILE -ky_MeO oxime_re1-1-1.als

COMNT ky_MeO oxime_re1

DATIM 2015-01-27 00:21:52

OBNUC 1 H

EXMOD
OBFRQ

$\begin{array}{rr}\text { OBFRQ } & 500.16 \mathrm{MHz} \\ \text { OBSET } & 2.41 \mathrm{KHz}\end{array}$

OBFIN $\quad 6.01 \mathrm{~Hz}$

POINT 13107

FREQU $\quad 7507.51 \mathrm{~Hz}$

SCANS

ACQTM

PD

PW1

IRNUC $1 \mathrm{H}$

CTEMP

SLVNT CDCL 3

EXREF

BF

8
$1.7459 \mathrm{sec}$

$5.0000 \mathrm{sec}$

6.22 usec

RGAIN

$0.00 \mathrm{ppm}$

$0.10 \mathrm{~Hz}$

DFILE -ky_MeO_oxime_s1_carbon-1-1.

COMNT ky MeO oxime s1

DATIM 2015-01-24 22:35:03

OBNUC $13 \mathrm{C}$

EXMOD carbon. jxp

OBFRQ $\quad 125.77 \mathrm{MHz}$

OBSET $\quad 7.87 \mathrm{KHz}$

OBFIN $\quad 4.21 \mathrm{~Hz}$

POINT 26214

FREQU $\quad 31446.54 \mathrm{~Hz}$

SCANS 15000

ACQTM $\quad 0.8336 \mathrm{sec}$

PD 2.0000 sec

PW1 $2.0000 \mathrm{sec}$

IRNUC $1 \mathrm{H}$

CTEMP

SLVNT CDCL 3

EXREF

$\mathrm{BF}$

3.12 usec

$\begin{array}{lr}\text { EXREF } & 77.00 \mathrm{ppm} \\ \text { BF } & 0.10 \mathrm{~Hz} \\ \text { RGAIN } & 60\end{array}$

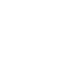


<smiles>CC(=O)OC(C(=N)O)=C(c1ccccc1)c1ccccc1</smiles>
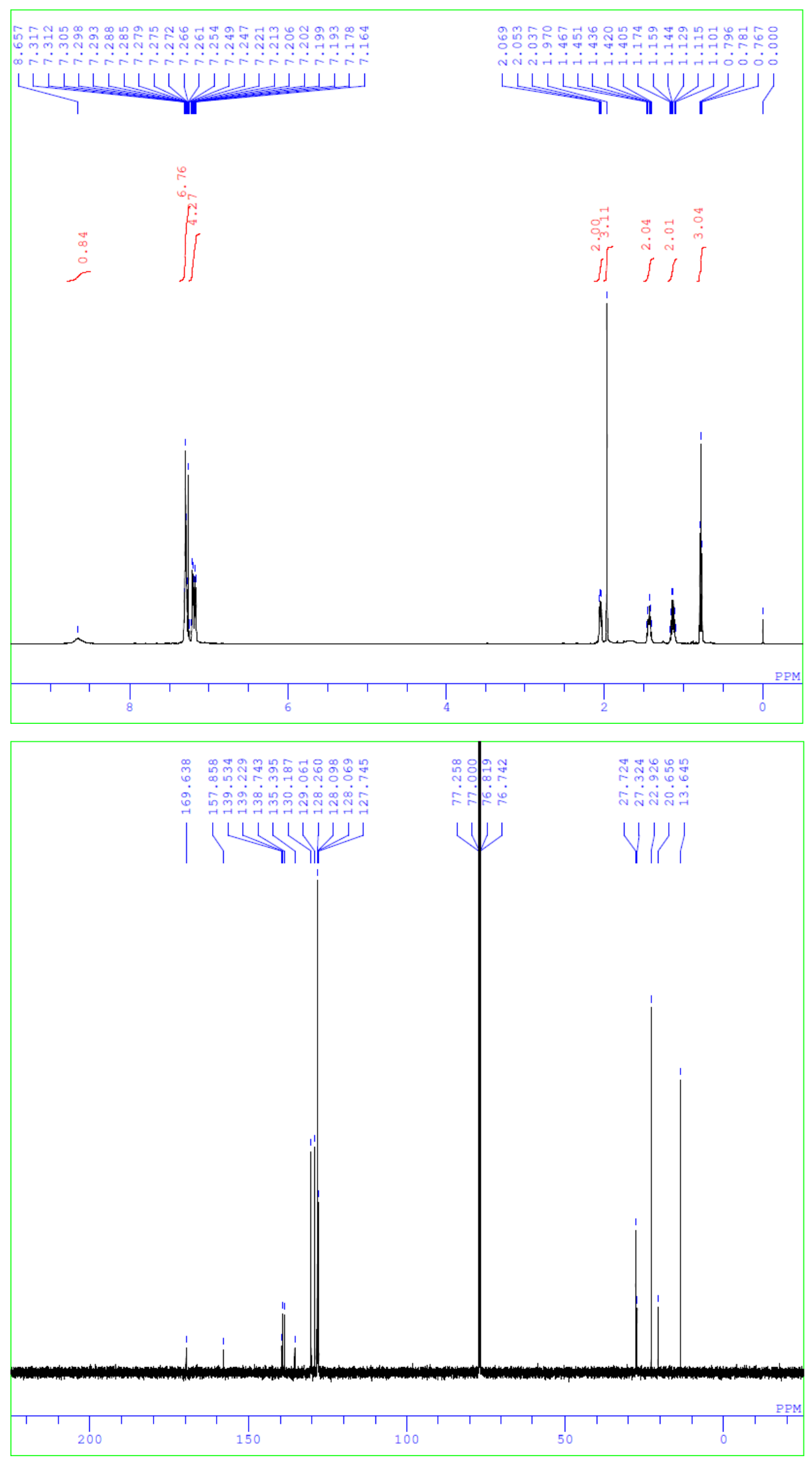

DFILE _ky_AcO oxime _carbon-1-1.als

DATIM 2014-10-29 22:53:40

OBNUC $13 \mathrm{C}$

EXMOD carbon.jxp

OBFRQ $\quad 125.77 \mathrm{MHz}$

OBSET $\quad 7.87 \mathrm{KHz}$

OBFIN $4.21 \mathrm{~Hz}$

POINT 26214

FREQU $31446.54 \mathrm{~Hz}$

SCANS 2830

ACQTM $\quad 0.8336 \mathrm{sec}$

$2.0000 \mathrm{sec}$

PW1 $2.0000 \mathrm{sec}$

IRNUC 1H

CTEMP 1 1 16.3 C

SLVNT CDCL 3

EXREF $77.00 \mathrm{pPm}$

$\mathrm{BF}$

$\begin{array}{lr}\text { BF } & 0.10 \mathrm{H} \\ \text { RGAIN } & 60\end{array}$ 
<smiles>N=C(C(=O)c1ccc2ccccc2c1)C(=C(c1ccccc1)c1ccccc1)c1ccccc1</smiles>

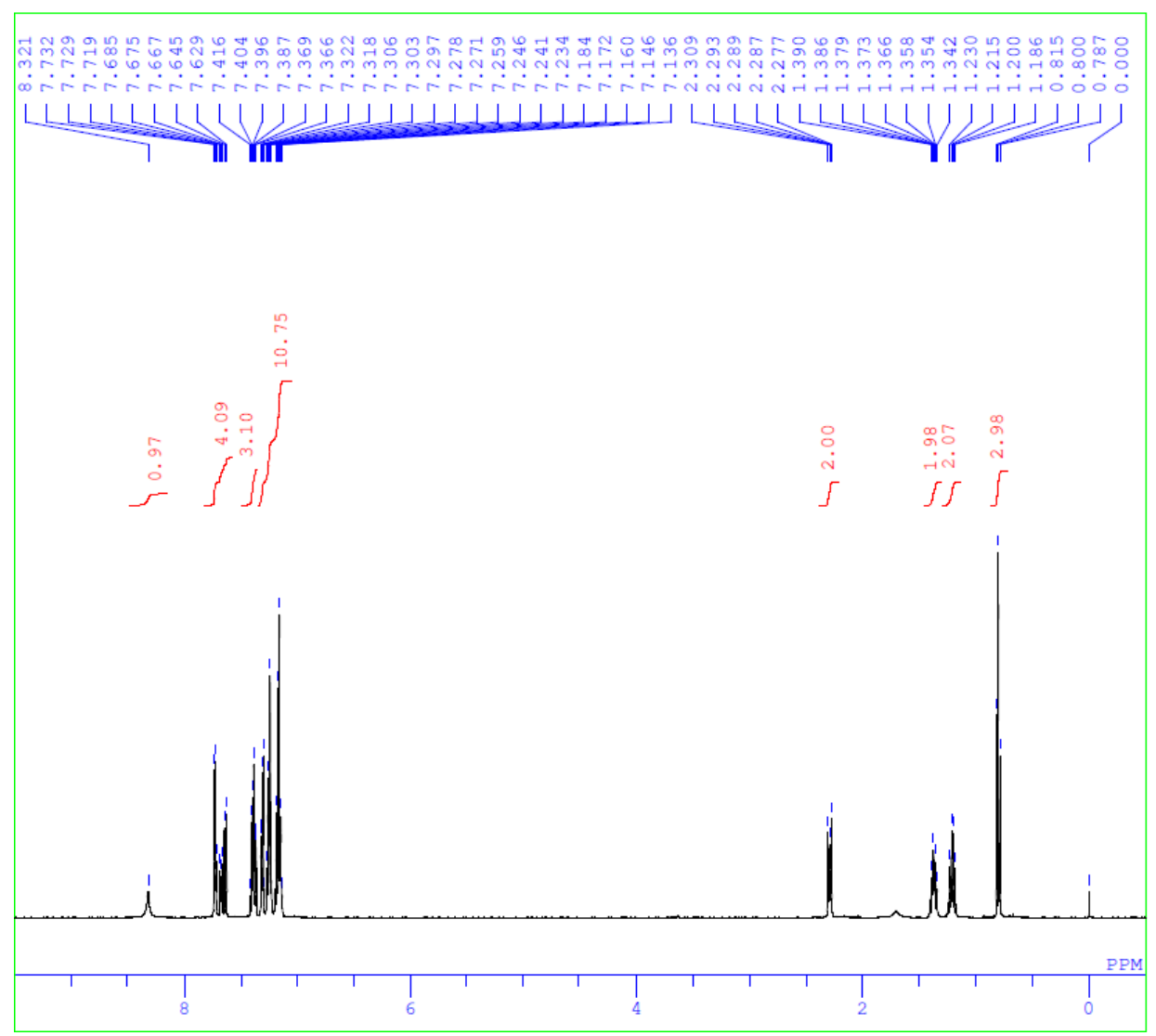

DFILE _ky_naphthalenthiol oxime_s1

COMNT ky_naphthalenthiol oxime_si

DATIM 2014-09-21 11:55:36

OBNUC 1

EXMOD proton. jxp $500.16 \mathrm{MHz}$

OBFRQ $\quad 500.16 \mathrm{MHz}$

$\begin{array}{ll}\text { OBSET } & 2.41 \mathrm{KHz} \\ \text { OBFIN } & 6.01 \mathrm{~Hz}\end{array}$

POINT

FREQU $\quad 7507.51 \mathrm{~Hz}$

$\begin{array}{lr}\text { SCANS } & 8 \\ \text { ACQTM } & 1.7459 \mathrm{sec}\end{array}$

PD

IRNUC $1 \mathrm{H}$

CTEMP $17.7 \mathrm{C}$

SLVNT CDCL3 30.00 pprn

BF $0.10 \mathrm{ppr}$

RGAIN $\quad 34$
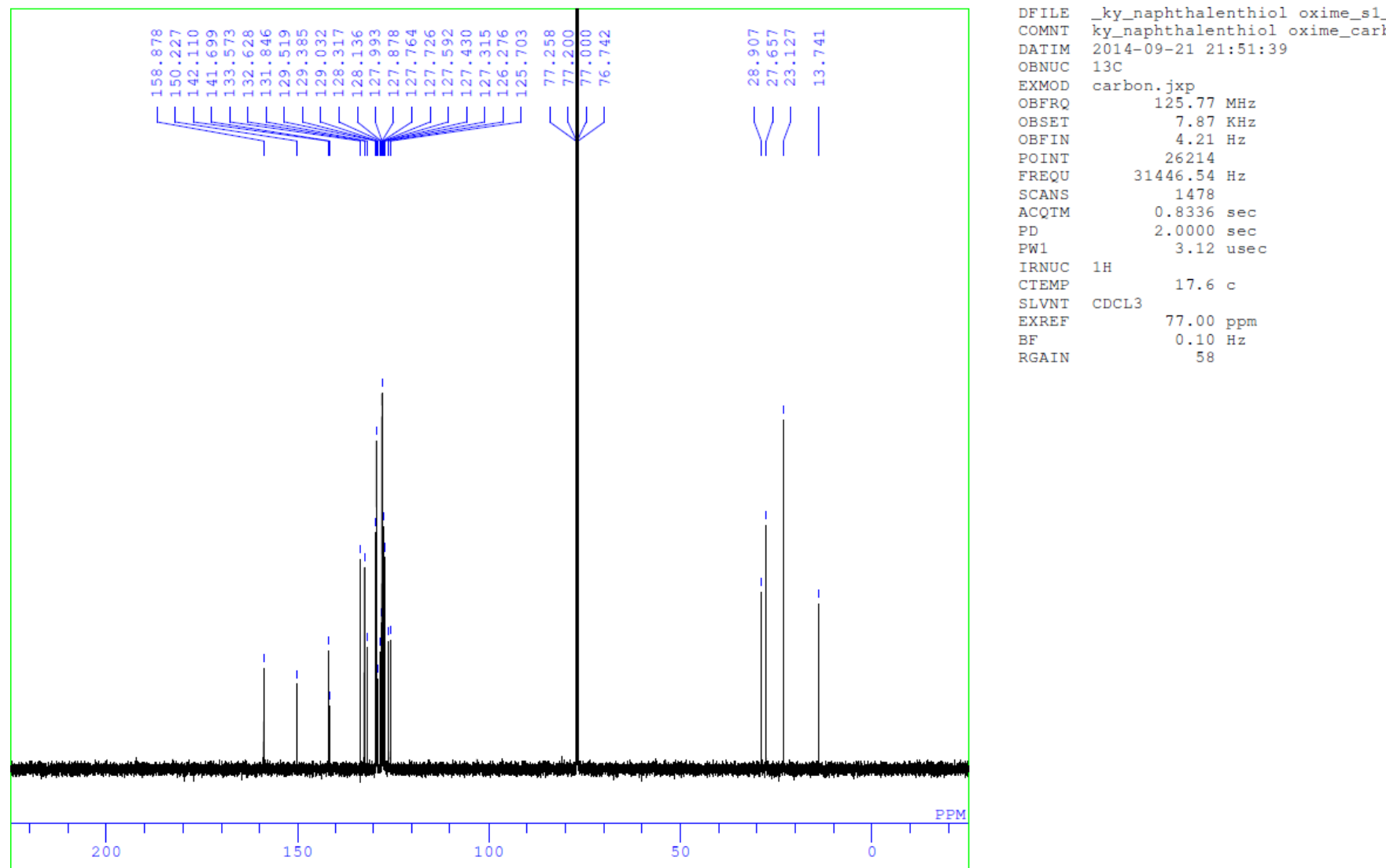
<smiles>N#C/C(C(=O)O)=C(\Br)c1ccccc1</smiles>

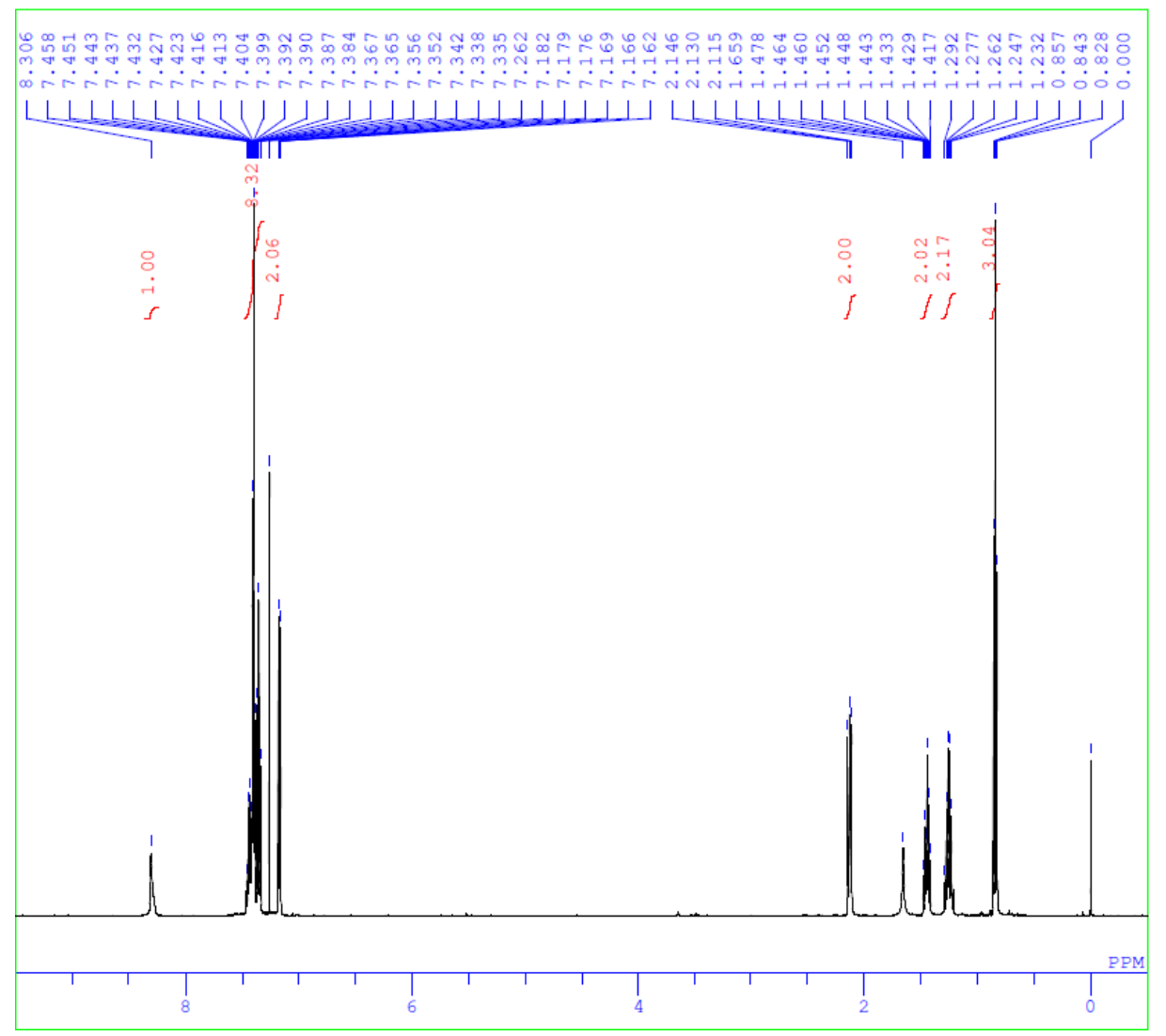

DFILE_ky_CN oxime-1-1.al

COMNT ky_CN oxime

DATIM 2014-09-15 22:26:38

OBNUC $1 \mathrm{H}$

EXMOD proton. jxp

OBFRQ $\quad 500.16 \mathrm{MHz}$

$\begin{array}{ll}\text { OBSET } & 2.41 \mathrm{KHz}\end{array}$

POINT 13107

FREQU $\quad 7507.51 \mathrm{~Hz}$

SCANS 8

ACQTM $1.7459 \mathrm{sec}$

PD
PW1

IRNUC $1 \mathrm{H}$

CTEMP

SLVNT

6.22 usec

EXR

RGAIN

$18.6 \mathrm{c}$

$\begin{array}{ll}\text { CDCL3 } & 0.00 \mathrm{ppm}\end{array}$

$0.10 \mathrm{~Hz}$

\section{RGA}

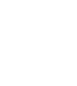

\section{年}
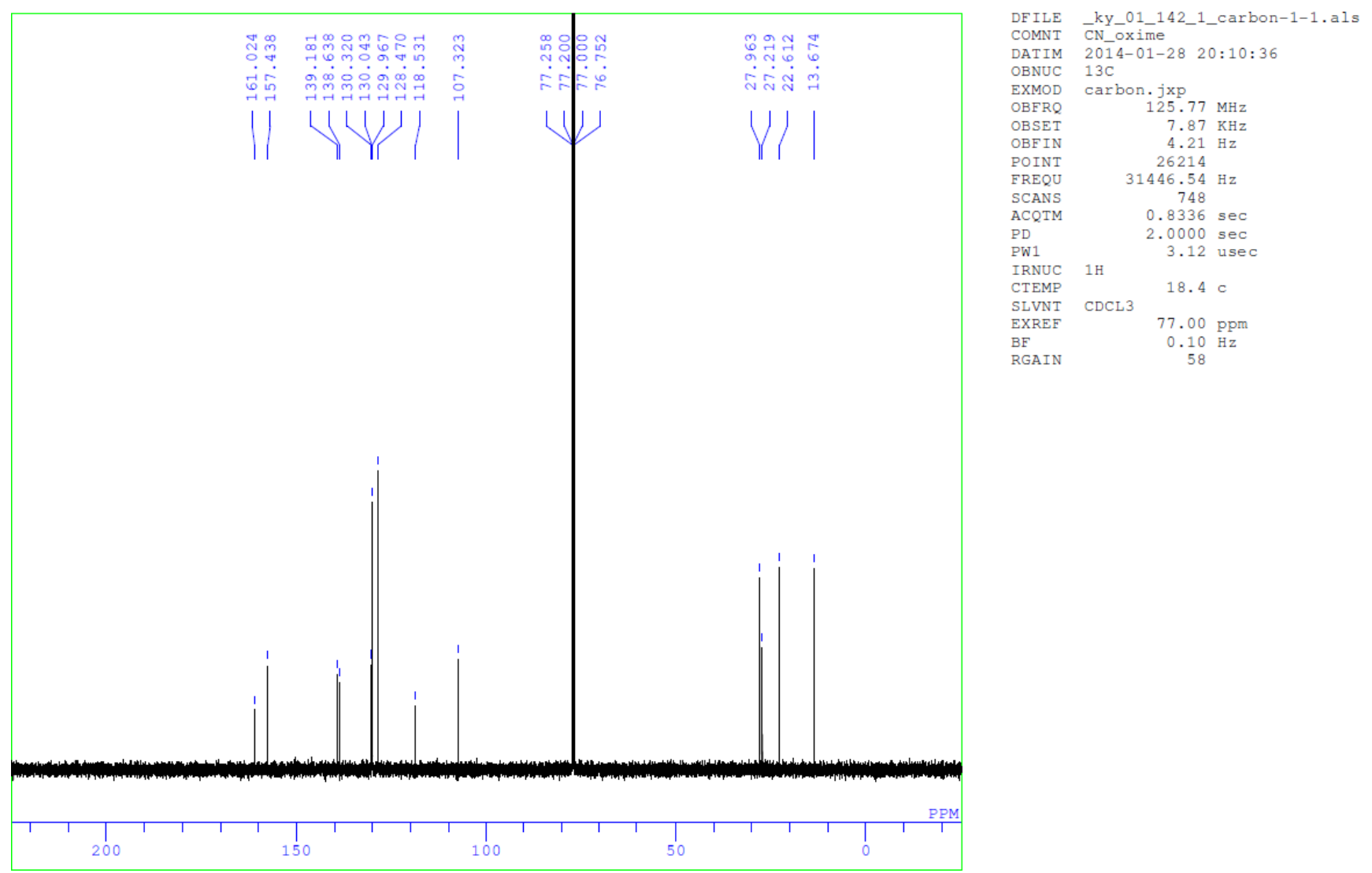

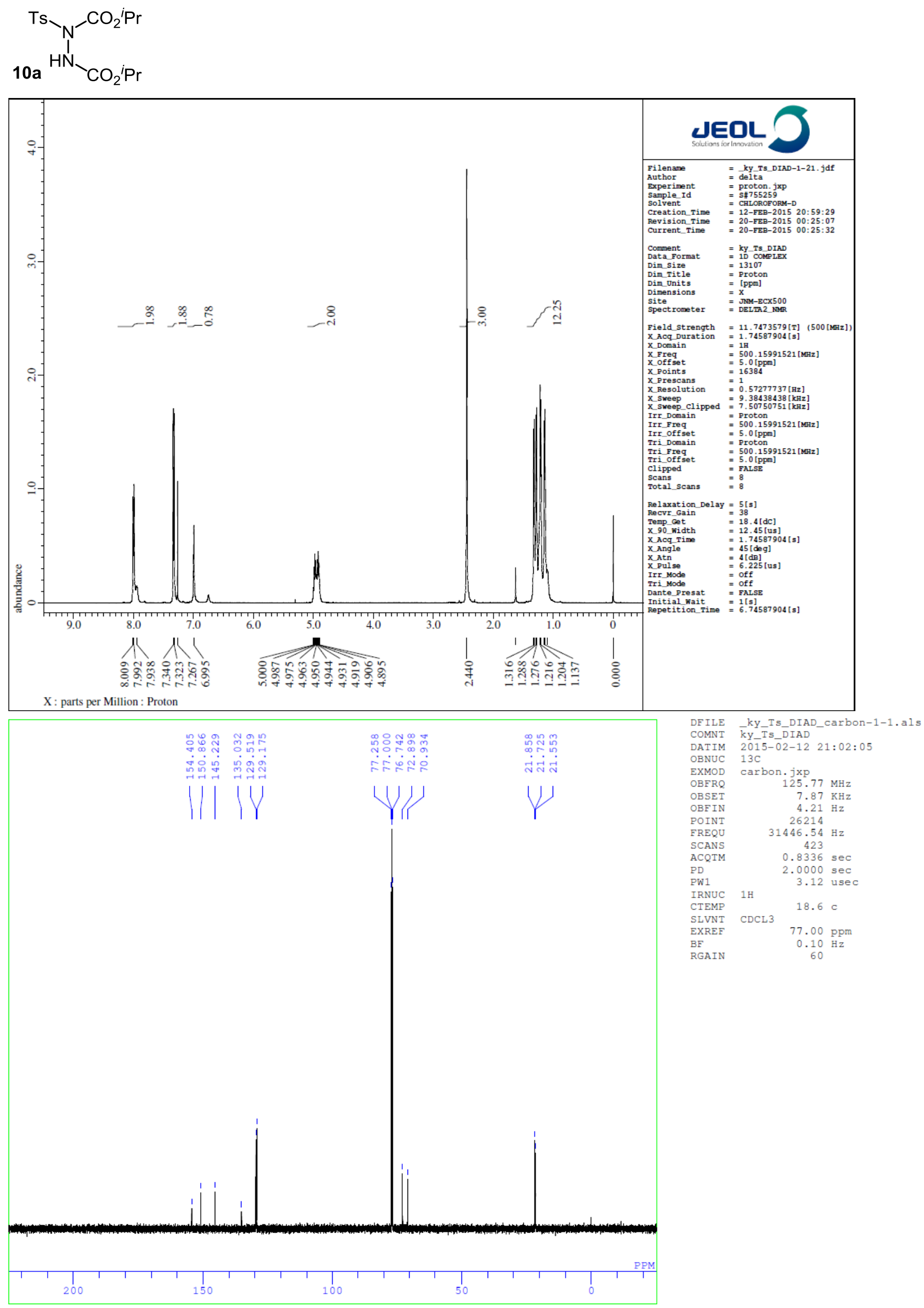


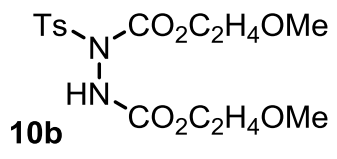

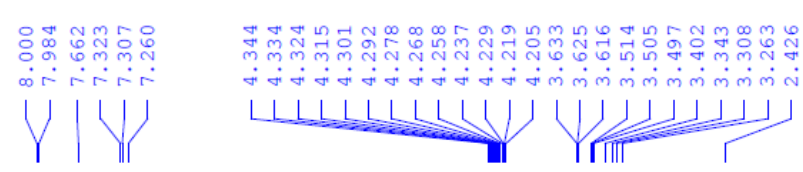

DFILE_TS_TSDMEAD_H-1-1.als

COMNT TSDMEAD

DATIM 2015-09-17 20:22:48

OBNUC $1 \mathrm{H}$

EXMOD proton.jxp

OBFRQ $\quad 500.16 \mathrm{MHz}$

OBSET $\quad 2.41 \mathrm{KHz}$

OBEIN $6.01 \mathrm{~Hz}$

$\begin{array}{lr}\text { POINT } & 13107\end{array}$

FREQU $\quad 7507.51 \mathrm{~Hz}$

8
SCANS

5.0000 sec

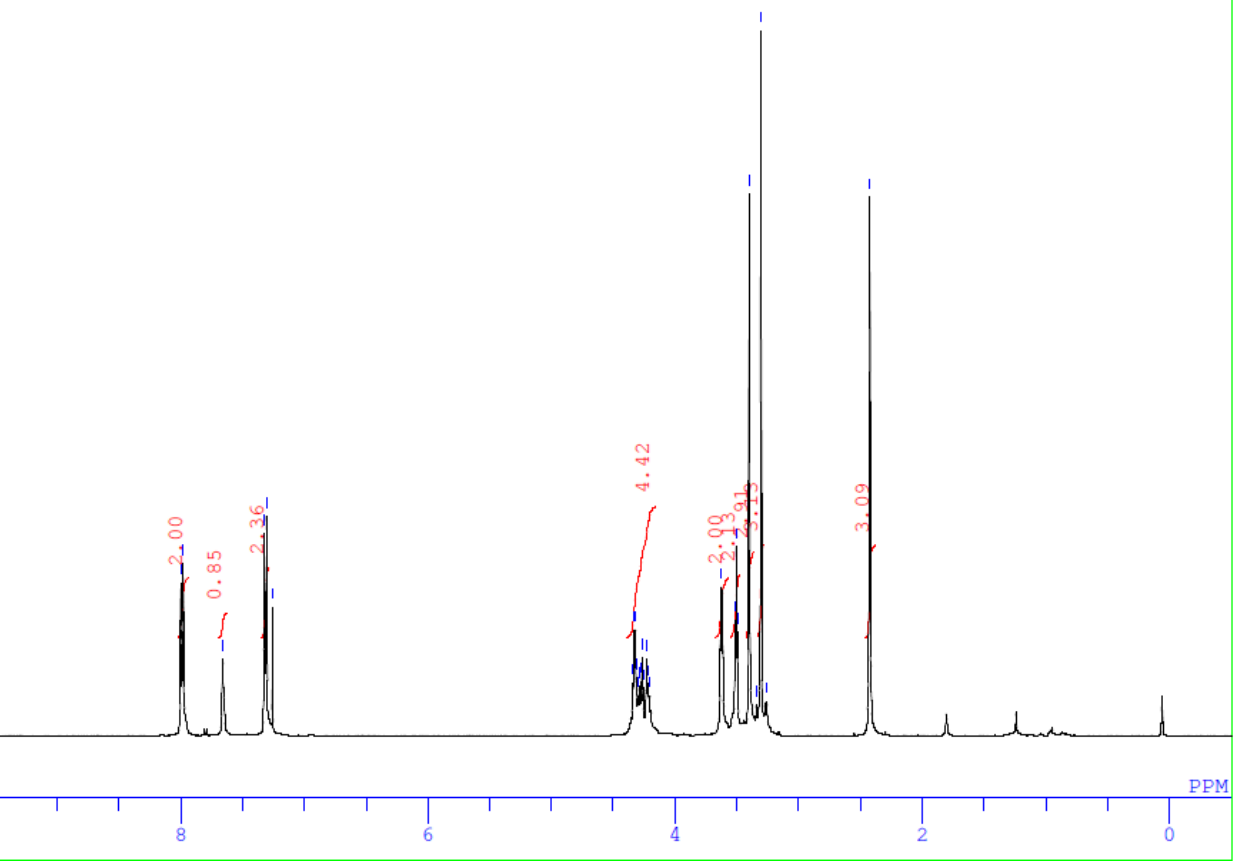

PW

6.22 usec

IRNUC $1 \mathrm{H}$

SLVNT CDCL3

EXREF

$\mathrm{BF}$

RGAIN

$18.6 \mathrm{c}$

7. $26 \mathrm{ppm}$

\begin{tabular}{|c|c|c|}
\hline DFILE & TS_TsDMEAD_C & C-2-1.al \\
\hline COMNT & TSDMEAD & \\
\hline DATIM & $2015-09-1720$ & $0: 24: 08$ \\
\hline OBNUC & $13 \mathrm{C}$ & \\
\hline EXMOD & carbon.jxp & \\
\hline OBFRQ & 125.77 & $\mathrm{MHz}$ \\
\hline OBSET & 7.87 & $\mathrm{KHz}$ \\
\hline OBFIN & 4.21 & $\mathrm{~Hz}$ \\
\hline POINT & 26214 & \\
\hline$F R E Q U$ & 31446.54 & $\mathrm{~Hz}$ \\
\hline SCANS & 257 & \\
\hline ACQTM & 0.8336 & sec \\
\hline PD & 2.0000 & $\sec$ \\
\hline PW1 & 3.12 & usec \\
\hline IRNUC & $1 \mathrm{H}$ & \\
\hline CTEMP & 19.0 & $\mathrm{c}$ \\
\hline SLVNT & CDCL 3 & \\
\hline EXREF & 77.00 & ppm \\
\hline $\mathrm{BF}$ & 0.10 & $\mathrm{~Hz}$ \\
\hline RGAIN & 60 & \\
\hline
\end{tabular}

$0.10 \mathrm{~Hz}$$$
34
$$

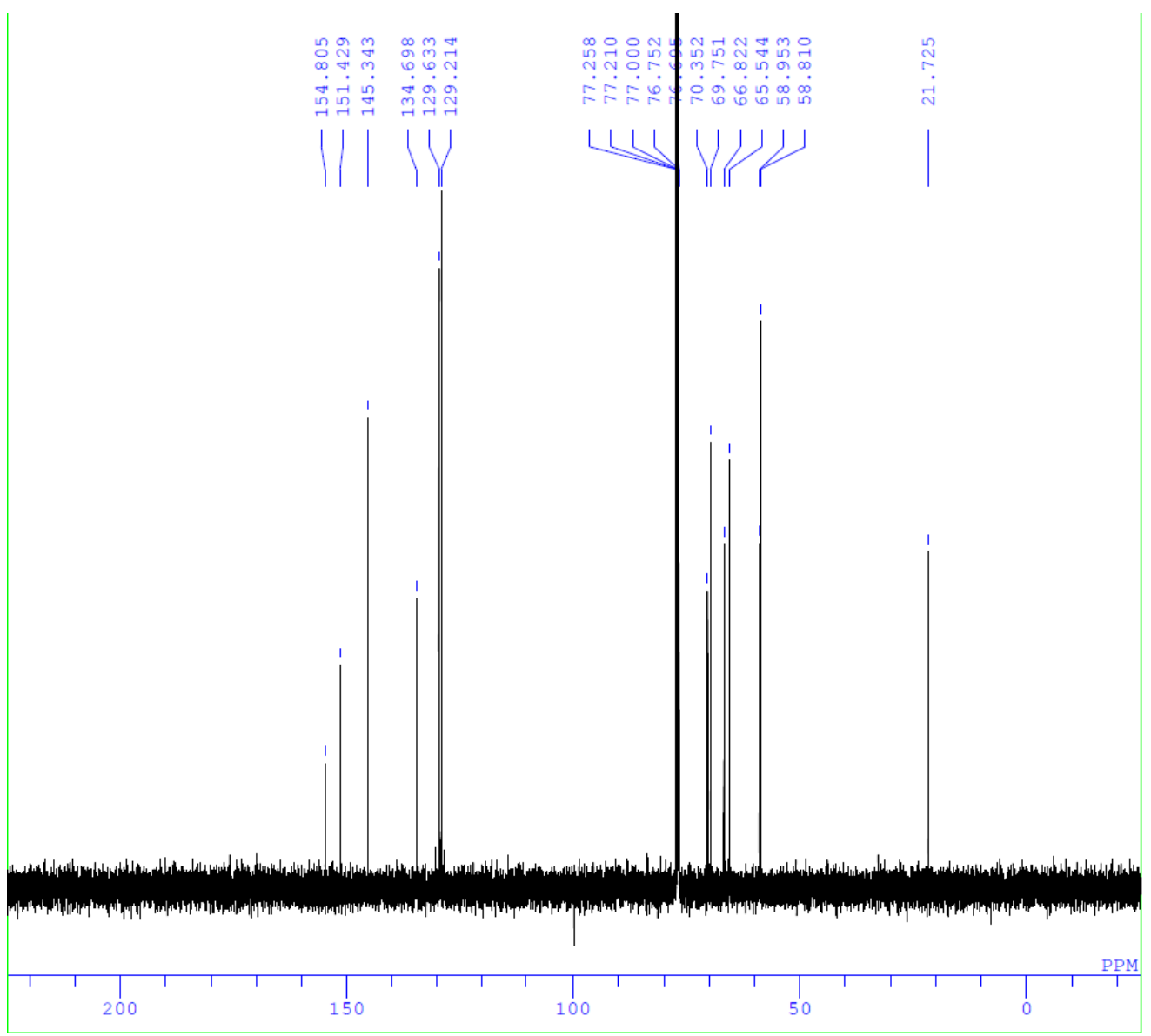


ORTEP Figure of 2-tosylenoxime bromobenzoate 5a' (CCDC1056831) (with thermal elipsoids at $50 \%$ probability)

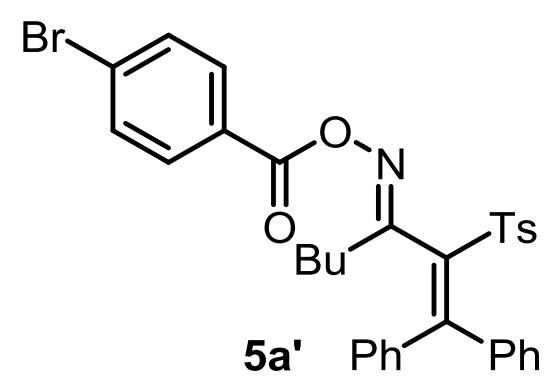

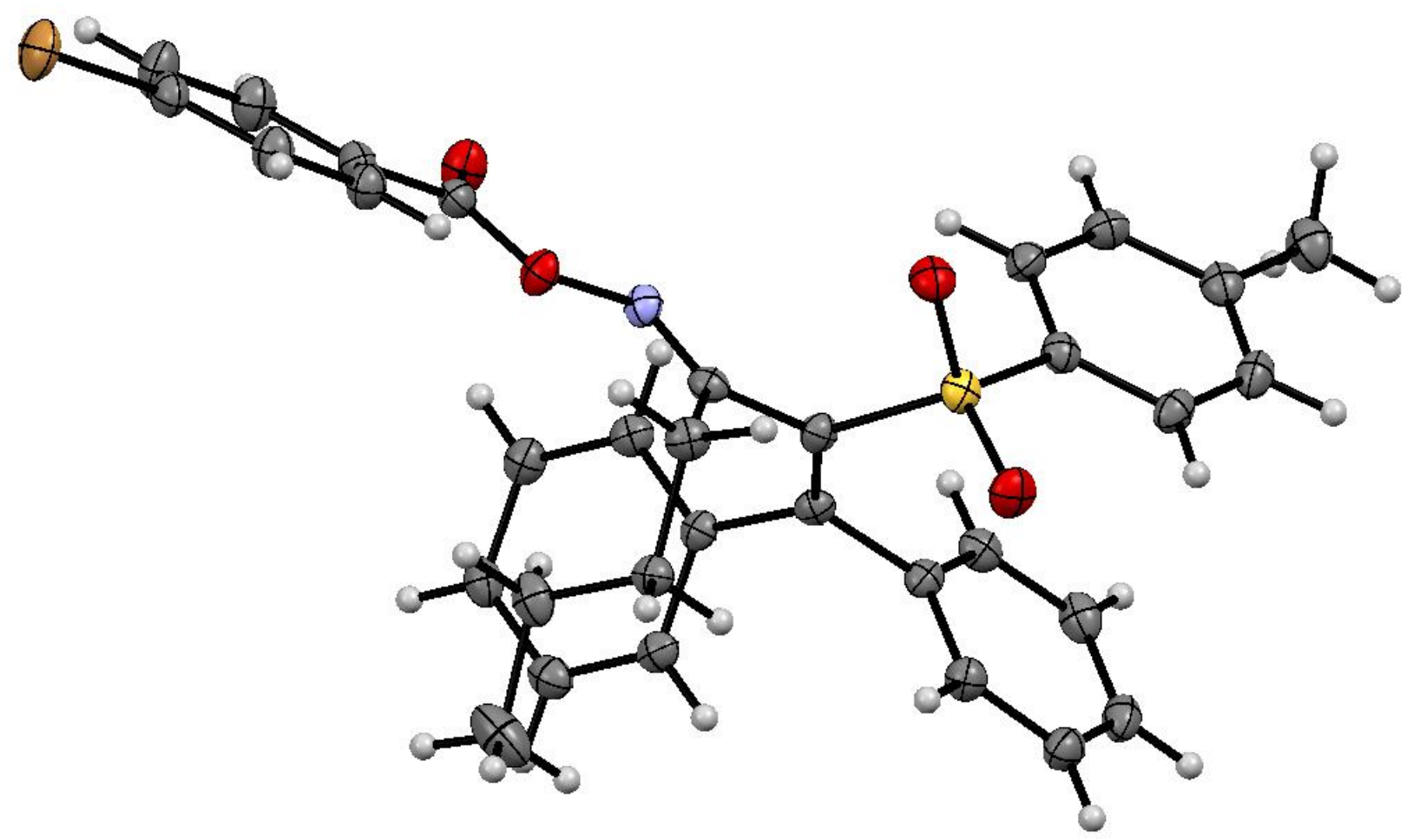


Crystal data and structure refinement for 2-tosylenoxime bromobenzoate 5a' (CCDC1056831)

Empirical formula

Formula weight

Temperature

Wavelength

Crystal system

Space group

Unit cell dimensions

Volume

$\mathrm{Z}$

Density (calculated)

Absorption coefficient

F(000)

Crystal size

Theta range for data collection

Index ranges

Reflection collected

Independent reflections

Completeness to theta $=27.48^{\circ}$

Max. min. transmission

Refinement method

Data / restraints / parameters

Goodness-of-fit on $F^{2}$

Final $\mathrm{R}$ indices [ $>2 \operatorname{sigma}(I)]$

$\mathrm{R}$ indices (all data)

Largest diff. peak and hole
$\mathrm{C}_{33} \mathrm{H}_{30} \mathrm{BrNO}_{4} \mathrm{~S}$

616.57

$123 \mathrm{~K}$

$0.71075 \AA$

triclinic

P-1 (\#2)

$\mathrm{a}=7.6584(2) \AA$

$\alpha=88.9061(7)^{\circ}$

$\mathrm{b}=7.9059(2) \AA$

$\beta=82.0333(7)^{\circ}$

$\mathrm{c}=24.3938(7) \AA$

$\gamma=79.5504(7)^{\circ}$
2

$1.423 \mathrm{~g} / \mathrm{cm}^{3}$

1.545

636.00

$0.180 \times 0.150 \times 0.020 \mathrm{~mm}$

3.00 to 27.48

$-9<=\mathrm{h}<=9,-10<=\mathrm{k}<=10,-31<=\mathrm{l}<=3$

14402

$6556\left(\mathrm{R}_{\mathrm{int}}=0.0239\right)$

$99.6 \%$

0.784 and 0.970

Full-matrix least-squares on $\mathrm{F}^{2}$

$6556 / 0 / 363$

1.114

$\mathrm{R}_{1}=0.0373$

$\mathrm{R}_{1}=0.0470, \mathrm{wR}_{2}=0.1071$

1.24 and $-0.45 \mathrm{e} \cdot \AA^{-3}$ 
ORTEP Figure of N-tosylisozasoline 6 (CCDC1056830)

with thermal elipsoids at $50 \%$ probability
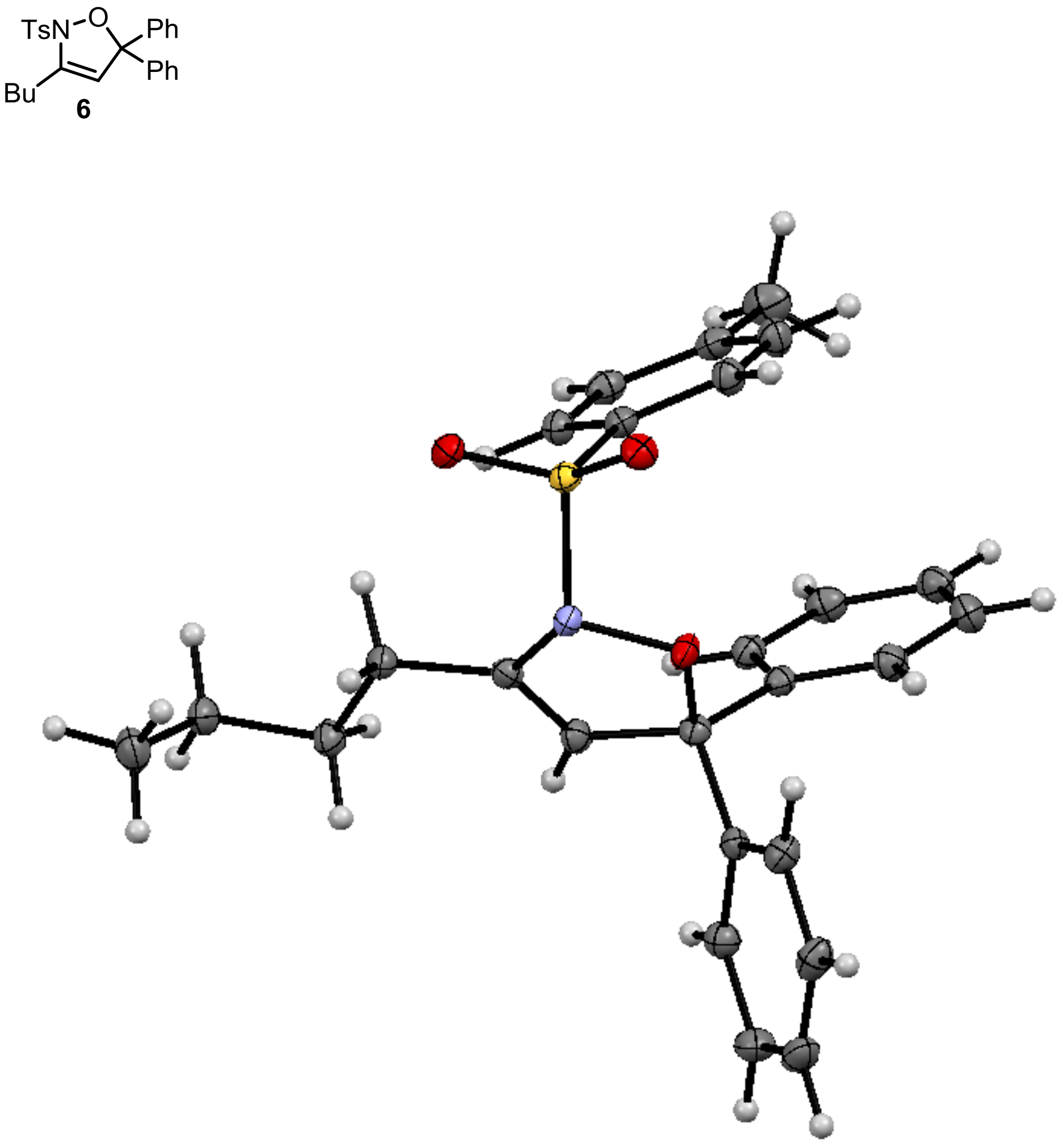
Crystal data and structure refinement for N-tosylisozasoline 6 (CCDC1056830)

Empirical formula

Formula weight

Temperature

Wavelength

Crystal system

Space group

Unit cell dimensions

Volume

$\mathrm{Z}$

Density (calculated)

Absorption coefficient

$F(000)$

Crystal size

Theta range for data collection

Index ranges

Reflection collected

Independent reflections

Completeness to theta $=27.47^{\circ}$

Max. min. transmission

Refinement method

Data / restraints / parameters

Goodness-of-fit on $F^{2}$

Final $\mathrm{R}$ indices [ $\$ 2 \operatorname{sigma}(I)]$

$\mathrm{R}$ indices (all data)

Largest diff. peak and hole
$\mathrm{C}_{26} \mathrm{H}_{27} \mathrm{NO}_{3} \mathrm{~S}$

433.56

$123 \mathrm{~K}$

$0.71075 \AA$

monoclinic

P2 1 /n (\#14)

$\mathrm{a}=12.3930(3) \AA$

$\mathrm{b}=11.2794(2) \AA$

$\beta=100.1640(7)^{\circ}$

$\mathrm{c}=15.7786(3) \AA$

$\mathrm{V}=2171.00(7) \AA^{3}$

4

$1.326 \mathrm{~g} / \mathrm{cm}^{3}$

0.178

920.00

$0.180 \times 0.170 \times 0.170 \mathrm{~mm}$

3.19 to $27.48^{\circ}$

$-16<=\mathrm{h}<=16,-14<=\mathrm{k}<=14,-20<=\mathrm{l}<=18$

21074

$4967\left(\mathrm{R}_{\mathrm{int}}=0.0225\right)$

$99.8 \%$

0.871 and 0.970

Full-matrix least-squares on $\mathrm{F}^{2}$

4967/0/282

1.132

$\mathrm{R}_{1}=0.0439$

$\mathrm{R}_{1}=0.0460, \mathrm{wR}_{2}=0.1130$

0.76 and -0.35 e. $\AA^{-3}$ 
ORTEP Figure of (Z)-o-tolylenoxime (Z)-5p (CCDC1056832)

with thermal elipsoids at $50 \%$ probability

(Z)-5p<smiles>Cc1ccccc1C(=C([As])C(Cc1ccccc1)=NO)c1ccccc1</smiles>

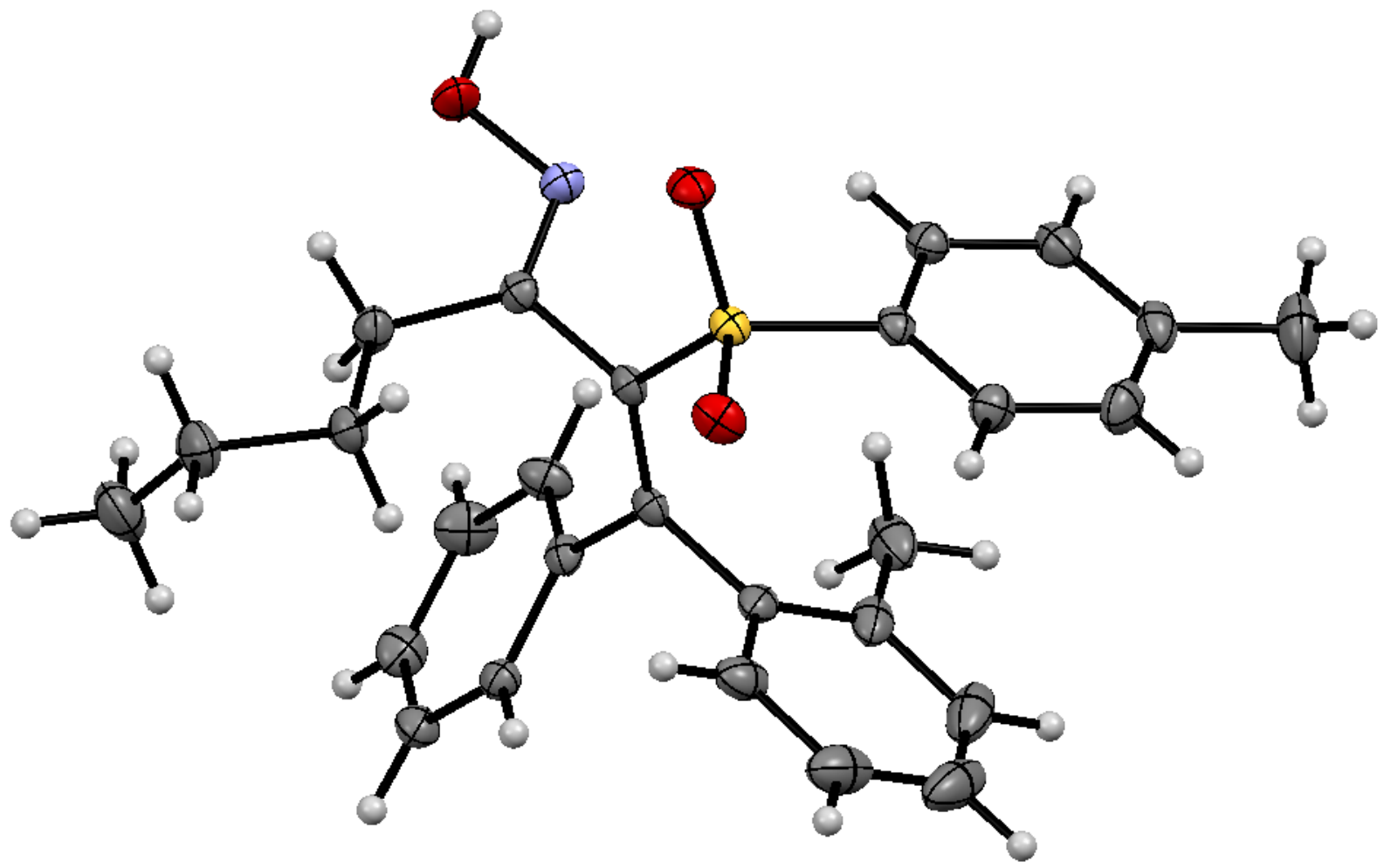


Crystal data and structure refinement for (Z)-o-tolylenoxime (Z)-5p (CCDC1056832)

Empirical formula

Formula weight

Temperature

Wavelength

Crystal system

Space group

Unit cell dimensions

Volume

$\mathrm{Z}$

Density (calculated)

Absorption coefficient

F(000)

Crystal size

Theta range for data collection

Index ranges

Reflection collected

Independent reflections

Completeness to theta $=27.484^{\circ}$

Max. min. transmission

Refinement method

Data / restraints / parameters

Goodness-of-fit on $F^{2}$

Final $\mathrm{R}$ indices $[\triangle 2 \operatorname{sigma}(I)]$

$\mathrm{R}$ indices (all data)

Largest diff. peak and hole
$\mathrm{C}_{27} \mathrm{H}_{29} \mathrm{NO}_{3} \mathrm{~S}$

447.59

$123 \mathrm{~K}$

$0.71075 \AA$

monoclinic

C2/c (\#15)

$\mathrm{a}=19.0086(4) \AA$

$\mathrm{b}=8.50881(15) \AA \quad \beta=103.9455(7)^{\circ}$

$\mathrm{c}=30.3715(6) \AA$

$\mathrm{V}=4767.50(15) \AA^{3}$

8

$1.247 \mathrm{~g} / \mathrm{cm}^{3}$

0.164

1904.00

$0.170 \times 0.160 \times 0.150 \mathrm{~mm}$

3.10 to 27.50

$-24<=\mathrm{h}<=24,-11<=\mathrm{k}<=11,-39<=\mathrm{l}<=39$

40046

$5465\left(\mathrm{R}_{\text {int }}=0.0220\right)$

$99.8 \%$

0.909 and 0.976

Full-matrix least-squares on $\mathrm{F}^{2}$

$5465 / 0 / 293$

1.053

$\mathrm{R}_{1}=0.0378, \mathrm{wR}_{2}=0.0954$

$\mathrm{R}_{1}=0.0412, \mathrm{wR}_{2}=0.0972$

0.42 and $-0.34 \mathrm{e} \cdot \AA^{-3}$ 
ORTEP Figure of 2-nitroenoxime 9i (CCDC1056829)

with thermal elipsoids at $50 \%$ probability<smiles>CC(C)(Br)C(=C(C(=NO)[N+](=O)[O-])c1ccccc1)c1ccccc1</smiles>

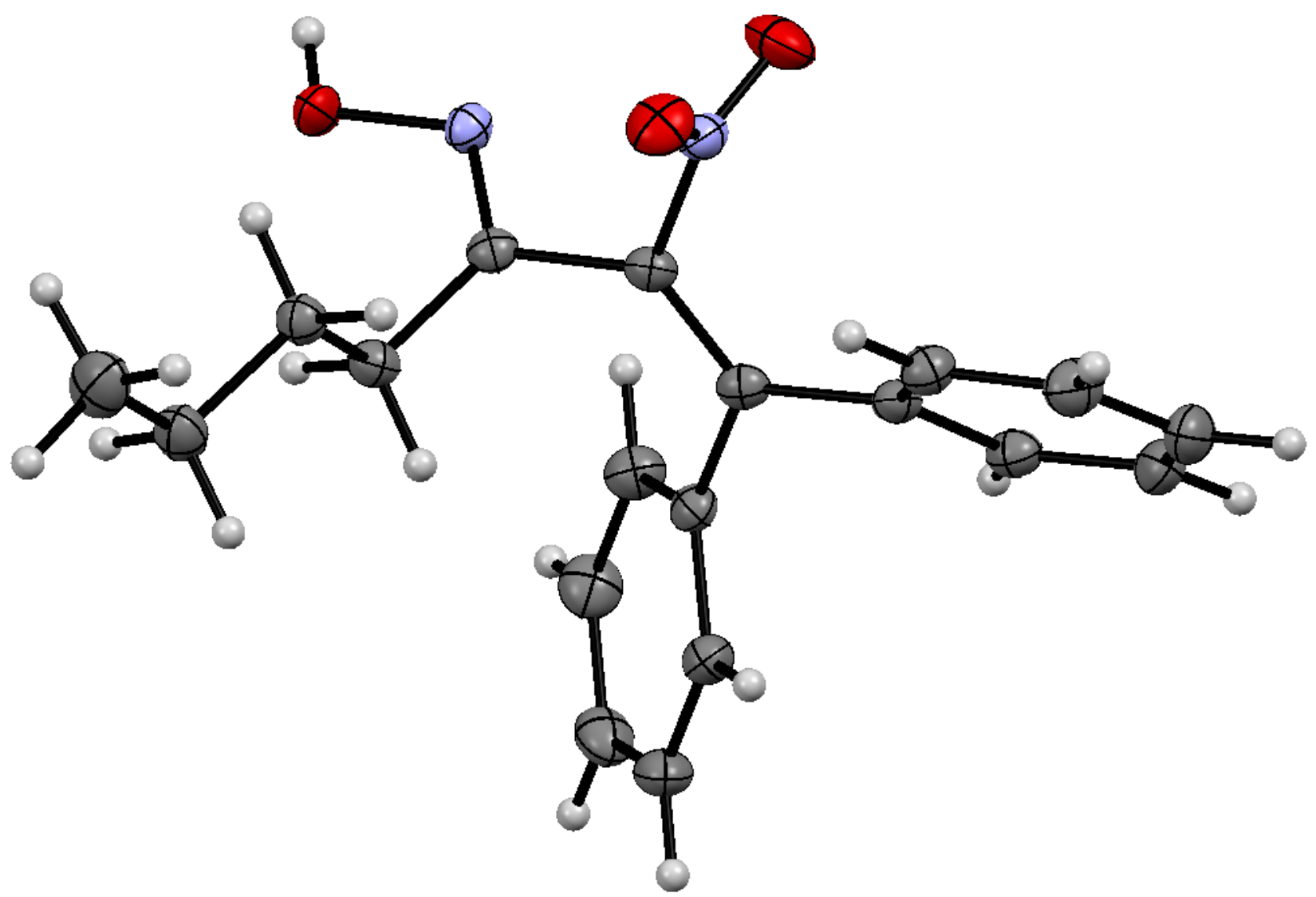


Crystal data and structure refinement for 2-nitroenoxime 9i (CCDC1056829)

Empirical formula

Formula weight

Temperature

Wavelength

Crystal system

Space group

Unit cell dimensions

Volume

Z

Density (calculated)

Absorption coefficient

F(000)

Crystal size

Theta range for data collection

Index ranges

Reflection collected

Independent reflections

Completeness to theta $=27.48^{\circ}$

Max. min. transmission

Refinement method

Data / restraints / parameters

Goodness-of-fit on $F^{2}$

Final $\mathrm{R}$ indices $[\triangle 2 \operatorname{sigma}(I)]$

$\mathrm{R}$ indices (all data)

Largest diff. peak and hole
$\mathrm{C}_{19} \mathrm{H}_{20} \mathrm{~N}_{2} \mathrm{O}_{3}$

324.38

$113 \mathrm{~K}$

$0.71075 \AA$

triclinic

P-1 (\#2)

$a=8.6333(3) \AA \quad \alpha=96.9079(7)^{\circ}$

$\mathrm{b}=8.8406(3) \AA \quad \beta=105.0263(8)^{\circ}$

$c=12.4265(4) \AA \gamma=106.9852(8) \circ$

$\mathrm{V}=856.09(4) \AA^{3}$

2

$1.258 \mathrm{~g} / \mathrm{cm}^{3}$

$0.086 \mathrm{~mm}^{-1}$

344.00

$0.190 \times 0.180 \times 0.060 \mathrm{~mm}$

3.25 to $27.50^{\circ}$

$-11<=\mathrm{h}<=11,-11<=\mathrm{k}<=11,-16<=\mathrm{l}<=14$

8614

$3913\left(\mathrm{R}_{\mathrm{int}}=0.0172\right)$

$99.6 \%$

0.897 and 0.995

Full-matrix least-squares on $\mathrm{F}^{2}$

$3913 / 0 / 219$

1.216

$\mathrm{R}_{1}=0.0513, \mathrm{wR}_{2}=0.1468$

$\mathrm{R}_{1}=0.0599, \mathrm{wR}_{2}=0.1468$

0.51 and $-0.30 \mathrm{e} \cdot \AA^{-3}$ 
ORTEP Figure of azirine 9j' (CCDC1056828)

with thermal elipsoids at $50 \%$ probability<smiles>O=C(Br)/C(=N\O)C1=NC1(c1ccccc1)c1ccccc1</smiles>

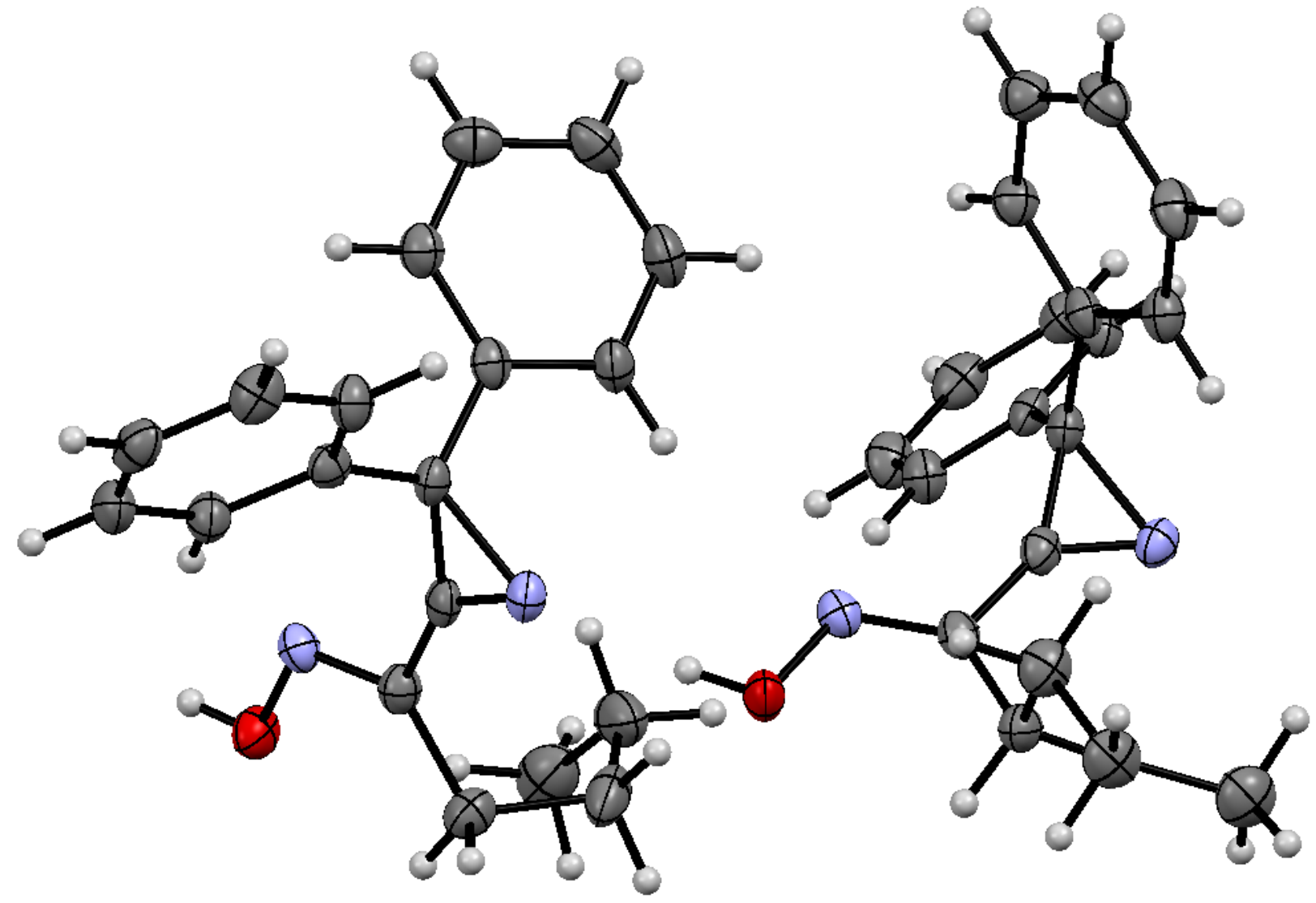


Empirical formula

Formula weight

Temperature

Wavelength

Crystal system

Space group

Unit cell dimensions

Volume

$\mathrm{Z}$

Density (calculated)

Absorption coefficient

$F(000)$

Crystal size

Theta range for data collection

Index ranges

Reflection collected

Independent reflections

Completeness to theta $=25.242$

Max. min. transmission

Refinement method

Data / restraints / parameters

Goodness-of-fit on $F^{2}$

Final $\mathrm{R}$ indices [ $>2 \operatorname{sigma}(I)]$

$\mathrm{R}$ indices (all data)

Lagest diff. peak and hole
$\mathrm{C}_{19} \mathrm{H}_{20} \mathrm{~N}_{2} \mathrm{O}$

292.38

$123 \mathrm{~K}$

$0.71075 \AA$

monoclinic

$\mathrm{P} 21$ (\#4)

$\mathrm{a}=12.2761(3) \AA$

$\mathrm{b}=7.87636(18) \AA$

$\beta=92.7067(8)^{\circ}$

$\mathrm{c}=16.8396(4) \AA$

$\mathrm{V}=1626.42(7) \AA^{3}$

4

$1.194 \mathrm{~g} / \mathrm{cm}^{3}$

$0.074 \mathrm{~mm}^{-1}$

624.00

$0.090 \times 0.060 \times 0.010 \mathrm{~mm}$

3.00 to $25.34^{\circ}$

$-14<=\mathrm{h}<=14,-9<=\mathrm{k}<=9,-20<=\mathrm{l}<=20$

23251

$5857\left(\mathrm{R}_{\text {int }}=0.0422\right)$

$99.8 \%$

0.794 and 0.999

Full-matrix least-squares on $\mathrm{F}^{2}$

$5857 / 1 / 401$

1.090

$\mathrm{R}_{1}=0.0397, \mathrm{wR}_{2}=0.0901$

$\mathrm{R}_{1}=0.0545, \mathrm{wR}_{2}=0.1008$

0.18 and $-0.22 \mathrm{e} \cdot \AA^{-3}$ 